\title{
Novel mechanisms of neuronal and hormonal regulation of large intestinal electrolyte transport
}

Andrew John Nickerson

West Virginia University, anicker2@mix.wvu.edu

Follow this and additional works at: https://researchrepository.wvu.edu/etd

Part of the Digestive, Oral, and Skin Physiology Commons, and the Medical Physiology Commons

\section{Recommended Citation}

Nickerson, Andrew John, "Novel mechanisms of neuronal and hormonal regulation of large intestinal electrolyte transport" (2021). Graduate Theses, Dissertations, and Problem Reports. 8298.

https://researchrepository.wvu.edu/etd/8298

This Dissertation is protected by copyright and/or related rights. It has been brought to you by the The Research Repository @ WVU with permission from the rights-holder(s). You are free to use this Dissertation in any way that is permitted by the copyright and related rights legislation that applies to your use. For other uses you must obtain permission from the rights-holder(s) directly, unless additional rights are indicated by a Creative Commons license in the record and/ or on the work itself. This Dissertation has been accepted for inclusion in WVU Graduate Theses, Dissertations, and Problem Reports collection by an authorized administrator of The Research Repository @ WVU.

For more information, please contact researchrepository@mail.wvu.edu. 


\title{
Novel mechanisms of neuronal and hormonal regulation of large intestinal electrolyte transport
}

\author{
Andrew J. Nickerson \\ Dissertation submitted to the College of Medicine at West Virginia University \\ In partial fulfillment of the requirements for the degree of Doctor of Philosophy in Cellular and \\ Integrative Physiology \\ Vazhaikkurichi Rajendran, Ph.D., Mentor \\ Stanley Hileman, Ph.D., Chair \\ Hang Gang Yu, Ph.D. \\ Michael Schaller, Ph.D. \\ Eric Kelley, Ph.D. \\ Department of Physiology Pharmacology and Neuroscience \\ Department of Biochemistry \\ Morgantown, WV 2021
}

KEY WORDS: colon, ion transport, IBD, IBS-D, Ussing chamber

Copyright 2021: Andrew Nickerson 


\section{ABSTRACT: \\ Novel mechanisms of neuronal and hormonal regulation of large intestinal electrolyte transport}

\section{Andrew J. Nickerson}

The mammalian colon plays a vital role in maintaining overall electrolyte and fluid homeostasis in the body, and it possesses the ability to either secrete or absorb water depending upon the needs of the organism. Secretion or absorption of electrolyte ions, such as $\mathrm{Na}^{+}, \mathrm{Cl}^{-}$and $\mathrm{K}^{+}$, drives the movement of water across the colonic epithelium - the inner gut lining where all transport takes place. Aberrant absorptive and/or secretory activity within the colonic epithelium is central to the pathogenesis of many gastrointestinal diseases, such as irritable bowel syndrome (IBS), inflammatory bowel disease (IBD), constipation and infectious diarrhea. As such, therapeutic control of colonic electrolyte transport is critical to the management of these conditions. Two of its most important regulators - the enteric nervous system (ENS) and the corticosteroid hormones - provide key avenues by which colonic epithelial transport activity can be modulated or corrected, in the case of pharmacotherapeutic intervention. The work described in this dissertation characterizes two separate, novel aspects of regulatory control that may be the targets of future therapeutic drug development. First is the discovery and characterization of voltage gated $\mathrm{K}^{+}\left(\mathrm{K}_{\mathrm{V}} 7\right)$ channels in the ENS as governors of ENS activity and resultant epithelial transport function. Second is the study of the epithelial $\mathrm{N}^{+}, \mathrm{K}^{+}, 2 \mathrm{Cl}^{-}(\mathrm{NKCC1})$ co-transporter and its regulation by the corticosteroid hormone, aldosterone, in controlling epithelial $\mathrm{K}^{+}$and $\mathrm{Cl}^{-}$ transport. The findings from this work set the stage for development of targeted therapies aimed at these two mechanisms, which may lead to improved management of intestinal pathologies such as IBS, IBD or other diarrheal conditions. 


\section{ACKNOWLEDGEMENTS:}

I would first like to thank my parents for encouraging me to challenge myself in my academic affairs, starting in grade school and continuing through my college and graduate education. To my dad, thank you for instilling in me an interest in science, a general curiosity for exploring how things work, and a belief that learning is one of the most worthwhile activities available to us. To my mom, thank you for all of your help in navigating my path through school and for giving me so much guidance along the way. You set the best possible example for how to be a valuable and supportive member of any team, community or family. On top of this, I can merely speculate as to how many deadlines were met only because you reminded me of them. To my wife, Rachel, thank you so much for your endless support and for inspiring me to be the best version of myself. You were my biggest advocate as my girlfriend when I started this program, and you maintain this distinction now, as my wife. You have made our home truly feel like a home, which ultimately makes all other achievements, including this one, possible. To my siblings, thank you for not only tolerating me on my visits back to Wheeling, but making them so wonderful just by being there to hang out together (all in the same room) like when we were growing up. To my parents-in-law, thank you for all you've done to help me and Rachel, and for taking me in to your wonderful family. To my mentor, Raj, thank you for pushing me to pursue my own interests, solve problems independently, and in doing so, allowing me to grow into a successful scientific investigator. To my committee members, thank you for your guidance (and patience!) in helping me to see this project through to completion. To my former lab mates, Drew, Trey, Emily and Karthi, thank you for making my time in lab more enjoyable, and for talking through ideas, both good and bad. Thanks to Trey, especially, for helping to develop the groundwork which has made much of my dissertation work possible. To Mandy, thanks for all of your assistance and patience 
throughout the many hours I have spent in the imaging facility. To my all of my other family and friends, thank you for being supportive of me and for helping to maintain focus on what the most important things in life really are - among them, spending time with those that you love. Lastly, I want to thank Dr. Greg Dick for first introducing me to how rewarding the discipline of biomedical research can be. You have changed the course of my life as much as anyone, and I am forever indebted to you for inspiring an interest in physiology, as well as setting an example of true mentorship. To all those mentioned above, your support has made my success possible. I am grateful to each and every one of you. 


\section{TABLE OF CONTENTS:}

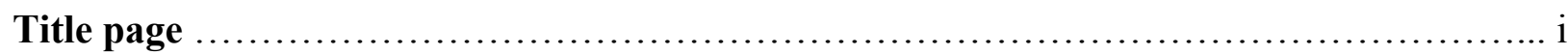

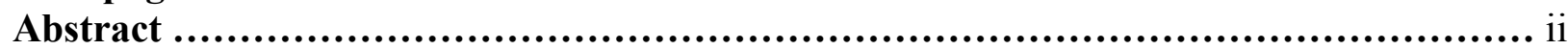

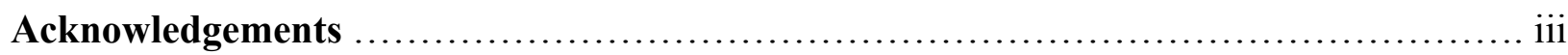

Chapter 1: Literature Review ........................................................

Part 1: Electrolyte transport in the gastrointestinal tract .....................................1

1.1 A brief introduction ............................................................

1.2 Basic structure of the intestinal epithelium ............................................

1.2.1 Electroneutral $\mathrm{NaCl}$ absorption.....................................

1.2.2 Short chain fatty acids and electroneutral $\mathrm{NaCl}$ absorption ................7

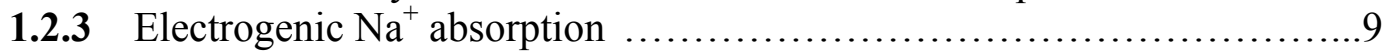

1.2.4 $\mathrm{Cl}^{-}$secretion .........................................................10

1.2.5 $\mathrm{K}^{+}$absorption and secretion .....................................13

Part 2: Physiological regulation of colonic electrolyte transport ............................... 14

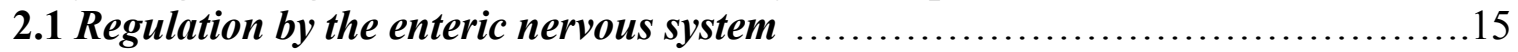

2.1.1 Major neurotransmitter system ..........................................16

Acetylcholine ................................................. 17

Vasoactive intestinal peptide ...........................................18

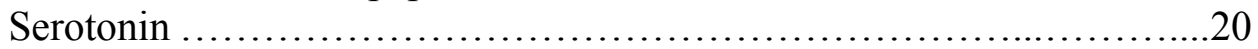

2.1.2 Neuro-immune communication ............................................22

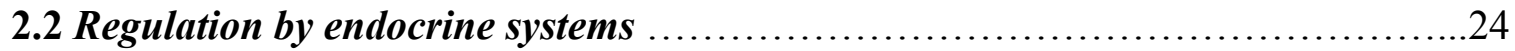

2.2.1 Regulation by corticosteroids ..........................................25

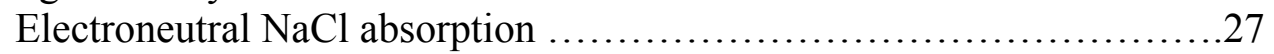

Electrogenic $\mathrm{Na}^{+}$absorption ...........................................30

$\mathrm{K}^{+}$transport .................................................... 31

Non-genomic effects ..................................................32

2.2.2 Regulation by somatostatin ......................................... 32

2.2.3 Regulation by sex steroids ................................................33

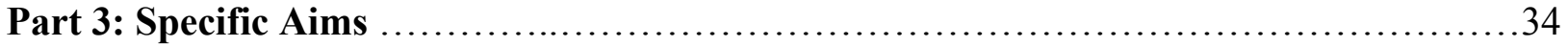

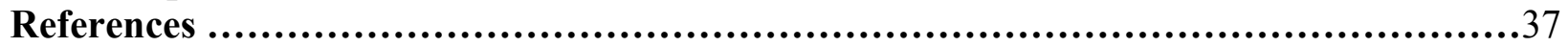

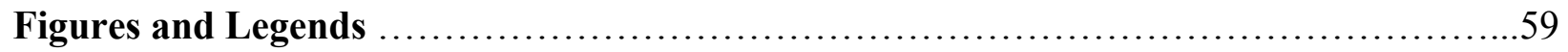

Chapter 2: "Activation of $\mathrm{KCNQ}\left(\mathrm{K}_{\mathrm{V}} 7\right) \mathrm{K}^{+}$channels in enteric neurons inhibits epithelial

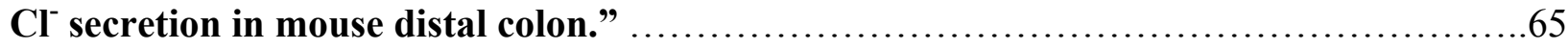

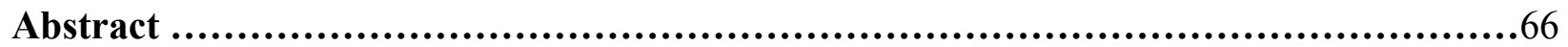

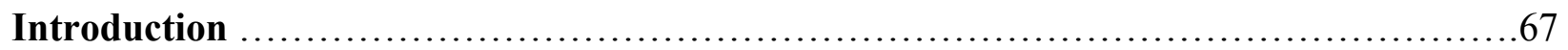

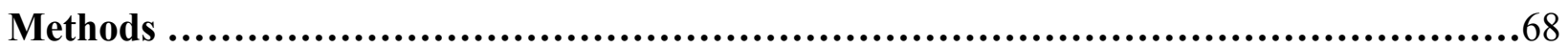

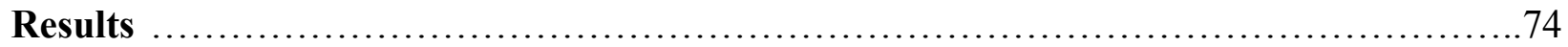

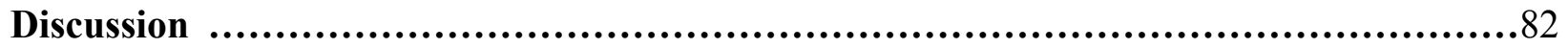

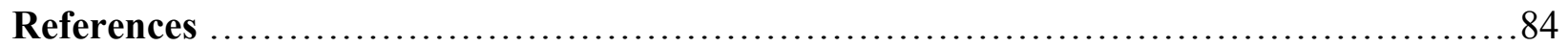

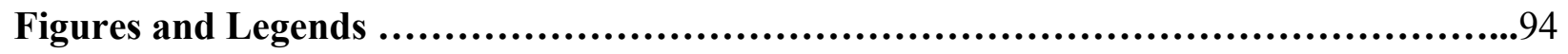

Chapter 3: "Flupirtine enhances NHE-3 mediated $\mathrm{Na}^{+}$absorption in rat colon via an ENS-

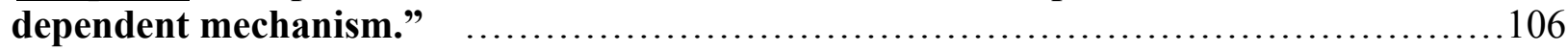




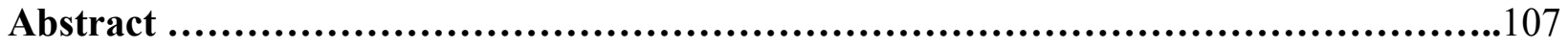

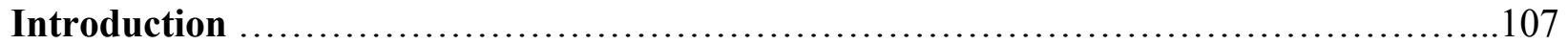

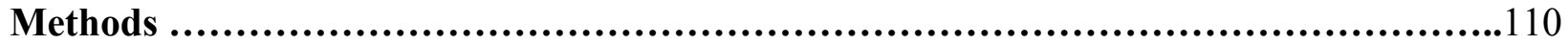

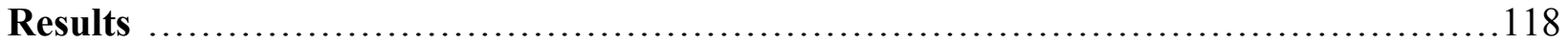

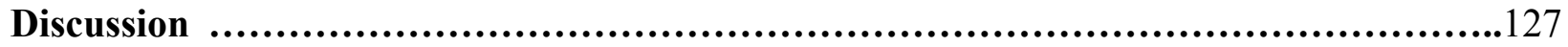

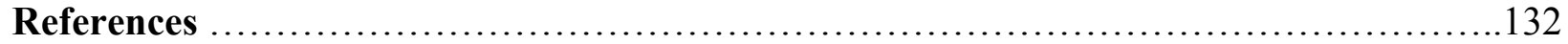

Tables ................................................................................138

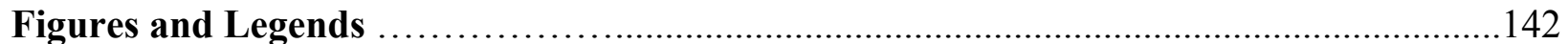

Chapter 4: "Aldosterone up-regulates basolateral $\mathrm{Na}^{+}-\mathrm{K}^{+}-2 \mathrm{Cl}^{-}$cotransporter-1 to support enhanced large conductance $\mathbf{K}^{+}$channel-mediated $\mathbf{K}^{+}$secretion in rat distal colon."

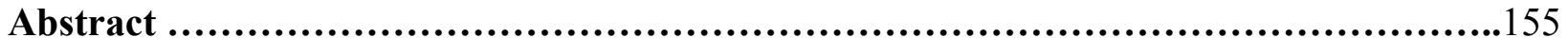

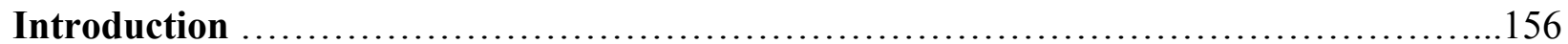

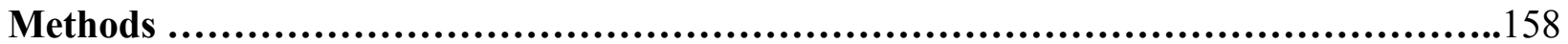

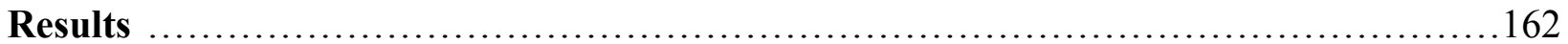

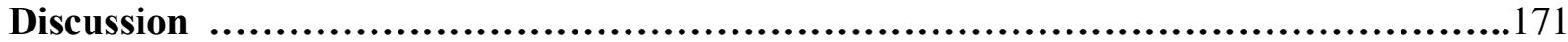

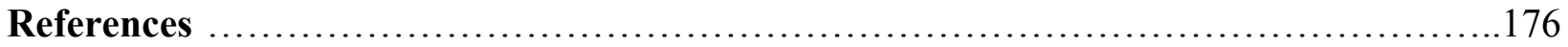

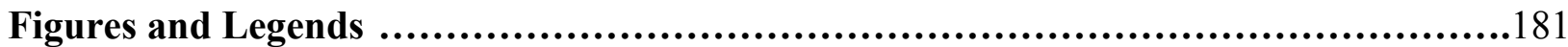

Chapter 5: "Aldosterone up-regulates NKCC1 expression and enhances electrogenic $\mathrm{Cl}^{-}$ secretion in rat proximal colon." ...........................................................197

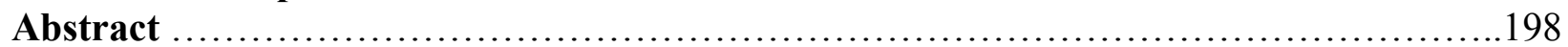

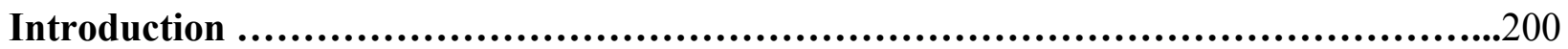

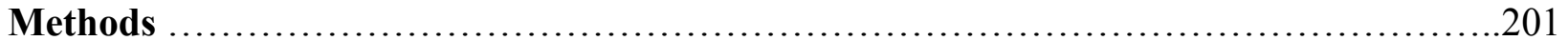

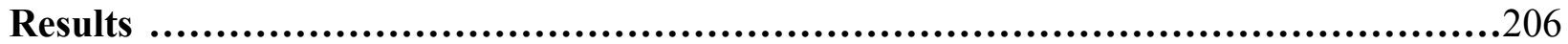

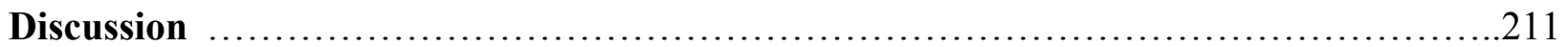

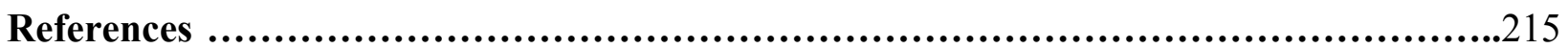

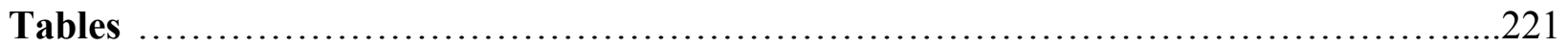

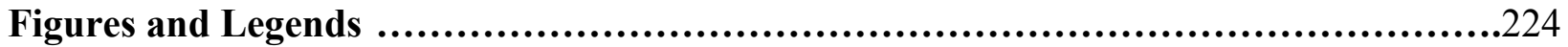

Chapter 6: General Discussion ...............................................................233

6.1 Novel role for KV7 channels in controlling ENS-dependent epithelial transport ...................................................................233

6.1.1 Brief summary of findings .....................................234

6.1.2 Remaining questions and future directions ...............................235

6.2 Characterization of the role of NKCC1 in aldosterone-stimulated $\mathrm{K}^{+}$secretion

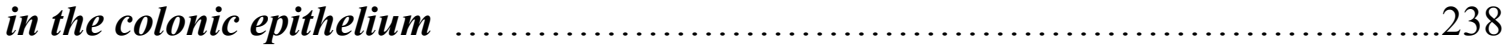

6.2.1 Brief summary of findings ...........................................239

6.2.2 Remaining questions and future directions ............................241

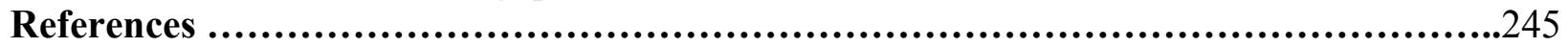

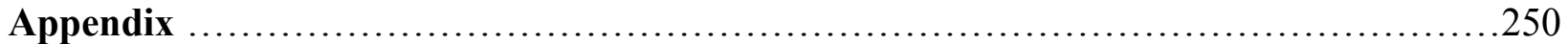




\section{CHAPTER 1: LITERATURE REVIEW}

\section{Part 1: electrolyte transport in the gastrointestinal tract.}

\subsection{A brief introduction}

Large organisms, including humans and other mammals, face the distinct biological challenge of supplying vital nutrients, water and oxygen to cells and tissues buried deep beneath a largely impenetrable body exterior. Simple diffusion of essential materials directly from our environment is not a viable option, as it is for our single-celled relatives. To address such a challenge, evolution has produced a complex arrangement of transport and exchange systems to allow for the safe and regulated uptake of these precious resources. A critical component to this process, the gastrointestinal (GI) tract - also known as the alimentary canal - is comprised of several distinct, yet inter-connected tissues whose primary function is the facilitation of water, nutrient and electrolyte (i.e., ion) transport into the body, and the simultaneous removal of waste materials and non-absorbed dietary substances $(1,2)$.

A wide variety of cell types are located within the alimentary canal, each of which is required to execute its many functions. Among these are: epithelial cells, which form the inner gut lining and carry out the transport of nutrients and electrolytes (3); layers of smooth muscle, which mix and propel material to aid in digestion and motility (4); vascular networks, carrying newlyabsorbed water and nutrients off to the their destinations within the body (5); neuronal networks that regulate epithelial transport, smooth muscle contraction and blood flow, while also communicating with central and peripheral nervous systems (6); and immune cells that constantly surveil the environment for potentially harmful materials or organisms (7). Beyond this, within the 
large intestine resides an entire community of microbes (often referred to as the microbiome) that outnumber the host's own cells 10:1 (8), and are essential for proper G.I. function $(9,10)$.

The focus of the studies within our laboratory has been to characterize and understand the mechanisms underlying the transport of electrolytes within the large intestine, which is just one of the many aspects of GI function. Appropriate absorption and/or secretion of osmotically active ions (a.k.a. "electrolytes"), such as sodium $\left(\mathrm{Na}^{+}\right)$, chloride $\left(\mathrm{Cl}^{-}\right)$or potassium $\left(\mathrm{K}^{+}\right)$, is paramount to maintaining fluid balance $(11,12)$. Coordinated transport of these ions creates the driving force for the movement of water into or out of the body, across the intestinal barrier. Consequently, perturbations in electrolyte transport can lead to severe, and even life-threating conditions (13). A review of the currently accepted principles of intestinal electrolyte transport will provide a useful context in which to present the findings from our recent studies, beginning with a discussion of the basic principles of ion transport within the gut.

For water to be transported from the lumen of the gut to the inside of the body, it must travel down an osmotic gradient. Water molecules cannot be transported directly, but will move across a (semi)permeable barrier to whichever compartment has a higher solute concentration (14). This phenomenon is referred to as osmosis and serves as the basis for all biological fluid transport (15). Therefore, absorption or secretion of solutes is the mechanism by which epithelial cells carry out their transport duties. In the intestine, this is mainly accomplished either by actively transporting $\mathrm{Na}^{+}$ions from inside the lumen across the epithelium and into the interstitial space, or by actively secreting $\mathrm{Cl}^{-}$into the lumen (1). Depending upon the region of the GI tract, a variety of other solutes may travel with $\mathrm{Na}^{+}$or $\mathrm{Cl}^{-}$and contribute to the generation of an osmotic gradient which favors water absorption or secretion (16). The details of the major absorptive and secretory 
pathways will be described in later sections. However, an understanding of the overall structure of intestinal epithelium is required to discern the function of its various transport mechanisms.

\subsection{Basic structure and function of the intestinal epithelium.}

The epithelium itself - the main constituent of the mucosa - has a particular spatial arrangement and cellular architecture to allow for vectorial (i.e., directional) ion transport. Simple columnar epithelial cells are situated adjacent to one another with their apical membranes facing the gut lumen and their basolateral membranes facing the interstitial space, or lamina propria. Histologically, the intestinal mucosa can generally be compartmentalized into absorptive surface and secretory crypt regions (17) (Fig. 1). At the base of each crypt are $\mathrm{LRG5}^{+}$stem cells which give rise to the other epithelial cell types along the crypt/surface axis (18). The cell populations inhabiting these two regions develop along a continuum as they traverse the crypt structure. Cells along the crypt/surface axis have separate physiological roles and biomolecular characteristics, which will be described later. With respect to an individual epithelial cell, the apical and basolateral membranes are also functionally distinct from one another (Fig. 2). These membranes are segregated, in part, by the presence of tight junction proteins that form a decisive boundary between the two domains (apical vs. basolateral regions) (19). Tight junctions also contribute to the formation of a restrictive extracellular barrier separating the lumen from the interstitial space. Forming a tight seal near the apical poles of adjacent epithelial cells prevents indiscriminate leaking of water, solutes or even pathogenic material (20) - any of which would undermine the absorptive process altogether.

Tight junctional complexes are formed by a number of different proteins, including the claudin and occludin gene families. These proteins extend through the plasma membrane and 
articulate with their counterpart proteins originating from adjacent cells, fortifying the epithelial monolayer and allowing very few substances to pass through (21). The pathway between epithelial cells and around tight junctions is termed the "paracellular" pathway and is quite restrictive in allowing the passage of most solutes - including ions. As a result, many epithelial ion transport pathways are described as "trans-cellular," passing through the intracellular compartment of the epithelial cell (Fig. 2). Trans-cellular solute absorption requires the coordinated actions of specialized transport proteins - ion channels or transporters - that are deliberately situated within the apical and/or basolateral membranes of absorptive epithelial cells. Specialized water channels, called aquaporins, are also required to allow the passage of water across the epithelial barrier (22), as tight junctions impede its movement through the para-cellular pathway. Aquaporin channel proteins are a heterogenous population with multiple gene family members (AQP1-12) differentially expressed in apical and basolateral membranes of many tissues. In the intestine $\mathrm{AQP} 3,4$ and 8 are the predominant isoforms located on in intestinal epithelial cells $(23,24)$ and trafficking to and from the plasma membrane is an important component in control the rate of water transport.

In the small intestine, absorptive epithelial cells reside in the numerous pillar-like structures called villi, while in the colon they are configured in flat surface regions that interface with the lumen (Fig. 1). In both cases, the absorptive domains (i.e. villus or surface epithelium) are interspersed with crypts Lieberkühn, where a separate population of secretory cells can be found. Fluid and electrolytes are transported both into and out of the gut lumen throughout the GI tract, as is necessary for certain digestive functions and hydration of the mucus layer that covers the epithelium (11). However, the main function of the colon is to desiccate fecal material by removing most of the remaining water content, as absorption of all dietary nutrients (amino acids, 
carbohydrates, fats and most minerals) is completed in the small intestine. In the colon, desiccation is accomplished by absorption of $\mathrm{Na}^{+}$via two predominant mechanisms: $\mathrm{Cl}^{-}$-dependent, electroneutral or $\mathrm{Cl}^{-}$-independent electrogenic absorption. Both of these processes occur primarily in absorptive surface colonocytes.

\subsubsection{Electroneutral $\mathrm{NaCl}$ absorption is mediated by coupled $\mathrm{Na}^{+} / \mathrm{H}^{+}$and $\mathrm{Cl}^{-} / \mathrm{HCO}_{3}^{-}$exchangers}

To understand a discussion of the mechanistic details of electrolyte transport, it is important to become at least somewhat familiar with one of the most common and useful experimental techniques in studying epithelial transport: Ussing chamber electrophysiology. In brief, when polarized tissues, such as the intestinal mucosa, are mounted in a chamber apparatus with separate mucosal (apical) and serosal (basolateral) baths and are voltage-clamped, this allows for the continuous measurement of an electrical parameter referred to as "short-circuit current", or I $\mathrm{SC}$ (25). ISC is defined as the amount of applied current necessary to maintain (i.e., clamp) the transepithelial voltage $\left(\mathrm{V}_{\mathrm{TE}}\right)$ to a potential of $0 \mathrm{mV}$, thus "short circuiting" the system. If left alone after mounting, intestinal mucosae will otherwise spontaneously develop this $\mathrm{V}_{\mathrm{TE}}$ as charged ions are moved across the tissue, resulting in a lumen-negative potential. This is typically on the order of 15 to $-30 \mathrm{mV}$ in the colon of most mammalian species. Measuring $\mathrm{I}_{\mathrm{SC}}$ provides a means of quantitating the total net electrogenic transport across a tissue, while at the same time eliminating the electromotive force imparted by the negative $\mathrm{V}_{\mathrm{TE}}$, if it were allowed to persist. Directional isotopic fluxes performed under these conditions therefore indicate the total active transport for a particular ion. Pharmacological treatments and/or bath solution ion substitution experiments have provided a great deal of information regarding the identity and properties of many colonic epithelial transport proteins. 
Early studies by Binder and colleagues using radioisotope $\left({ }^{22} \mathrm{Na}^{+}\right.$and $\left.{ }^{36} \mathrm{Cl}^{-}\right)$flux experiments performed in rat colon under voltage-clamped conditions led to the identification of electroneutral and electrogenic $\mathrm{Na}^{+}$absorptive mechanisms (26,27). A large component of $\mathrm{Na}^{+}$ absorption was found to be dependent on the presence of $\mathrm{Cl}^{-}$in the apical tissue bath. In addition, net $\mathrm{Na}^{+}$and $\mathrm{Cl}^{-}$fluxes were found to be greater than the $\mathrm{I}_{\mathrm{SC}}$ generated by the tissues during these experiments, indicating that much of the active $\mathrm{Na}^{+}$absorption was electroneutral (27). Foster et al first identified the presence of a $\mathrm{Na}^{+} / \mathrm{H}^{+}$exchange process in the apical membranes of rat colonocytes that brings a $\mathrm{Na}^{+}$ion into the cell across the apical membrane in exchange for a proton (28). Later, functional coupling to a $\mathrm{Cl}^{-} / \mathrm{HCO}_{3}{ }^{-}$exchange system via a $\mathrm{pH}$-dependent mechanism was discovered (29). Intracellular $\mathrm{pH}\left(\mathrm{pH}_{\mathrm{i}}\right)$ rises as protons are transferred out of the cell in exchange for $\mathrm{Na}^{+}$. Outward $\mathrm{HCO}_{3}{ }^{-}$efflux occurs to offset this $\mathrm{pH}_{\mathrm{i}}$ change, and $\mathrm{Cl}^{-}$is brought into the cell in return. This parallel $\mathrm{Na}^{+} / \mathrm{H}^{+}$and $\mathrm{Cl}^{-} / \mathrm{HCO}_{3}{ }^{-}$exchange system can also be found in the apical membranes of the small intestine $(30,31)$ and proximal tubule of the kidney $(32)$. It accounts for the majority of whole-body $\mathrm{NaCl}$ and water absorption among mammals.

In the colon, apical $\mathrm{Na}^{+} / \mathrm{H}^{+}$exchange is primarily attributable to the activity of $\mathrm{Na}^{+} / \mathrm{H}^{+}$ exchanger isoform 3 (NHE-3; Slc9a3), with some involvement of NHE-2 (Slc9a2) (33) (Fig. 3A). Apical $\mathrm{Cl}^{-} / \mathrm{HCO}_{3}{ }^{-}$exchange is mediated by a gene called "down-regulated in adenoma" (DRA: Slc26a3) (34,35) (Fig. 3A), and possibly putative anion transporter 1 (PAT1; Slc26a6), although its activity appears to be restricted to the small intestine (36). $\mathrm{Cl}^{-} / \mathrm{OH}^{-}$exchange may also be coupled to $\mathrm{Na}^{+} / \mathrm{H}^{+}$exchange via a $\mathrm{pH}$-dependent, $\mathrm{HCO}_{3}^{-}$-independent mechanism $(37,38)$. However, the identity of a $\mathrm{Cl}^{-} / \mathrm{OH}^{-}$exchanger in the colon is yet undetermined. Genetic disruption of Slc26a3 (DRA) produces severe diarrhea in mice (34). In humans, several diarrheal diseases, such as congenital sodium or congenital chloride diarrhea, are the result of mutations in neutral 
$\mathrm{Na}^{+} / \mathrm{H}^{+}$(NHE-3) or $\mathrm{Cl}^{-} / \mathrm{HCO}_{3}{ }^{-}$(DRA) exchangers, respectively $(39,40)$. These conditions are very rare and often present with a severe disease phenotype.

Coupled $\mathrm{Na}^{+} / \mathrm{H}^{+}$and $\mathrm{Cl}^{-} / \mathrm{HCO}_{3}{ }^{-}$exchangers mediate $\mathrm{Na}^{+}$and $\mathrm{Cl}^{-}$uptake at the apical membranes of epithelial cells (41). However, the driving force generating the electrochemical gradient that allows for sustained $\mathrm{Na}^{+}$entry (and $\mathrm{Cl}^{-}$entry that is coupled to it) is established by the continuous activity of the basolateral $\mathrm{Na}^{+} / \mathrm{K}^{+}$ATPase (a.k.a. the $\mathrm{Na}^{+} / \mathrm{K}^{+}$pump), which functions to extrude $3 \mathrm{Na}^{+}$across the basolateral membrane in exchange for $2 \mathrm{~K}^{+}$(11) (Fig. 3A). $\mathrm{Na}^{+}$extrusion via $\mathrm{Na}^{+} / \mathrm{K}^{+}$ATPase occurs continuously and is sensitive to cardiac glycosides, such as ouabain (42). Though costly to operate, this enzyme serves the dual purpose of maintaining $\mathrm{Na}^{+}$ at low intracellular levels and providing a pathway across the basolateral membrane for $\mathrm{Na}^{+}$to be taken up into the blood. $\mathrm{Cl}^{-} / \mathrm{HCO}_{3}{ }^{-}$exchange occurs secondarily to $\mathrm{Na}^{+} / \mathrm{H}^{+}$exchange and both are ultimately dependent on $\mathrm{Na}^{+} / \mathrm{K}^{+}$ATPase activity. As intracellular $\mathrm{Cl}^{-}$ions accumulate, basolateral $\mathrm{Cl}^{-} / \mathrm{HCO}_{3}{ }^{-}$exchange via anion exchanger $2(\operatorname{Slc} 4 a 2)(43)$, basolateral $\mathrm{K}^{+} / \mathrm{Cl}^{-}$co-transporter $(\mathrm{KCC}$; Slc12a4) (44) and parallel intermediate conductance $\mathrm{K}^{+}$(IK; Kcnn4) channel and CLC-2 (clcn2) $\mathrm{Cl}^{-}$channel proteins (45) can all facilitate $\mathrm{Cl}^{-}$efflux into the interstitial space, completing the $\mathrm{NaCl}$ absorption process (Fig. 3A).

\subsubsection{Electroneutral NaCl absorption is also coupled to short-chain fatty acid absorption}

Short-chain fatty acids (SCFAs) are produced via the metabolism of non-absorbed dietary carbohydrates by commensal bacteria in the colon (46). Acetate, propionate and butyrate are the predominant SCFA species generated, and their total concentration within the gut lumen can be upwards of $100 \mathrm{mM}$ (47). Among these species, butyrate has received the most investigative attention because it not only provides a major fuel source for colonocytes (46), but also heavily 
influences gene expression by acting as a histone deacetylase (HDAC) inhibitor $(48,49)$. The absorptive pathways of $\mathrm{Na}^{+}$and butyrate are unquestionably linked. Butyrate significantly enhances electroneutral $\mathrm{NaCl}$ uptake and butyrate uptake is dependent on $\mathrm{Na}^{+}$as well $(50,51)$. Still, the exact pattern for butyrate-dependent $\mathrm{NaCl}$ absorption has not been fully elucidated at this point in time.

Monocarboxylate transporters (MCTs) are widely accepted as the major butyrate cellular intake mechanism in a variety of cell types (52). These transporters are dependent on either an inward $\mathrm{H}^{+}$gradient (MCT-1; Slc16al) or inward $\mathrm{Na}^{+}$(sodium) gradient (MCT-1; Slc5a8). Both proteins have been localized to the colonic epithelium via immunolabeling studies $(53,54)$. However, the involvement of additional butyrate transport pathways is likely. Functional evidence points to $\mathrm{Na}^{+}$-dependent butyrate requiring an outward-directed $\mathrm{HCO}_{3}^{-}$gradient (55), and is not necessarily related directly to $\mathrm{pH}_{\mathrm{i}}$, as would be expected for $\mathrm{H}^{+}$-coupled transport via MCT-1. The involvement of SMCT-1 in butyrate uptake is also brought into question because its $K_{M}$ value for butyrate is very high - nearly $50 \mathrm{mM}$ (52). Studies using isolated apical membrane vesicles suggest that $\mathrm{Cl}^{-}$/butyrate and $\mathrm{HCO}_{3}{ }^{-}$/butyrate exchangers are both present on the apical membranes of surface epithelial cells, and operate in parallel to each other (56). However, the molecular identity of these putative anion antiporters has not been elucidated as of yet.

Operation of $\mathrm{Cl}^{-}$/butyrate or $\mathrm{HCO}_{3}{ }^{-}$/butyrate transport systems is functionally coupled to the $\mathrm{Na}^{+} / \mathrm{H}^{+}$exchangers described in the previous section. NHE-2/NHE-3 dependent $\mathrm{Na}^{+}$absorption is augmented in the presence of butyrate, even without $\mathrm{HCO}_{3}{ }^{-}$present in the apical bath, but to a greater extent when both anions are present $(51,57)$. An outward-directed $\mathrm{HCO}_{3}{ }^{-}$gradient likely drives a $\mathrm{HCO}_{3}{ }^{-} /$butyrate exchanger to bring butyrate into the cell across the apical membrane. The resultant fall in $\mathrm{pH}_{\mathrm{i}}$ triggers activation of apical NHE-2/NHE-3. Finally, intracellular butyrate is 
exchanged for $\mathrm{Cl}^{-}$across the apical membrane, completing the cycle. While apical butyrate- and $\mathrm{HCO}_{3}{ }^{-}$-dependent neutral $\mathrm{Na}^{+}$absorptive mechanisms are interconnected, they each can function independently as well. In addition, NHE-2 and NHE-3 were shown to differentially regulate butyrate-dependent $\mathrm{Na}^{+}$absorption in healthy versus inflamed colon (58), with NHE-2 mediated, butyrate-dependent $\mathrm{Na}^{+}$absorption predominating under inflammatory conditions.

\subsubsection{Electrogenic $\mathrm{Na}^{+}$absorption is mediated by the epithelial $\mathrm{Na}^{+}$channel; $\mathrm{ENaC}$.}

Neutral $\mathrm{NaCl}$ absorption occurs throughout most of the small and large intestine. In some species, including rabbit, mouse and human, the distal segment of the colon is also characterized by an electrogenic $\mathrm{Na}^{+}$absorptive pathway that is not dependent on the presence of luminal $\mathrm{Cl}^{-}$ $(1,11)$. This is mediated by an apical membrane epithelial $\mathrm{Na}^{+}$channel (ENaC) (Fig. 3B), first described functionally by Foster et. al. (27), who observed that net ${ }^{22} \mathrm{Na}^{+}$absorption was roughly equivalent to the $\mathrm{I}_{\mathrm{SC}}$ (i.e., was entirely electrogenic) measured across colonic epithelial sheets under certain conditions. The channel carrying this current is now known to be encoded by the Scnn gene family. ENaC channels are comprised of three highly homologous subunits (ENaC $\alpha, \beta, \gamma ;$ Scnna, Scnnb, Scnng) which are transcriptionally and post-translationally regulated by a variety of factors (59) (more later).

Apical ENaC-mediated $\mathrm{Na}^{+}$influx is also driven by basolateral $\mathrm{Na}^{+} / \mathrm{K}^{+}$ATPase activity (Fig. 3B), which produces an electrochemical gradient favoring $\mathrm{Na}^{+}$entry from the lumen, similar to what has been described for electroneutral absorption. Unlike NHE-3-mediated absorption however, opening of $\mathrm{ENaC}$ channels generates an electrical current because no other charged solutes are transported in exchange. $\mathrm{ENaC}$ is very sensitive to inhibition by the diuretic, amiloride $\left(\mathrm{IC}_{50} \sim 100 \mathrm{nM}\right)(59)$. Application of $10 \mu \mathrm{M}$ amiloride dramatically inhibits both $\mathrm{I}_{\mathrm{SC}}$ and ${ }^{22} \mathrm{Na}^{+}$ 
absorption in colonic tissues with high ENaC activity (60). NHE-3 and NHE-2 are also inhibited by amiloride but are much less sensitive, with $\mathrm{IC}_{50}$ values in the hundred $\mu \mathrm{M}$ range. Both NHE-3 and $\mathrm{ENaC}$-mediated $\mathrm{Na}^{+}$absorption take place primarily in surface epithelial cells of the colon, although there is some evidence of neutral $\mathrm{Na}^{+}$absorption within the crypt regions under certain experimental conditions (61).

Both electroneutral and electrogenic $\mathrm{Na}^{+}$absorptive processes are present in the colon under normal physiological conditions. In many species, including humans, mice and rats, ENaCmediated absorption becomes more prominent in the distal regions of the colon and rectum (1). NHE-2/3 mediated electroneutral absorption is the primary mechanism within the proximal segment of the colon (62), as well as in the small intestine (33). The gradual transition from electroneutral to electrogenic $\mathrm{Na}^{+}$absorption from proximal to distal regions bears resemblance to $\mathrm{Na}^{+}$transport within the kidney as well, as neutral $\mathrm{Na}^{+}$absorption predominates tubular transport until urine reaches the collecting duct (32). Many diseases related to mutations in $\mathrm{ENaC}$, such as Liddle syndrome and pseudo-hyperaldosteronism, present with disturbances in renal function (63). In the colon, $\mathrm{ENaC}$ plays a role mostly in the final desiccation of fecal material, aiding in the final removal of its water content in preparation for defecation.

\subsection{4 $\mathrm{Cl}^{-}$secretion is electrogenic and occurs within the crypt}

The colon also possesses the ability transport fluid into the lumen by secreting $\mathrm{Cl}^{-}$across the apical membranes of crypt epithelial cells (64). $\mathrm{Cl}^{-}$is the electrolyte generally associated with fluid secretion, whereas $\mathrm{Na}^{+}$is associated with fluid absorption. This is because the

electrochemical driving forces heavily favor $\mathrm{Na}^{+}$entry into the epithelial cell as a result of continuous $\mathrm{Na}^{+} / \mathrm{K}^{+}$ATPase activity maintaining very low $\mathrm{Na}^{+}$levels inside the cell. Efflux of $\mathrm{Na}^{+}$ 
against its electrochemical gradient toward the lumen is nearly impossible from a biophysical standpoint. Intracellular $\mathrm{Cl}^{-}$concentrations are not as rigorously maintained because apical and basolateral membranes are relatively permeable to the ion. Consequently, $\mathrm{Cl}^{-}$can readily move into or out of the epithelial cell in response to the activities of other transport proteins.

The cystic fibrosis transmembrane conductance regulator (CFTR; Cftr) is an apical membrane-localized $\mathrm{Cl}^{-}$channel protein found in cells within the crypt region $(65,66)$ (Fig. 3C). The channel protein was first identified as the defective gene product associated with cystic fibrosis (CF) (67), an inherited condition characterized by inadequate hydration of airway epithelia that leads to impaired mucociliary clearance and persistent, life-threatening bacterial infections. Patients with cystic fibrosis have a small disruption in the gene (usually a deletion of phenylalanine 508; $\Delta \mathrm{f508)}$ that results in non-functional CFTR channels because of inappropriate membrane trafficking (68). Normally, opening of CFTR channels in the colon is triggered by cyclic AMP (cAMP)-dependent phosphorylation in response to any number of stimuli, including stimulation by enteric nervous system inputs or the presence of pathogen-derived substances, such as cholera toxin (69).

CFTR mediates most of, if not all luminal $\mathrm{Cl}^{-}$secretion in the colon, as $\mathrm{CF}$ mice fail to generate any $\mathrm{Cl}^{-}$secretory response to physiological agonists (70). CFTR contributes significantly to the basal and/or stimulated $\mathrm{I}_{\mathrm{SC}}$ observed in most GI tissues because $\mathrm{Cl}^{-}$efflux through the channel is also electrogenic. There is some controversy regarding the participation of other apical membrane $\mathrm{Cl}^{-}$channels, including the $\mathrm{Ca}^{2+}$-activated TMEM16A (Anol) (71) (Fig. 3C), and conflicting results have been produced using pharmacological and transgenic animal models (72,73). In the absence of CFTR activity via genetic knockout (73) or drug inhibition (74), basal and agonist (e.g., cAMP)-stimulated $\mathrm{Cl}^{-}$secretion is essentially absent. Many newborn CF patients 
are also affected by a condition known as meconium ileus, where non-functional CFTR channels in the GI tract results in poor hydration of the gut lumen and subsequent buildup of fecal material (75).

Basolateral $\mathrm{Na}^{+} / \mathrm{K}^{+} / 2 \mathrm{Cl}^{-}$co-transporter (NKCC1; Slc12al) supports CFTR-dependent $\mathrm{Cl}^{-}$ secretion in the crypts of the large intestine $(11,76)$ (Fig. 3C). NKCC1 brings these ions across the basolateral membrane in this stoichiometry $(1: 1: 2)$ by harnessing the inward $\mathrm{Na}^{+}$gradient established by the $\mathrm{Na}^{+} / \mathrm{K}^{+}$ATPase. To allow sustained $\mathrm{Cl}^{-}$efflux across the apical membrane, $\mathrm{Na}^{+}$ and $\mathrm{K}^{+}$are immediately recycled back across the basolateral membrane through the $\mathrm{Na}^{+} / \mathrm{K}^{+}$ ATPase and $\mathrm{K}^{+}$channels, respectively (77). The latter play a particularly critical role by maintaining the electromotive force for $\mathrm{Cl}^{-}$efflux via depolarization as $\mathrm{K}^{+}$ions exit the cell. Intermediate conductance (IK; Kcnn4) (78) and voltage-gated subfamily Q member $1\left(\mathrm{~K}_{\mathrm{V}} 7.1\right.$; Kcnq1) (79) $\mathrm{K}^{+}$channels have been shown to support CFTR-mediated efflux, as drug inhibition or genetic ablation of either channel substantially reduces the capacity for $\mathrm{Cl}^{-}$secretion. Expression of CFTR, NKCC1, IK and $\mathrm{K}_{\mathrm{V}} 7.1$ is localized primarily to cells within the crypt region, making this the principal location for fluid secretion in the colon (Fig. 3C).

Closely related to the secretion of $\mathrm{Cl}^{-}$is electrogenic $\mathrm{HCO}_{3}{ }^{-}$secretion, which most frequently is mediated by canonical $\mathrm{Cl}^{-}$channels, including $\mathrm{CFTR}(80)$. $\mathrm{HCO}_{3}{ }^{-}$permeability through the CFTR channel is $\sim 25 \%$ that of $\mathrm{Cl}^{-}(80)$ and is thus a major carrier of anionic current in the epithelium. CFTR-mediated $\mathrm{HCO}_{3}{ }^{-}$secretion is critical for proper release and functionality of Mucin proteins secreted by Goblet cells (81), which help to form a protective mucous lining on the surface of the epithelium. While it remains unclear whether Goblet cells express CFTR, another $\mathrm{Ca}^{2+}$-activated anion channel Bestrophin-2 (BEST-2; Best2), is highly expressed specifically in Goblet cells and is critical for $\mathrm{HCO}_{3}{ }^{-}$secretion in the colon (82). $\mathrm{HCO}_{3}{ }^{-}$secretion mediated by 
BEST-2 may also be necessary for protection of the epithelium from luminal microbes, as Best $2^{-/-}$ mice display increased sensitivity to DSS-induced colitis (82).

\subsection{5 $\mathrm{K}^{+}$is both actively absorbed and secreted in the colon}

$\mathrm{Na}^{+}$and $\mathrm{Cl}^{-}$are transported in the colon for the purpose of generating osmotic forces to drive fluid absorption and/or secretion. On the other hand, vectorial colonic $\mathrm{K}^{+}$transport plays a role in overall $\mathrm{K}^{+}$homeostasis within the body $(83,84)$. Blood $\mathrm{K}^{+}$levels are very tightly regulated because even slight alterations in extracellular $\mathrm{K}^{+}$can be fatal. The colon serves as a complement to the kidney in terms of taking in or eliminating excess $\mathrm{K}^{+}$from the luminal contents in an effort to maintain blood $\mathrm{K}^{+}$levels $(85,86)$. Depending on the needs of the organism, the colon has the capacity to either actively absorb or secrete $\mathrm{K}^{+}(83)$. This is in sharp contrast to $\mathrm{K}^{+}$transport in the small intestine, which is believed to occur only passively due to the mass absorption of water itself in a process called solvent drag (87). It should be noted that the basolateral $\mathrm{K}^{+}$recycling necessary for $\mathrm{Cl}^{-}$secretion is not considered as vectorial transport because $\mathrm{K}^{+}$ions are continuously moved back and forth across the basolateral membrane and do not enter or exit the body (i.e., transport is not unidirectional).

Active $\mathrm{K}^{+}$absorption is mediated by apical membrane $\mathrm{H}^{+} / \mathrm{K}^{+}$ATPases (ATP12al) (Fig. 3B) that can be either sensitive or insensitive to the cardiac glycoside, ouabain (88). These $\mathrm{H}^{+} / \mathrm{K}^{+}$ ATPases share about $60 \%$ homology with the basolateral $\mathrm{Na}^{+} / \mathrm{K}^{+}$ATPase and all three transporters are inhibited by the P-type ATPase inhibitor, $\mathrm{Na}^{+}$-orthovanadate $(89) . \mathrm{H}^{+} / \mathrm{K}^{+}$ATPases are expressed by surface epithelial cells only in the distal colon, as determined by uptake studies using isolated apical membrane vesicles (88). $\mathrm{K}^{+}$efflux across the basolateral membrane is facilitated by IK channels functioning in parallel to CLC-2 $\mathrm{Cl}^{-}$channels (45), or by the $\mathrm{K}^{+} / \mathrm{Cl}^{-}$co-transporter 
(KCC) (44). Active $\mathrm{K}^{+}$absorption in the colon is therefore an electroneutral process. Besides providing a pathway for $\mathrm{K}^{+}$uptake into the body, $\mathrm{H}^{+} / \mathrm{K}^{+}$ATPase activity may support electrogenic (ENaC-mediated) $\mathrm{Na}^{+}$absorption by supporting $\mathrm{K}^{+}$recycling at the apical membrane to help sustain the electrochemical driving force necessary for continuous $\mathrm{Na}^{+}$entry (90). Evidence supporting this model is limited, however. A more apparent role for the colonic $\mathrm{H}^{+} / \mathrm{K}^{+}$ATPase in regulating whole-body $\mathrm{K}^{+}$balance is evidenced by the finding that deletion of functional colonspecific $\mathrm{H}^{+} / \mathrm{K}^{+}$ATPase in mice resulted in an increased sensitivity to dietary $\mathrm{K}^{+}$deprivation (91).

While absorption is electroneutral, colonic $\mathrm{K}^{+}$secretion is electrogenic and is mediated by apical membrane large conductance (BK; Kcnmal) $(92,93)$, with possible involvement of intermediate conductance (IK) $\mathrm{K}^{+}$channels (94) (Fig. 3B). Uptake of $\mathrm{K}^{+}$to support apical efflux is mediated by basolateral $\mathrm{Na}^{+} / \mathrm{K}^{+}$ATPase and $\mathrm{NKCC} 1$ activity (86). There is evidence supporting both crypt $(95)$ and surface $(96,97)$ localization of electrogenic $\mathrm{K}^{+}$secretion systems, and species variation likely exists as well $(98,99)$. Active $\mathrm{K}^{+}$secretion is present throughout the entire large intestine, whereas absorption occurs only in the distal segment (83). Colonic $\mathrm{K}^{+}$secretion provides an alternate outlet for $\mathrm{K}^{+}$, in addition to the kidney. This pathway is important for preventing lifethreating hyperkalemia in patients with end-stage renal disease $(100,101)$. Excessive $\mathrm{K}^{+}$secretion has also been implicated in ulcerative colitis (UC) (96) and colonic pseudo-obstruction (102). Both of these conditions are associated with increased stool $\mathrm{K}^{+}$content which is likely the result of enhanced BK-mediated $\mathrm{K}^{+}$secretion.

\section{Part 2: Physiological regulation of colonic electrolyte transport}

The process of digestion requires a great deal of fluid secretion from various sources into the small intestine for proper enzymatic and digestive function. These secretions originate from 
the small intestine itself, as well as salivary, pancreatic and biliary secretions carrying essential factors like $\mathrm{HCO}_{3}{ }^{-}$, enzymes and bile (3). Nutrients are broken down and absorbed along with $\mathrm{Na}^{+}$, $\mathrm{Cl}^{-}$, and $\mathrm{K}^{+}$as the food products (by this point, referred to as chyme) travel along the length of the small intestine. The majority of all water presented to the GI tract is also absorbed in the small intestine under normal conditions. Over the course of a normal day for the average adult, the small intestine typically absorbs between 6 and $7 \mathrm{~L}$ of fluid, with about $2 \mathrm{~L}$ remaining, which enters the colon. Another 1.8 - 1.9L is absorbed by the colon in the process of desiccation (103). However, the colon has a large overhead capacity for water absorption (up to 5L), as well as secretion (e.g., in the case of enterobacterial infection). The colon is therefore a very dynamic absorptive tissue. Regulation of colonic electrolyte and water transport is of critical importance in maintaining fluid balance. This is achieved by a host of different factors, including neuronal and hormonal influences.

\section{1 - Regulation of colonic ion transport by the enteric nervous system.}

The enteric nervous system (ENS) is a vast network of neurons and glia located entirely within the GI tissue. More than 100 million neurons constitute the ENS, which can operate independent of inputs from other central or peripheral neural networks $(6,104)$, although significant crosstalk does occur in vivo. The ENS is macroscopically organized into two main plexuses: the myenteric (Auerbach's) plexus - located between inner and outer smooth muscle layers, and the submucosal (Meissner's) plexus - located beneath the lamina propria and muscularis mucosae in the submucosal space. The myenteric and submucosal plexuses are generally considered to control smooth muscle contraction/motility and epithelial secretion/absorption, respectively (105). 
Sensory afferents also permeate the GI tissue to gather information about luminal contents, $\mathrm{pH}$ of the epithelial environment, gut wall distension, presence of foreign substances and other stimuli $(106,107)$. This information is carried via ascending pathways, often to both CNS and ENS divisions, which in turn modulate epithelial transport functions (108). There is a staggering complexity in the anatomical and functional articulations between intrinsic ENS neurons (located entirely within the GI tissue, e.g., myenteric/submucosal plexuses) and extrinsic neurons, whose cell bodies reside outside of the GI tissue (e.g., spinal afferents, sympathetic and parasympathetic fibers) (104).

\subsection{1 - Major neurotransmitter systems mediating neuronal-epithelial communication}

Within the ENS exist many subpopulations of neurons that directly control smooth muscle or epithelial activity, collectively known as "secretomotor" neurons (109). These are connected to sensory neurons, interneurons and glia to form an expansive network capable transducing sensory input directly from the GI tissue and generating a secretory and/or motor response to a stimulus (110). Secretomotor neurons of the submucosal plexus are the primary effector cells of this reflex circuit with control over epithelial transport (111). These neurons, which typically release prosecretory neurotransmitters (a.k.a., secretagogues) to the epithelium, are often localized to submucosal ganglia with projections in close contact to the mucosa. Further innervation is also provided by divisions of the Vagus nerve, a major component to the parasympathetic system (112). More than 30 different common neurotransmitters have been identified in ENS neurons, including (but not limited to) acetylcholine, vasoactive intestinal peptide (VIP), and serotonin (5hydroxytryptamine; 5-HT) $(6,113)$. Acetylcholine and VIP are the most abundant ENS-derived neurotransmitters in the large intestine that heavily influence epithelial secretions $(113,114)$. Serotonin plays an important role in the conveyance of sensory information retrograde from the 
epithelium to primary sensory neurons, as well as in peristalsis - the coordinated contractions that propel luminal contents onward through the intestine - which frequently occurs together with epithelial secretions (115). Each of these transmitter systems will be discussed below.

\section{Acetylcholine}

Acetylcholine (ACh) is among the most abundant signaling molecules in the body and is vitally important for both neuro-neuronal and neuro-epithelial communication in the gut $(110,116)$. Ganglionic transmission (i.e., neuro-neuronal communication) in most peripheral neural pathways is mediated by ACh. The ENS is no exception to this, as nicotinic ACh receptors (nAChRs) are widely expressed throughout $(117,118)$. Inhibition of nicotinic transmission has a profound effect on secretomotor ENS function, highlighting the importance of cholinergic pathways in the gut (119). Activation of neuron-localized nAChRs triggers the propagation of action potentials through descending pathways until the effector secretomotor neurons are activated. When ACh is delivered to the colonic epithelium via cholinergic secretomotor neurons, it primarily binds to and activates $\mathrm{M}_{3}$ muscarinic receptors, which are expressed ubiquitously on the basolateral membranes of colonocytes along the entire crypt axis $(120,121) . \mathrm{M}_{1}$ muscarinic receptors are also expressed on epithelial cell basolateral membranes (122), as well as post-synaptic membranes in ENS neurons (123), although their involvement in epithelial signaling is less than that of the $\mathrm{M}_{3}$ receptor subtype.

$\mathrm{M}_{3}$ receptors in the colonic epithelium are coupled to $\mathrm{G}_{\mathrm{aq}}$ proteins that trigger a rapid increase in cytosolic $\mathrm{Ca}^{2+}$ when activated, through release of $\mathrm{Ca}^{2+}$ from ER stores that is mediated by inositol triphosphate $\left(\mathrm{IP}_{3}\right)(121)$. This robust, but transient $\mathrm{Ca}^{2+}$ event leads to a number of outcomes related to ion transport. Opening of $\mathrm{Ca}^{2+}$-activated $\mathrm{K}^{+}$channels on the crypt cell 
basolateral membrane creates an immediate electromotive force favoring apical $\mathrm{Cl}^{-}$efflux through CFTR and other $\mathrm{Cl}^{-}$channels, which may also be activated directly by $\mathrm{Ca}^{2+}(124,125)$. At the same time, $\mathrm{NKCC} 1$ is immediately inserted into the basolateral membrane from sub-membrane vesicles in response to $\mathrm{M}_{3}$ receptor activation to allow for enhanced $\mathrm{Cl}^{-}$uptake (126). $\mathrm{Ca}^{2+} /$ calmodulinactivated PKC has also been shown to regulate basolateral $\mathrm{K}^{+}$channel activation via direct phosphorylation (127), while indirect $\mathrm{Ca}^{2+}$-dependent activation of PKA leads to phosphorylation of NKCC1 $(126,128)$, supporting maximal $\mathrm{Cl}^{-}$efflux in this context. Further, Goblet cells that secrete mucus and $\mathrm{HCO}_{3}{ }^{-}$are also sensitive to cholinergic input $(129,130)$ and when stimulated, release large amounts of $\mathrm{HCO}_{3}{ }^{-}$via CFTR or possibly Bestrophin channels (82).

Muscarinic receptors are also found in the surface epithelial cells as well. Cholinergic input in this region of the tissue leads to the rapid internalization of NHE-3 from the apical membrane (131). This process is dependent on phospholipase C (PLC; activated by muscarinic stimulation) and involves extensive rearrangement of the large NHE-3 macromolecular complex, as well as cytoskeletal elements $(132,133)$. Termination of $\mathrm{Na}^{+}$uptake prevents the concurrent uptake of $\mathrm{Cl}^{-}$ through apical $\mathrm{Cl}^{-} / \mathrm{HCO}_{3}{ }^{-}$exchangers (e.g., DRA) because the activity of these transporters is coupled by intracellular $\mathrm{pH}_{\mathrm{i}}$ (134). Inhibition of absorptive processes in surface cells, along with stimulation of anion secretion within the crypt, combine to produce a powerful secretory response to cholinergic input to the epithelium.

\section{Vasoactive Intestinal Peptide (VIP)}

The other predominant secretory neurotransmitter in the large intestine of most mammalian species is VIP $(113,116)$. VIP is a 28 -residue amino acid peptide produced by secretomotor neurons in the ENS, but is also found in other populations within the ENS (inhibitory motor 
neurons, interneurons), as well as the CNS (135). In the colon, VPAC1 receptors (which bind VIP) are expressed by crypt epithelial cells and located on the basolateral membrane (136). These receptors are coupled to $\mathrm{G}_{\mathrm{s}}$ and their activation leads to production of cAMP by adenylate cyclase, the target of $\mathrm{G}_{\mathrm{s}}$ proteins. cAMP-dependent protein kinase A (PKA) activation leads to the phosphorylation of apical membrane $\mathrm{CFTR} \mathrm{Cl}^{-}$channels $(137,138)$ and cAMP-activated $\mathrm{K}^{+}$ $\left(\mathrm{K}_{\mathrm{V}}\right.$ 7.1) channels on the basolateral membrane (139) to support sustained $\mathrm{Cl}^{-}$and $\mathrm{HCO}_{3}{ }^{-}$secretion. Patients with diarrheal disorders, such as IBS-D, have been reported to have higher VIP concentrations within the gut $(140,141)$. Conversely, genetic ablation of VIP completely disrupts intestinal transit and basal secretory function in mice (142), mimicking Hirschsprung's Disease a congenital condition where newborns lack ENS innervation to the colon and rectum.

Post-synaptic VIP-ergic neurons also innervate submucosal blood vessels, as their nomenclature implies (143). The vasodilatory response can support secretion by supplying fluid to the epithelium, which will follow the osmotic gradient into the gut lumen if active anion secretion persists (144). This effect is likely mediated by VPAC1 receptors in the endothelium, although current supporting data have only been provided for extra-intestinal vessels (145). VPAC2 receptors are expressed in the smooth muscle itself (146), where release of VIP triggers muscle relaxation, contributing to the hyperemic response. Furthermore, VIP is found in inhibitory myenteric neurons where it plays a role in suppressing motility $(147,148)$. The combined effects of local VIP release culminate in the formation of watery stool, similar to that observed with cholinergic stimulation.

Often, cAMP and $\mathrm{Ca}^{2+}$ signaling (e.g., from M3R and VPAC1 receptors) work synergistically to produce a maximal secretory response $(149,150,151)$. There is significant 
overlap in these two signaling pathways and neither can proceed in true isolation. For example, some adenylate cyclases (which generate cAMP and lead to CFTR activation) are activated by the $\mathrm{Ca}^{2+}$-binding protein, calmodulin (152), which is a down-stream mediator of muscarinic signaling. Conversely, the inositol triphosphate receptor $\left(\mathrm{IP}_{3} \mathrm{R}\right)-$ in integral component of $\mathrm{Ca}^{2+}$ release from endoplasmic reticulum (ER) stores - is sensitized by cAMP (153), and $\mathrm{Ca}^{2+}$ transients in epithelia can be elicited by treatment with VIP (154). Even so-called $\mathrm{Ca}^{2+}$-activated $\mathrm{Cl}^{-}$channels (CaCCs), now identified as the TMEM16 gene family, have been reported to be dependent on the function of cAMP-regulated CFTR channels $(73,155)$, although these two pathways were once viewed as entirely independent. Aberrant cholinergic $\left(\mathrm{Ca}^{2+}\right.$-mediated) and VIP-ergic (cAMP-mediated) signaling have both been associated with ENS-driven pathologies, such as irritable bowel syndrome (IBS) $(13,156,157)$.

\section{Serotonin}

Serotonin (5-hydroxytryptamine; 5-HT) was first identified in the 1940s (158), but its influence over intestinal motility was documented even earlier, before the molecule itself was known (159). Although widely understood to be an important CNS neurotransmitter implicated in the control of mood, emotion and sleep, the vast majority ( $>95 \%)$ of all serotonin is housed within the gut (160). The name, "serotonin," itself is related to its vasoconstrictive properties, which results from platelets (the major carrier of serotonin in the blood) acquiring gut-derived serotonin as they pass through the GI circulation (161). The major source of serotonin production is the enterochromaffin cells (EC cells), a type of endocrine cell in the epithelium. Despite their scarcity, the overwhelming majority of serotonin production in the body is confined to this niche cell type. EC cells are electrically excitable and respond to mechanical distension by releasing serotonin through degranulation across their basolateral membranes $(162,163,164)$, where the "post- 
synaptic" intrinsic primary afferent terminals of myenteric neurons (as well as others) reside. This initiates a multi-step, reflexive secretomotor response necessary to secrete fluid and propel luminal contents forward (164). Cholinergic and VIP-ergic submucosal neurons are the end-effectors in the secretory reflex circuit $(164,165)$, while myenteric motor neurons simultaneously induce smooth muscle contraction (166). The ability of the gut to carry out this coordinated secretomotor reflex, independently of central innervation, is largely dependent on this phenomenon of serotonergic "synaptic" transmission from EC cells to the intrinsic afferents.

5- $\mathrm{HT}_{3}$ receptors on intrinsic afferent terminals in close proximity to EC cells $(162,167)$, as well as on post-synaptic terminals of efferent motor neurons (168), have the greatest involvement of the 5-HT receptor subtypes. Alosetron, a 5- $\mathrm{HT}_{3} \mathrm{R}$ antagonist, was a commonly used treatment for IBS-D because it interferes with aberrant serotonergic transmission from EC cells to afferent terminals, thus preventing unwanted peristalsis (i.e., frequent bowel movements) $(169,170)$. On the other hand, 5-HT receptor agonists, such as Tegasarod, can be affective in alleviating constipation, as they promote motility (171). Both of these drugs have declined in use however, due to deleterious side effects (169). Serotonin transporter (SERT) is expressed in epithelial cells and facilitates its uptake from the synaptic space (172), just as in the CNS. Selective serotonin reuptake inhibitors (SSRIs), a class of anti-depressant drugs (e.g., fluvoxamine), are consequently associated with a host of GI-related side effects (173).

Experiments using transgenic mice have revealed that serotonergic transmission is important primarily for motility (174). Deletion of tryptophan hydroxylase, the rate-limiting enzyme required for serotonin synthesis, in either EC cells (175) or ENS neurons (176) impairs peristalsis. Interestingly, while EC cell production of serotonin dwarfs that of ENS neurons themselves, it is the production of serotonin specifically within myenteric neurons that seems to 
be the most important for normal motive function (177). With respect to secretion, serotonin released from ENS neurons is not known to specifically act upon epithelial cells in the crypt or surface regions. Thus, it is not generally considered to be a classical "secretagogue." However, enterocytes in the crypt do express $5-\mathrm{HT}_{2}$ receptors that can be activated by the paracrine release of serotonin from nearby EC cells (168).

\subsubsection{Neuro-immune communication}

Many populations of immune cells reside within the GI tissue and are critical for host defense, as the lumen of the gut - and in particular, the colon - is home to innumerable microorganisms. Collectively, intestinal immune cells are referred to as gut-associated lymphoid tissue (GALT), and constitute over $75 \%$ of the body's total immune system (178). One of the important GALT components are Peyer's patches, concentrated foci of lymphocytes that form nodules just beneath the mucosa throughout the GI tract (179). These allow for a robust adaptive immune response to pathogenic substances, as T- and B-lymphocytes are very tightly packed in these structures, awaiting the signal to mobilize into action. Innervation of Peyer's patches by peptidergic (e.g., VIP and substance P) submucosal neurons has been known for some time (180). However, the extent to which the ENS interacts with resident immune populations - including not only lymphocytes in the Peyer's patches, but also resident macrophages and monocytes, $\mathrm{M}$ cells, circulating leukocytes, and others - is only recently beginning to emerge in its true scale.

Communication is now known to proceed both ways: neuron to immune effector cell and vice versa. For example, most circulating leukocytes express receptors for common neurotransmitters, such as acetylcholine and norepinephrine (originating from sympathetic nerve fibers) (181). Cholinergic signaling from the ENS, as well as the parasympathetic system, to these 
immune populations has a powerful immunosuppressive effect systemically and in the gut as well (7). Intestinal macrophages have also been shown to be heavily influenced by physical contact with myenteric or submucosal neurons, which differentially regulate their development into specific M2 or M1 phenotypes, respectively, through release of neurochemicals (182). As another example, Mast cells in the lamina propria are activated by certain neuropeptides (e.g., Substance P), and they subsequently degranulate to release large quantities of histamine (183). Mast cell number and degree of neurogenic activation are increased in patients with IBS-D (184). Histamine receptors are expressed in both enterocytes (185) - where their activation triggers $\mathrm{Cl}^{-}$secretion, and sensory afferents (186) - where they contribute to nociception and sensitization to other stimuli. Similarly, Substance P induces the production of pro-inflammatory cytokines (e.g., TNFFo, IL-6) from monocytes in the lamina propria (187), contributing to epithelial secretion as well.

Cytokines can also, in turn, potentiate neuronal firing and induce secretion and/or motility. This is believed to be central in the pathogenesis of many GI disorders like inflammatory bowel disease (IBD) and enterobacterial/enteroviral infection. Inflammation-induced changes to ENS activity can take the form of increased excitability. For example, IL-6 is dramatically upregulated in IBD and IBS patients, and potentiates the effects of other secretagogues in the submucosal plexus, contributing to enhanced secretory activity (188). In fact, application of plasma from IBD patients increased firing in rat submucosal neurons, but this was attenuated by neutralization of IL-6 and/or corticotrophin releasing factor (CRF) (189). In addition, anatomical changes to the ENS are associated with an inflammatory state as well. IL-1 $\beta$ and TNF- $\alpha$ have can activate neurite outgrowth via the NF-אB signaling pathway (190). Changes in ganglion density have also been associated with IBD as well (191). 
In summary, the ENS is a multifaceted and highly complex governing entity that controls both motility and secretion in the colon. Modulation of epithelial ion transport, specifically, by the ENS is multidimensional as well, as interplay between environmental, immunological and endocrine factors each have their own bearing on ENS-driven secretion (7). Many structural and functional aspects of ENS circuitry have been brought to light in recent years. Further exploration of the precise mechanisms of interaction between the ENS and effector cells in the epithelium, smooth muscle, and lymph tissue will give us insight into this relatively new platform from which to approach the treatment of diseases like IBS and IBD.

\section{2 - Endocrine Regulation of Colonic Electrolyte Transport}

Modulation of colonic ion transport by the ENS takes place on a short time scale. ENSdriven secretion across the epithelium occurs rapidly in response to various stimuli (discussed above). In addition, input from the ENS to the epithelium is generally reductive in terms of its influence on basal absorptive processes. That is to say that in the absence of input from the ENS, $\mathrm{Na}^{+}$and $\mathrm{Cl}^{-}$absorption is allowed to proceed unabated, and thus the release of ENS-derived neurotransmitters inhibits basal $\mathrm{Na}^{+} / \mathrm{Cl}^{-}$absorption and induces $\mathrm{Cl}$ and/or $\mathrm{HCO}_{3}^{-}$secretion simultaneously (Fig. 4). Most of these short-term alterations in ion transport are the result of transient changes in epithelial signaling pathways, such as receptor-induced increases in intracellular $\mathrm{Ca}^{2+}$ or cAMP. The activities of transporters and channel proteins can be rapidly adjusted in this context via changes in trafficking or phosphorylation. This allows for very dynamic, fine-tuned control over absorption and secretion.

However, long-term adjustments to epithelial transport are often mediated at a transcriptional level through endocrine mechanisms that either: 1) directly increase the production 
of transporters and channel proteins, or 2) indirectly regulate gene products that control the expression or trafficking of key transporter systems $(192,193)$. Furthermore, endocrine signals are the only external influences that can increase basal epithelial absorption, as neural and immune factors almost always induce a secretory response (103). For example, dehydration and pregnancy are both physiological conditions that require a sustained increase in colonic (and renal) $\mathrm{Na}^{+}$ absorption to maintain fluid volume in the body over relatively long time periods $(194,195)$. Endocrine regulation of epithelial transport is a crucial element in these situations because $\mathrm{Na}^{+}$ absorptive processes must be maintained above baseline levels over time. Hormonal factors bring about long-term changes necessary to maintain fluid and electrolyte homeostasis. Cortisol, aldosterone, somatostatin and the sex hormones estradiol and progesterone all play an important role in this process, as do many other endocrine signals.

\subsection{1 - Corticosteroid regulation of colonic ion transport.}

Corticosteroid hormones are produced in the adrenal cortex and have diverse biological functions. Among these are the regulation of metabolism and blood sugar levels, maintenance of fluid balance, adaptation to stress and suppression of inflammation (196). Two subsets of corticosteroid hormones - glucocorticoids and mineralocorticoids - are particularly important in regulating electrolyte transport in the intestine (192), as well as in the kidney $(197,198)$. Both of these are synthesized from cholesterol, although the activity of the enzymes required for glucocorticoid and mineralocorticoid synthesis are differentially expressed by specific cells in the adrenal cortex (199). Glucocorticoids are produced in the zona fasciculata via the $11 \beta$-hydroxylase enzyme, which converts the cholesterol-derived precursors into the active cortisol (200) (or corticosterone, in rodents). Exclusively expressed in the zona glomerulosa is the aldosterone 
synthase enzyme, which converts corticosterone into aldosterone. They activity of these various enzymes is also regulated by different biological signals as well.

Production of glucocorticoid hormones from the zona fasciculata cells of the adrenal cortex is mainly triggered by binding of circulating adrenal corticotropic hormone (ACTH) to its receptor on the plasma membrane (201). Upstream of this, ACTH produced by the anterior pituitary in response to corticotropin-releasing hormone $(\mathrm{CRH})$ sent from the hypothalamus. This pathway is a critical component to adaptation to stress by increasing circulating blood glucose levels and conferring behavioral and emotional changes in the CNS (202). The acute effects of a robust stress response generally inhibit absorption and associated blood flow (203), in lieu of diverting resources to brain and muscle to meet immediate demands. Under normal conditions however, basal glucocorticoid production is required for proper absorption, as described below.

Mineralocorticoid production is mainly triggered by the renin-angiotensin system in response to a reduction in circulating volume (i.e., hemorrhage or dehydration) via angiotensin-II receptors (204), or directly by an increase in extracellular $\mathrm{K}^{+}$(hyperkalemia) in zona glomerulosa cells (205). Glucocorticoids therefore regulate basal colonic transport in a normal, homeostatic manner, while mineralocorticoids become increasingly important in restoring fluid and electrolyte balance in response to significant perturbations to the system. While there is significant overlap in the end effects of GR and MR signaling with respect to colonic ion transport (206), some key differences to exist in terms of both their site of action and the specific transport pathways which the two hormones regulate.

The primary mechanism of action for corticosteroids (and any other steroid hormone) is different from that of the neurotransmitters, paracrine factors and cytokines described earlier. 
Glucocorticoid and mineralocorticoid receptors (GR and MR, respectively) are located within the cytosol of epithelial cells (207), as opposed to the plasma membrane receptors for neurotransmitters and cytokines. These hormones typically exert their effects through translocation to the nucleus upon binding to their hormone ligand, and they subsequently initiate the transcription of specific gene products (207). Some non-genomic actions have also been described as well (see below). Although synthetic corticosteroids are widely used in the clinic (208), including in the treatment of inflammatory bowel disease (209), the main biologically active, endogenous glucocorticoid and mineralocorticoid hormones are cortisol and aldosterone, respectively. Cortisol and aldosterone are similar in structure and closely related in the steroid synthesis pathways (199). However, cortisol has more widespread effects in the body, as almost every somatic tissue expresses the glucocorticoid receptor (210). On the other hand, mineralocorticoid receptors are present mainly within salt-transporting epithelia, including the distal colon. Circulating levels of cortisol are typically much higher than aldosterone - on the order of 100 -fold or more.

The distal nephron of the kidney, along with the distal colon constitute the main aldosterone-sensitive tissues, and both constitutively express MR (211). However, glucocorticoids can also bind to MR with similar affinity compared to GR. Because of this, and also because glucocorticoids are so much more abundant in the circulation, an enzyme called $11 \beta$ hydroxysteroid dehydrogenase type $2(11 \beta-H S D 2)$ is required for aldosterone sensitivity and is specifically expressed in these tissues $(211,212)$. 11ß-HSD 2 works by converting cortisol into the inactive cortisone, thus enabling the less abundant aldosterone to bind to MR and exert its effects. In this way, small changes in circulating aldosterone levels can have a substantial impact on MRregulated distal colonic electrolyte transport. Mutations in 11ß-HSD 2 that render the enzyme non- 
functional cause a form of juvenile hypertension called the "syndrome of apparent mineralocorticoid excess," due to increased occupancy of MR by cortisol, which cannot be eliminated in aldosterone-sensitive tissues (213). Excessive salt and water absorption ensues, causing hypervolemic hypertension.

Electroneutral $\mathrm{NaCl}$ absorption is stimulated by corticosteroid signaling in the proximal colon

In humans and rodents, infusion of corticosteroid hormones including cortisol (or corticosterone in rodents) and aldosterone, as well as synthetic compounds, transcriptionally upregulates the expression of electroneutral transporters in the proximal colon to allow for increased capacity for $\mathrm{Na}^{+}$and $\mathrm{Cl}^{-}$absorption $(206,214,215)$. In particular, NHE-3 protein abundance is enhanced in animals infused with mineralocorticoids over a period of hours to days (216). Low dose glucocorticoids produce a similar effect in the small intestine (217). Mineralocorticoid response elements (MREs) and glucocorticoid response elements (GREs) are found in the promoter region of NHE-3, where steroid-bound receptors can dock and initiate transcription $(218,219)$. As it turns out, corticosteroids were used in the treatment of IBD initially for their antiinflammatory effects (220), resulting from suppression of pro-inflammatory genes which are regulated by GREs in T-cells. Later, after subsequent investigation into the mechanism of action, it was discovered that intestinal $\mathrm{NaCl}$ absorption was also dramatically impacted by corticosteroid use, contributing to symptom alleviation as stool water content is reduced (221).

Basal circulating glucocorticoid levels are necessary for development of salt-transporting epithelia, including in the intestine. In healthy adults, basal electroneutral $\mathrm{NaCl}$ (NHE-3 dependent) absorption in the small and large intestine is also driven by constitutive glucocorticoid production (222). Adrenalectomized animals, which are unable to synthesize corticosteroids, have 
severely impaired absorptive capacity $(223,224)$. Replenishment of exogenous glucocorticoids is sufficient to restore normal absorptive function in adrenalectomized animals (225). Although mineralocorticoid (aldosterone) production is also eliminated in these animals, evidence suggests that mineralocorticoid activity is not essential for electroneutral absorption in the proximal colon, nor the small intestine (225). On the other hand, electrogenic (ENaC-mediated) absorption in the distal colon is critically dependent on mineralocorticoid signaling.

The rate of $\mathrm{Cl}^{-}$absorption in the proximal colon also increases in response to corticosteroids, though the net flux of $\mathrm{Na}^{+}$remains greater than that of $\mathrm{Cl}^{-}(62,215)$. In addition, expression of electroneutral $\mathrm{Cl}^{-} / \mathrm{HCO}_{3}{ }^{-}$exchangers is not increased in parallel to NHE-3 (226), suggesting the enhanced $\mathrm{Cl}^{-}$absorption occurs secondarily to NHE activity. Efflux of $\mathrm{Cl}^{-}$across the apical membrane, or inability of $\mathrm{Cl}^{-}$to transit the basolateral membrane as readily as $\mathrm{Na}^{+}$may be responsible for this discrepancy in the rates of flux for the two ion species. There may also be some involvement of apical $\mathrm{Cl}^{-} /$butyrate or $\mathrm{HCO}_{3}{ }^{-}$/butyrate exchangers as well, although this possibility has not been explored. It is not known whether SCFA transporters are transcriptionally regulated by MR or GR signaling. Rates of $\mathrm{Na}^{+}$and $\mathrm{Cl}^{-}$uptake are elevated by both butyrate and corticosteroids, independently. However, the effect of corticosteroids on butyrate uptake (which is now known to be closely tied to $\mathrm{Na}^{+}$uptake) has not been studied. If $\mathrm{Na}^{+}$uptake is more tightly coupled to butyrate uptake (as opposed to $\mathrm{Cl}^{-}$) under conditions of high MR/GR activity, this may explain the difference in corticosteroid-induced $\mathrm{Na}^{+}$versus $\mathrm{Cl}^{-}$absorption in the proximal colon. Interestingly, butyrate has transcriptional effects as well because it acts as a histone deacetylase inhibitor (49). In fact, there is some evidence to suggest that butyrate is necessary to confer aldosterone sensitivity in the colon $(227,228)$, raising the possibility of a powerful combined effect of the two. 
$\mathrm{Na}^{+} / \mathrm{K}^{+}$ATPase activity drives the overall absorptive process, as described in earlier sections. $\mathrm{Na}^{+} / \mathrm{K}^{+}$ATPase activity in the colon is also regulated by corticosteroids $(86,229)$, although there is some evidence that aldosterone controls $\mathrm{Na}^{+} / \mathrm{K}^{+}$ATPase expression indirectly through an increase in transporter density in the basolateral membrane rather than through direct transcriptional control $(85,230)$. This may be partially attributable to transcriptional regulation of non-transporter/channel gene targets of MR signaling. Among the most well-described of these are the serum- and glucocorticoid-regulated kinase 1 (SGK-1) $(231,232)$ and corticosteroid hormone inducing factor (CHIF) $(233,234)$, both of which are necessary for corticosteroid hormones to produce their full effects in transporting epithelial tissues, including the colon.

Electrogenic $\mathrm{Na}^{+}$absorption is stimulated and electroneutral $\mathrm{NaCl}$ absorption in inhibited by mineralocorticoid signaling in the distal colon.

As mentioned before, $\mathrm{ENaC}$ facilitates electrogenic $\mathrm{Na}^{+}$absorption and is found only in the distal segment of the colon. $\mathrm{ENaC}$ is comprised of three highly homologous subunits $(\alpha, \beta$ and $\gamma)$, two of which $(\beta$ and $\gamma$ ) are under direct transcriptional control by aldosterone $(235,236,237,238)$. ENaC degradation via the proteasome pathway is also inhibited by aldosterone through activation of SGK-1 (239). Apical ENaC-mediated $\mathrm{Na}^{+}$absorption is driven by basolateral $\mathrm{Na}^{+} / \mathrm{K}^{+}$ATPase activity, just as electroneutral absorption is in the proximal colon. In animals infused with aldosterone, or in animals with diet-induced secondary hyperaldosteronism, nearly all $\mathrm{Na}^{+}$absorption is inhibited by low-dose amiloride and is therefore dependent on $\mathrm{ENaC}$ $(235,240)$. At the same time, NHE-3 expression is inhibited by aldosterone (241). Effects on $\mathrm{Cl}^{-}$ $/ \mathrm{HCO}_{3}{ }^{-}$or other anion exchangers are not currently known. However, given that anion exchangers are driven by $\mathrm{Na}^{+} / \mathrm{H}^{+}$exchange, and that ${ }^{36} \mathrm{Cl}^{-}$fluxes are reduced by aldosterone (27), the 
functionality of anion exchangers in the distal colon is down-regulated by MR signaling, regardless of any potential effects on protein expression directly. Importantly, aldosterone-induced inhibition of electroneutral $\mathrm{NaCl}$ absorption, as well as stimulation of electrogenic $\mathrm{Na}^{+}$absorption, is prevented by treatment with the MR antagonist, spironolactone $(242,243)$. As such, MR-regulated $\mathrm{Na}^{+}$absorption is entirely electrogenic and electroneutral $\mathrm{NaCl}$ absorption completely suppressed.

Mineralocorticoid, but not glucocorticoid signaling induces $K^{+}$secretion in the proximal and distal colon.

The production of aldosterone is typically associated with the renin-angiotensin system, which is activated after a loss in blood volume (244). In this context, aldosterone serves to enhance $\mathrm{Na}^{+}$and water absorption to minimize fluid loss and restore balance. The other major trigger for aldosterone production is a rise in extracellular $\mathrm{K}^{+}\left[\mathrm{K}^{+}\right]_{\mathrm{o}}$, known as hyperkalemia. Even minute changes in $\left[\mathrm{K}^{+}\right]_{0}$ can be life-threatening because of the depolarizing effect on important electrically-excitable cell populations (i.e., neurons in the hypothalamus and brain stem). Zona glomerulosa cells in the adrenal cortex are depolarized by an increase in $\left[\mathrm{K}^{+}\right]_{\mathrm{o}}$, directly leading to $\mathrm{Ca}^{2+}$ influx that triggers a signaling cascade resulting in aldosterone synthesis (245). This mechanism bypasses the renin-angiotensin system, which also ultimately cause zona glomerulosa cell depolarization indirectly through binding of angiotensin II to receptors and downstream inhibition of plasma membrane $\mathrm{K}^{+}$channels (245).

Colonic $\mathrm{K}^{+}$transport is mediated by apical membrane large conductance $\mathrm{K}^{+}$(BK) $(97,246)$ and possibly intermediate conductance $\mathrm{K}^{+}$(IK) (94) channels, and aldosterone induces the expression of BK and IK (94) channels in the colon. Under normal conditions, there is a small amount of $\mathrm{K}^{+}$secretion in the proximal colon, but $\mathrm{K}^{+}$is absorbed in the distal colon by apical 
membrane $\mathrm{H}^{+} / \mathrm{K}^{+}$ATPases $(83,88)$. In the context of elevated MR signaling, net $\mathrm{K}^{+}$secretion is enhanced in the proximal colon and induced in the distal colon, primarily through an iberiotoxin (i.e., BK channel-dependent) mechanism $(92,95,246)$. Colonic $\mathrm{K}^{+}$secretion that is regulated by aldosterone is an imperative outlet mechanism in patients with end-stage renal disease (ESRD) $(100,101)$, who cannot adequately eliminate excessive $\mathrm{K}^{+}$through the kidney. Thorough characterization of the supporting elements of sustained $\mathrm{K}^{+}$secretion in response to aldosterone, such as accessory BK channel subunits $(\beta / \gamma)$ and basolateral $\mathrm{K}^{+}$loaders (i.e., $\mathrm{Na}^{+} / \mathrm{K}^{+}$ATPase versus NKCC1) is not yet complete.

Non-genomic effects of aldosterone on colonic ion transport.

Steroid hormones are now recognized to exert rapid effects on cellular activity which are not dependent on transcription. Often these effects are in line with transcriptional outcomes but require much less time to develop. This holds true for modulation of colonic epithelial transport by aldosterone as well (247), which produces a rapid induction of $\mathrm{Na}^{+}$absorptive and $\mathrm{K}^{+}$secretory process dependent one or more of the protein kinase C (PKC) isoforms $(248,249,250)$. At the same time, aldosterone also inhibits basolateral IK channel currents necessary for supporting $\mathrm{Cl}^{-}$ secretion in the crypts (251). Characterization of the non-genomic actions of aldosterone is an emerging and rapidly evolving field. Many aspects of rapid corticosteroid effects have yet to be explored.

\subsection{2 - Regulation of colonic ion transport by somatostatin.}

Somatostatin (SS) is a 14- or 28-animo acid peptide hormone produced in the pancreas, hypothalamus, or in the intestinal mucosa (252). The effects of SS are generally considered to be inhibitory, preventing the release of growth hormone from the pituitary and glucagon from the 
pancreas. The GI tract is no exception, where treatment with exogenous SS inhibits basal and agonist-induced $\mathrm{Cl}^{-}$secretion $(253,254)$. The somatostatin receptor is coupled to $\mathrm{G}_{\mathrm{c}}$, which inhibits the production of cAMP upon receptor binding. The inhibitory effects of SS with respect to epithelial secretion are thought to be dependent on the prevention of cAMP-dependent $\left[\mathrm{Ca}^{2+}\right]_{\mathrm{i}}$ increase, thus attenuating the stimulatory effect of other secretagogues $(255,256)$. BK-mediated $\mathrm{K}^{+}$secretion is also attenuated by SS in colonic epithelial cells (257). SS is one of the only known non-steroid agents that enhances basal absorption. Somatostatin analogs (SSAs) are used clinically for the treatment of a variety of endocrine disorders and conditions related to neo-endocrine tumors (258). However, certain GI-related conditions, such as refractory diarrhea and some post-surgical syndromes can be relieved by treatment with SSAs (258).

\subsection{3 - Regulation of colonic ion transport by sex steroids.}

Recent attention to sex as a biological variable has led to many discoveries related to sex differences across all disciplines of biomedical research. Epithelial transport in many tissues, including the colon, is governed by sex steroids in addition to the corticosteroids discussed previously (259). Sex steroid hormones usually exert their effects in the same way - by binding to intracellular receptors and causing their translocation to the nucleus to initiate the transcription of gene products. Evidence suggests that some sex steroids, especially $17-\beta$ estradiol $\left(\mathrm{E}_{2}\right)$, modulate epithelial growth (260) and tight junction barrier development (261), and may even have antitumorigenic properties (262) that are all mediated by transcriptional mechanisms. However, many of the documented effects on epithelial ion transport seem to occur through non-genomic effects $(263,264,265)$. There is some speculation that the steroid molecule itself can interact directly with target proteins to produce these effects (266). Recently, a membrane-bound estrogen receptor (ER) 
variant was also identified that probably mediates some non-transcriptional events via modulation of kinase-dependent signaling cascades (267).

Administration of exogenous $\mathrm{E}_{2}$ (estradiol) to isolated colonic mucosa inhibits both basal and cAMP-stimulated $\mathrm{Cl}^{-}$secretion through acute internalization of the basolateral $\mathrm{K}_{\mathrm{V}} 7.1$ channel (268), whose activity is critical for sustained $\mathrm{Cl}^{-}$efflux (see section 1.2.4). This was found to be dependent on the activity of PKC, similar to studies involving the corticosteroids, while inhibition of ER signaling via Tamoxifen did not interfere with the response. $E_{2}$ also dampened the secretory response to $V$. cholera and $E$. coli-derived enterotoxins in vitro through a similar mechanism (269). There is only very limited evidence to suggest a similar effect of progesterone, with a very mild effect seen in cultured epithelial cells with respect to cAMP-induced secretion (270). Meanwhile, the predominant male sex steroid, testosterone, has not been shown to produce any alterations to colonic electrolyte transport. Although $\mathrm{E}_{2}$ clearly influences epithelial growth and secretion via genomic and non-genomic mechanisms, respectively, the exact biological importance of these effects is unknown.

\section{Part 3: Specific Aims.}

As reviewed above, the regulation of ion transport in the intestine is a complex, but essential element of overall health. Many pathological conditions are either 1) the direct result of, 2) exacerbated by, or 3) ameliorated by changes to electrolyte transport in the colon. The focus of our recent studies has been centered around two areas in which the current understanding of colonic electrolyte transport regulation is lacking. The first involves identifying and characterizing a novel target, voltage-gated $\mathrm{K}_{\mathrm{V}} 7$ channels, in the neurogenic control of epithelial transport. The 
other is related to deepening our knowledge of the changes in epithelial transport mediated by mineralocorticoids (aldosterone), including investigation into $\mathrm{K}^{+}$and $\mathrm{Cl}^{-}$secretory pathways.

\section{1 - Specific Aim 1A: Determine the effect of neuronal $K_{V} 7$ (KCNQ) channel activation on} epithelial $\mathrm{Cl}^{-}$secretion. The enteric nervous system is known to regulate epithelial ion transport mainly by providing stimulatory (pro-secretion) input to epithelial cells. The $K C N Q$ family of voltage-gated $\left(\mathrm{K}_{\mathrm{V}} 7\right) \mathrm{K}^{+}$channels are a neuron-localized group of channels that hyperpolarize the plasma membrane of neurons and reduce excitability. $\mathrm{K}_{\mathrm{V}} 7$ channel activators have been used clinically to treat other conditions related to excessive neuronal firing (i.e., epilepsy or chronic pain), but the effects on ENS-driven secretion are not known. Using $\mathrm{I}_{\mathrm{SC}}$ recording techniques, pharmacological approaches and immunofluorescence microscopy, we tested the hypothesis that activation of ENS-localized $\mathrm{K}_{\mathrm{V}} 7$ channels suppresses epithelial $\mathrm{Cl}^{-}$secretion.

\section{2 - Specific Aim 1B: Determine the effect of neuronal $K_{V} 7$ (KCNQ) channel activation on}

epithelial $\mathrm{Na}^{+}$absorption. Stimulatory ENS input to the colonic epithelium is characterized by the additive effects of $\mathrm{Cl}^{-}$secretion and simultaneous inhibition of $\mathrm{Na}^{+}$absorption. This often produces a robust secretory response to physiological or pathological stimuli. Electroneutral $\mathrm{Na}^{+}$absorption is known to be inhibited by various secretagogues originating from secretomotor ENS neurons. ISC $_{\text {S }}$ and ${ }^{22} \mathrm{Na}^{+}$radioisotope flux experiments were performed to assess the effect of neuronal $\mathrm{K}_{\mathrm{V}} 7$ channel activation on epithelial $\mathrm{Na}^{+}$transport, with the prediction that reduced ENS input via a $\mathrm{K}_{\mathrm{V}} 7$ channel-activating drug would enhance basal $\mathrm{Na}^{+}$absorption in parallel to any suppressive effects on $\mathrm{Cl}^{-}$secretion. 
aldosterone-induced $\mathrm{K}^{+}$secretion in the distal colon. Although stimulation of $\mathrm{K}^{+}$secretion in the distal colon by aldosterone has been well-described, the role of $\mathrm{NKCC} 1$ in supporting $\mathrm{K}^{+}$secretion is not fully understood - in particular, transcriptional regulation of NKCC1 by aldosterone has not been established in the colon. Furthermore, it is not known whether changes in NKCC1 expression also influence $\mathrm{Cl}^{-}$secretion in parallel to $\mathrm{K}^{+}$secretion. Electrophysiological and molecular biological techniques were used to test the hypothesis that aldosterone transcriptionally upregulates $\mathrm{NKCC} 1$ in the distal colon, specifically to support $\mathrm{K}^{+}$secretion without concurrent enhancement of $\mathrm{Cl}^{-}$secretion.

3.4 - Specific Aim 2B: Determine whether aldosterone regulates the expression of critical components to electrogenic $\mathrm{K}^{+} / \mathrm{Cl}^{-}$secretion in the proximal colon. This work expands upon the existing literature which demonstrates that net basal $\mathrm{K}^{+}$and $\mathrm{Cl}^{-}$transport is differentially regulated by aldosterone in the proximal versus distal colon. Evidence of transcriptional regulation has not been provided, nor has a thorough assessment of agonist-stimulated changes in $\mathrm{Cl}^{-}$transport. We hypothesized that aldosterone reduces the capacity for $\mathrm{Cl}^{-}$secretion, likely via transcriptional effects on $\mathrm{Cl}^{-}$channel proteins and/or other components to the canonical secretory pathway. At the same time, $\mathrm{K}^{+}$secretion is known to be enhanced by aldosterone; thus, we predict the pathway mediating this is similar to the distal colon (i.e., upregulation of apical membrane BK and/or IK channels). Short-circuit current $\left(\mathrm{I}_{\mathrm{SC}}\right)$ electrophysiology and molecular biological approaches were used to assess the effect of aldosterone on basal and agonist $\left(\mathrm{Ca}^{2+/} \mathrm{cAMP}\right)$-stimulated $\mathrm{Cl}^{-}$and $\mathrm{K}^{+}$ secretion and expression of critical molecular components (i.e., CFTR, NKCC1, BK and IK channels). 


\section{REFERENCES}

1. Kunzelmann K, Mall M. Electrolyte transport in the mammalian colon: mechanisms and implications for disease. Physiol Rev. 2002;82(1):245-289.

doi:10.1152/physrev.00026.2001

2. Archampong EQ, Harris J, Clark CG. The absorption and secretion of water and electrolytes across the healthy and the diseased human colonic mucosa measured in vitro. Gut. 1972;13(11):880-886. doi:10.1136/gut.13.11.880

3. Kiela PR, Ghishan FK. Physiology of intestinal absorption and secretion. Best Pract Res Clin Gastroenterol. 2016;30(2):145-159. doi:10.1016/j.bpg.2016.02.007

4. Bitar KN. Function of gastrointestinal smooth muscle: from signaling to contractile proteins. Am J Med. 2003;115 Suppl:15S-23S. doi:10.1016/s0002-9343(03)00189-x

5. Granger DN, Holm L, Kvietys P. The Gastrointestinal Circulation: Physiology and Pathophysiology. Compr Physiol. 2015;5(3):1541-1583. doi:10.1002/cphy.c150007

6. Furness JB. The enteric nervous system and neurogastroenterology. Nat Rev Gastroenterol Hepatol. 2012;9(5):286-294. doi:10.1038/nrgastro.2012.32

7. Yoo B, Mazmanian S. The Enteric Network: Interactions between the Immune and Nervous Systems of the Gut. Immunity. 2017;46(6):910-926. doi:10.1016/j.immuni.2017.05.011. The

8. Cani PD. Interactions between gut microbes and host cells control gut barrier and metabolism. Int J Obes Suppl. 2016;6(Suppl 1):S28-S31. doi:10.1038/ijosup.2016.6

9. Nishida A, Inoue R, Inatomi O, Bamba S, Naito Y, Andoh A. Gut microbiota in the pathogenesis of inflammatory bowel disease. Clin J Gastroenterol. 2018;11(1):1-10. doi:10.1007/s12328-017-0813-5

10. Quigley EMM. Microbiota-Brain-Gut Axis and Neurodegenerative Diseases. Curr Neurol Neurosci Rep. 2017;17(12):94. doi:10.1007/s11910-017-0802-6

11. Rao MC. Physiology of Electrolyte Transport in the Gut: Implications for Disease. Compr Physiol. 2019;9(3):947-1023. doi:10.1002/cphy.c180011

12. Field M. Intestinal ion transport and the pathophysiology of diarrhea. J Clin Invest. 2003;111(7):931-943. doi:10.1172/JCI200318326

13. Mearin F, Lacy BE, Chang L, et al. Bowel Disorders. Gastroenterology. February 2016. doi:10.1053/j.gastro.2016.02.031

14. Smyth DH. Water movement across the mammalian gut. Symp Soc Exp Biol. $1965 ; 19: 307-328$. 
15. Hammel HT, Schlegel WM. Osmosis and solute-solvent drag: fluid transport and fluid exchange in animals and plants. Cell Biochem Biophys. 2005;42(3):277-345.

doi:10.1385/CBB:42:3:277

16. Spiller RC. Intestinal absorptive function. Gut. 1994;35(1 SUPPL.):5-9. doi:10.1136/gut.35.1_Suppl.S5

17. Noah TK, Donahue B, Shroyer NF. Intestinal development and differentiation. Exp Cell Res. 2011;317(19):2702-2710. doi:10.1016/j.yexcr.2011.09.006

18. van der Flier LG, Clevers H. Stem cells, self-renewal, and differentiation in the intestinal epithelium. Annu Rev Physiol. 2009;71:241-260.

doi:10.1146/annurev.physiol.010908.163145

19. Suzuki T. Regulation of intestinal epithelial permeability by tight junctions. Cell Mol Life Sci. 2013;70(4):631-659. doi:10.1007/s00018-012-1070-x

20. Capaldo CT, Powell DN, Kalman D. Layered defense: how mucus and tight junctions seal the intestinal barrier. J Mol Med (Berl). 2017;95(9):927-934. doi:10.1007/s00109-017$1557-\mathrm{x}$

21. Heinemann U, Schuetz A. Structural Features of Tight-Junction Proteins. Int J Mol Sci. 2019;20(23). doi:10.3390/ijms20236020

22. Laforenza U. Water channel proteins in the gastrointestinal tract. Mol Aspects Med. 2012;33(5-6):642-650. doi:10.1016/j.mam.2012.03.001

23. Ma T, Verkman AS. Aquaporin water channels in gastrointestinal physiology. J Physiol. 1999;517 ( Pt 2(Pt 2):317-326. doi:10.1111/j.1469-7793.1999.0317t.x

24. Matsuzaki T, Tajika Y, Ablimit A, Aoki T, Hagiwara H, Takata K. Aquaporins in the digestive system. Med electron Microsc Off J Clin Electron Microsc Soc Japan. 2004;37(2):71-80. doi:10.1007/s00795-004-0246-3

25. Clarke LL. A guide to Ussing chamber studies of mouse intestine. AJP Gastrointest Liver Physiol. 2009;296(6):G1151-G1166. doi:10.1152/ajpgi.90649.2008

26. Binder HJ, Rawlins L. Electrolyte transport across isolated large intestinal mucosa. Am J Physiol. 1973;225(5):1232-1239.

27. Foster ES, Zimmerman TW, Hayslett JP, Binder HJ. Corticosteroid alteration of active electrolyte transport in rat distal colon. Am J Physiol - Gastrointest Liver Physiol. 1983;8(5):668-675. doi:10.1152/ajpgi.1983.245.5.g668

28. Foster ES, Dudeja PK, Brasitus TA. Na $+-\mathrm{H}+$ exchange in rat colonic brush-border membrane vesicles. Am J Physiol. 1986;250(6(part 1)):781-787.

29. Binder HJ, Foster ES, Budinger ME, Hayslett JP. Mechanism of electroneutral sodium 
chloride absorption in distal colon of the rat. Gastroenterology. 1987;93(3):449-455. doi:10.1016/0016-5085(87)90905-X

30. Hubel KA. Effect of luminal sodium concentration on bicarbonate absorption in rat jejunum. J Clin Invest. 1973;52(12):3172-3179. doi:10.1172/JCI107517

31. Nellans HN, Frizzell RA, Schultz SG. Brush-border processes and transepithelial Na and Cl transport by rabbit ileum. Am J Physiol. 1974;226(5):1131-1141. doi:10.1152/ajplegacy.1974.226.5.1131

32. Greger R. Physiology of renal sodium transport. Am J Med Sci. 2000;319(1):51-62. doi:10.1097/00000441-200001000-00005

33. Zachos NC, Tse M, Donowitz M. Molecular physiology of intestinal $\mathrm{Na}+/ \mathrm{H}+$ exchange. Anпи Rev Physiol. 2005;67:411-443. doi:10.1146/annurev.physiol.67.031103.153004

34. Walker NM, Simpson JE, Brazill JM, et al. Role of down-regulated in adenoma anion exchanger in $\mathrm{HCO} 3-$ secretion across murine duodenum. Gastroenterology. 2009;136(3):893-901. doi:10.1053/j.gastro.2008.11.016

35. Kato A, Romero MF. Regulation of electroneutral $\mathrm{NaCl}$ absorption by the small intestine. Annu Rev Physiol. 2011;73:261-281. doi:10.1146/annurev-physiol-012110-142244

36. Whittamore JM, Hatch M. The anion exchanger PAT-1 (Slc26a6) does not participate in oxalate or chloride transport by mouse large intestine. Pflugers Arch. 2021;473(1):95106. doi: $10.1007 / \mathrm{s} 00424-020-02495-\mathrm{x}$

37. Foster ES, Dudeja PK, Brasitus TA. Contribution of $\mathrm{Cl}(-)-\mathrm{OH}-$ exchange to electroneutral $\mathrm{NaCl}$ absorption in rat distal colon. Am J Physiol. 1990;258(2 Pt 1):G261-7. doi:10.1152/ajpgi.1990.258.2.G261

38. Rajendran VM, Binder $\mathrm{HJ}$. Cl-HCO3 and $\mathrm{Cl}-\mathrm{OH}$ exchanges mediate $\mathrm{Cl}$ uptake in apical membrane vesicles of rat distal colon. Am J Physiol - Gastrointest Liver Physiol. 1993;264(5 27-5). doi:10.1152/ajpgi.1993.264.5.g874

39. Xue J, Thomas L, Tahmasbi M, et al. An inducible intestinal epithelial cell-specific NHE3 knockout mouse model mimicking congenital sodium diarrhea. Clin Sci (Lond). 2020;134(8):941-953. doi:10.1042/CS20200065

40. Alper SL, Sharma AK. The SLC26 Gene Family of Anion Transporters and Channels. Mol Aspects Med. 2013;34(2-3):494-515. doi:10.1016/j.mam.2012.07.009.

41. Lamprecht $\mathrm{G}$, Heil A, Baisch $\mathrm{S}$, et al. The down regulated in adenoma (dra) gene product binds to the second PDZ domain of the NHE3 kinase A regulatory protein (E3KARP), potentially linking intestinal $\mathrm{Cl}-/ \mathrm{HCO} 3-$ exchange to $\mathrm{Na}+/ \mathrm{H}+$ exchange. Biochemistry. 2002;41(41):12336-12342. doi:10.1021/bi0259103

42. Bachmann O, Juric M, Seidler U, Manns MP, Yu H. Basolateral ion transporters involved 
in colonic epithelial electrolyte absorption, anion secretion and cellular homeostasis. Acta Physiol. 2011;201(1):33-46. doi:10.1111/j.1748-1716.2010.02153.x

43. Ikuma M, Geibel J, Binder HJ, Rajendran VM. Characterization of Cl-HCO3 exchange in basolateral membrane of rat distal colon. Am J Physiol - Cell Physiol. 2003;285(4 544):912-921. doi:10.1152/ajpcell.00396.2002

44. Sangan P, Brill SR, Sangan S, Forbush B, Binder HJ. Basolateral K-Cl cotransporter regulates colonic potassium absorption in potassium depletion. $J$ Biol Chem.

2000;275(40):30813-30816. doi:10.1074/jbc.M003931200

45. Rehman S, Narayanan K, Nickerson AJ, et al. Parallel intermediate conductance K+ and $\mathrm{Cl}$ - channel activity mediates electroneutral $\mathrm{K}+$ exit across basolateral membranes in rat distal colon. Am J Physiol - Gastrointest Liver Physiol. 2020;319(2):G142-G150. doi:10.1152/ajpgi.00011.2020

46. Wong JMW, de Souza R, Kendall CWC, Emam A, Jenkins DJA. Colonic health: fermentation and short chain fatty acids. J Clin Gastroenterol. 2006;40(3):235-243. doi:10.1097/00004836-200603000-00015

47. Cummings JH, Pomare EW, Branch WJ, Naylor CPE, Macfarlane GT. Short chain fatty acids in human large intestine, portal, hepatic and venous blood. Gut. 1987;28:1221-1227. doi:10.1136/gut.28.10.1221

48. Liu H, Wang J, He T, et al. Butyrate: A double-edged sword for health? Adv Nutr. 2018;9(1):21-29. doi:10.1093/advances/nmx009

49. Hamer HM, Jonkers D, Venema K, Vanhoutvin S, Troost FJ, Brummer RJ. The role of butyrate on colonic function. Aliment Pharmacol Ther. 2008;27(2):104-119. doi:10.1111/j.1365-2036.2007.03562.x

50. Sandle GI. Salt and water absorption in the human colon: A modern appraisal. Gut. 1998;43(2):294-299. doi:10.1136/gut.43.2.294

51. Binder HJ, Mehta P. Short-chain fatty acids stimulate active sodium and chloride absorption in vitro in the rat distal colon. Gastroenterology. 1989;96(4):989-996. doi:10.1016/0016-5085(89)91614-4

52. Gonçalves P, Martel F. Regulation of colonic epithelial butyrate transport: Focus on colorectal cancer. Porto Biomed J. 2016;1(3):83-91. doi:10.1016/j.pbj.2016.04.004

53. Paroder V, Spencer SR, Paroder M, et al. Na+/monocarboxylate transport (SMCT) protein expression correlates with survival in colon cancer: Molecular characterization of SMCT. Proc Natl Acad Sci U S A. 2006;103(19):7270-7275. doi:10.1073/pnas.0602365103

54. Thibault R, De Coppet P, Daly K, et al. Down-Regulation of the Monocarboxylate Transporter 1 Is Involved in Butyrate Deficiency During Intestinal Inflammation. Gastroenterology. 2007;133(6):1916-1927. doi:10.1053/j.gastro.2007.08.041 
55. Reynolds DA, Rajendran VM, Binder HJ. Bicarbonate-stimulated [14C]butyrate uptake in basolateral membrane vesicles of rat distal colon. Gastroenterology. 1993;105(3):725732. doi:10.1016/0016-5085(93)90889-k

56. Mascolo N, Rajendran VM, Binder HJ. Mechanism of short-chain fatty acid uptake by apical membrane vesicles of rat distal colon. Gastroenterology. 1991;101(2):331-338. doi:10.1016/0016-5085(91)90008-9

57. Subramanya SB, Rajendran VM, Srinivasan P, Nanda Kumar NS, Ramakrishna BS, Binder HJ. Differential regulation of cholera toxin-inhibited $\mathrm{Na}-\mathrm{H}$ exchange isoforms by butyrate in rat ileum. Am J Physiol Gastrointest Liver Physiol. 2007;293(4):G857-63. doi:10.1152/ajpgi.00462.2006

58. Rajendran VM, Nanda Kumar NS, Tse CM, Binder HJ. Na-H Exchanger Isoform-2 (NHE2) Mediates Butyrate-dependent Na+ Absorption in Dextran Sulfate Sodium (DSS)induced Colitis. J Biol Chem. 2015;290(42):25487-25496. doi:10.1074/jbc.M115.654277

59. Kleyman TR, Eaton DC. Regulating ENaC's gate. Am J Physiol - Cell Physiol. 2020;318(1):C150-C162. doi:10.1152/ajpcell.00418.2019

60. Bridges RJ, Cragoe EJ, Frizzell RA, Benos DJ. Inhibition of colonic Na+ transport by amiloride analogues. Am J Physiol - Cell Physiol. 1989;256(1). doi:10.1152/ajpcell.1989.256.1.c67

61. Binder HJ, Singh SK, Geibel JP, Rajendran VM. Novel transport properties of colonic crypt cells: fluid absorption and Cl-dependent Na-H exchange. Comp Biochem Physiol A Physiol. 1997;118(2):265-269. doi:10.1016/s0300-9629(97)00079-0

62. Foster ES, Budinger ME, Hayslett JP, Binder HJ. Ion transport in proximal colon of the rat. Sodium depletion stimulates neutral sodium chloride absorption. J Clin Invest. 1986;77(1):228-235. doi:10.1172/JCI112281

63. Ray EC, Chen J, Kelly TN, et al. Human epithelial Na+ channel missense variants identified in the GenSalt study alter channel activity. Am J Physiol Renal Physiol. 2016;311(5):F908-F914. doi:10.1152/ajprenal.00426.2016

64. Cummings JH. Absorption and secretion by the colon. Gut. 1975;16(4):323-329. doi:10.1016/S0016-5085(69)80101-0

65. Jakab RL, Collaco AM, Ameen NA. Characterization of CFTR High Expresser cells in the intestine. Am J Physiol - Gastrointest Liver Physiol. 2013;305(6):453-465. doi:10.1152/ajpgi.00094.2013

66. Jakab RL, Collaco AM, Ameen NA. Physiological relevance of cell-specific distribution patterns of CFTR, NKCC1, NBCe1, and NHE3 along the crypt-villus axis in the intestine. Am J Physiol - Gastrointest Liver Physiol. 2011;300(1):82-98.

doi:10.1152/ajpgi.00245.2010 
67. Kerem B, Rommens JM, Buchanan JA, et al. Identification of the cystic fibrosis gene: genetic analysis. Science. 1989;245(4922):1073-1080. doi:10.1126/science.2570460

68. McIntosh I, Cutting GR. Cystic fibrosis transmembrane conductance regulator and the etiology and pathogenesis of cystic fibrosis. FASEB J Off Publ Fed Am Soc Exp Biol. 1992;6(10):2775-2782. doi:10.1096/fasebj.6.10.1378801

69. Greger R. Role of Cftr in the Colon. AnnuRevPhysiol. 2000;62:467-491. doi:10.1146/annurev.physiol.62.1.467

70. Cuthbert AW, MacVinish LJ, Hickman ME, Ratcliff R, Colledge WH, Evans MJ. Iontransporting activity in the murine colonic epithelium of normal animals and animals with cystic fibrosis. Pflugers Arch. 1994;428(5-6):508-515. doi:10.1007/BF00374572

71. Lee B, Hong GS, Lee SH, et al. Anoctamin 1/TMEM16A controls intestinal Cl- secretion induced by carbachol and cholera toxin. Exp Mol Med. 2019;51(8). doi:10.1038/s12276019-0287-2

72. Benedetto R, Ousingsawat J, Wanitchakool P, et al. Epithelial Chloride Transport by CFTR Requires TMEM16A. Sci Rep. 2017;7(1):1-13. doi:10.1038/s41598-017-10910-0

73. Vega G, Guequén A, Johansson MEV, et al. Normal calcium-activated anion secretion in a mouse selectively lacking TMEM16A in intestinal epithelium. Front Physiol. 2019;10(JUN):1-15. doi:10.3389/fphys.2019.00694

74. Ma T, Thiagarajah JR, Yang H, et al. Thiazolidinone CFTR inhibitor identified by highthroughput screening blocks cholera toxin-induced intestinal fluid secretion. $J$ Clin Invest. 2002;110(11):1651-1658. doi:10.1172/jci16112

75. Kelly T, Buxbaum J. Gastrointestinal Manifestations of Cystic Fibrosis. Dig Dis Sci. 2015;60(7):1903-1913. doi:10.1007/s10620-015-3546-7

76. Bachmann $\mathrm{O}$, Wüchner K, Rossmann $\mathrm{H}$, et al. Expression and regulation of the $\mathrm{Na}+-\mathrm{K}+-$ 2Cl- contransporter NKCC1 in the normal and CFTR-deficient murine colon. $J$ Physiol. 2003;549(2):525-536. doi:10.1113/jphysiol.2002.030205

77. Halm DR. Secretory control of basolateral membrane potassium and chloride channels in colonic crypt cells. Adv Exp Med Biol. 2005;559:119-129. doi:10.1007/0-387-23752-6_11

78. Nielsen MS, Warth R, Bleich M, Weyand B, Greger R. The basolateral Ca2+-dependent K+ channel in rat colonic crypt cells. Pflugers Arch Eur J Physiol. 1998;435(2):267-272. doi: $10.1007 / \mathrm{s} 004240050511$

79. Kunzelmann K, Hübner M, Schreiber R, et al. Cloning and function of the rat colonic epithelial K+ channel KVLQT1. J Membr Biol. 2001;179(2):155-164. doi: $10.1007 / \mathrm{s} 002320010045$

80. Seidler U, Blumenstein I, Kretz A, et al. A functional CFTR protein is required for mouse 
intestinal cAMP-, cGMP- and CA2+-dependent HCO3- secretion. J Physiol. 1997;505(2):411-423. doi:10.1111/j.1469-7793.1997.411bb.x

81. Garcia MAS, Yang N, Quinton PM. Normal mouse intestinal mucus release requires cystic fibrosis transmembrane regulator-dependent bicarbonate secretion. J Clin Invest. 2009;119(9):2613-2622. doi:10.1172/JCI38662

82. Yu K, Lujan R, Marmorstein A, Gabriel S, Hartzell HC. Bestrophin-2 mediates bicarbonate transport by goblet cells in mouse colon. J Clin Invest. 2010;120(5):17221735. doi:10.1172/JCI41129

83. Rajendran VM, Sandle GI. Colonic Potassium Absorption and Secretion in Health and Disease. Compr Physiol. 2018;8(4):1513-1536. doi:10.1002/cphy.c170030

84. Sorensen M V., Matos JE, Praetorius HA, Leipziger J. Colonic potassium handling. Pflugers Arch Eur J Physiol. 2010;459(5):645-656. doi:10.1007/s00424-009-0781-9

85. Hayslett JP, Binder HJ. Mechanism of potassium adaptation. Am J Physiol - Ren Fluid Electrolyte Physiol. 1982;12(2). doi:10.1152/ajprenal.1982.243.2.f103

86. Hayslett JP, Myketey N, Binder HJ, Aronson PS. Mechanism of increased potassium secretion in potassium loading and sodium deprivation. Am J Physiol - Ren Fluid Electrolyte Physiol. 1980;8(4):378-382. doi:10.1152/ajprenal.1980.239.4.f378

87. Agarwal R, Afzalpurkar R, Fordtran JS. Pathophysiology of potassium absorption and secretion by the human intestine. Gastroenterology. 1994;107(2):548-571.

doi:10.1016/0016-5085(94)90184-8

88. Rajendran VM, Singh SK, Geibel J, Binder HJ. Differential localization of colonic H+$\mathrm{K}+$-ATPase isoforms in surface and crypt cells. Am J Physiol - Gastrointest Liver Physiol. 1998;274(2 37-2):424-429. doi:10.1152/ajpgi.1998.274.2.g424

89. Watanabe T, Suzuki T, Suzuki Y. Ouabain-sensitive K(+)-ATPase in epithelial cells from guinea pig distal colon. Am J Physiol. 1990;258(4 Pt 1):G506-11.

doi:10.1152/ajpgi.1990.258.4.G506

90. Grotjohann I, Gitter AH, Köckerling A, Bertog M, Schulzke JD, Fromm M. Localization of cAMP- and aldosterone-induced $\mathrm{K}+$ secretion in rat distal colon by conductance scanning. J Physiol. 1998;507(2):561-570. doi:10.1111/j.1469-7793.1998.561bt.x

91. Meneton P, Schultheis PJ, Greeb J, et al. Increased sensitivity to $\mathrm{K}+$ deprivation in colonic H,K-ATPase-deficient mice. J Clin Invest. 1998;101(3):536-542. doi:10.1172/JCI1720

92. Sausbier M, Matos JE, Sausbier U, et al. Distal colonic K+ secretion occurs via BK channels. J Am Soc Nephrol. 2006;17(5):1275-1282. doi:10.1681/ASN.2005101111

93. Zhang J, Halm ST, Halm DR. Role of the BK channel (KCa1.1) during activation of electrogenic $\mathrm{K}+$ secretion in guinea pig distal colon. Am J Physiol - Gastrointest Liver 
Physiol. 2012;303(12):1322-1334. doi:10.1152/ajpgi.00325.2012

94. Singh SK, O'Hara B, Talukder JR, Rajendran VM. Aldosterone induces active K+ secretion by enhancing mucosal expression of Kcnn4c and Kcnmal channels in rat distal colon. Am J Physiol - Cell Physiol. 2012;302(9):1353-1360.

doi:10.1152/ajpcell.00216.2011

95. Sørensen M V., Strandsby AB, Larsen CK, Praetorius HA, Leipziger J. The secretory KCa1.1 channel localises to crypts of distal mouse colon: Functional and molecular evidence. Pflugers Arch Eur J Physiol. 2011;462(5):745-752. doi:10.1007/s00424-011$1000-\mathrm{z}$

96. Sandle GI, Perry MD, Mathialahan T, et al. Altered cryptal expression of luminal potassium (BK) channels in ulcerative colitis. J Pathol. 2007;212(1):66-73. doi:10.1002/path.2159

97. Lomax B, Mcnicholas M, Lombes M, Sandle I, Mcnicholas M. Aldosterone-induced apical $\mathrm{Na}+$ and $\mathrm{K}+$ conductances are located primarily in surface cells in rat distal colon. 1994;29:G71-G82.

98. Halm ST, Liao T, Halm DR. Distinct $\mathrm{K}+$ conductive pathways are required for $\mathrm{Cl}-$ and $\mathrm{K}+$ secretion across distal colonic epithelium. Am J Physiol - Cell Physiol. 2006;291(4):636648. doi:10.1152/ajpcell.00557.2005

99. Hay-Schmidt A, Grunnet M, Abrahamse SL, Knaus HG, Klaerke DA. Localization of $\mathrm{Ca} 2+$-activated big-conductance $\mathrm{K}+$ channels in rabbit distal colon. Pflugers Arch Eur $J$ Physiol. 2003;446(1):61-68. doi:10.1007/s00424-002-0983-x

100. Mathialahan T, Maclennan KA, Sandle LN, Verbeke C, Sandle GI. Enhanced large intestinal potassium permeability in end-stage renal disease. J Pathol. 2005;206(1):46-51. doi:10.1002/path. 1750

101. Mathialahan T, Sandle GI. Dietary potassium and laxatives as regulators of colonic potassium secretion in end-stage renal disease. Nephrol Dial Transplant. 2003;18(2):341347. doi:10.1093/ndt/18.2.341

102. Van Dinter TG, Fuerst FC, Richardson CT, et al. Stimulated active potassium secretion in a patient with colonic pseudo-obstruction: A new mechanism of secretory diarrhea. Gastroenterology. 2005;129(4):1268-1273. doi:10.1053/j.gastro.2005.07.029

103. Montrose MH, Keely SJ, Barrett KE. Electrolyte secretion and absorption: Small intestine and colon. In: Yamada T, ed. Textbook of Gastroenterology. 4th ed. ; 2003:308-340. doi:https://doi.org/10.1002/9781444303254.ch14

104. Spencer NJ, Hu H. Enteric nervous system: sensory transduction, neural circuits and gastrointestinal motility. Nat Rev Gastroenterol Hepatol. 2020;17(6):338-351. doi:10.1038/s41575-020-0271-2 
105. Taylor GS, Bywater RA. Intrinsic control of the gut. Baillieres Clin Gastroenterol. 1988;2(1):1-22. doi:10.1016/0950-3528(88)90018-8

106. Brierley SM, Hibberd TJ, Spencer NJ. Spinal afferent innervation of the colon and rectum. Front Cell Neurosci. 2018;12(December). doi:10.3389/fncel.2018.00467

107. Hibberd TJ, Kestell GR, Kyloh MA, Brookes SJH, Wattchow DA, Spencer NJ. Identification of different functional types of spinal afferent neurons innervating the mouse large intestine using a novel CGRP $\alpha$ transgenic reporter mouse. Am J Physiol Gastrointest Liver Physiol. 2016;310(8):G561-73. doi:10.1152/ajpgi.00462.2015

108. Smith-Edwards KM, Najjar SA, Edwards BS, Howard MJ, Albers KM, Davis BM. Extrinsic Primary Afferent Neurons Link Visceral Pain to Colon Motility Through a Spinal Reflex in Mice. Gastroenterology. 2019;157(2):522-536.e2. doi:10.1053/j.gastro.2019.04.034

109. Lomax AE, Furness JB. Neurochemical classification of enteric neurons in the guinea-pig distal colon. Cell Tissue Res. 2000;302(1):59-72. doi:10.1007/s004410000260

110. Reed DE, Vanner SJ. Converging and diverging cholinergic inputs from submucosal neurons amplify activity of secretomotor neurons in guinea-pig ileal submucosa. Neuroscience. 2001;107(4):685-696. doi:10.1016/s0306-4522(01)00392-x

111. Reed DE, Vanner S. Mucosal stimulation activates secretomotor neurons via long myenteric pathways in guinea pig ileum. Am J Physiol Gastrointest Liver Physiol. 2007;292(2):G608-14. doi:10.1152/ajpgi.00364.2006

112. Berthoud HR, Carlson NR, Powley TL. Topography of efferent vagal innervation of the rat gastrointestinal tract. Am J Physiol - Regul Integr Comp Physiol. 1991;260(1 291):200-207. doi:10.1152/ajpregu.1991.260.1.r200

113. Neunlist M, Reichelt D, Michel K, Pfannkuche H, Hoppe S, Schemann M. The Enteric Nervous System: Region and Target Specific Projections and Neurochemical Codes. Eur J Morphol. 1999;37(4):233-240. doi:10.1076/ejom.37.4.233.4720

114. Foong JPP, Tough IR, Cox HM, Bornstein JC. Properties of cholinergic and noncholinergic submucosal neurons along the mouse colon. J Physiol. 2014;592(4):777-793. doi:10.1113/jphysiol.2013.265686

115. Mawe GM, Hoffman JM. Serotonin signalling in the gut-functions, dysfunctions and therapeutic targets. Nat Rev Gastroenterol Hepatol. 2013;10(8):473-486. doi:10.1038/nrgastro.2013.105

116. Bridges BYRJ, Rack M, Rummel W, Schreiner J, Saar DH. Mucosal Plexus and Electrolyte Transport Across the Rat Colonic Mucosa. J Physiol. 1986;376:531-542. doi:10.1113/jphysiol.1986.sp016168

117. Garza A, Huang LZ, Son J-H, Winzer-Serhan UH. Expression of nicotinic acetylcholine 
receptors and subunit messenger RNAs in the enteric nervous system of the neonatal rat. Neuroscience. 2009;158(4):1521-1529. doi:10.1016/j.neuroscience.2008.11.027

118. Gwynne RM, Bornstein JC. Synaptic transmission at functionally identified synapses in the enteric nervous system: roles for both ionotropic and metabotropic receptors. Curr Neuropharmacol. 2007;5(1):1-17. doi:10.2174/157015907780077141

119. Delbro DS, Lange S. Effect of ganglionic blocking compounds on in-vivo fluid secretion in the rat small intestine. J Pharm Pharmacol. 1997;49(11):1109-1113. doi:10.1111/j.2042-7158.1997.tb06051.x

120. Diener M, Knobloch SF, Bridges RJ, Keilmann T, Rummel W. Cholinergic-mediated secretion in the rat colon: neuronal and epithelial muscarinic responses. Eur J Pharmacol. 1989;168(2):219-229. doi:10.1016/0014-2999(89)90568-2

121. Lindqvist SM, Sharp P, Johnson IT, Satoh Y, Williams MR. Acetylcholine-induced calcium signaling along the rat colonic crypt axis. Gastroenterology. 1998;115(5):11311143. doi:10.1016/s0016-5085(98)70084-8

122. Haberberger R, Schultheiss G, Diener M. Epithelial muscarinic M1 receptors contribute to carbachol-induced ion secretion in mouse colon. Eur J Pharmacol. 2006;530(3):229-233. doi:10.1016/j.ejphar.2005.11.055

123. North RA, Surprenant A. Muscarinic Receptors on Neurones of the Submucous Plexus. Muscarinic Recept Subtypes GI Tract. 1985:28-32. doi:10.1007/978-3-642-70668-4_4

124. Schultheiss G, Diener M. K+ and Cl- conductances in the distal colon of the rat. Gen Pharmacol. 1998;31(3):337-342. doi:10.1016/s0306-3623(97)00458-8

125. Hennig B, Schultheiss G, Kunzelmann K, Diener M. Ca2+-induced Cl- efflux at rat distal colonic epithelium. J Membr Biol. 2008;221(2):61-72. doi:10.1007/s00232-007-9078-0

126. Reynolds A, Parris A, Evans LA, et al. Dynamic and differential regulation of NKCC1 by calcium and cAMP in the native human colonic epithelium. J Physiol. 2007;582(2):507524. doi:10.1113/jphysiol.2007.129718

127. Heinke B, Ribeiro R, Diener M. Involvement of calmodulin and protein kinase $\mathrm{C}$ in the regulation of $\mathrm{K}+$ transport by carbachol across the rat distal colon. Eur J Pharmacol. 1999;377(1):75-80. doi:10.1016/S0014-2999(99)00416-1

128. Flemmer AW, Giménez I, Dowd BFX, Darman RB, Forbush B. Activation of the Na-K$\mathrm{Cl}$ cotransporter NKCC1 detected with a phospho-specific antibody. J Biol Chem. 2002;277(40):37551-37558. doi:10.1074/jbc.M206294200

129. Halm DR, Halm ST. Secretagogue response of goblet cells and columnar cells in human colonic crypts. Am J Physiol Cell Physiol. 2000;278(1):C212-33.

doi:10.1152/ajpcell.2000.278.1.C212 
130. Phillips TE. Both crypt and villus intestinal goblet cells secrete mucin in response to cholinergic stimulation. Am J Physiol - Gastrointest Liver Physiol. 1992;262(2 25-2). doi:10.1152/ajpgi.1992.262.2.g327

131. Li X, Zhang $\mathrm{H}$, Cheong A, et al. Carbachol regulation of rabbit ileal brush border Na+-H+ exchanger 3 (NHE3) occurs through changes in NHE3 trafficking and complex formation and is Src dependent. J Physiol. 2004;556(Pt 3):791-804.

doi:10.1113/jphysiol.2004.060921

132. Zachos NC, van Rossum DB, Li X, et al. Phospholipase C- $\gamma$ binds directly to the $\mathrm{Na}+/ \mathrm{H}+$ exchanger 3 and is required for calcium regulation of exchange activity. J Biol Chem. 2009;284(29):19437-19444. doi:10.1074/jbc.M109.006098

133. Yang J, Singh V, Chen TE, et al. NHERF2/NHERF3 protein heterodimerization and macrocomplex formation are required for the inhibition of NHE3 activity by carbachol. $J$ Biol Chem. 2014;289(29):20039-20053. doi:10.1074/jbc.M114.562413

134. Lamprecht G, Gaco V, Turner JR, Natour D, Gregor M. Regulation of the intestinal anion exchanger dRA (Downregulated in Adenoma). Ann N Y Acad Sci. 2009;1165:261-266. doi:10.1111/j.1749-6632.2009.04044.x

135. Iwasaki M, Akiba Y, Kaunitz JD. Recent advances in vasoactive intestinal peptide physiology and pathophysiology: Focus on the gastrointestinal system [version 1; peer review: 4 approved]. F1000Research. 2019;8:1-13. doi:10.12688/f1000research.18039.1

136. Jayawardena D, Guzman G, Gill RK, Alrefai WA, Onyuksel H, Dudeja PK. Expression and localization of VPAC1, the major receptor of vasoactive intestinal peptide along the length of the intestine. Am J Physiol Gastrointest Liver Physiol. 2017;313(1):G16-G25. doi:10.1152/ajpgi.00081.2017

137. Seidler U, Blumenstein I, Kretz A, et al. A functional CFTR protein is required for mouse intestinal cAMP-, cGMP- and Ca(2+)-dependent HCO3- secretion. J Physiol. 1997;505 ( Pt 2(Pt 2):411-423. doi:10.1111/j.1469-7793.1997.411bb.x

138. Schwartz CJ, Kimberg D V, Sheerin HE, Field M, Said SI. Vasoactive intestinal peptide stimulation of adenylate cyclase and active electrolyte secretion in intestinal mucosa. $J$ Clin Invest. 1974;54(3):536-544. doi:10.1172/JCI107790

139. Izu LT, McCulle SL, Ferreri-Jacobia MT, Devor DC, Duffey ME. Vasoactive intestinal peptide-stimulated Cl- secretion: Activation of cAMP-dependent K+ channels. J Membr Biol. 2002;186(3):145-157. doi:10.1007/s00232-001-0145-7

140. Sohn W, Lee OY, Lee SP, et al. Mast cell number, substance P and vasoactive intestinal peptide in irritable bowel syndrome with diarrhea. Scand J Gastroenterol. 2014;49(1):4351. doi:10.3109/00365521.2013.857712

141. Palsson OS, Morteau O, Bozymski EM, et al. Elevated vasoactive intestinal peptide concentrations in patients with irritable bowel syndrome. Dig Dis Sci. 2004;49(7-8):1236- 
1243. doi:10.1023/b:ddas.0000037818.64577.ef

142. Lelievre V, Favrais G, Abad C, et al. Gastrointestinal dysfunction in mice with a targeted mutation in the gene encoding vasoactive intestinal polypeptide: A model for the study of intestinal ileus and Hirschsprung's disease. Peptides. 2007;28(9):1688-1699. doi:10.1016/j.peptides.2007.05.006

143. De Fontgalland D, Wattchow DA, Costa M, Brookes SJH. Immunohistochemical characterization of the innervation of human colonic mesenteric and submucosal blood vessels. Neurogastroenterol Motil. 2008;20(11):1212-1226. doi:10.1111/j.13652982.2008.01150.x

144. Eklund S, Jodal M, Lundgren O, Sjöqvist A. Effects of vasoactive intestinal polypeptide on blood flow, motility and fluid transport in the gastrointestinal tract of the cat. Acta Physiol Scand. 1979;105(4):461-468. doi:10.1111/j.1748-1716.1979.tb00111.x

145. Grant S, Lutz EM, McPhaden AR, Wadsworth RM. Location and function of VPAC1, VPAC2 and NPR-C receptors in VIP-induced vasodilation of porcine basilar arteries. $J$ Cereb blood flow Metab Off J Int Soc Cereb Blood Flow Metab. 2006;26(1):58-67. doi:10.1038/sj.jcbfm.9600163

146. Harmar AJ, Sheward WJ, Morrison CF, Waser B, Gugger M, Reubi JC. Distribution of the VPAC2 receptor in peripheral tissues of the mouse. Endocrinology. 2004;145(3):12031210. doi:10.1210/en.2003-1058

147. Furness JB, Costa M. Projections of intestinal neurons showing immunoreactivity for vasoactive intestinal polypeptide are consistent with these neurons being the enteric inhibitory neurons. Neurosci Lett. 1979;15(2-3):199-204. doi:10.1016/03043940(79)96113-5

148. Sang Q, Williamson S, Young HM. Projections of chemically identified myenteric neurons of the small and large intestine of the mouse. J Anat. 1997;190(2):209-222. doi:10.1017/S0021878296001598

149. Cartwright CA, McRoberts JA, Mandel KG, Dharmsathaphorn K. Synergistic action of cyclic adenosine monophosphate- and calcium-mediated chloride secretion in a colonic epithelial cell line. J Clin Invest. 1985;76(5):1837-1842. doi:10.1172/JCI112176

150. Vajanaphanich M, Schultz C, Tsien RY, Traynor-Kaplan AE, Pandol SJ, Barrett KE. Cross-talk between calcium and cAMP-dependent intracellular signaling pathways. Implications for synergistic secretion in T84 colonic epithelial cells and rat pancreatic acinar cells. J Clin Invest. 1995;96(1):386-393. doi:10.1172/JCI118046

151. Barrett KE, Keely SJ. Chloride secretion by the intestinal epithelium: molecular basis and regulatory aspects. Annu Rev Physiol. 2000;62:535-572.

doi:10.1146/annurev.physiol.62.1.535

152. Pinkus LM, Sulimovici S, Susser FI, Roginsky MS. Involvement of calmodulin in the 
regulation of adenylate cyclase activity in guinea-pig enterocytes. Biochim Biophys Acta. 1983;762(4):552-559. doi:10.1016/0167-4889(83)90059-9

153. Burgess GM, Bird GS, Obie JF, Putney JWJ. The mechanism for synergism between phospholipase C- and adenylylcyclase-linked hormones in liver. Cyclic AMP-dependent kinase augments inositol trisphosphate-mediated $\mathrm{Ca} 2+$ mobilization without increasing the cellular levels of inositol polyphosphates. J Biol Chem. 1991;266(8):4772-4781.

154. Hagen BM, Bayguinov O, Sanders KM. VIP and PACAP regulate localized Ca2+ transients via cAMP-dependent mechanism. Am J Physiol - Cell Physiol. 2006;291(2):375-385. doi:10.1152/ajpcell.00495.2005

155. Kunzelmann K, Centeio R, Wanitchakool P, et al. Control of Ion Transport by Tmem16a Expressed in Murine Intestine. Front Physiol. 2019;10(October):1-10. doi:10.3389/fphys.2019.01262

156. Camilleri M. Intestinal secretory mechanisms in irritable bowel syndrome-diarrhea. Clin Gastroenterol Hepatol Off Clin Pract J Am Gastroenterol Assoc. 2015;13(6):1051-1052. doi:10.1016/j.cgh.2014.07.020

157. Saha L. Irritable bowel syndrome: pathogenesis, diagnosis, treatment, and evidence-based medicine. World J Gastroenterol. 2014;20(22):6759-6773. doi:10.3748/wjg.v20.i22.6759

158. Rapport MM, Green AA, Page IH. Serum Vasoconstrictor (Serotonin). J Biol Chem. 1948;176(3):1243-1251. doi:10.1016/s0021-9258(18)57137-4

159. Erspamer V. Experimental research on the biological significance of enterochromaffin cells. Arch Fisiol. 1937;37:156-169.

160. Gershon MD. 5-Hydroxytryptamine (serotonin) in the gastrointestinal tract. Curr Opin Endocrinol Diabetes Obes. 2013;20(1):14-21. doi:10.1097/MED.0b013e32835bc703

161. HUGHES FB, BRODIE BB. The mechanism of serotonin and catecholamine uptake by platelets. J Pharmacol Exp Ther. 1959;127:96-102.

162. Bellono NW, Bayrer JR, Leitch DB, et al. Enterochromaffin Cells Are Gut Chemosensors that Couple to Sensory Neural Pathways. Cell. 2017;170(1):185-198.e16. doi:10.1016/j.cell.2017.05.034

163. Alcaino C, Knutson KR, Treichel AJ, et al. A population of gut epithelial enterochromaffin cells is mechanosensitive and requires Piezo2 to convert force into serotonin release. Proc Natl Acad Sci U S A. 2018;115(32):E7632-E7641. doi:10.1073/pnas.1804938115

164. Sidhu M, Cooke HJ. Role for 5-HT and ACh in submucosal reflexes mediating colonic secretion. Am J Physiol - Gastrointest Liver Physiol. 1995;269(3 32-3). doi:10.1152/ajpgi.1995.269.3.g346 
165. Hubel KA. Intestinal nerves and ion transport: stimuli, reflexes, and responses. Am J Physiol. 1985;248(3 Pt 1):G261-71. doi:10.1152/ajpgi.1985.248.3.G261

166. Grundy D. 5-HT system in the gut: roles in the regulation of visceral sensitivity and motor functions. Eur Rev Med Pharmacol Sci. 2008;12 Suppl 1:63-67.

167. Kunze WAA, Bornstein JC, Furness JB. Identification of sensory nerve cells in a peripheral organ (the intestine) of a mammal. Neuroscience. 1995;66(1):1-4. doi:10.1016/0306-4522(95)00067-S

168. Mawe GM, Hoffman JM. Serotonin signalling in the gut--functions, dysfunctions and therapeutic targets. Nat Rev Gastroenterol Hepatol. 2013;10(8):473-486.

doi:10.1038/nrgastro.2013.105

169. Cangemi DJ, Lacy BE. Management of irritable bowel syndrome with diarrhea: a review of nonpharmacological and pharmacological interventions. Therap Adv Gastroenterol. 2019;12:1756284819878950. doi:10.1177/1756284819878950

170. Houghton LA, Foster JM, Whorwell PJ. Alosetron, a 5-HT3 receptor antagonist, delays colonic transit in patients with irritable bowel syndrome and healthy volunteers. Aliment Pharmacol Ther. 2000;14(6):775-782. doi:10.1046/j.1365-2036.2000.00762.x

171. Madia VN, Messore A, Saccoliti F, et al. Tegaserod for the Treatment of Irritable Bowel Syndrome. Antiinflamm Antiallergy Agents Med Chem. 2020;19(4):342-369. doi:10.2174/1871523018666190911121306

172. Wade PR, Chen J, Jaffe B, Kassem IS, Blakely RD, Gershon MD. Localization and function of a 5-HT transporter in crypt epithelia of the gastrointestinal tract. J Neurosci. 1996;16(7):2352-2364. doi:10.1523/JNEUROSCI.16-07-02352.1996

173. Wagner W, Plekkenpol B, Gray TE, Vlaskamp H, Essers H. Review of fluvoxamine safety database. Drugs. 1992;43 Suppl 2:44-48. doi:10.2165/00003495-199200432-00008

174. Terry N, Margolis KG. Serotonergic Mechanisms Regulating the GI Tract: Experimental Evidence and Therapeutic Relevance. Handb Exp Pharmacol. 2017;239:319-342. doi:10.1007/164_2016_103

175. Heredia DJ, Gershon MD, Koh SD, Corrigan RD, Okamoto T, Smith TK. Important role of mucosal serotonin in colonic propulsion and peristaltic reflexes: In vitro analyses in mice lacking tryptophan hydroxylase 1. J Physiol. 2013;591(23):5939-5957. doi:10.1113/jphysiol.2013.256230

176. Neal KB, Parry LJ, Bornstein JC. Strain-specific genetics, anatomy and function of enteric neural serotonergic pathways in inbred mice. J Physiol. 2009;587(3):567-586. doi:10.1113/jphysiol.2008.160416

177. Spencer NJ, Nicholas SJ, Robinson L, et al. Mechanisms underlying distension-evoked peristalsis in guinea pig distal colon: Is there a role for enterochromaffin cells? Am J 
Physiol - Gastrointest Liver Physiol. 2011;301(3):519-527. doi:10.1152/ajpgi.00101.2011

178. Genton L, Kudsk KA. Interactions between the enteric nervous system and the immune system: Role of neuropeptides and nutrition. Am J Surg. 2003;186(3):253-258. doi:10.1016/S0002-9610(03)00210-1

179. Heel KA, McCauley RD, Papadimitriou JM, Hall JC. Review: Peyer's patches. $J$ Gastroenterol Hepatol. 1997;12(2):122-136. doi:10.1111/j.1440-1746.1997.tb00395.x

180. Vulchanova L, Casey MA, Crabb GW, Kennedy WR, Brown DR. Anatomical Evidence for Enteric Neuroimmune Interactions in Peyer's Patches. J Neuroimmunol. 2007;185(1):64-74. doi:10.1021/nn2045246.Multifunctional

181. Serafini N, Vosshenrich CAJ, Di Santo JP. Transcriptional regulation of innate lymphoid cell fate. Nat Rev Immunol. 2015;15(7):415-428. doi:10.1038/nri3855

182. Gabanyi I, Muller PA, Feighery L, Oliveira TY, Costa-Pinto FA, Mucida D. Neuroimmune Interactions Drive Tissue Programming in Intestinal Macrophages. Cell. 2016;164(3):378-391. doi:10.1016/j.cell.2015.12.023

183. Yu Y, Daly DM, Adam IJ, et al. Interplay between mast cells, enterochromaffin cells, and sensory signaling in the aging human bowel. Neurogastroenterol Motil. 2016;28(10):1465-1479. doi:10.1111/nmo.12842

184. Lee KJ, Kim YB, Kim JH, Kwon HC, Kim DK, Cho SW. The alteration of enterochromaffin cell, mast cell, and lamina propria $\mathrm{T}$ lymphocyte numbers in irritable bowel syndrome and its relationship with psychological factors. J Gastroenterol Hepatol. 2008;23(11):1689-1694. doi:10.1111/j.1440-1746.2008.05574.x

185. Sander LE, Lorentz A, Sellge G, et al. Selective expression of histamine receptors H1R, H2R, and H4R, but not H3R, in the human intestinal tract. Gut. 2006;55(4):498-504. doi:10.1136/gut.2004.061762

186. Barbara G, Wang B, Stanghellini V, et al. Mast Cell-Dependent Excitation of VisceralNociceptive Sensory Neurons in Irritable Bowel Syndrome. Gastroenterology. 2007;132(1):26-37. doi:10.1053/j.gastro.2006.11.039

187. Lotz M, Vaughan JH, Carson DA. Effect of neuropeptides on production of inflammatory cytokines by human monocytes. Science. 1988;241(4870):1218-1221.

doi:10.1126/science. 2457950

188. O'Malley D, Cryan JF, Dinan TG. Crosstalk between interleukin-6 and corticotropinreleasing factor modulate submucosal plexus activity and colonic secretion. Brain Behav Immun. 2013;30(2013):115-124. doi:10.1016/j.bbi.2013.01.078

189. O’Malley D, Buckley MM, McKernan DP, Quigley EMM, Cryan JF, Dinan TG. Soluble mediators in plasma from irritable bowel syndrome patients excite rat submucosal neurons. Brain Behav Immun. 2015;44:57-67. doi:10.1016/j.bbi.2014.08.005 
190. Gougeon PY, Lourenssen S, Han TY, Nair DG, Ropeleski MJ, Blennerhassett MG. The pro-inflammatory cytokines IL- $1 \beta$ and TNF $\alpha$ are neurotrophic for enteric neurons. $J$ Neurosci. 2013;33(8):3339-3351. doi:10.1523/JNEUROSCI.3564-12.2013

191. Margolis KG, Karamooz N, Stevanovic K, et al. Enteric Neuronal Density Contributes to the Severity of Intestinal Inflammation. Gastroenterology. 2011;141(2):588-598. doi:10.1053/j.gastro.2011.04.047

192. Lückhoff A, Horster M. Hormonal regulation of electrolyte and water transport in the colon. Klin Wochenschr. 1984;62(12):555-563. doi:10.1007/BF01728173

193. Dooley R, Harvey BJ, Thomas W. Non-genomic actions of aldosterone: From receptors and signals to membrane targets. Mol Cell Endocrinol. 2012;350(2):223-234. doi:10.1016/j.mce.2011.07.019

194. Islam S, Abély M, Alam NH, Dossou F, Chowdhury AKA, Desjeux J-F. Water and electrolyte salvage in an animal model of dehydration and malnutrition. J Pediatr Gastroenterol Nutr. 2004;38(1):27-33. doi:10.1097/00005176-200401000-00009

195. Choijookhuu N, Sato Y, Nishino T, Endo D, Hishikawa Y, Koji T. Estrogen-dependent regulation of sodium/hydrogen exchanger-3 (NHE3) expression via estrogen receptor $\beta$ in proximal colon of pregnant mice. Histochem Cell Biol. 2012;137(5):575-587. doi:10.1007/s00418-012-0935-2

196. Ramamoorthy S, Cidlowski JA. Corticosteroids: Mechanisms of Action in Health and Disease. Rheum Dis Clin North Am. 2016;42(1):15-31, vii. doi:10.1016/j.rdc.2015.08.002

197. Hunter RW, Ivy JR, Bailey MA. Glucocorticoids and renal Na+ transport: implications for hypertension and salt sensitivity. J Physiol. 2014;592(8):1731-1744. doi:10.1113/jphysiol.2013.267609

198. Yamazaki O, Ishizawa K, Hirohama D, Fujita T, Shibata S. Electrolyte transport in the renal collecting duct and its regulation by the renin-angiotensin-aldosterone system. Clin Sci (Lond). 2019;133(1):75-82. doi:10.1042/CS20180194

199. Turcu AF, Auchus RJ. Adrenal steroidogenesis and congenital adrenal hyperplasia. Endocrinol Metab Clin North Am. 2015;44(2):275-296. doi:10.1016/j.ecl.2015.02.002

200. Chourpiliadis C, Aeddula NR. Physiology, Glucocorticoids. In: Treasure Island (FL); 2021.

201. Dores RM. Adrenocorticotropic hormone, melanocyte-stimulating hormone, and the melanocortin receptors: revisiting the work of Robert Schwyzer: a thirty-year retrospective. Ann N Y Acad Sci. 2009;1163:93-100. doi:10.1111/j.17496632.2009.04434.x

202. Tsigos C, Chrousos GP. Hypothalamic-pituitary-adrenal axis, neuroendocrine factors and stress. J Psychosom Res. 2002;53(4):865-871. doi:10.1016/s0022-3999(02)00429-4 
203. Bhatia V, Tandon, Rakesh K. Stress and the gastrointestinal tract. J Gastroenterol Hepatol. 2005;20:332-339. doi:10.1111/j.1400-1746.2004.03508.x

204. Mirabito Colafella KM, Bovée DM, Danser AHJ. The renin-angiotensin-aldosterone system and its therapeutic targets. Exp Eye Res. 2019;186:107680.

doi:10.1016/j.exer.2019.05.020

205. Bollag WB. Regulation of aldosterone synthesis and secretion. Compr Physiol. 2014;4(3):1017-1055. doi:10.1002/cphy.c130037

206. Tumamian SG, Binder HJ. Regulation of active sodium and potassium transport in the distal colon of the rat. J Clin Invest. 1989;84(6):1924-1929.

207. Sheppard KE. Nuclear receptors. II. Intestinal corticosteroid receptors. Am J Physiol Gastrointest Liver Physiol. 2002;282(5 45-5):742-746. doi:10.1152/ajpgi.00531.2001

208. Williams DM. Clinical Pharmacology of Corticosteroids. Respir Care. 2018;63(6):655670. doi: $10.4187 /$ respcare. 06314

209. Katz JA. Treatment of inflammatory bowel disease with corticosteroids. Gastroenterol Clin North Am. 2004;33(2):171-189, vii. doi:10.1016/j.gtc.2004.02.002

210. Funder JW. Glucocorticoid receptors. J Steroid Biochem Mol Biol. 1992;43(5):389-394. doi:10.1016/0960-0760(92)90074-s

211. Funder JW. Mineralocorticoid receptors: distribution and activation. Heart Fail Rev. 2005;10(1):15-22. doi:10.1007/s10741-005-2344-2

212. Chapman K, Holmes M, Seckl J. 11ß-hydroxysteroid dehydrogenases: intracellular gatekeepers of tissue glucocorticoid action. Physiol Rev. 2013;93(3):1139-1206. doi:10.1152/physrev.00020.2012

213. White PC, Mune T, Agarwal AK. 11 beta-Hydroxysteroid dehydrogenase and the syndrome of apparent mineralocorticoid excess. Endocr Rev. 1997;18(1):135-156. doi:10.1210/edrv.18.1.0288

214. Cho JH, Musch MW, DePaoli AM, et al. Glucocorticoids regulate $\mathrm{Na}+/ \mathrm{H}+$ exchange expression and activity in region- and tissue-specific manner. Am J Physiol - Cell Physiol. 1994;267(3 36-3). doi:10.1152/ajpcell.1994.267.3.c796

215. Turnamian SG, Binder HJ. Aldosterone and glucocorticoid receptor-specific agonists regulate ion transport in rat proximal colon. Am J Physiol. 1990;258(3 Pt 1):G492-8. doi:10.1152/ajpgi.1990.258.3.G492

216. Cho JH, Musch MW, Bookstein CM, McSwine RL, Rabenau K, Chang EB. Aldosterone stimulates intestinal $\mathrm{Na}+$ absorption in rats by increasing NHE3 expression of the proximal colon. Am J Physiol - Cell Physiol. 1998;274(3 43-3):586-594. doi:10.1152/ajpcell.1998.274.3.c586 
217. Kiela PR, Guner YS, Xu H, Collins JF, Ghishan FK. Age- and tissue-specific induction of NHE3 by glucocorticoids in the rat small intestine. Am J Physiol - Cell Physiol. 2000;278(4 47-4):629-637. doi:10.1152/ajpcell.2000.278.4.c629

218. Kandasamy RA, Orlowski J. Genomic organization glucocorticoid transcriptional activation of the rat $\mathrm{Na}+\mathrm{H}+$ exchanger Nhe3 gene. J Biol Chem. 1996;271(18):1055110559. doi:10.1074/jbc.271.18.10551

219. Malakooti J, Memark VC, Dudeja PK, Ramaswamy K. Molecular cloning and functional analysis of the human $\mathrm{Na}+/ \mathrm{H}+$ exchanger NHE3 promoter. Am J Physiol - Gastrointest Liver Physiol. 2002;282(3 45-3):491-500. doi:10.1152/ajpgi.00273.2001

220. Sandle GI. Segmental variability of glucocorticoid induced electrolyte transport in rat colon. Gut. 1991;32(8):936-940. doi:10.1136/gut.32.8.936

221. Sandle GI, Hayslett JP, Binder HJ. Effect of glucocorticoids on rectal transport in normal subjects and patients with ulcerative colitis. Gut. 1986;27(3):309-316. doi:10.1136/gut.27.3.309

222. Sandle GI, Binder HJ. Corticosteroids and intestinal ion transport. Gastroenterology. 1987;93(1):188-196. doi:10.1016/0016-5085(87)90333-7

223. Bastl CP. Regulation of cation transport by low doses of glucocorticoids in in vivo adrenalectomized rat colon. J Clin Invest. 1987;80(2):348-356. doi:10.1172/JCI113079

224. Bastl CP, Binder HJ, Hayslett JP. Role of glucocorticoids and aldosterone in maintenance of colonic cation transport. Am J Physiol. 1980;238(3):F181-6. doi:10.1152/ajprenal.1980.238.3.F181

225. Bastl CP, Bressler L, Schulman G, Mendez M, Cragoe EJJ. Low-dose glucocorticoids maintain Na-H exchange in distal colon of adrenalectomized rats. Am J Physiol. 1991;261(3 Pt 2):F545-53. doi:10.1152/ajprenal.1991.261.3.F545

226. Rajendran VM, Black J, Ardito TA, et al. Regulation of DRA and AE1 in rat colon by dietary Na depletion. Am J Physiol Gastrointest Liver Physiol. 2000;279(5):G931-42. doi:10.1152/ajpgi.2000.279.5.G931

227. Fukushima K, Sasaki I, Sato S, Sasano H, Krozowski Z, Matsuno S. Induction of mineralocorticoid receptor by sodium butyrate in small intestinal (IEC6) and colonic (T84) epithelial cell lines. Dig Dis Sci. 1999;44(8):1571-1578.

doi:10.1023/a:1026606809171

228. Bergann T, Plöger S, Fromm A, et al. A colonic mineralocorticoid receptor cell model expressing epithelial Na+ channels. Biochem Biophys Res Commun. 2009;382(2):280-285. doi:10.1016/j.bbrc.2009.03.006

229. Zemanová Z, Pácha J. Localization of Na,K-ATPase activity in developing rat distal colon: role of corticosteroids. Mech Ageing Dev. 1998;101(1-2):129-143. 
doi:10.1016/s0047-6374(97)00170-x

230. Escoubet B, Coureau C, Bonvalet JP, Farman N. Noncoordinate regulation of epithelial na channel and Na pump subunit mRNAs in kidney and colon by aldosterone. Am J Physiol Cell Physiol. 1997;272(5 41-5). doi:10.1152/ajpcell.1997.272.5.c1482

231. Musch MW, Lucioni A, Chang EB. Aldosterone regulation of intestinal Na absorption involves SGK-mediated changes in NHE3 and Na+ pump activity. Am J Physiol Gastrointest Liver Physiol. 2008;295(5):909-919. doi:10.1152/ajpgi.90312.2008

232. Bhargava A, Fullerton MJ, Myles K, et al. The serum- and glucocorticoid-induced kinase is a physiological mediator of aldosterone action. Endocrinology. 2001;142(4):15871594. doi:10.1210/endo.142.4.8095

233. Goldschmidt I, Grahammer F, Warth R, et al. Kidney and colon electrolyte transport in CHIF knockout mice. Cell Physiol Biochem Int J Exp Cell Physiol Biochem Pharmacol. 2004;14(1-2):113-120. doi:10.1159/000076932

234. Brennan FE, Fuller PJ. Transcriptional control by corticosteroids of CHIF gene expression in the rat distal colon. Clin Exp Pharmacol Physiol. 1999;26(5-6):489-491. doi:10.1046/j.1440-1681.1999.03065.x

235. Halevy J, Budinger ME, Hayslett JP, Binder HJ. Role of Aldosterone in the Regulation of Sodium and Chloride Transport in the Distal Colon of Sodium-Depleted Rats. Gastroenterology. 1986;91(5):1227-1233. doi:https://doi.org/10.1016/S00165085(86)80021-X

236. Greig ER, Baker EH, Mathialahan T, Boot-Handford RP, Sandle GI. Segmental variability of ENaC subunit expression in rat colon during dietary sodium depletion. Pflugers Arch Eur J Physiol. 2002;444(4):476-483. doi:10.1007/s00424-002-0828-7

237. Garty H, Palmer LG. Epithelial sodium channels: Function, structure and regulation. Physiol Rev. 1997;77(2):359-396. doi:10.1152/physrev.1997.77.2.359

238. Asher C, Wald H, Rossier BC, Garty H. Aldosterone-induced increase in the abundance of $\mathrm{Na}+$ channel subunits. Am J Physiol. 1996;271(2 Pt 1):C605-11. doi:10.1152/ajpcell.1996.271.2.C605

239. Debonneville C, Flores SY, Kamynina E, et al. Phosphorylation of Nedd4-2 by Sgk1 regulates epithelial $\mathrm{Na}+$ channel cell surface expression. EMBO J. 2001;20(24):70527059. doi:10.1093/emboj/20.24.7052

240. Amasheh S, Epple HJ, Mankertz J, et al. Differential Regulation of ENaC by Aldosterone in Rat Early and Late Distal Colon. Ann N Y Acad Sci. 2006;915(1):92-94. doi:https://doiorg.wvu.idm.oclc.org/10.1111/j.1749-6632.2000.tb05227.x

241. Ikuma M, Kashgarian M, Binder HJ, Rajendran VM. Differential regulation of NHE isoforms by sodium depletion in proximal and distal segments of rat colon. Am J Physiol - 
Gastrointest Liver Physiol. 1999;276(2 39-2):539-549. doi:10.1152/ajpgi.1999.276.2.g539

242. Charney AN, Wallach J, Ceccarelli S, Donowitz M, Costenbader CL. Effects of spironolactone and amiloride on corticosteroid-induced changes in colonic function. Am J Physiol. 1981;241(4):G300-5. doi:10.1152/ajpgi.1981.241.4.G300

243. Horster M, Lückhoff A. Aldosterone on sodium transport of rat distal colon in long-term adrenalectomy during acute and chronic substitution. J Physiol. 1983;340:503-511. doi:10.1113/jphysiol.1983.sp014776

244. Patel S, Rauf A, Khan H, Abu-Izneid T. Renin-angiotensin-aldosterone (RAAS): The ubiquitous system for homeostasis and pathologies. Biomed Pharmacother. 2017;94:317325. doi:10.1016/j.biopha.2017.07.091

245. Bandulik S, Tauber P, Lalli E, Barhanin J, Warth R. Two-pore domain potassium channels in the adrenal cortex. Pflugers Arch Eur J Physiol. 2015;467(5):1027-1042. doi:10.1007/s00424-014-1628-6

246. Sørensen M V, Matos JE, Sausbier M, et al. Aldosterone increases KCa1.1 (BK) channelmediated colonic K+ secretion. J Physiol. 2008;586(Pt 17):4251-4264. doi:10.1113/jphysiol.2008.156968

247. Harvey BJ, Alzamora R, Healy V, Renard C, Doolan CM. Rapid responses to steroid hormones: from frog skin to human colon. A homage to Hans Ussing. Biochim Biophys Acta. 2002;1566(1-2):116-128. doi:10.1016/s0005-2736(02)00589-8

248. Doolan CM, O’Sullivan GC, Harvey BJ. Rapid effects of corticosteroids on cytosolic protein kinase $\mathrm{C}$ and intracellular calcium concentration in human distal colon. Mol Cell Endocrinol. 1998;138(1-2):71-79. doi:10.1016/s0303-7207(98)00020-3

249. Doolan CM, Harvey BJ. Modulation of cytosolic protein kinase $\mathrm{C}$ and calcium ion activity by steroid hormones in rat distal colon. J Biol Chem. 1996;271(15):8763-8767. doi:10.1074/jbc.271.15.8763

250. Maguire D, MacNamara B, Cuffe JE, et al. Rapid responses to aldosterone in human distal colon. Steroids. 1999;64(1-2):51-63. doi:10.1016/s0039-128x(98)00096-8

251. Bowley KA, Linley JE, Robins GG, Kopanati S, Hunter M, Sandle GI. Role of protein kinase $\mathrm{C}$ in aldosterone-induced non-genomic inhibition of basolateral potassium channels in human colonic crypts. J Steroid Biochem Mol Biol. 2007;104(1-2):45-52. doi:10.1016/j.jsbmb.2006.10.003

252. Corleto VD. Somatostatin and the gastrointestinal tract. Curr Opin Endocrinol Diabetes Obes. 2010;17(1):63-68. doi:10.1097/MED.0b013e32833463ed

253. Warhurst G, Higgs NB, Fakhoury H, Warhurst AC, Garde J, Coy DH. Somatostatin receptor subtype 2 mediates somatostatin inhibition of ion secretion in rat distal colon. Gastroenterology. 1996;111(2):325-333. doi:10.1053/gast.1996.v111.pm8690197 
254. Ferrar JA, Cuthbert AW, Cox HM. The antisecretory effects of somatostatin and analogues in rat descending colon mucosa. Eur J Pharmacol. 1990;184(2-3):295-303. doi:10.1016/0014-2999(90)90621-c

255. Dharmsathaphorn K, Racusen L, Dobbins JW. Effect of somatostatin on ion transport in the rat colon. J Clin Invest. 1980;66(4):813-820. doi:10.1172/JCI109919

256. Diener M, Gartmann V. Effect of somatostatin on cell volume, Cl- currents, and transepithelial Cl- transport in rat distal colon. Am J Physiol. 1994;266(6 Pt 1):G1043-52. doi:10.1152/ajpgi.1994.266.6.G1043

257. Perry MD, Sandle GI. Regulation of colonic apical potassium (BK) channels by cAMP and somatostatin. Am J Physiol Gastrointest Liver Physiol. 2009;297(1):G159-G167. doi:10.1152/ajpgi.00132.2009

258. Gomes-Porras M, Cárdenas-Salas J, Álvarez-Escolá C. Somatostatin analogs in clinical practice: A review. Int J Mol Sci. 2020;21(5):1-27. doi:10.3390/ijms21051682

259. Wada-Hiraike $\mathrm{O}$, Imamov $\mathrm{O}$, Hiraike $\mathrm{H}$, et al. Role of estrogen receptor $\beta$ in colonic epithelium. Proc Natl Acad Sci U S A. 2006;103(8):2959-2964. doi:10.1073/pnas.0511271103

260. Tutton PJ, Barkla DH. Differential effects of oestrogenic hormones on cell proliferation in the colonic crypt epithelium and in colonic carcinomata of rats. Anticancer Res. 1982;2(4):199-201.

261. Braniste V, Leveque M, Buisson-Brenac C, Bueno L, Fioramonti J, Houdeau E. Oestradiol decreases colonic permeability through oestrogen receptor beta-mediated upregulation of occludin and junctional adhesion molecule-A in epithelial cells. $J$ Physiol. 2009;587(Pt 13):3317-3328. doi:10.1113/jphysiol.2009.169300

262. Leiszter K, Galamb O, Kalmár A, et al. [Potential role of estrogens in colorectal tumour development]. Orv Hetil. 2020;161(14):532-543. doi:10.1556/650.2020.31674

263. Harvey BJ, Doolan CM, Condliffe SB, Renard C, Alzamora R, Urbach V. Non-genomic convergent and divergent signalling of rapid responses to aldosterone and estradiol in mammalian colon. Steroids. 2002;67(6):483-491. doi:10.1016/s0039-128x(01)00169-6

264. Doolan CM, Condliffe SB, Harvey BJ. Rapid non-genomic activation of cytosolic cyclic AMP-dependent protein kinase activity and $[\mathrm{Ca}(2+)]$ (i) by 17 beta-oestradiol in female rat distal colon. Br J Pharmacol. 2000;129(7):1375-1386. doi:10.1038/sj.bjp.0703193

265. Condliffe SB, Doolan CM, Harvey BJ. 17beta-oestradiol acutely regulates Cl- secretion in rat distal colonic epithelium. J Physiol. 2001;530(Pt 1):47-54. doi:10.1111/j.1469$7793.2001 .0047 \mathrm{~m} . \mathrm{x}$

266. O'Mahony F, Thomas W, Harvey BJ. Novel female sex-dependent actions of oestrogen in the intestine. J Physiol. 2009;587(21):5039-5044. doi:10.1113/jphysiol.2009.177972 
267. O'Mahony F, Alzamora R, Chung H-L, Thomas W, Harvey BJ. Genomic Priming of the Antisecretory Response to Estrogen in Rat Distal Colon throughout the Estrous Cycle. $J$ Clin Endocrinol Metab. 2009;94(11):4627-4628. doi:10.1210/jcem.94.11.9991

268. Rapetti-Mauss R, O’Mahony F, Sepulveda F V, Urbach V, Harvey BJ. Oestrogen promotes KCNQ1 potassium channel endocytosis and postendocytic trafficking in colonic epithelium. J Physiol. 2013;591(11):2813-2831. doi:10.1113/jphysiol.2013.251678

269. Alzamora R, O’Mahony F, Harvey BJ. Estrogen inhibits chloride secretion caused by cholera and Escherichia coli enterotoxins in female rat distal colon. Steroids. 2011;76(9):867-876. doi:10.1016/j.steroids.2011.04.016

270. Mayol JM, Arbeo-Escolar A, Alarma-Estrany P, Adame-Navarrete Y, Fernández-Represa JA. Progesterone inhibits chloride transport in human intestinal epithelial cells. World $J$ Surg. 2002;26(6):652-656. doi:10.1007/s00268-001-0284-0 
A

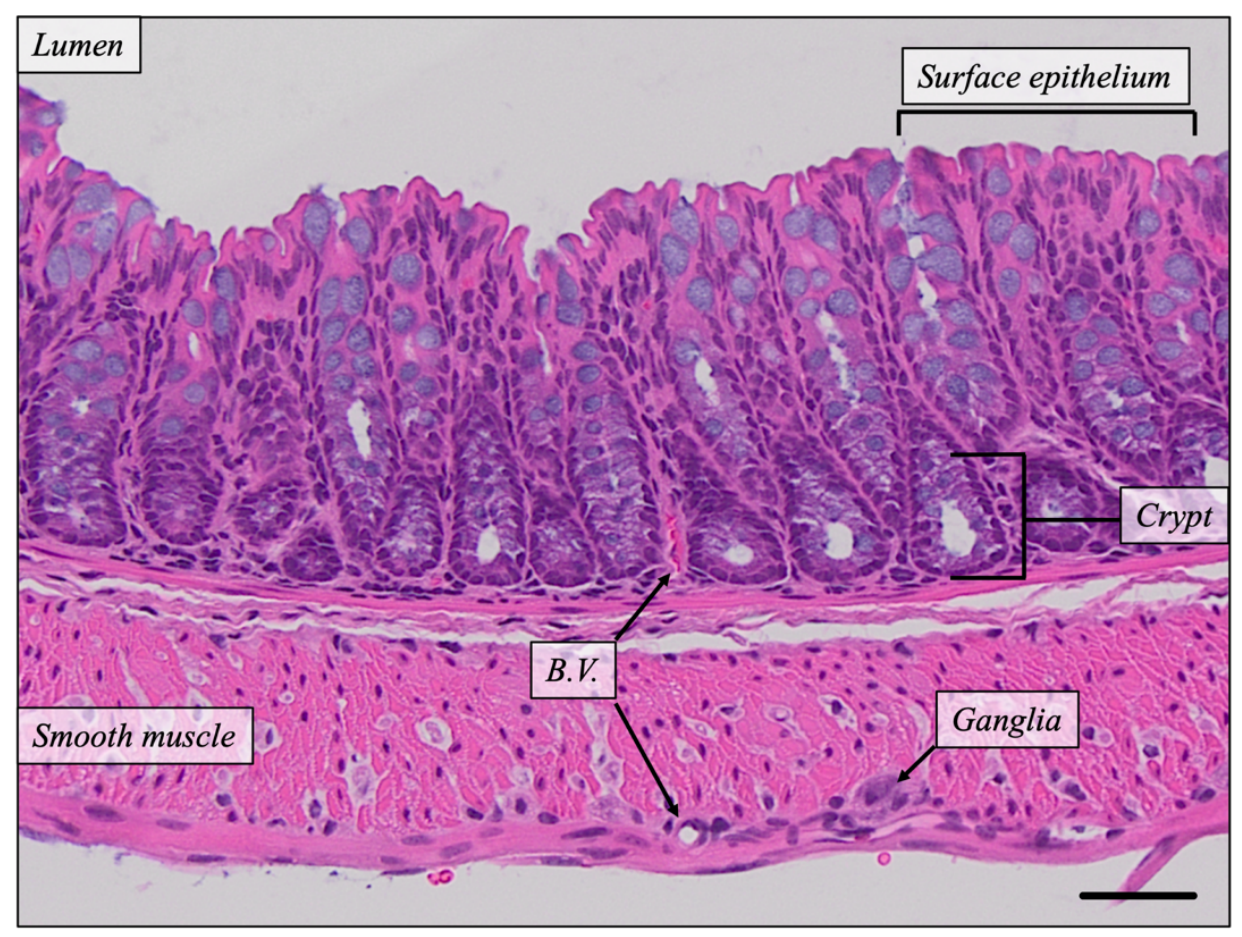

B

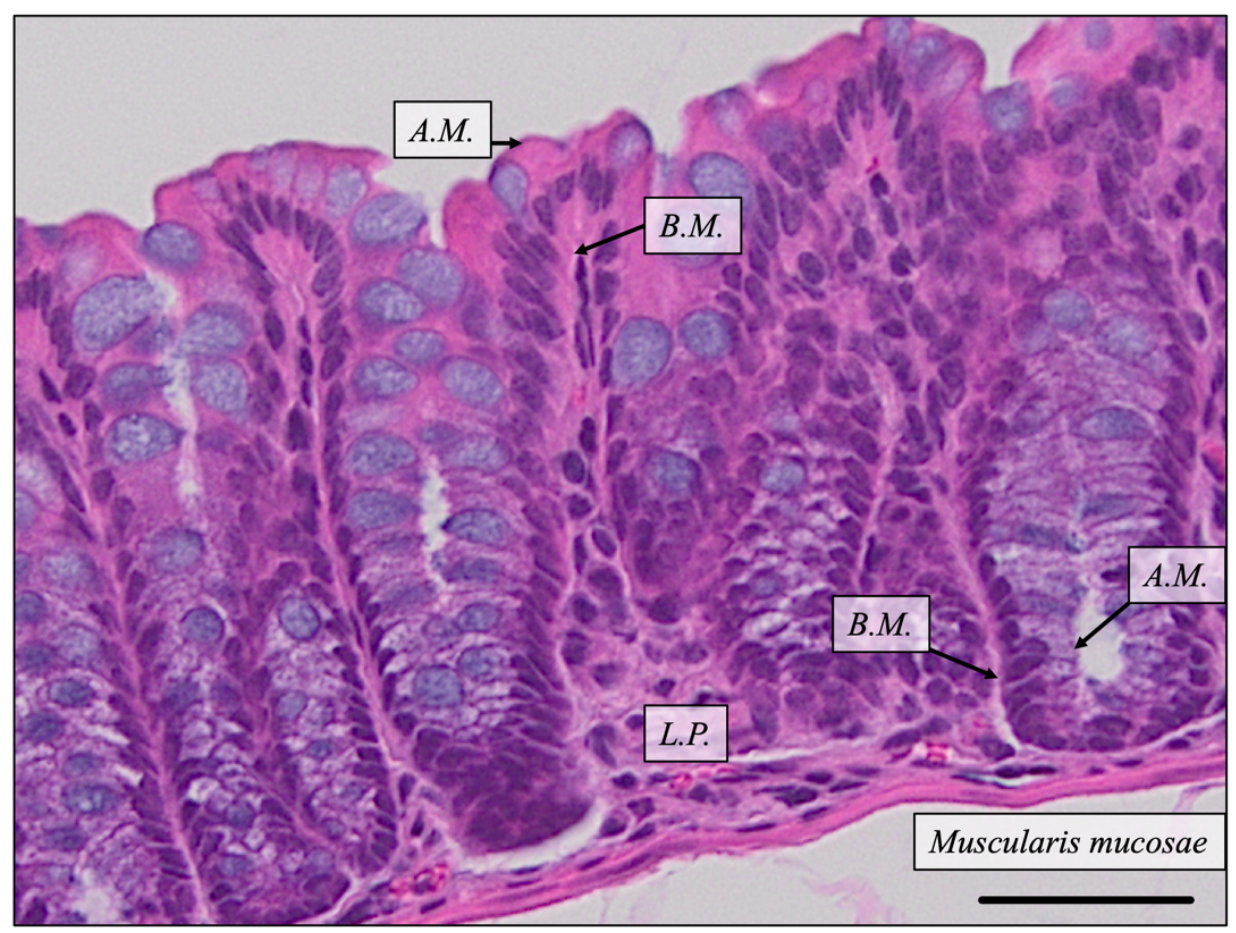


Figure 1 - Histological structure of the colonic epithelium. Mouse colonic epithelium stained with hematoxylin and eosin and sectioned at $5 \mu \mathrm{m}$. Images captured at 20x magnification; scale bars $=50 \mu \mathrm{m} . A$ : Full-thickness colon tissue depicted, with surface and crypt epithelial regions shown, as well as underlying submucosa and smooth muscle containing blood vessels and ganglia. B.V.= blood vessel; A.M. = apical membrane; B.M. = basolateral membrane; L.P. = lamina propria.

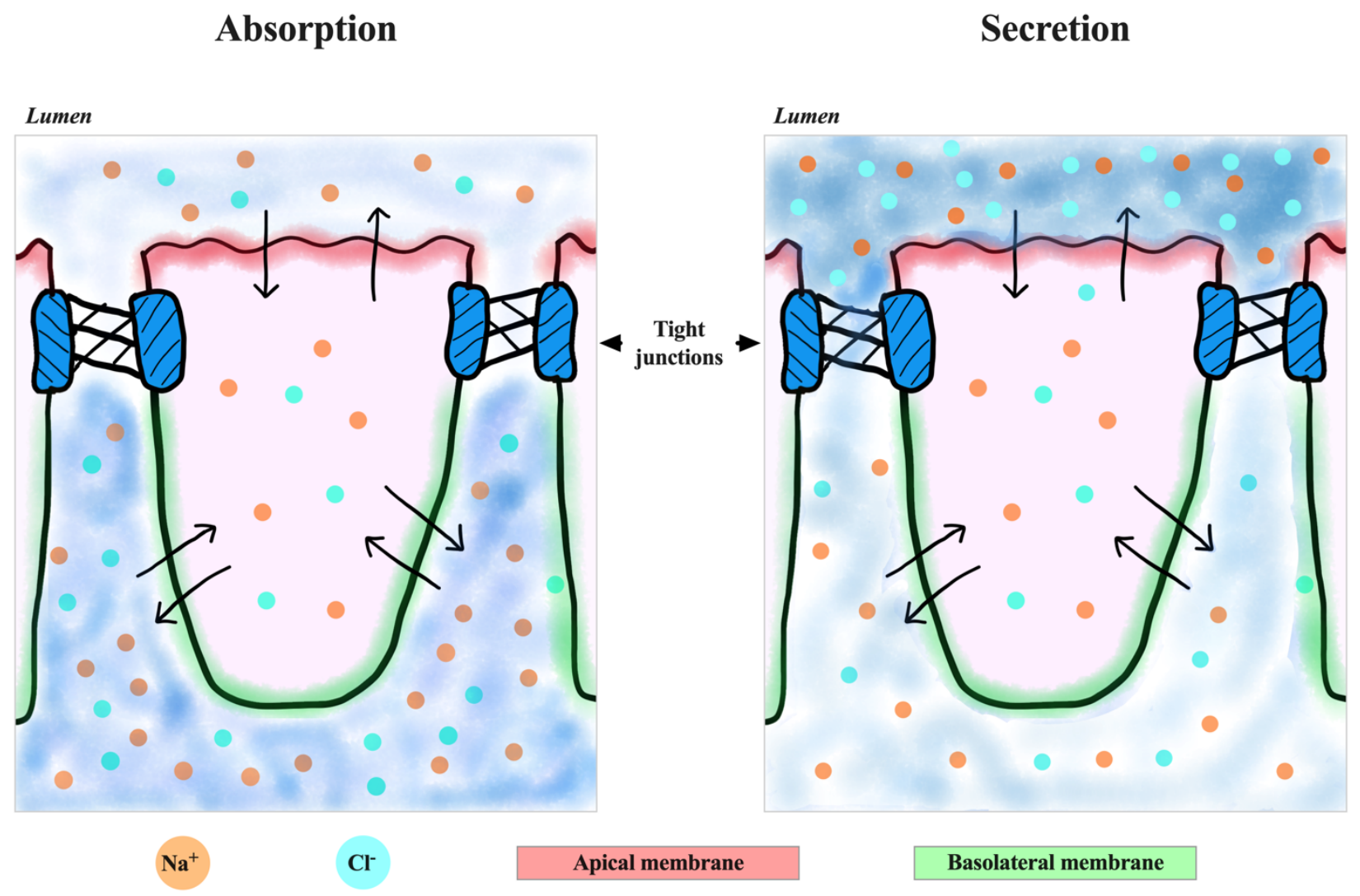


Figure 2 - Basic structure and function of colonic epithelial cells. Individual epithelial cells of the colon (colonocytes) are connected to adjacent cells via tight junction (shown in blue) to form a tight barrier which lines the gut. Net absorption of electrolyte ions, such as $\mathrm{Na}^{+}$and $\mathrm{Cl}^{-}$, through the trans-cellular compartment and into the interstitial space allows for water absorption to occur through osmosis (left panel). Conversely, secretion of electrolytes into the luminal space drives water secretion (right panel). Electrolyte movement in either direction requires transport (either active or passive) across the apical and basolateral cell membranes, shown here in red and green, respectively. Water transport also occurs largely through the trans-cellular compartment. The colon is capable of both absorption and secretion, depending upon the needs of the organism. 
A Electroneutral $\mathrm{NaCl}$ absorption (proximal/mid-distal colon)

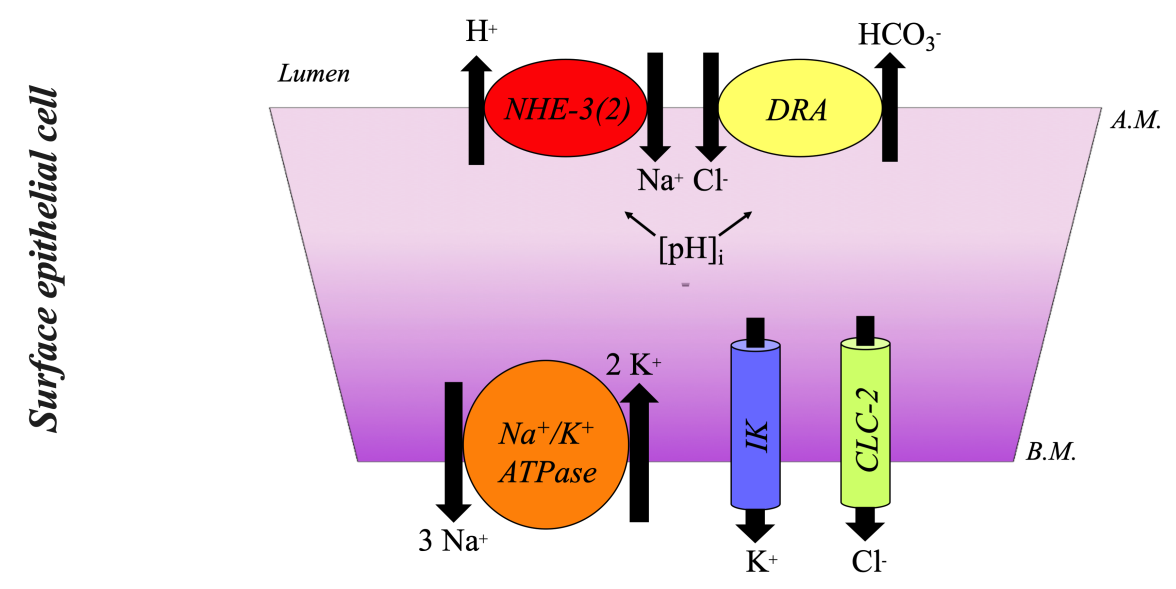

B

Electrogenic $\mathrm{Na}^{+}$absorption and $\mathrm{K}^{+}$secretion (distal colon)

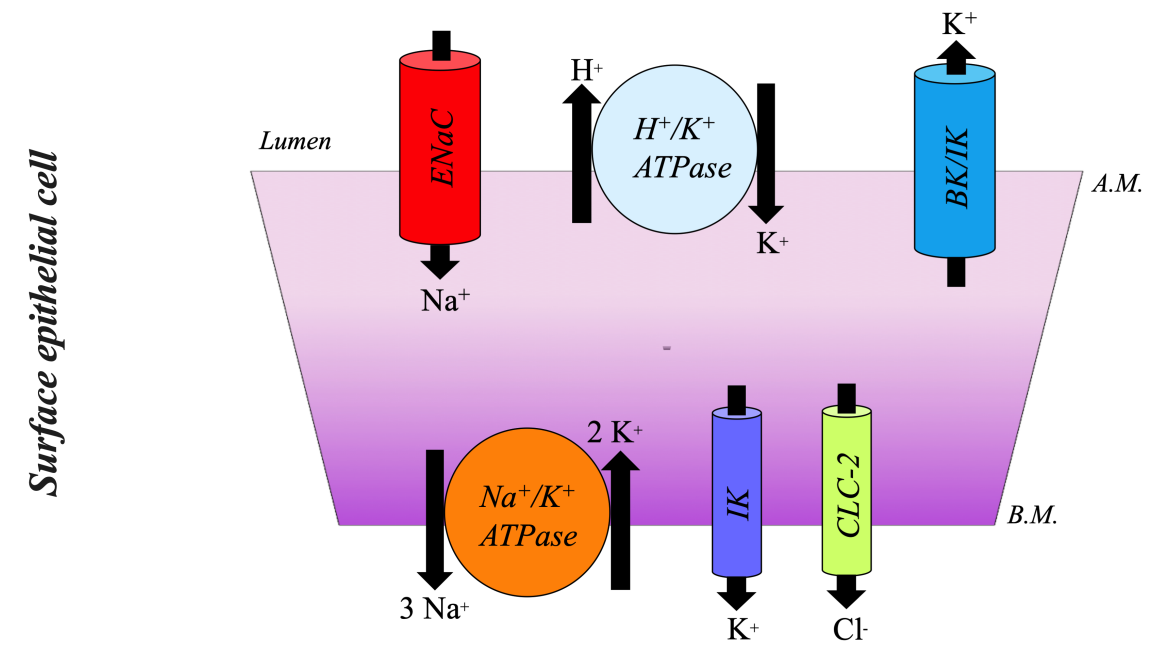

C

Electrogenic $\mathrm{Cl}^{-}$and $\mathrm{K}^{+}$secretion (proximal and distal colon)

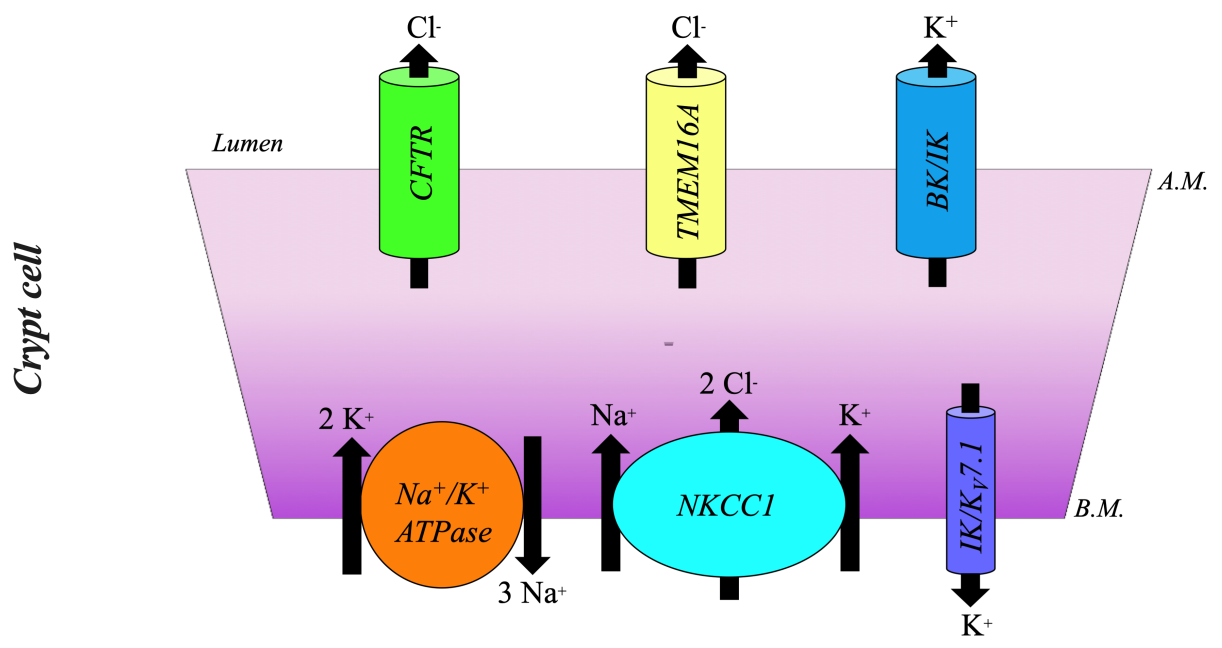




\section{Figure 3-Summary of the major electrolyte transport systems within the colonic epithelium.}

$A$ : Electroneutral $\mathrm{NaCl}$ absorption occurs in surface epithelial cells of the proximal and mid-distal colon. $\mathrm{Na}^{+} / \mathrm{H}^{+}$exchanger isoform 3 (NHE-3) and the "down-regulated in adenoma" $\mathrm{Cl}^{-} / \mathrm{HCO}_{3}{ }^{-}$ exchanger (DRA) mediate electroneutral $\mathrm{Na}^{+}$and $\mathrm{Cl}^{-}$absorption, respectively, which are coupled via intracellular $\mathrm{pH}$. Basolateral $\mathrm{Na}^{+} / \mathrm{K}^{+}$ATPase activity drives this process by continuously extruding $\mathrm{Na}^{+}$across the basolateral membrane. Intermediate conductance $\mathrm{K}^{+}(\mathrm{IK})$ and $\mathrm{Cl}^{-}$channel 2 (CLC-2) channels primarily facilitate electroneutral $\mathrm{K}^{+}$and $\mathrm{Cl}^{-}$uptake across the basolateral membrane. $B$ : Electrogenic $\mathrm{Na}^{+}$absorption occurs via the epithelial $\mathrm{Na}^{+}$channel $(\mathrm{ENaC})$ in surface epithelial cells of the distal colon. Apical membrane $\mathrm{H}^{+} / \mathrm{K}^{+}$ATPase and large conductance $\mathrm{K}^{+}$ (BK) channels mediate active $\mathrm{K}^{+}$absorption and secretion, respectively. Basolateral transporter/channel functions are similar to panel $A$. C: Electrogenic $\mathrm{Cl}^{-}$and $\mathrm{K}^{+}$secretion occurs within the crypt region of the colonic epithelium in both proximal and distal regions of the tissue. Apical membrane BK channels and cystic fibrosis transmembrane conductance regulator (CFTR) and transmembrane protein 16A (TMEM16A) likely mediate efflux of $\mathrm{K}^{+}$and $\mathrm{Cl}^{-}$, respectively. Basolateral $\mathrm{Na}^{+} / \mathrm{K}^{+}$ATPase activity drives $\mathrm{K}^{+}$and $\mathrm{Cl}^{-}$uptake via the $\mathrm{NKCC1}$ co-transporter, which ultimately supports active secretion. Basolateral IK or voltage gated $\mathrm{K}_{\mathrm{V}} 7.1$ channels participate in $\mathrm{K}^{+}$recycling in conjunction with $\mathrm{NKCC} 1$ in order to maintain the electrical driving force necessary for sustained $\mathrm{Cl}^{-}$efflux. 


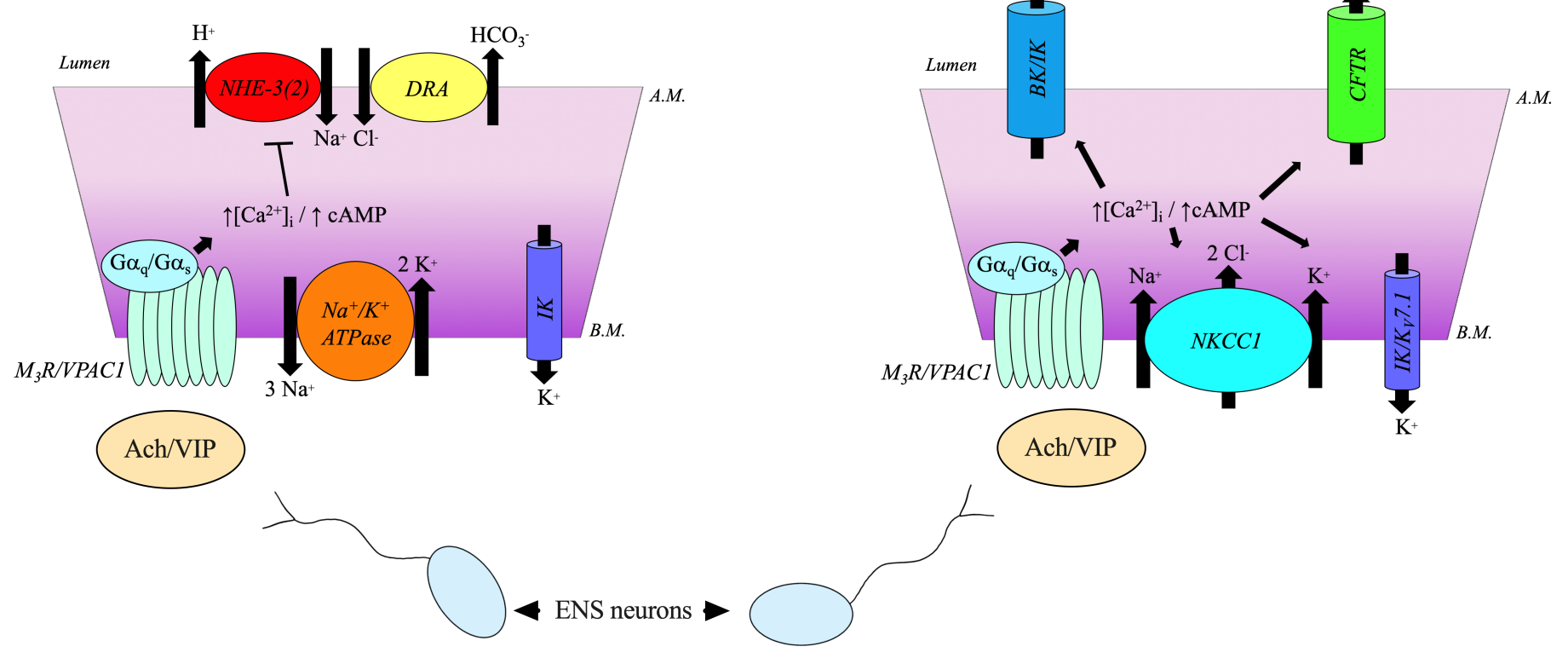

Figure 4 - Enteric nervous system (ENS) input generally inhibits $\mathrm{Na}^{+}$absorption and stimulates $\mathrm{Cl}^{-}$secretion in the colonic epithelium. Release of ENS neurotransmitters (e.g., acetylcholine; Ach, or vasoactive intestinal peptide; VIP) stimulates basolateral receptors (e.g., $\mathrm{M}_{3} \mathrm{R}$ and VPAC1, respectively) on colonic epithelial cells. These receptors are typically linked to Gaq (M3R) or Gas (VPAC1), which induce $\mathrm{Ca}^{2+}$ - and/or cAMP-dependent signaling events, respectively, leading to inhibition of $\mathrm{Na}^{+}$absorption and stimulation of $\mathrm{Cl}^{-}$secretion. This outcome is largely the result of trafficking of channels and transporters to (e.g., NKCC1) and from (e.g., NHE-3) the plasma membrane to regulate their activities. In addition, protein kinase A or C (PKA/PKC)-induced phosphorylation of channels, transporters or regulatory complex proteins is often a critical regulatory component. The end result of these events is typically decreased $\mathrm{NaCl}$ absorption, which leads to decreased water absorption and increased stool volume (i.e. diarrhea). 


\section{CHAPTER 2}

Andrew J. Nickerson ${ }^{1,2,}$, Trey S. Rottgen ${ }^{1,2, \omega^{\dagger},}$, Vazhaikkurichi M. Rajendran ${ }^{2,3,{ }^{*}}$

${ }^{1}$ Departments of Physiology, Pharmacology and Neuroscience, West Virginia University School of Medicine, Morgantown, West Virginia.

${ }^{2}$ Department of Biochemistry, West Virginia University School of Medicine, Morgantown, West Virginia.

${ }^{3}$ Department of Medicine, West Virginia University School of Medicine, Morgantown, West Virginia.

"Both authors contributed equally to this study

${ }^{\dagger}$ Current address: Department of Internal Medicine, University of Michigan, Ann Arbor, Michigan

Running title: $\mathrm{KCNQ}\left(\mathrm{K}_{\mathrm{V}} 7\right)$ activation in ENS neurons inhibits epithelial $\mathrm{Cl}^{-}$secretion

\section{*Address all Correspondence}

Vazhaikkurichi M. Rajendran, PhD.

Departments of Biochemistry and Medicine

West Virginia University School of Medicine

1 Medical Center Drive

Morgantown, WV 26506

Phone: (304) 293-0510

Email:vrajendran@hsc.wvu.edu 


\section{ABSTRACT}

$\mathrm{K}_{\mathrm{V}} 7(\mathrm{KCNQ}) \mathrm{K}^{+}$channels are expressed in many neuronal populations, and play an important role in regulating membrane potential by generating a hyper-polarizing $\mathrm{K}^{+}$current and decreasing cell excitability. However, the role of $\mathrm{K}_{\mathrm{V}} 7$ channels in the neural regulation of intestinal epithelial $\mathrm{Cl}^{-}$secretion is not known. $\mathrm{Cl}^{-}$secretion in mouse distal colon was measured as a function of short circuit current $\left(\mathrm{I}_{\mathrm{SC}}\right)$, while pharmacological approaches were used to test the hypothesis that activation of $\mathrm{K}_{\mathrm{V}} 7$ channels in enteric neurons would inhibit epithelial $\mathrm{Cl}^{-}$secretion. Flupirtine, a non-selective $\mathrm{K}_{\mathrm{V}} 7$ activator, inhibited basal $\mathrm{Cl}^{-}$secretion in mouse distal colon and abolished or attenuated the effects of drugs that target various components of enteric neurotransmission, including tetrodotoxin $\left(\mathrm{Na}_{\mathrm{V}}\right.$ channel blocker), Veratridine $\left(\mathrm{Na}_{\mathrm{V}}\right.$ channel activator), Nicotine (nicotinic acetylcholine receptor agonist) and Hexamethonium (nicotinic antagonist). In contrast, flupirtine did not block the response to epithelium-targeted agents VIP (endogenous VPAC receptor ligand) or carbachol (non-selective cholinergic agonist). Flupirtine inhibited $\mathrm{Cl}^{-}$secretion in both full-thickness and seromuscular-stripped distal colon (containing the submucosal, but not myenteric plexus), but generated no response in epithelial T84 cell monolayers. $\mathrm{K}_{\mathrm{V}} 7.2$ and $\mathrm{K}_{\mathrm{V}} 7.3$ channel proteins were detected by immunofluorescence in whole-mount preparations of the submucosa from mouse distal colon. ICA $110381\left(\mathrm{~K}_{\mathrm{V}} 7.2 / 7.3\right.$ specific activator $)$ inhibited $\mathrm{Cl}^{-}$ secretion comparably to flupirtine. We conclude that $\mathrm{K}_{\mathrm{V}} 7$ channel activators inhibit neurallydriven $\mathrm{Cl}^{-}$secretion in the colonic epithelium and may therefore have therapeutic benefit in treating pathologies associated with hyper-excitable enteric nervous system, such as irritable bowel syndrome with diarrhea (IBS-D).

KEY WORDS: Colonic epithelium. $\mathrm{K}_{\mathrm{V}} 7 \mathrm{~K}^{+}$channels . Enteric nervous system (ENS). Irritable bowel syndrome with diarrhea (IBS-D) . Flupirtine. Ussing Chamber . 


\section{INTRODUCTION}

Irritable bowel syndrome with diarrhea (IBS-D) is one of the most common gastrointestinal (GI) disorders (43). Symptoms such as frequent and watery bowel movements are often related to hyperactivity of the enteric nervous system (ENS) $(11,20,53)$. Pharmacological agents that inhibit hyperactivity of secretomotor neurons of the ENS have been shown to increase colonic transit time and reduce stool water content in IBS-D $(24,30,36)$. Although ENS-targeted drug therapies, such as eluxadoline (opioid receptor agonist) or alosetron (5-HT 3 receptor antagonist) alleviate IBS-D symptoms, they are also commonly associated with adverse side effects such as drowsiness, blurred vision and constipation (12). Development of novel therapeutic strategies therefore remains an important and active area of research.

The KCNQ $\left(\mathrm{K}_{\mathrm{V}} 7\right)$ gene family is a group of small conductance, voltage-gated $\mathrm{K}^{+}$channels, which open at characteristically negative membrane potentials $(4,10,46,54)$. It has been shown that some members of $K_{V} 7$ family $\left(K_{V} 7.2, K_{V} 7.3\right.$ and $\left.K_{V} 7.5\right)$ can assemble to form heterotetramers that carry the "M" current, a stabilizing $\mathrm{K}^{+}$current that is inhibited by muscarinic receptor stimulation in neurons $(51,59)$. In addition, targeted KCNQ gene disruptions have been shown to cause seizures and hyperalgia in experimental models, while $\mathrm{K}_{\mathrm{V}} 7$ channel activators that inhibit neural excitability have been used in the treatment of epilepsy $(8,22,50,60) . K_{V} 7$ channels have thus been implicated as important regulators of resting membrane potential in neurons.

$\mathrm{K}_{\mathrm{V}} 7$ channels have recently been localized in neurons of the ENS along the human GI tract, as well as afferent fibers innervating the distal colon in both human and mouse (49). It was demonstrated that neuronal $\mathrm{K}_{\mathrm{V}} 7.3$ activation inhibited both mechanical- and chemical-stimulated sensory response, providing evidence that $\mathrm{K}_{\mathrm{V}} 7$ channels could be a target in the treatment of GI pain. However, the effects of $\mathrm{K}_{\mathrm{V}} 7$ channel activation with respect to epithelial electrolyte transport 
are not known. Because neural stimulation of epithelial $\mathrm{Cl}^{-}$secretion through apical $\mathrm{Cl}^{-}$channels (e.g. CFTR) can provide a driving force for water secretion in the GI tract (i.e. diarrhea) $(17,34)$, there is potential therapeutic value in understanding the role of $\mathrm{K}_{\mathrm{V}} 7$ channels in this process.

We hypothesized that $\mathrm{K}_{\mathrm{V}} 7$ channel activation would dampen stimulatory ENS input to the intestinal epithelium, thus inhibiting epithelial $\mathrm{Cl}^{-}$secretion. To test this hypothesis, we examined the effect of the $\mathrm{K}_{\mathrm{V}} 7$ activator, flupirtine, on $\mathrm{Cl}^{-}$secretion in mouse distal colon. Following the initial observation that flupirtine inhibited basal short-circuit current ( $\mathrm{I}_{\mathrm{SC}}$; i.e. $\mathrm{Cl}^{-}$secretion), pharmacological approaches were used to examine whether flupirtine acted on the ENS or the epithelium directly, and immunofluorescence microscopy was used to confirm the expression of $\mathrm{K}_{\mathrm{V}} 7$ subunits in enteric neurons. The results presented in this study provide evidence that inhibition of epithelial $\mathrm{Cl}^{-}$secretion by flupirtine is likely due to activation of ENS-localized $\mathrm{K}_{\mathrm{V}} 7$ channels.

\section{MATERIALS AND METHODS}

\section{Animals}

Male BALB/c mice (8 weeks old, Charles River, NY) were housed in groups of 2-4, exposed to 12-hour light/dark cycles and provided normal chow and water ad libitum. Female mice were excluded from this study for the purpose of controlling for unwanted variability in $\mathrm{Cl}^{-}$ secretion resulting from female sex hormone activity $(2,14)$. Animals were anesthetized using $5 \%$ isoflurane (Patterson Veterinary, SC) balanced with oxygen, and the whole colon was removed by gently cutting away mesenteric attachments. Animals were then euthanized via cervical dislocation. Tissues harvested from each mouse were randomly assigned to treatment groups. All experimental protocols utilized in this study were approved by the West Virginia University Institutional Animal Care and Use Committee. 


\section{Ussing chamber experiments}

Mouse colonic tissue:

Whole colon was removed from anesthetized mice and flushed gently with ice-cold phosphate-buffered saline (PBS) to remove fecal contents. The colon was then incubated for 5-10 minutes in ice-cold PBS containing $5 \mu \mathrm{M}$ indomethacin to inhibit prostaglandin production during tissue manipulation, before being pinned near the caecum in a sylgard plate. A longitudinal incision was made along the mesenteric border and the tissue was pinned mucosal side down. After removing the attached mesentery, segments of mid-distal colon $(2-5 \mathrm{~cm}$ proximal to the rectum) were then excised and mounted on plastic sliders with an oval aperture of $0.04 \mathrm{~cm}^{2}$ for use in an EasyMount Ussing-style chamber (Physiological Instruments, San Diego, CA). Up to 4 tissues were taken from each colon. For experiments using seromuscular-stripped tissue, a lateral incision was made into the serosal side of the tissue and the musculature was gently peeled back using ultra-fine forceps under a dissecting microscope. Removal of smooth musculature and the myenteric plexus contained within was performed to examine the effects of flupirtine on submucosal neurons, which were left intact in these preparations. Selected segments of unstripped (full-thickness) and seromuscular-stripped colon were set aside for histological assessment, as described below.

Mounted tissues were bathed bilaterally in $5 \mathrm{~mL}$ of modified Kreb's solution containing, in mM: $115 \mathrm{NaCl}, 25 \mathrm{NaHCO}_{3}, 1.6 \mathrm{~K}_{2} \mathrm{HPO}_{4}, 0.4 \mathrm{KH}_{2} \mathrm{PO}_{4}, 2.8 \mathrm{KCl}, 1.2 \mathrm{CaCl}_{2}, 1.2 \mathrm{MgCl}_{2}$ and 10 glucose ( $\mathrm{pH}$ 7.4). Tissues were monitored and allowed to equilibrate under open-circuit conditions for a period of 15-20 minutes until trans-epithelial potential $\left(\mathrm{V}_{\mathrm{TE}}\right)$ had stabilized, while being maintained at $37^{\circ} \mathrm{C}$ and continuously gassed with $5 \% \mathrm{CO}_{2}, 95 \% \mathrm{O}_{2}$. Short-circuit current $\left(\mathrm{I}_{\mathrm{SC}}\right)$ recordings were performed as described previously (52). In brief, all mounted tissues were voltage- 
clamped at $0 \mathrm{mV}$ using a multichannel voltage clamp (VCC MC8; Physiologic Instruments, San Diego, CA) and $\mathrm{I}_{\mathrm{SC}}$ was measured continuously by computer-operated software (Acquire \& Analyze; Physiologic Instruments). A brief (200 ms) bipolar $5 \mathrm{mV}$ pulse was applied every 5 seconds to monitor trans-epithelial resistance. Under these conditions, positive $\mathrm{I}_{\mathrm{SC}}$ values reflect anion $\left(\mathrm{Cl}^{-}\right)$secretion. ISC recordings were allowed to reach a steady-state for at least 5 minutes prior to the addition of any drugs. Up to four adjacent tissues were obtained from each animal. When four tissues were taken from the same animal, the average intra-animal basal Isc range was 56.6 and the average standard deviation was $\pm 25.4 \mu \mathrm{A} / \mathrm{cm}^{2}(\mathrm{n}=10)$.

It should be noted that while electrogenic $\mathrm{Na}^{+}$absorption via epithelial $\mathrm{Na}^{+}$channel $(\mathrm{ENaC})$ can also generate a positive $\mathrm{I}_{\mathrm{SC}}$ in the colon of some species under certain conditions, normal mouse colon exhibits very minimal $\mathrm{ENaC}$ activity and this is restricted to only the most distal segment of the tissue (32). For all experiments in this study, tissues were taken from between 2 and $5 \mathrm{~cm}$ proximal to the rectum (mid-distal colon). Thus, we make the assumption that positive ISC values under our experimental conditions reflect predominantly $\mathrm{Cl}^{-}$secretion. This approach was chosen to eliminate any potential confounding effects of using an $\mathrm{ENaC}$ inhibitor, such as amiloride.

T84 cells

T84 cells (a human colonic adenocarcinoma cell line, ATCC, Manassas, VA) were grown and maintained in DMEM/F12 media (ThermoFisher, Waltham, MA) supplemented with $10 \%$ fetal bovine serum (FBS) (Fisher Scientific, Hampton, NH) and 1\% penicillin-streptomycin (Mediatech, Manassas, VA) at $37^{\circ} \mathrm{C}$ in $5 \% \mathrm{CO}_{2}$ incubator. After passaging, cells were seeded at near confluence onto $1.12 \mathrm{~cm}^{2}$ Snapwell inserts that contain permeable support (Corning, Corning, NY). Upon reaching confluence, the cells were maintained in monolayers for 18 days to ensure 
complete development of tight junctions and trans-epithelial potential $\left(\mathrm{V}_{\mathrm{TE}}\right)$. Fresh culture media was replaced every other day until utilization in Ussing chamber studies. On the day of experiments, the inserts containing T84 monolayers were removed and gently washed with warm PBS. The monolayers were then mounted in the Ussing chamber and bathed on both sides with Ringers' solution. ISC measurements were performed as described above. Monolayers were allowed to equilibrate and come to a steady-state ( $\sim 15$ minutes) prior to the application of any drugs.

Histology

Full-thickness and seromuscular-stripped segments of mid-distal colon were fixed in $4 \%$ paraformaldehyde in PBS for 1 hour at room temperature and embedded in paraffin. The colons were then sectioned $(5 \mu \mathrm{m})$, mounted on glass slides and stained with hematoxylin and eosin for visualization of tissue structures. Images were captured with an Olympus VS210 slide scanner (Tokyo, Japan) using a 20x objective.

\section{Immunofluorescence studies}

To obtain a whole-mount preparation of the submucosal plexus, the distal colon was first opened along the mesenteric border and pinned mucosal side down. Similar to the tissue preparation for experiments using seromuscular-stripped colon, a lateral incision was made into the serosal side and the musculature was peeled away using forceps. The tissue was then placed mucosal side facing upward and the epithelium was removed by scraping with a glass slide. The remaining tissue was then fixed for 48 hours at $4{ }^{\circ} \mathrm{C}$ in $4 \%$ paraformaldehyde in PBS. After permeabilizing with 1\% Triton-X 100 (Fisher Scientific, Hampton, NH) plus 5\% goat serum (Fisher Scientific, Hampton, NH) in PBS for 30 minutes at room temperature, tissues were incubated at $4{ }^{\circ} \mathrm{C}$ overnight in blocking solution (5\% goat serum, $0.01 \%$ Triton- $\mathrm{X}$ in PBS) 
containing the following primary antibodies: mouse anti-microtubule-associated protein 2 (MAP2; Millipore \#MAB3418; 1:200 dilution) plus either rabbit anti-KV7.2 (Alomone \#APC-050; 1:100 dilution) or rabbit anti-KV7.3 (Alomone \#APC-051; 1:100 dilution). All of these commerciallyavailable antibodies have been used in immunofluorescence microscopy experiments to detect their respective proteins in mouse tissues $(39,56,61)$. The following day, tissues were washed 5 times for 5 minutes each with PBS $+0.01 \%$ Triton-X 100 (PBST) before incubating in blocking solution containing AlexaFluor 568 goat anti-mouse (Thermo \#A11031; 1:2,000 dilution) and AlexaFluor 488 goat anti-rabbit (Thermo \#A11008; 1:2,000 dilution) for 30 minutes at room temperature to allow detection of MAP2 (anti-mouse) and $\mathrm{K}_{\mathrm{V}} 7.2$ or $\mathrm{K}_{\mathrm{V}} 7.3$ (anti-rabbit) within the same tissue preparation. After washing again 5 times in PBST for 5 minutes each, the tissues were placed on charged glass slides and mounted in Slow-Fade Diamond anti-fade mountant with DAPI (Thermofisher Scientific, Waltham, MA). Images were captured with a Zeiss 710 confocal microscope (Carl Zeiss, Oberkcehn, Germany) using a 20x objective.

\section{Data analysis}

\section{Flupirtine concentration-response analysis}

Flupirtine was prepared as 1, 10 and $100 \mathrm{mM}$ stock solutions and diluted to reach a final working concentration range of $1 \mathrm{nM}$ to $250 \underline{\mathrm{M}}$ in the Ussing chamber bath. Concentrations were increased sequentially in the chamber bath, accounting for the residual drug concentration from the previous drug application. Tissues were allowed to reach a steady-state prior to the application of the next administered concentration. A separate group of tissues $(n=4)$ were given an equivalent volume of DMSO (vehicle) at each time point of flupirtine administration, to account for time/vehicle-dependent reduction in ISC $_{\mathrm{SC}}$ when calculating \% inhibition values. The total volume of DMSO added during the experiment was 13 ! $\mathrm{L}(0.26 \%$ of bath volume). The average change in 
$\mathrm{I}_{\mathrm{SC}}$ after each DMSO "dose" was subtracted from the response at each corresponding concentration of flupirtine. This was done to avoid arbitrary pairing between vehicle- and flupirtine-treated tissues. \% inhibition was calculated using the following equation:

$$
\% \text { inhibition of basal } \mathrm{I}_{\mathrm{SC}}=\left(\Delta \mathrm{I}_{\mathrm{SC}} \mathrm{FLU}_{[\mathrm{X}]^{-}}-\Delta \mathrm{I}_{\mathrm{SC}} \mathrm{DMSO}\right) / \text { basal } \mathrm{I}_{\mathrm{SC}} \cdot 100
$$

$\Delta \mathrm{I}_{\mathrm{SC}} \mathrm{FLU}_{[\mathrm{X}]}$ and $\Delta \mathrm{I}_{\mathrm{SC}} \mathrm{DMSO}$ refer to the difference between basal $\mathrm{I}_{\mathrm{SC}}$ and residual $\mathrm{I}_{\mathrm{SC}}$ after flupirtine or DMSO application, respectively, for each concentration (X). \% inhibition values were fitted to a curve using non-linear regression ( $\log _{[\mathrm{drug}]}$ vs response; variable slope) in GraphPad Prism 8.0 software with the equation $\mathrm{Y}=$ Bottom $+($ Top-Bottom $) /\left(1+10^{\wedge}((\operatorname{LogIC} 50-\mathrm{X}) *\right.$ HillSlope $\left.)\right)$.

\section{Statistical analysis}

All presented data represent mean \pm standard error (SEM). $n$ values ranged from 4-5, depending on the experiment and are given in each of the figure legends. Statistical comparison between two treatment groups was done with an unpaired Student's t-test, with Bonferroni correction for multiple t-tests, using GraphPad Prism 8.0 software (San Diego, CA). $p<0.05$ was used for the determination of statistical significance $(*)$.

\section{Chemicals and stock solutions}

Indomethacin and carbamoylcholine (carbachol; $\mathrm{CCH}$ ) were purchased from Sigma Aldrich (St. Louis, MO). Veratridine (VER) was purchased from Alomone Labs (Jerusalem, Israel). Flupirtine (FLU), Tetrodotoxin (TTX), Vasoactive Intestinal polypeptide (VIP), Nicotine (NICO), Hexamethonium (HEX) and ICA 110381 were purchased from Tocris Pharmaceuticals (Minneapolis, MN). All drugs were prepared as 1000x stocks and diluted to their working concentrations in the Ussing chamber bath, with the exception of FLU for concentration response experiments (described above). TTX, CCH, VIP, NICO and HEX were dissolved in water. 
Indomethacin was dissolved in ethanol. VER, FLU and ICA 110381 were dissolved in DMSO. When added to the Ussing chamber bath, the DMSO concentration was increased by $0.1 \%$ after each drug administration ( $5 \mu \mathrm{L}$ of DMSO into $5 \mathrm{~mL}$ bath).

\section{RESULTS}

Flupirtine, a $\mathrm{K}_{v} 7$ channel activator, inhibits basal $\mathrm{Cl}^{\top}$ secretion in mouse distal colon.

Initial experiments were conducted in order to assess the effect of a non-selective $\mathrm{K}_{\mathrm{V}} 7$ activator, flupirtine (FLU), on basal $\mathrm{I}_{\mathrm{SC}}$ in ENS-intact mouse distal colon. A concentration of 100 $\mu \mathrm{M}$ was chosen based on the previous studies involving ex vivo GI tissues $(1,28)$. Application of $100 \mu \mathrm{M}$ flupirtine to the serosal bath inhibited $\mathrm{I}_{\mathrm{SC}}$ significantly, compared to vehicle (DMSO) control (FLU vs DMSO: $\Delta \mathrm{I}_{\mathrm{SC}}=-87.5 \pm 10.4$ vs $-14.8 \pm 4.2 \mu \mathrm{A} / \mathrm{cm}^{2} ; p<0.01$ ) (Fig. 1A-C). Inhibition of $I_{\mathrm{SC}}$ was not the result of impaired tissue integrity, as trans-epithelial conductance was also reduced after flupirtine administration $\left(\Delta \mathrm{G}_{\mathrm{t}}=-0.6 \pm 0.1 \mathrm{mS} / \mathrm{cm}^{2}\right)$. A residual steady-state $\mathrm{I}_{\mathrm{SC}}$ of $57.0 \pm 11.9 \mu \mathrm{A} / \mathrm{cm}^{2}$ persisted post-treatment, corresponding to $\mathrm{Cl}^{-}$secretion that was not inhibited by flupirtine.

The concentration-dependence of $\mathrm{I}_{\mathrm{SC}}$ inhibition in response to sequentially increasing concentrations of flupirtine in the basolateral bath was determined next (Fig. 1D). An apparent half-maximal inhibitory concentration $\left(\mathrm{IC}_{50}\right.$ ) for flupirtine was calculated to be $5.8 \mu \mathrm{M}$ by fitting a non-linear regression to normalized data (\% inhibition of basal $\left.\mathrm{I}_{\mathrm{SC}}\right)$ obtained from 4 separate animals. In the experiments to follow, flupirtine was administered at $100 \mu \mathrm{M}$, as this concentration produced a consistent maximal inhibition of ISC. $_{\text {SC }}$ 
Flupirtine and tetrodotoxin produce similar inhibitory effect on basal $\mathrm{Cl}^{\prime}$ secretion in mouse distal colon.

If the inhibitory effect of flupirtine on $\mathrm{ISC}_{\mathrm{SC}}$ is attributable to its hyperpolarizing effects on ENS neurons via activation of $\mathrm{K}_{\mathrm{V}} 7$ channels, then subsequent addition of an alternative ENStargeted inhibitor following pre-treatment with flupirtine should produce minimal or no further effect. Tetrodotoxin (TTX; a Nav channel blocker) is routinely used for the purpose of diminishing ENS-stimulated secretion in intestinal preparations (13). Because $\mathrm{Na}_{\mathrm{V}}$ channels produce the depolarizing current necessary to generate an action potential, inhibition by tetrodotoxin renders the cell unable to propagate an action potential and thus, neurotransmitter cannot be released (6). In contrast, flupirtine induces a hyperpolarizing $\mathrm{K}^{+}$current, inhibiting action potential generation through the mechanism that is distinct from that of tetrodotoxin.

Flupirtine $(100 \mu \mathrm{M})$ and tetrodotoxin $(1 \mu \mathrm{M})$ both inhibited basal $\mathrm{ISC}_{\mathrm{SC}}$ in mouse colon when administered to serosal bath (Fig. 2 A-B). $G_{t}$ was also reduced by tetrodotoxin ( $6 G_{t}=-0.4 \pm 0.1$ $\mathrm{mS} / \mathrm{cm}^{2}$ ). Following treatment with either flupirtine or tetrodotoxin, subsequent addition of the other drug produced only minimal further inhibition. Flupirtine-mediated $\mathrm{I}_{\mathrm{SC}}$ inhibition was significantly attenuated by pre-treatment with tetrodotoxin (FLU vs TTX/FLU: $\Delta \mathrm{I}_{\mathrm{SC}}=-75.8 \pm$ 13.3 vs $\left.-9.3 \pm 2.9 \mu \mathrm{A} / \mathrm{cm}^{2} ; p<0.01\right)$ (Fig. $2 \mathrm{C}$ ). Conversely, tetrodotoxin-mediated $\mathrm{I}_{\mathrm{SC}}$ inhibition was significantly attenuated by pre-treatment with flupirtine (TTX vs FLU/TTX: $\Delta \mathrm{I}_{\mathrm{SC}}=-65.3 \pm$ 17.9 vs $-3.2 \pm 0.7 \mu \mathrm{A} / \mathrm{cm}^{2} ; p<0.01$ ) (Fig. 2D). Residual $\mathrm{I}_{\mathrm{SC}}$ values following either flupirtine or tetrodotoxin were not significantly different $\left(\mathrm{I}_{\mathrm{SC}}=48.3 \pm 7.4\right.$ vs $57.3 \pm 4.7 \mu \mathrm{A} / \mathrm{cm}^{2}$, respectively; $p=0.29)$ and did not appear to be dependent on the size of the $\Delta \mathrm{I}_{\mathrm{SC}}$ for either flupirtine or tetrodotoxin. These findings indicate that flupirtine-induced inhibition of I IS likely occurs via a tetrodotoxin-sensitive pathway. 
Flupirtine modulates the stimulatory response to veratridine in mouse distal colon.

Following the observation that flupirtine completely negated tetrodotoxin-induced ISC inhibition, the effect of flupirtine on the $\mathrm{I}_{\mathrm{SC}}$ response to $\mathrm{Na}_{\mathrm{V}}$ channel activation was determined next. Veratridine (VER) is a selective $\mathrm{Na}_{\mathrm{V}}$ channel opener that induces action potential firing in neurons and triggers subsequent stimulation of the colonic epithelium, generating a secretory response (21). If flupirtine hyperpolarizes ENS neuronal membrane potential via $\mathrm{K}_{\mathrm{V}} 7$ channel activation, then the secretory response to depolarization by veratridine may be diminished or delayed in the presence of flupirtine.

Addition of veratridine to the serosal bath induced a rapid and transient increase in $\mathrm{I}_{\mathrm{SC}}$ (Fig. 3A), consistent with previous reports (21). Tissues pre-treated with flupirtine had an altered $\mathrm{I}_{\mathrm{SC}}$ response to veratridine (Fig. 3B), although the magnitude of veratridine-induced secretion was not significantly reduced in flupirtine-treated tissues (VER vs FLU/VER: $\Delta \mathrm{I}_{\mathrm{SC}}=66.2 \pm 11.4$ vs $41.8 \pm$ $8.0 \mu \mathrm{A} / \mathrm{cm}^{2} ; p=0.085$ ) (Fig. 3C). However, the time necessary to reach half-maximal activation of Isc was dramatically increased in flupirtine-treated compared to untreated tissues (with vs without FLU: $4.1 \pm 0.7$ vs $1.3 \pm 0.2$ minutes; $p<0.01$ ) (Fig. 3D). The observed delay in the response to veratridine may be attributable to flupirtine-induced hyper-polarizing $\mathrm{K}_{\mathrm{V}} 7$ current opposing the depolarization induced by $\mathrm{Na}_{\mathrm{V}}$ channel opening, resulting in a longer time period necessary to reach full stimulatory effect. This observation further suggests that flupirtine acts upon cell types which express $\mathrm{Na}_{\mathrm{V}}$ channels (i.e. neurons).

Nicotine- and hexamethonium-induced $I_{S C}$ responses are attenuated by flupirtine in mouse distal colon. 
Nicotinic acetylcholine receptors (nAChRs) are found within the ENS, where they contribute to ganglionic transmission and resultant stimulation of epithelial secretion $(19,23)$. As they are primarily expressed by neurons, pharmacological targeting of nAChRs provides another avenue for assessing the effect of flupirtine on stimulatory or inhibitory events that are largely (if not entirely) dependent on the ENS. Experiments were therefore conducted to determine if flupirtine interferes with the response to either activation or inhibition of nAChRs.

Serosal nicotine (NICO; nAChR agonist) application induced a robust and transient increase in $\mathrm{I}_{\mathrm{SC}}$, which was completely abolished by pre-treatment with flupirtine (NICO vs FLU/NICO: $\Delta \mathrm{I}_{\mathrm{SC}}=93.0 \pm 18.9$ vs $0.3 \pm 1.1 \mu \mathrm{A} / \mathrm{cm}^{2} ; p<0.01$ (Figs. $4 \mathrm{~A}-4 \mathrm{C}$ ). The response to nicotine in the presence of flupirtine was not significantly different from zero $(p=0.82)$. Hexamethonium (HEX; nAChR antagonist) administration partially reduced basal $\mathrm{I}_{\mathrm{SC}}$, while its inhibitory effect was negated by pre-treatment with flupirtine (HEX vs FLU/HEX: $\Delta \mathrm{I}_{\mathrm{SC}}=-51.2 \pm$ $\left.6.1 \mathrm{vs}-2.3 \pm 0.6 \mu \mathrm{A} / \mathrm{cm}^{2} ; p<0.01\right)$ (Figs. 5A - 5C). Hexamethonium reduced $\mathrm{G}_{\mathrm{t}}\left(\underset{4}{4} \mathrm{G}_{\mathrm{t}}=-0.5 \pm 0.1\right.$ $\mathrm{mS} / \mathrm{cm}^{2}$ ) and pre-treatment with hexamethonium also significantly reduced $\mathrm{I}_{\mathrm{SC}}$ inhibition in response to flupirtine (FLU vs HEX/FLU: $\Delta \mathrm{I}_{\mathrm{SC}}=-82.6 \pm 7.0$ vs $-52.0 \pm 4.3 \mathrm{vs} \mu \mathrm{A} / \mathrm{cm}^{2} ; p<0.01$ ) (Fig. 5D), suggesting that at least a portion of the basal $\mathrm{I}_{\mathrm{SC}}$ inhibited by flupirtine is mediated by nicotinic transmission. The remaining hexamethonium-insensitive, flupirtine-sensitive $\mathrm{I}_{\mathrm{SC}}$ may be attributable to basal neurogenic secretion that is not dependent on nicotinic ganglionic transmission (e.g. serotonin- or ATP-dependent mechanisms). Nonetheless, as nAChRs have a well-established role in enteric neuronal communication, flupirtine interference with both nAChR stimulation and inhibition lends further support to flupirtine having a site of action within the ENS. 
Epithelium-targeted secretagogues, VIP and carbachol, stimulate $\mathrm{Cl}^{-}$secretion in the presence and absence of flupirtine in mouse distal colon.

If flupirtine inhibits stimulatory neurotransmitter release by hyperpolarizing ENS neurons, then application of exogenous epithelium-targeted secretagogues should still elicit a response in flupirtine-treated tissues. Experiments were thus performed utilizing secretagogues which target the epithelium directly by acting on basolateral receptors for endogenous neurotransmitters: vasoactive intestinal polypeptide (VIP), the endogenous ligand of VPAC receptors and carbachol $(\mathrm{CCH})$, a non-selective nicotinic and muscarinic acetylcholine receptor (mAChR) agonist. VIP and carbachol were selected because VIP-ergic and cholinergic neurons were recently demonstrated to be two of the major populations of secretomotor neurons present in mouse colon (16). VIP and carbachol also stimulate $\mathrm{Cl}^{-}$secretion via cAMP- and $\mathrm{Ca}^{2+}$-dependent pathways, respectively, representing the two primary pathways involved in epithelial $\mathrm{Cl}^{-}$secretion.

As expected, VIP and carbachol both elicited a robust secretory response (Fig. 6 A,D). Pretreatment with flupirtine did not significantly inhibit the response to VIP (VIP vs. FLU/VIP: $\Delta \mathrm{I}_{\text {SC }}$ $=88.2 \pm 21.9$ vs $\left.61.1 \pm 17.0 \mu \mathrm{A} / \mathrm{cm}^{2} ; p=0.36\right)($ Fig. 6 B-C). Flupirtine pre-treatment did partially, but significantly inhibit the response to carbachol $\left(\mathrm{CCH}\right.$ vs FLU/CCH: $\Delta \mathrm{I}_{\mathrm{SC}}=79.2 \pm 9.6$ vs 44.0 $\pm 3.3 \mu \mathrm{A} / \mathrm{cm}^{2} ; p<0.01$ ) (Fig. $\left.6 \mathrm{E}-\mathrm{F}\right)$, but did not abolish the response altogether, as was the case for nicotine (Fig. 4). This may be due to carbachol acting both on ENS-localized nAChRs and epithelial/neuronal mAChRs, whereas nicotine acts solely upon nAChRs. It should be mentioned that the use of a selective muscarinic receptor agonist would provide no significant advantage over a general cholinergic agonist (such as carbachol), as muscarinic receptors are expressed by ENS neurons as well as on the epithelium, making it impossible to completely distinguish between the two sites of action. Ultimately, the response to either VIP or carbachol in the presence of flupirtine 
further suggests that flupirtine inhibits $\mathrm{I}_{\mathrm{SC}}$ via an ENS-dependent mechanism, and not through effects on the epithelium directly.

Flupirtine does not inhibit $\mathrm{Cl}^{-}$secretion in epithelial T84 monolayers.

T84 monolayers have been used as a model to study the colonic epithelium for several decades $(57,58)$. The T84 cell line was originally isolated from a lung metastasis of colorectal adenocarcinoma, and consists of a homogenous population of secretory epithelium (15). Therefore, any drug acting specifically on ENS neurons should not elicit a response in T84 cells. When flupirtine was added to the serosal (basolateral) bath of T84 monolayers mounted in the Ussing chamber, no measurable $\mathrm{I}_{\mathrm{SC}}$ response was produced (Fig. 7A). Forskolin (FSK) was used to stimulate $\mathrm{I}_{\mathrm{SC}}$ (via adenylate cyclase activation; cAMP-induced $\mathrm{Cl}^{-}$secretion) to confirm that the monolayers were properly developed and capable of generating a secretory response. Application of basolateral flupirtine following forskolin stimulation produced only a minor change in $\mathrm{ISC}_{\mathrm{SC}}(-0.8$ $\left.\pm 0.2 \mu \mathrm{A} / \mathrm{cm}^{2}\right)$ (Fig. 7B), although statistically greater than zero $(p<0.01)$. The response to forskolin stimulation was not significantly altered by pre-treatment with flupirtine (FSK vs FLU/FSK: $\Delta \mathrm{I}_{\mathrm{SC}}=39.3 \pm 4.2 \mathrm{vs} 32.4 \pm 1.9 \mu \mathrm{A} / \mathrm{cm}^{2} ; p=0.18$ ) (Fig. 7C). The absence of an effect on either basal or forskolin-stimulated $\mathrm{I}_{\mathrm{SC}}$ when applied to T84 cells provides additional functional support for flupirtine having a non-epithelial site of action.

Flupirtine inhibits basal and stimulated $\mathrm{Cl}$ secretion in longitudinal and circular smooth muscle stripped mouse distal colon.

Neurons in the submucosal plexus of the ENS are generally associated with epithelial secretion, while neurons of the myenteric plexus are considered to regulate motility (20). To 
determine if basal $I_{S C}$ inhibition by flupirtine was attributable to effects on the submucosal plexus specifically, flupirtine was administered to colonic tissues stripped of longitudinal and circular smooth muscle, and thus lacking the myenteric plexus. Representative images of H\&E-stained unstripped (i.e. full-thickness) (Fig. 8A-B) and seromuscular-stripped (Fig. 8C-D) mouse distal colon are shown to illustrate the depth of seromuscular stripping used in these experiments. It should be noted that while these representative images depict sectioned tissues that have been folded over, tissues used for Ussing chamber experiments were flattened when mounted on the chamber sliders. Representative recordings demonstrating flupirtine-induced $\mathrm{I}_{\mathrm{SC}}$ inhibition are shown for unstripped (Fig. 8E) and seromuscular-stripped tissues (Fig. 8F).

Basal $\mathrm{I}_{\mathrm{SC}}$ inhibition by flupirtine was not different between unstripped and stripped colon in terms of magnitude (stripped vs unstripped: $\Delta \mathrm{I}_{\mathrm{SC}}=-70.4 \pm 13.4 \mathrm{vs}-73.6 \pm 7.4 \mu \mathrm{A} / \mathrm{cm}^{2} ; p=$ 0.802) (Fig. 8G), or the time necessary to reach half-maximal inhibition (stripped vs unstripped: $3.5 \pm 0.6$ vs $2.5 \pm 0.4$ minutes; $p=0.14)($ Fig. $8 \mathrm{H})$. Residual $\mathrm{I}_{\mathrm{SC}}$ after flupirtine treatment was also not significantly different between full-thickness and seromuscular-stripped tissues (stripped vs unstripped: $\mathrm{I}_{\mathrm{SC}}=73.3 \pm 11.0$ vs $\left.61.7 \pm 9.8 \mu \mathrm{A} / \mathrm{cm}^{2} ; p=0.37\right)$.

In addition, the secretory response to nicotine was abolished by flupirtine pre-treatment in stripped tissues (NICO vs FLU/NICO: $\Delta \mathrm{I}_{\mathrm{SC}}=72.1 \pm 21.9$ vs $0.8 \pm 2.3 \mu \mathrm{A} / \mathrm{cm}^{2} ; p<0.05$ ) (Fig. 9 A,C,E), similar to the previous experiments using intact tissues (Fig. 4). Hexamethonium added to the serosal bath also inhibited basal $\mathrm{I}_{\mathrm{SC}}$ in stripped tissues, but this effect was attenuated by pretreatment with flupirtine as well (HEX vs FLU/HEX: $\Delta \mathrm{I}_{\mathrm{SC}}=-51.4 \pm 11.4 \mathrm{vs}-11.9 \pm 4.7 \mu \mathrm{A} / \mathrm{cm}^{2}$; $p<0.05$ ) (Fig. 9 B,D,F). Additionally, flupirtine inhibited a portion of residual $\mathrm{I}_{\mathrm{SC}}$ in tissues treated with hexamethonium $\left(\Delta \mathrm{I}_{\mathrm{SC}}=-44.1 \pm 15.1 \mu \mathrm{A} / \mathrm{cm}^{2}\right)$ and this $\Delta \mathrm{I}_{\mathrm{SC}}$ value was significantly greater than $0.0(p=0.04)$. This suggests that flupirtine-mediated basal $\mathrm{I}_{\mathrm{SC}}$ inhibition is not dependent on 
the presence of the myenteric plexus. It is therefore plausible that $\mathrm{K}_{\mathrm{V}} 7$ channels within the submucosal plexus mediated the inhibition of basal $\mathrm{I}_{\mathrm{SC}}$ induced by flupirtine in the experiments described above.

$K_{V} 7.2$ and $K_{V} 7.3$ channel subtypes are expressed in the submucosal plexus of mouse distal colon.

Immunofluorescent labelling of $\mathrm{K}_{\mathrm{V}} 7.2$ and $\mathrm{K}_{\mathrm{V}} 7.3$ within the submucosa was performed, as these two channel subtypes together produce the "M" current and are widely known to regulate membrane potential in neurons. Tissues were labelled for either $\mathrm{K}_{\mathrm{V}} 7.2$ or $\mathrm{K}_{\mathrm{V}} 7.3$ in conjunction with microtubule-associated protein 2 (MAP2; a neuron-specific marker). Figure 10 illustrates that cells were co-labelled with $\mathrm{K}_{\mathrm{V}} 7.2$ and MAP2 (Fig. 10A) or $\mathrm{K}_{\mathrm{V}} 7.3$ and MAP2 (Fig. 10B), indicating that these two $\mathrm{K}_{\mathrm{V}} 7$ subtypes are localized within the submucosal ENS neurons in mouse distal colon. $\mathrm{K}_{\mathrm{V}} 7.2$ or $\mathrm{K}_{\mathrm{V}} 7.3$ was detected in the majority of MAP2-positive cells. $\mathrm{K}_{\mathrm{V}} 7.2$ and $\mathrm{K}_{\mathrm{V}} 7.3$ were also detected in cells not labelled with MAP2, possibly corresponding to glial or smooth muscle cells. Further investigation of non-neuronal expression of $\mathrm{K}_{\mathrm{V}} 7.2$ and $\mathrm{K}_{\mathrm{V}} 7.3$ was not pursued in this study, but may be of interest for future work.

\section{ICA 110381 inhibits basal $\mathrm{Cl}^{-}$secretion in mouse colon.}

Immunofluorescence studies indicated the presence of $K_{V} 7.2$ and $K_{V} 7.3$ proteins in submucosal neurons. Co-localization of $\mathrm{K}_{\mathrm{V}} 7.2$ and $\mathrm{K}_{\mathrm{V}} 7.3$ could not be determined by our immunofluorescence experiments because both primary antibodies were raised in rabbit, precluding the use of multiple fluorophore-conjugated secondary antibodies. However, the $\mathrm{K}_{\mathrm{V}} 7.2 / 3$ heterotetramer is known to play a vital role in the function of many neuronal cell populations in central and peripheral nervous systems. ICA 110381, a selective activator for 
$\mathrm{K}_{\mathrm{V}} 7.2 / 3$ heterotetramers (9) was used to determine if activation of $\mathrm{K}_{\mathrm{V}} 7.2 / 3$ channels would inhibit basal $\mathrm{I}_{\mathrm{SC}}$ comparably to non-selective $\mathrm{K}_{\mathrm{V}} 7$ activation by FLU. The reported $\mathrm{EC}_{50}$ values for ICA110381 activation for $\mathrm{K}_{\mathrm{V}} 7.1$ and $\mathrm{K}_{\mathrm{V}} 7.2 / 3$ activation are 15 and $0.38 \mathrm{mM}$, rescpetively (3), indicating the drug's specificity for $\mathrm{K}_{\mathrm{V}} 7.2 / 3$ heterotetramers. Addition of ICA 110381 to the serosal bath inhibited basal $\mathrm{I}_{\mathrm{SC}}$ (Fig. 11A). Figure 10B shows a representative response to flupirtine for comparison. Residual $\mathrm{I}_{\mathrm{SC}}$ following ICA 110381 administration was $42.8 \pm 9.2 \mu \mathrm{A} / \mathrm{cm}^{2}$. The magnitude of FLU-mediated ISC inhibition appears to be slightly greater compared to ICA 110381 , although this difference was not statistically significant (FLU vs ICA: $\Delta \mathrm{I}_{\mathrm{SC}}=-86.6 \pm 10.4$ vs. $60.0 \pm 9.4 \mu \mathrm{A} / \mathrm{cm}^{2} ; p=0.09$ ) (Fig. 11C). Inhibition of basal $\mathrm{I}_{\mathrm{SC}}$ by ICA 110381 indicates that $\mathrm{K}_{\mathrm{V}} 7.2 / 3$ heterotetramers may be expressed in ENS neurons, where their activation decreases epithelial $\mathrm{Cl}^{-}$secretion.

\section{DISCUSSION}

This study demonstrates that $\mathrm{K}_{\mathrm{V}} 7$ channel activation in submucosal enteric neurons inhibits basal $\mathrm{Cl}^{-}$secretion in mouse distal colon. This conclusion is supported by the following observations: 1) in Ussing chamber studies using unstripped mouse colon, flupirtine (FLU) inhibited basal ISC (Fig. 1). Flupirtine also abolished or attenuated the effects of ENS-targeted drugs (tetrodotoxin, veratridine, nicotine, hexamethonium) (Figs. 2-5). However, flupirtine did not abolish the effects of epithelium-targeted drugs, VIP or carbachol (Fig. 6). 2) Flupirtine had no effect on basal or stimulated ISC the epithelial T84 cell line, which lacks an ENS (Fig. 7), but inhibited basal $\mathrm{I}_{\mathrm{SC}}$ in seromuscular-stripped mouse colon, which lacks a myenteric plexus but contains submucosal neurons (Fig. 8). 3) $\mathrm{K}_{\mathrm{V}} 7.2$ and $\mathrm{K}_{\mathrm{V}} 7.3$ channel subunits were detected in 
submucosal neurons (Fig. 9), while the selective $\mathrm{K}_{\mathrm{V}} 7.2 / 3$ activator, ICA 110381, also inhibited basal $\mathrm{I}_{\mathrm{SC}}$ in mouse distal colon (Fig. 10).

Addition of flupirtine to the serosal bath inhibited basal ISC (i.e., $\mathrm{Cl}^{-}$secretion) in unstripped (full-thickness) mouse distal colon (Fig. 1A-C). The residual $\mathrm{I}_{\mathrm{SC}}$ after flupirtine treatment likely corresponds to $\mathrm{Cl}^{-}$secretion generated by the epithelium itself as part of its basal transport function. The identities of the epithelial channels and transporters which facilitate the observed $\mathrm{Cl}^{-}$secretion (basal or residual) were not investigated here, although existing literature suggests that the basolateral co-transporter, $\mathrm{NKCC} 1$, and apical CFTR channel are likely responsible (32). An $\mathrm{IC}_{50}$ for flupirtine was calculated to be $\sim 5.8 \mu \mathrm{M}$ (Fig. 1D). This value falls between the those reported for flupirtine in patch clamp experiments using rat hippocampal neurons $(0.6 \mu \mathrm{M})(25)$ and in motor activity experiments using human taenia coli $(29.9 \mu \mathrm{M})(1)$. Indeed, $\mathrm{IC}_{50}$ and $\mathrm{EC}_{50}$ values for drugs are generally higher in Ussing-style chamber experiments compared to other methods, owing to drug diffusion barriers, precipitation by $\mathrm{HCO}_{3}{ }^{-}$etc. (13), and the $\mathrm{IC}_{50}$ value of $5.8 \mu \mathrm{M}$ calculated here lies within the expected range.

The ability of flupirtine to completely attenuate the inhibitory effect of tetrodotoxin (Fig. 2) and significantly delay the stimulatory response to veratridine (Fig. 3) indicates that flupirtine mediates its effects through cell types which express $\mathrm{Na}_{\mathrm{V}}$ channels. Although the response to veratridine was not diminished significantly in terms of magnitude, a substantial delay in onset of secretion was observed. Interestingly, veratridine-induced $\mathrm{I}_{\mathrm{SC}}$ levels did not return to baseline in the presence of flupirtine, as they did in untreated tissues. The reason for this finding is unclear but may be the result of flupirtine (hyperpolarizing $\mathrm{K}_{\mathrm{V}} 7$ currents) preventing a tachyphylactic response to veratridine within ENS neurons, or to the subsequently-released neurotransmitters at the level of the epithelium. However, this interpretation is purely speculative. 
Another major finding of this study is that nicotine and hexamethonium produced no response in flupirtine-treated tissues (Figs. 4,5 and 9), further supporting the working model of an ENS site of action for flupirtine, as nicotinic receptor (nAChR) stimulation is a generally associated with neuron-to-neuron communication. There have been reports of $\mathrm{nAChR}$ subtype expression in colonic epithelial cells, but their function may be related to $\mathrm{Na}^{+} / \mathrm{K}^{+}$ATPase regulation (metabotropic) and not membrane de-polarization (ionotropic), as epithelial cells are not excitable in the classical sense (40). However, it is well-established that nAChR's play an important role in neurotransmission in the ENS $(19,23)$. Thus, the interference of nicotinic transmission by flupirtine further supports an ENS site of action.

Vasoactive intestinal polypeptide (VIP) and carbachol both stimulate the colonic epithelium directly through $\mathrm{VPAC} 1$ and $\mathrm{M}_{3} \mathrm{R}$ receptors, respectively, which are expressed on the basolateral membrane of crypt cells $(5,27)$, and thus are not dependent on ENS neurons to mediate their effects. As predicted, both agents generated a response in the presence and absence of flupirtine (Fig. 6). Pre-treatment with flupirtine did not reduce the response to VIP, but did partially reduce the response to carbachol. This finding is still consistent with the working hypothesis because carbachol stimulates both nAChRs (neuronal) and mAChRs (neuronal and epithelial). The decrease in carbachol-stimulated $\mathrm{I}_{\mathrm{SC}}$ in flupirtine-treated tissues is expected because $\mathrm{nAChR}$ activation by carbachol had presumably been negated by the hyperpolarizing effect of flupirtine, while stimulation of epithelial $\mathrm{M}_{3} \mathrm{R}$ remains intact. Enteric neurons also express VPAC receptors and VIP signaling is important for neuron-glia communication as well (17). However, under these conditions, any inter-neuronal or glial component to VIP-stimulated $\mathrm{Cl}^{-}$secretion appears to be unaffected by pre-treatment with flupirtine. 
Previous reports have identified $\mathrm{K}_{\mathrm{V}} 7$ channel proteins in the myenteric plexus, as well as within GI smooth muscle $(28,49)$. Other $\mathrm{K}_{\mathrm{V}} 7$ channel activators (e.g. retigabine) have been shown to reduce colonic motility and smooth muscle contraction - functions generally associated with the myenteric plexus and the smooth muscle itself, which also expresses $\mathrm{K}_{V} 7$ channels (28). Here we find evidence of $K_{V} 7$ channel subunits, $K_{V} 7.2$ and $K_{V} 7.3$, in submucosal neurons (Fig. 10). Additionally, a selective $\mathrm{K}_{\mathrm{V}} 7.2 / 3$ activator, ICA 110381, also inhibited $\mathrm{I}_{\mathrm{SC}}$ in mouse distal colon (Fig. 11). $\mathrm{K}_{\mathrm{V}} 7.2 / 3$ channels are known to regulate the function of other neuronal cell types (51). Our findings suggest they may modulate ENS-driven $\mathrm{Cl}^{-}$secretion in the colon as well. This, coupled with previous reports showing efficacy of $\mathrm{K}_{\mathrm{V}} 7$ channel activators in attenuating GIlocalized pain (49), as well as reducing smooth muscle contractile activity (28), make $\mathrm{K}_{\mathrm{V}} 7$ channels an attractive target for drug development. Future studies should be directed at characterizing the expression of specific $\mathrm{K}_{\mathrm{V}} 7$ proteins and their homo/heterotetrameric assembly within enteric neurons, as well as identifying whether flupirtine/ICA-sensitive channels are confined to a particular neuronal subpopulation.

Another member of the $\mathrm{K}_{\mathrm{V}} 7$ family, $\mathrm{K}_{\mathrm{V}} 7.1$, has been well-characterized in terms of colonic epithelial cell function. $\mathrm{K}_{\mathrm{V}} 7.1$ is expressed on the basolateral membrane of crypt epithelial cells, where its activation supports sustained apical $\mathrm{Cl}^{-}$secretion by providing an electrochemical driving force for apical $\mathrm{Cl}^{-}$exit $(31,42)$. While flupirtine is a "non-selective" $\mathrm{K}_{\mathrm{V}} 7$ activator, and may therefore act upon epithelium-localized $\mathrm{K}_{\mathrm{V}} 7.1$, in theory this would increase $\mathrm{Cl}^{-}$secretion by creating an electrical gradient favoring $\mathrm{Cl}^{-}$efflux. In this study, the opposite effect was observed, suggesting that flupirtine either 1) does not stimulate $\mathrm{Cl}^{-}$secretion by activating basolateral $\mathrm{K}_{V} 7.1$, or 2) any stimulatory effect is overshadowed by the inhibitory effects on ENS-driven secretion as described above. Moreover, flupirtine had no effect on baseline $\mathrm{Cl}^{-}$secretion and did not enhance 
or alter the effect of forskolin stimulation in T84 cells (Fig. 7). T84 cells therefore may not express flupirtine-sensitive $\mathrm{K}_{\mathrm{V}} 7$ channels, or their activation does not alter basal or forskolin-stimulated secretion under these experimental conditions.

Interestingly, the anti-convulsant, retigabine - a structural analogue of flupirtine - was found to have no effect on $K_{V} 7.1$ channels in cardiac cells, and preferentially activates $K_{V} 7.2$ Kv7.5 (44). Unfortunately, there is little data available regarding the sensitivity of each $\mathrm{K}_{\mathrm{V}} 7$ family subunit $\left(\mathrm{K}_{\mathrm{V}} 7.1-\mathrm{Kv} 7.5\right)$ to flupirtine, particularly in peripheral neural networks, such as the ENS. However, it is possible that $\mathrm{K}_{\mathrm{V}} 7.1$ is less sensitive or insensitive to flupirtine as well, given the similar structural and pharmacological profiles of the two drugs $(44,33) . \mathrm{K}_{\mathrm{V}} 7.1$ is also known to associate with a regulatory or-subunit, KCNE3, in intestinal crypt cells $(7,37)$. This association may also have some bearing on the apparent insensitivity of epithelial $\mathrm{K}_{\mathrm{V}} 7.1$ to flupirtine in the experiments described here.

Flupirtine is considered to be a non-steroidal, non-opioid analgesic (35). Investigation into its analgesic properties has revealed several possible mechanisms and sites of action besides direct activation of $\mathrm{K}_{\mathrm{V}} 7$ channels. These possible effects include indirect antagonism of $N$-methyl- $D-$ aspartate (NMDA) receptors through voltage-dependent $\mathrm{Mg}^{2+}$ block resulting from $\mathrm{K}_{\mathrm{V}}$-mediated hyperpolarizing current (29), as well as direct activation of G-protein-activated inward rectifying $\mathrm{K}^{+}$(GIRK) channels (26) and $\gamma$-aminobutyric acid (GABA) receptors (35). As the concentration of flupirtine used in the present study was relatively high $(100 \mu \mathrm{M})$, the possible involvement of alternative mechanisms, such as those listed above, cannot be excluded. Nonetheless, if flupirtine has a suppressive effect on ENS neuronal excitability which is partially attributable to one or more of these alternative mechanisms, the end result of reduced stimulatory input to the epithelium would be effectively equivalent in terms of measured secretory activity within the epithelium. A 
detailed pharmacoanalysis of these "off-target" effects, specifically in ENS neurons, should be conducted in future studies to determine the exact nature of the flupirtine's apparent suppressive effects on neurogenic secretion.

Previous work has demonstrated that several gastrointestinal disorders, including IBS-D, result in an overall increase in the excitability of ENS neurons $(24,38,41,48)$. However, current clinical options are limited for these pathologies, with many observed adverse effects $(18,47)$. Flupirtine had been available for nearly three decades in several European countries for the treatment of chronic pain, as well as neurological disorders such as epilepsy, before being recently removed from the market due to issues of hepatotoxicity $(44,35,45)$. Additionally, flupirtine has been associated with a wide variety of generalized GI-related side effects, including constipation, diarrhea, abdominal pain and nausea (55). To our knowledge, there has not been an assessment to determine whether patients with pre-existing GI disorders (such as IBS) reported any of these side effects differentially (i.e. whether they were more or less likely to experience constipation or diarrhea, etc.). However, given that flupirtine is generally well-tolerated, the use of $\mathrm{K}_{\mathrm{V}} 7$ channel activators (whether flupirtine, ICA-110381 or a novel related compound) may prove to be beneficial in treating patients that have been unresponsive to traditional treatments for IBS-D, and these possibilities should be explored in the future.

In summary, our results demonstrate that activation of $\mathrm{K}_{V} 7$ channels significantly inhibits epithelial $\mathrm{Cl}^{-}$secretion in mouse distal colon. This effect is likely mediated through $\mathrm{K}_{\mathrm{V}} 7$ channels located within enteric neurons of the submucosal plexus, which decrease neuronal excitability and prevent stimulatory input to the epithelium. This discovery may have clinical significance with respect to future development of therapeutic agents to reduce ENS hyper-excitability in gastrointestinal pathologies, such as IBS-D. 


\section{ACKNOWLEDGEMENTS}

This study was supported by the National Institute of Health NIDDK R01DK104791 and DK1 12085 grants to VMR and P20GM103434, which supports WVU Microscpe Imaging Facility. 


\section{REFERENCES}

1. Adduci A, Martire M, Taglialatela M, et al. Expression and motor functional roles of voltage-dependent type $7 \mathrm{~K}(+)$ channels in the human taenia coli. Eur J Pharmacol. 2013;721(1-3):12-20. doi:10.1016/j.ejphar.2013.09.061

2. Alzamora R, O'Mahony F, Harvey BJ. Estrogen inhibits chloride secretion caused by cholera and Escherichia coli enterotoxins in female rat distal colon. Steroids. 2011;76(9):867-876. doi:10.1016/j.steroids.2011.04.016

3. Amato G, Roeloffs R, Rigdon GC, et al. N-Pyridyl and Pyrimidine Benzamides as KCNQ2/Q3 Potassium Channel Openers for the Treatment of Epilepsy. ACS Med Chem Lett. 2011;2(6):481-484. doi:10.1021/ml200053x

4. Anderson UA, Carson C, McCloskey KD. KCNQ currents and their contribution to resting membrane potential and the excitability of interstitial cells of Cajal from the guinea pig bladder. J Urol. 2009;182(1):330-336. doi:10.1016/j.juro.2009.02.108

5. Bachmann O, Reichelt D, Tuo B, Manns MP, Seidler U. Carbachol increases Na+-HCO3cotransport activity in murine colonic crypts in a M3-, Ca2+/calmodulin-, and PKCdependent manner. Am J Physiol Gastrointest Liver Physiol. 2006;291(4):G650-7. doi:10.1152/ajpgi.00376.2005

6. Bane V, Lehane M, Dikshit M, O'Riordan A, Furey A. Tetrodotoxin: chemistry, toxicity, source, distribution and detection. Toxins (Basel). 2014;6(2):693-755. doi:10.3390/toxins6020693

7. Barro-Soria R, Ramentol R, Liin SI, Perez ME, Kass RS, Larsson HP. KCNE1 and KCNE3 modulate KCNQ1 channels by affecting different gating transitions. Proc Natl Acad Sci U S A. 2017;114(35):E7367-E7376. doi:10.1073/pnas.1710335114

8. Bi Y, Chen H, Su J, Cao X, Bian X, Wang K. Visceral hyperalgesia induced by forebrainspecific suppression of native Kv7/KCNQ/M-current in mice. Mol Pain. 2011;7:84. doi:10.1186/1744-8069-7-84

9. Boehlen A, Schwake M, Dost R, et al. The new KCNQ2 activator 4-Chlor-N-(6-chlorpyridin-3-yl)-benzamid displays anticonvulsant potential. Br J Pharmacol. 2013;168(5):1182-1200. doi:10.1111/bph.12065

10. Brown DA, Passmore GM. Neural KCNQ (Kv7) channels. Br J Pharmacol. 2009;156(8):1185-1195. doi:10.1111/j.1476-5381.2009.00111.x

11. Camilleri M. Intestinal secretory mechanisms in irritable bowel syndrome-diarrhea. Clin Gastroenterol Hepatol Off Clin Pract J Am Gastroenterol Assoc. 2015;13(6):1051-1052. doi:10.1016/j.cgh.2014.07.020

12. Cangemi DJ, Lacy BE. Management of irritable bowel syndrome with diarrhea: a review of nonpharmacological and pharmacological interventions. Therap Adv Gastroenterol. 2019;12:1756284819878950. doi:10.1177/1756284819878950

13. Clarke LL. A guide to Ussing chamber studies of mouse intestine. AJP Gastrointest Liver Physiol. 2009;296(6):G1151-G1166. doi:10.1152/ajpgi.90649.2008

14. Condliffe SB, Doolan CM, Harvey BJ. 17beta-oestradiol acutely regulates Cl- secretion in rat distal colonic epithelium. J Physiol. 2001;530(Pt 1):47-54. doi:10.1111/j.1469- 
$7793.2001 .0047 \mathrm{~m} . \mathrm{x}$

15. Dharmsathaphorn K, McRoberts JA, Mandel KG, Tisdale LD, Masui H. A human colonic tumor cell line that maintains vectorial electrolyte transport. Am J Physiol. 1984;246(2 Pt 1):G204-8. doi:10.1152/ajpgi.1984.246.2.G204

16. Foong JPP, Tough IR, Cox HM, Bornstein JC. Properties of cholinergic and noncholinergic submucosal neurons along the mouse colon. J Physiol. 2014;592(4):777-793. doi:10.1113/jphysiol.2013.265686

17. Fung C, Boesmans W, Cirillo C, Foong JPP, Bornstein JC, Vanden Berghe P. VPAC Receptor Subtypes Tune Purinergic Neuron-to-Glia Communication in the Murine Submucosal Plexus. Front Cell Neurosci. 2017;11:118. doi:10.3389/fncel.2017.00118

18. Gallo-Torres H, Brinker A, Avigan M. Alosetron: ischemic colitis and serious complications of constipation. Am J Gastroenterol. 2006;101(5):1080-1083. doi:10.1111/j.1572-0241.2006.00650.x

19. Garza A, Huang LZ, Son J-H, Winzer-Serhan UH. Expression of nicotinic acetylcholine receptors and subunit messenger RNAs in the enteric nervous system of the neonatal rat. Neuroscience. 2009;158(4):1521-1529. doi:10.1016/j.neuroscience.2008.11.027

20. Gershon MD. Nerves, reflexes, and the enteric nervous system: pathogenesis of the irritable bowel syndrome. J Clin Gastroenterol. 2005;39(5 Suppl 3):S184-93. doi:10.1097/01.mcg.0000156403.37240.30

21. Grubišić V, Gulbransen BD. Enteric glial activity regulates secretomotor function in the mouse colon but does not acutely affect gut permeability. J Physiol. 2017;595(11):34093424. doi:10.1113/JP273492

22. Gunthorpe MJ, Large CH, Sankar R. The mechanism of action of retigabine (ezogabine), a first-in-class $\mathrm{K}+$ channel opener for the treatment of epilepsy. Epilepsia. 2012;53(3):412-424. doi:10.1111/j.1528-1167.2011.03365.x

23. Gwynne RM, Bornstein JC. Synaptic transmission at functionally identified synapses in the enteric nervous system: roles for both ionotropic and metabotropic receptors. Curr Neuropharmacol. 2007;5(1):1-17. doi:10.2174/157015907780077141

24. Houghton LA, Foster JM, Whorwell PJ. Alosetron, a 5-HT3 receptor antagonist, delays colonic transit in patients with irritable bowel syndrome and healthy volunteers. Aliment Pharmacol Ther. 2000;14(6):775-782. doi:10.1046/j.1365-2036.2000.00762.x

25. Ipavec V, Martire M, Barrese V, Taglialatela M, Currò D. KV7 channels regulate muscle tone and nonadrenergic noncholinergic relaxation of the rat gastric fundus. Pharmacol Res. 2011;64(4):397-409. doi:10.1016/j.phrs.2011.06.016

26. Jakob R, Krieglstein J. Influence of flupirtine on a G-protein coupled inwardly rectifying potassium current in hippocampal neurones. Br J Pharmacol. 1997;122(7):1333-1338. doi:10.1038/sj.bjp.0701519

27. Jayawardena D, Guzman G, Gill RK, Alrefai WA, Onyuksel H, Dudeja PK. Expression and localization of VPAC1, the major receptor of vasoactive intestinal peptide along the length of the intestine. Am J Physiol Gastrointest Liver Physiol. 2017;313(1):G16-G25. doi:10.1152/ajpgi.00081.2017 
28. Jepps TA, Greenwood IA, Moffatt JD, Sanders KM, Ohya S. Molecular and functional characterization of $\mathrm{Kv} 7 \mathrm{~K}+$ channel in murine gastrointestinal smooth muscles. Am J Physiol Gastrointest Liver Physiol. 2009;297(1):G107-15. doi:10.1152/ajpgi.00057.2009

29. Kornhuber J, Bleich S, Wiltfang J, Maler M, Parsons CG. Flupirtine shows functional NMDA receptor antagonism by enhancing $\mathrm{Mg} 2+$ block via activation of voltage independent potassium channels. J Neural Transm. 1999;106(9-10):857-867. doi:10.1007/s007020050206

30. Krause R, Ameen V, Gordon SH, et al. A randomized, double-blind, placebo-controlled study to assess efficacy and safety of $0.5 \mathrm{mg}$ and $1 \mathrm{mg}$ alosetron in women with severe diarrhea-predominant IBS. Am J Gastroenterol. 2007;102(8):1709-1719. doi:10.1111/j.1572-0241.2007.01282.x

31. Kunzelmann K, Hübner M, Schreiber R, et al. Cloning and function of the rat colonic epithelial K+ channel KVLQT1. J Membr Biol. 2001;179(2):155-164. doi:10.1007/s002320010045

32. Kunzelmann K, Mall M. Electrolyte transport in the mammalian colon: mechanisms and implications for disease. Physiol Rev. 2002;82(1):245-289. doi:10.1152/physrev.00026.2001

33. Kurata HT. Chemical regulation of Kv7 channels: Diverse scaffolds, sites, and mechanisms of action. J Gen Physiol. 2020;152(8):8-10. doi:10.1085/jgp.202012598

34. Kuwahara A, Radowicz-Cooke HJ. Epithelial transport in guinea-pig proximal colon: influence of enteric neurones. J Physiol. 1988;395:271-284. doi:10.1113/jphysiol.1988.sp016918

35. Lawson K. Pharmacology and clinical applications of flupirtine: Current and future options. World J Pharmacol. 2019;3192(1):1-13.

36. Lembo AJ, Lacy BE, Zuckerman MJ, et al. Eluxadoline for Irritable Bowel Syndrome with Diarrhea. N Engl J Med. 2016;374(3):242-253. doi:10.1056/NEJMoa1505180

37. Liao T, Wang L, Halm ST, Lu L, Fyffe REW, Halm DR. K+ channel KVLQT1 located in the basolateral membrane of distal colonic epithelium is not essential for activating $\mathrm{Cl}$ secretion. Am J Physiol Cell Physiol. 2005;289(3):C564-75. doi:10.1152/ajpcell.00561.2004

38. Linden DR, Sharkey KA, Mawe GM. Enhanced excitability of myenteric AH neurones in the inflamed guinea-pig distal colon. J Physiol. 2003;547(Pt 2):589-601. doi:10.1113/jphysiol.2002.035147

39. Liu C, Lin C, Whitaker DT, et al. Prickle1 is expressed in distinct cell populations of the central nervous system and contributes to neuronal morphogenesis. Hum Mol Genet. 2013;22(11):2234-2246. doi:10.1093/hmg/ddt075

40. Lottig L, Bader S, Jimenez M, Diener M. Evidence for metabotropic function of epithelial nicotinic cholinergic receptors in rat colon. Br J Pharmacol. 2019;176(9):1328-1340. doi:10.1111/bph.14638

41. Lundgren O, Peregrin AT, Persson K, Kordasti S, Uhnoo I, Svensson L. Role of the enteric nervous system in the fluid and electrolyte secretion of rotavirus diarrhea. Science. 2000;287(5452):491-495. doi:10.1126/science.287.5452.491 
42. MacVinish LJ, Hickman ME, Mufti DA, Durrington HJ, Cuthbert AW. Importance of basolateral $\mathrm{K}+$ conductance in maintaining $\mathrm{Cl}$ - secretion in murine nasal and colonic epithelia. J Physiol. 1998;510 ( Pt 1(Pt 1):237-247. doi:10.1111/j.1469-7793.1998.237bz.x

43. Mearin F, Lacy BE, Chang L, et al. Bowel Disorders. Gastroenterology. Published online February 2016. doi:10.1053/j.gastro.2016.02.031

44. Miceli F, Soldovieri MV, Martire M, Taglialatela M. Molecular pharmacology and therapeutic potential of neuronal Kv7-modulating drugs. Curr Opin Pharmacol. 2008;8(1):65-74. doi:10.1016/j.coph.2007.10.003

45. Michel MC, Radziszewski P, Falconer C, Marschall-Kehrel D, Blot K. Unexpected frequent hepatotoxicity of a prescription drug, flupirtine, marketed for about 30 years. $\mathrm{Br}$ J Clin Pharmacol. 2012;73(5):821-825. doi:10.1111/j.1365-2125.2011.04138.x

46. Ng FL, Davis AJ, Jepps TA, et al. Expression and function of the K+ channel KCNQ genes in human arteries. Br J Pharmacol. 2011;162(1):42-53. doi:10.1111/j.14765381.2010.01027.x

47. Page JG, Dirnberger GM. Treatment of the irritable bowel syndrome with Bentyl (dicyclomine hydrochloride). J Clin Gastroenterol. 1981;3(2):153-156. doi:10.1097/00004836-198106000-00009

48. Palmer JM, Wong-Riley M, Sharkey KA. Functional alterations in jejunal myenteric neurons during inflammation in nematode-infected guinea pigs. Am J Physiol. 1998;275(5):G922-35. doi:10.1152/ajpgi.1998.275.5.G922

49. Peiris M, Hockley JR, Reed DE, Smith ESJ, Bulmer DC, Blackshaw LA. Peripheral $\mathrm{K}(\mathrm{V}) 7$ channels regulate visceral sensory function in mouse and human colon. Mol Pain. 2017;13:1744806917709371. doi:10.1177/1744806917709371

50. Peters HC, Hu H, Pongs O, Storm JF, Isbrandt D. Conditional transgenic suppression of $\mathrm{M}$ channels in mouse brain reveals functions in neuronal excitability, resonance and behavior. Nat Neurosci. 2005;8(1):51-60. doi:10.1038/nn1375

51. Roche JP, Westenbroek R, Sorom AJ, Hille B, Mackie K, Shapiro MS. Antibodies and a cysteine-modifying reagent show correspondence of $\mathrm{M}$ current in neurons to KCNQ2 and KCNQ3 K+ channels. Br J Pharmacol. 2002;137(8):1173-1186. doi:10.1038/sj.bjp.0704989

52. Rottgen TS, Nickerson AJ, Minor EA, Stewart AB, Harold AD, Rajendran VM. Dextran sulfate sodium-induced chronic colitis attenuates $\mathrm{Ca}(2+)$-activated $\mathrm{Cl}(-)$ secretion in murine colon by downregulating TMEM16A. Am J Physiol Cell Physiol. 2018;315(1):C10-C20. doi:10.1152/ajpcell.00328.2017

53. Saha L. Irritable bowel syndrome: pathogenesis, diagnosis, treatment, and evidence-based medicine. World J Gastroenterol. 2014;20(22):6759-6773. doi:10.3748/wjg.v20.i22.6759

54. Shah MM, Migliore M, Valencia I, Cooper EC, Brown DA. Functional significance of axonal Kv7 channels in hippocampal pyramidal neurons. Proc Natl Acad Sci U S A. 2008;105(22):7869-7874. doi:10.1073/pnas.0802805105

55. Singal R, Gupta P, Jain N, Gupta S. Role of flupirtine in the treatment of pain - chemistry and its effects. Maedica (Buchar). 2012;7(2):163-166. 
56. Soh H, Pant R, LoTurco JJ, Tzingounis A V. Conditional deletions of epilepsy-associated KCNQ2 and KCNQ3 channels from cerebral cortex cause differential effects on neuronal excitability. $J$ Neurosci. 2014;34(15):5311-5321. doi:10.1523/JNEUROSCI.3919-13.2014

57. Stutts MJ, Lazarowski ER, Paradiso AM, Boucher RC. Activation of CFTR Clconductance in polarized T84 cells by luminal extracellular ATP. Am J Physiol. 1995;268(2 Pt 1):C425-33. doi:10.1152/ajpcell.1995.268.2.C425

58. Sun H, Harris WT, Kortyka S, et al. Tgf-beta downregulation of distinct chloride channels in cystic fibrosis-affected epithelia. PLoS One. 2014;9(9):e106842. doi:10.1371/journal.pone.0106842

59. Wang HS, Pan Z, Shi W, et al. KCNQ2 and KCNQ3 potassium channel subunits: molecular correlates of the M-channel. Science. 1998;282(5395):1890-1893. doi:10.1126/science.282.5395.1890

60. Watanabe H, Nagata E, Kosakai A, et al. Disruption of the epilepsy KCNQ2 gene results in neural hyperexcitability. J Neurochem. 2000;75(1):28-33. doi:10.1046/j.14714159.2000.0750028.x

61. Zhou J-J, Gao Y, Kosten TA, Zhao Z, Li D-P. Acute stress diminishes M-current contributing to elevated activity of hypothalamic-pituitary-adrenal axis. Neuropharmacology. 2017;114:67-76. doi:10.1016/j.neuropharm.2016.11.024 


\section{FIGURES AND LEGENDS}

A

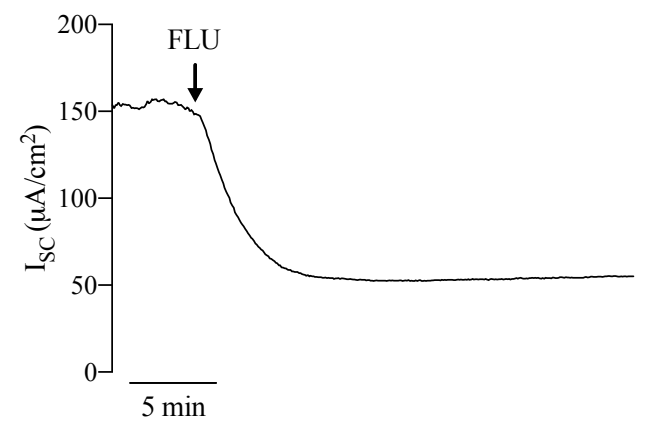

C

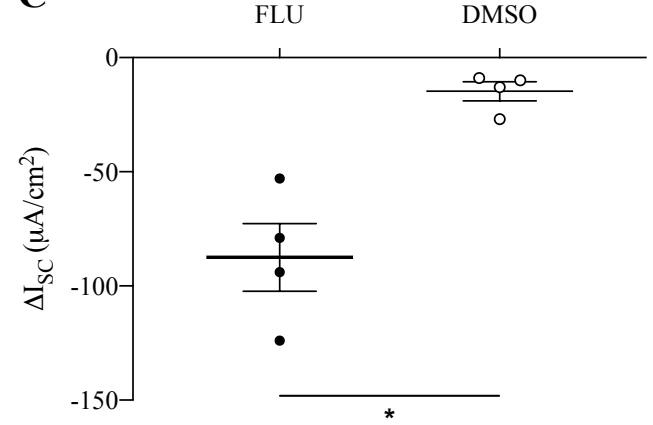

B

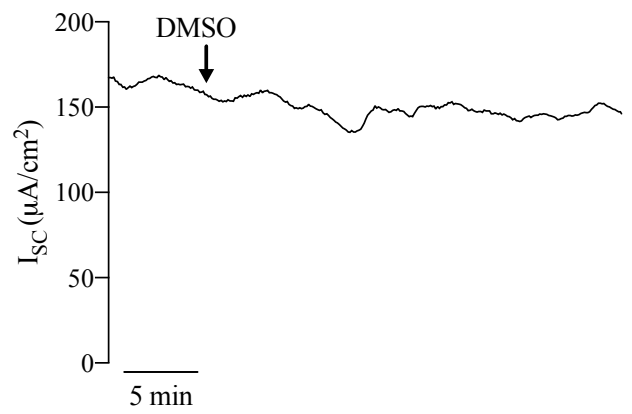

D

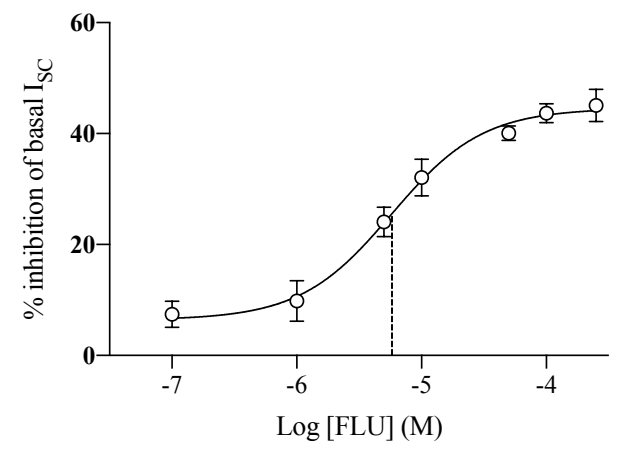

Figure 1. Flupirtine inhibits basal $I_{S C}$ in mouse distal colon in a concentration-dependent manner in mouse distal colon. Representative $\mathrm{I}_{\mathrm{SC}}$ recording of unstripped mouse distal colon treated with $100 \mu \mathrm{M}$ flupirtine (FLU) $(A)$ or vehicle (DMSO) $(B)$ in the serosal bath. $C$ : Group data showing the change in $\mathrm{I}_{\mathrm{SC}}\left(\Delta \mathrm{I}_{\mathrm{SC}}\right)$ in response to FLU or DMSO treatment. $D$ : FLU concentration-response curve fitted using non-linear regression. Half-maximal inhibitory concentration $\left(\mathrm{IC}_{50}\right)$ for FLU was calculated to be $5.8 \mu \mathrm{M}$. As mentioned above, correction was applied for time and for vehicle administration for subsequent concentrations of flupirtine, based upon $\mathrm{I}_{\mathrm{SC}}$ recordings from adjacent tissues $(n=4)$ treated only with DMSO at each corresponding time/concentration point. Those values, subtracted from the $\mathrm{DI}_{\mathrm{SC}(\mathrm{FLU})}$ from $0.1,1,5,10,50,100$ 
and $250 \mu \mathrm{M}$ flupirtine concentrations, were $5.0,16.3,22.3,31.5,45.3,47.5$ and $50.5 \mu \mathrm{A} / \mathrm{cm}^{2}$, respectively. These adjusted values were used for fitting of the concentration/response curve. Additionally, the "top" and "bottom" parameters of the non-linear fit were calculated to be $44.6 \pm$ 2.3 and $6.4 \pm 3.1 \mu \mathrm{A} / \mathrm{cm}^{2}$, respectively. The "bottom" parameter value $(6.4 \pm 3.1 \mu \mathrm{A} / \mathrm{cm} 2)$ was not significantly different from $0.0(p=0.11)$. The "Hill slope" parameter was calculated as $1.18 \pm$ 0.4 , and this was not significantly different from $1.0(p=0.60)$. Data are presented as mean \pm SEM from 4 separate animals. ${ }^{*} p<0.05$.

A

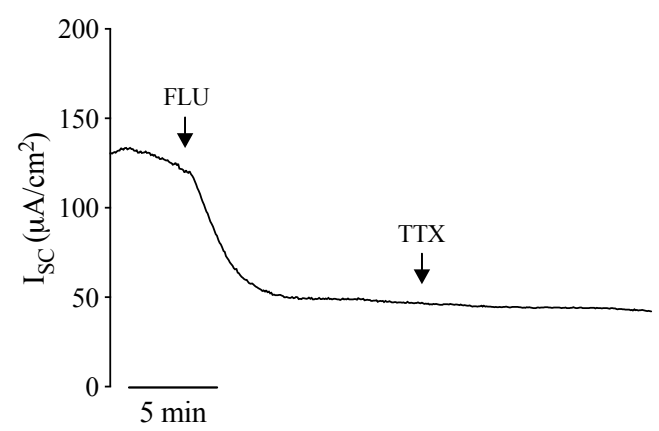

C

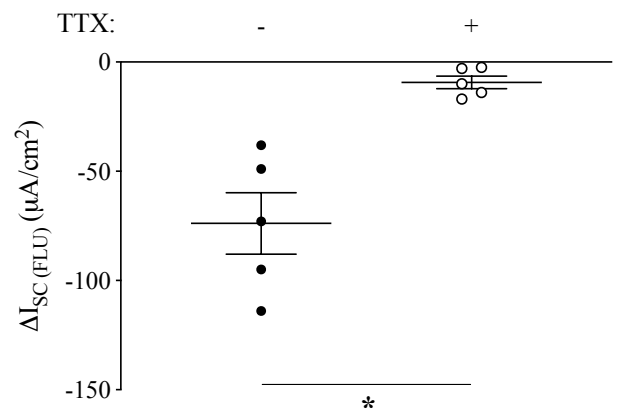

B

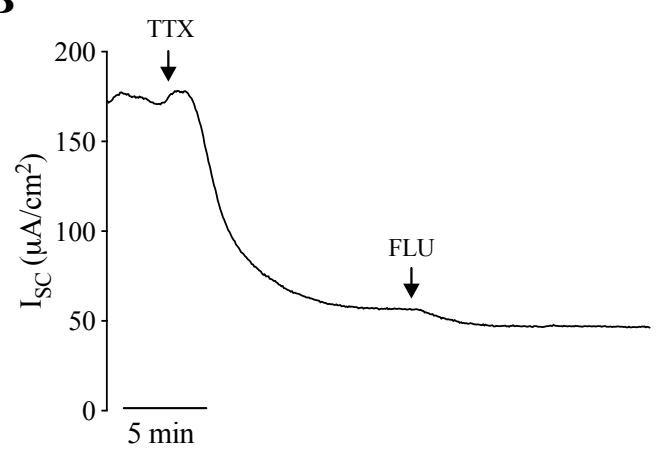

D

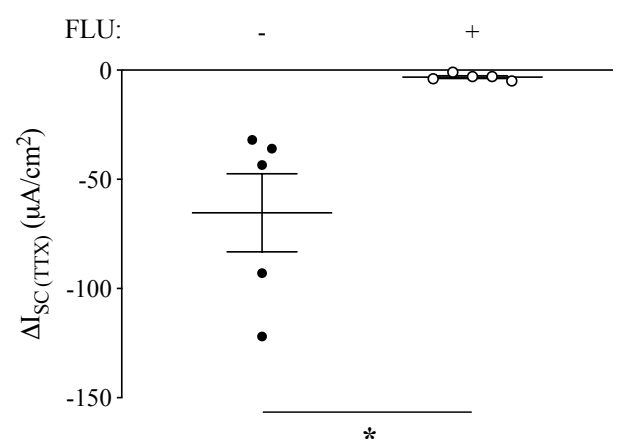

Figure 2. Flupirtine and tetrodotoxin inhibit basal $I_{S C}$ in a similar and non-additive manner in mouse distal colon. $A$ : Representative $\mathrm{I}_{\mathrm{SC}}$ recording of unstripped mouse distal colon treated with $100 \mu \mathrm{M}$ flupirtine (FLU), followed by $1 \mu \mathrm{M}$ tetrodotoxin (TTX) in the serosal bath. $B$ : 
Representative $\mathrm{I}_{\mathrm{SC}}$ recording of unstripped mouse distal colon treated with $1 \mu \mathrm{M}$ TTX, followed by $100 \mu \mathrm{M}$ FLU in the serosal bath. $C$ : Comparison of the change in ISC induced by $100 \mu \mathrm{M}$ FLU $\left(\Delta \mathrm{I}_{\mathrm{SC}(\mathrm{FLU})}\right)$ in the presence and absence of $1 \mu \mathrm{M}$ TTX. $D$ : Comparison of the change in $\mathrm{I}_{\mathrm{SC}}$ induced by $1 \mu \mathrm{M}$ TTX $\left(\Delta \mathrm{I}_{\mathrm{SC}(\mathrm{TTX})}\right)$ in the presence and absence of $100 \mu \mathrm{M}$ FLU. Data are presented as mean \pm SEM from 5 different animals. ${ }^{*} p<0.05$.

A

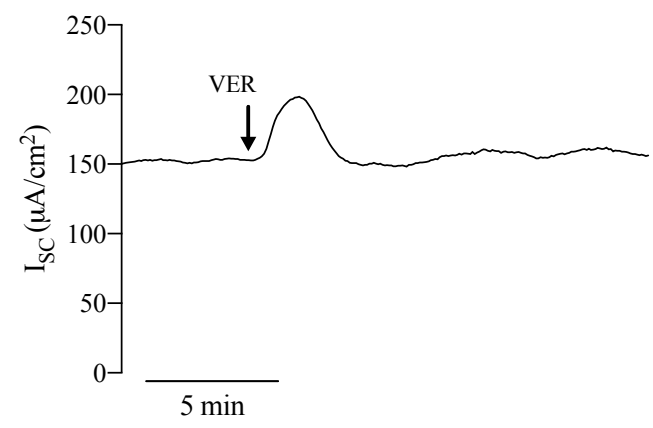

C

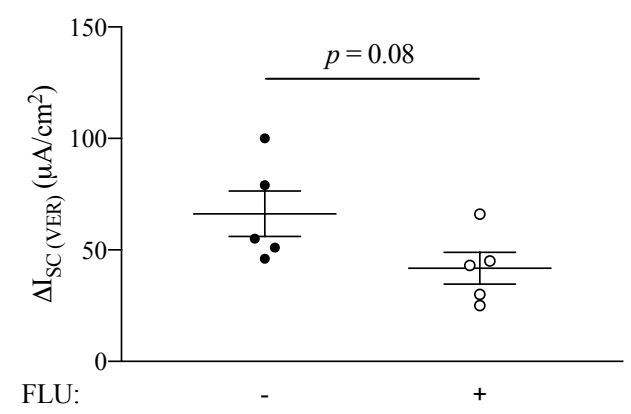

B

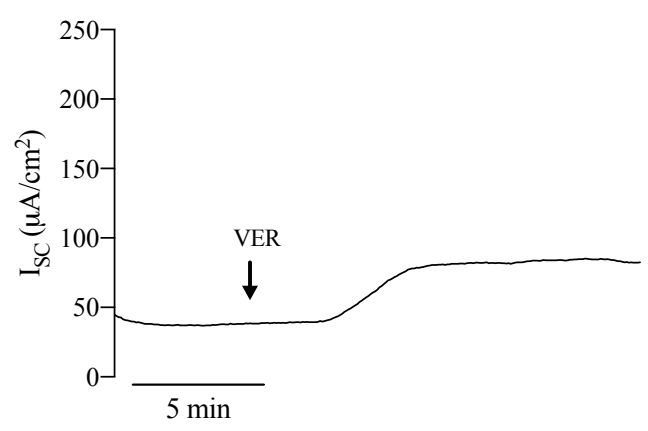

D

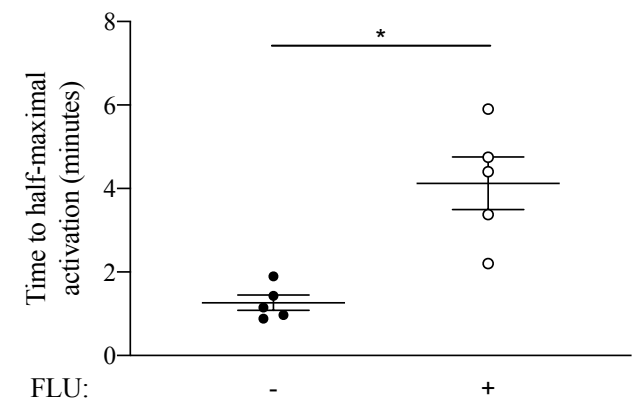

Figure 3. Flupirtine delays the response to veratridine in mouse distal colon. $A$ : Representative $\mathrm{I}_{\mathrm{SC}}$ recording of unstripped mouse distal colon treated with $10 \mu \mathrm{M}$ veratridine (VER) in the serosal bath. $B$ : Representative $\mathrm{I}_{\mathrm{SC}}$ recording of unstripped mouse distal colon treated with $10 \mu \mathrm{M}$ VER in the presence of $100 \mu \mathrm{M}$ flupirtine (FLU). C: Comparison of the change in $\mathrm{I}_{\mathrm{SC}}$ induced by 10 $\mu \mathrm{M} \operatorname{VER}\left(\Delta \mathrm{I}_{\mathrm{SC}(\mathrm{VER})}\right)$ in the presence $(+)$ and absence (-) of $100 \mu \mathrm{M}$ FLU. D: Comparison of the time to half-maximal activation following VER administration in the presence $(+)$ and absence 
(-) of $100 \mu \mathrm{M}$ FLU. Data are presented as mean \pm SEM from 5 different animals. ${ }^{*} p<0.05$.

A

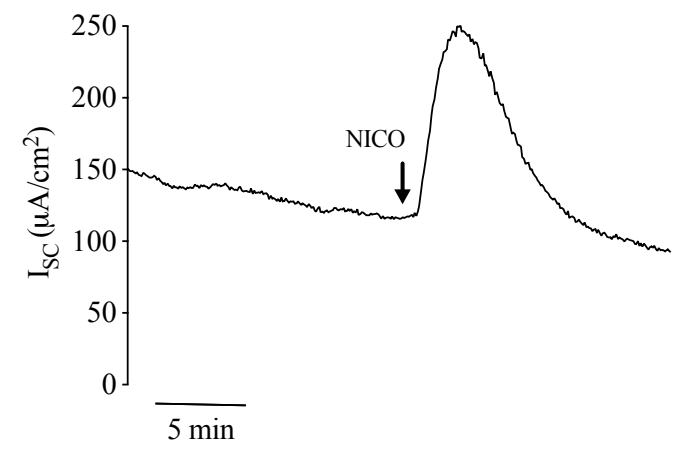

B

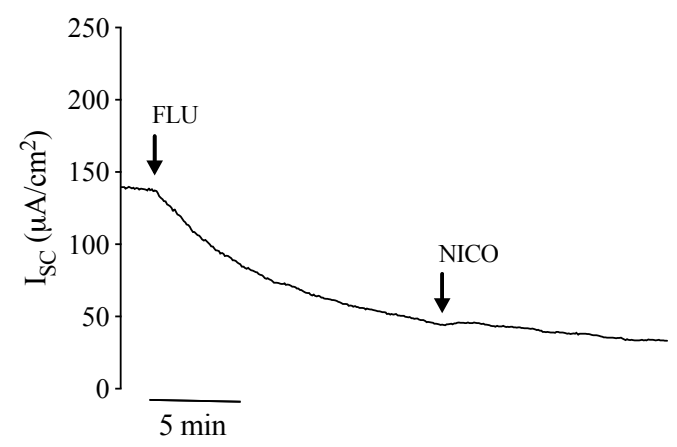

C

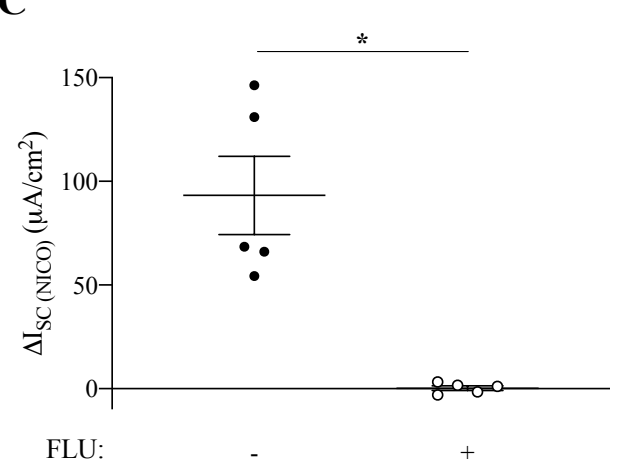

Figure 4. Flupirtine abolishes the response to nicotine in mouse distal colon. $A$ : Representative $\mathrm{I}_{\mathrm{SC}}$ recordings of unstripped mouse distal colon treated with $100 \mu \mathrm{M}$ nicotine (NICO) in the serosal bath. $B$ : Representative $\mathrm{I}_{\mathrm{SC}}$ recording of unstripped mouse distal colon treated with $100 \mu \mathrm{M}$ NICO in the presence of $100 \mu \mathrm{M}$ flupirtine (FLU). C: Comparison of the change in ISC induced by 100 $\mu \mathrm{M}$ NICO $\left(\Delta \mathrm{I}_{\mathrm{SC}(\mathrm{NICO})}\right)$ in the presence $(+)$ and absence $(-)$ of $100 \mu \mathrm{M}$ FLU. Data are presented as mean \pm SEM from 5 different animals. ${ }^{*} p<0.05$. 
$\mathbf{A}$

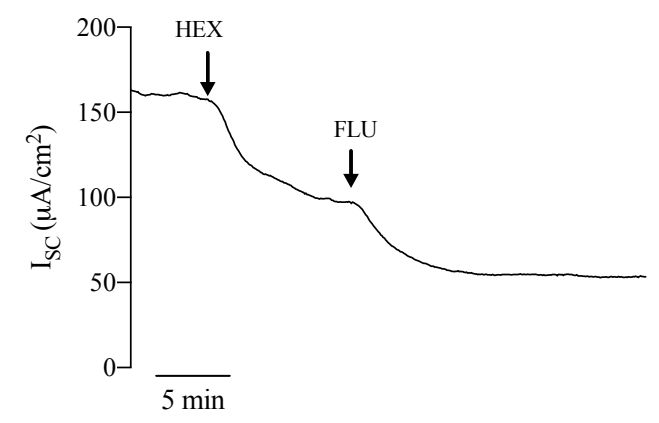

C

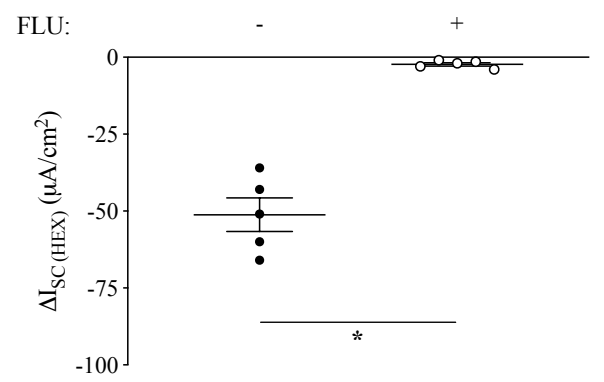

B

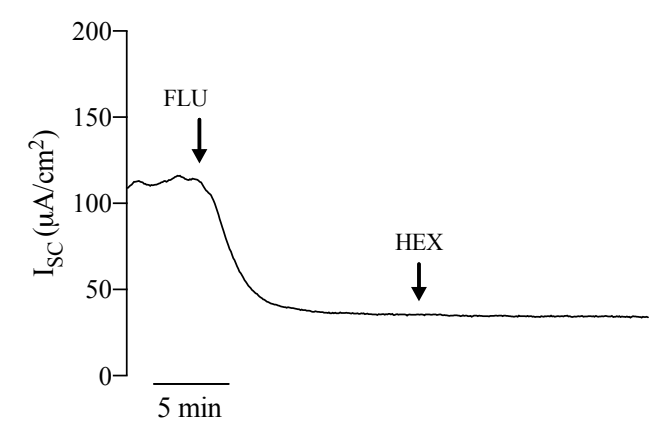

D

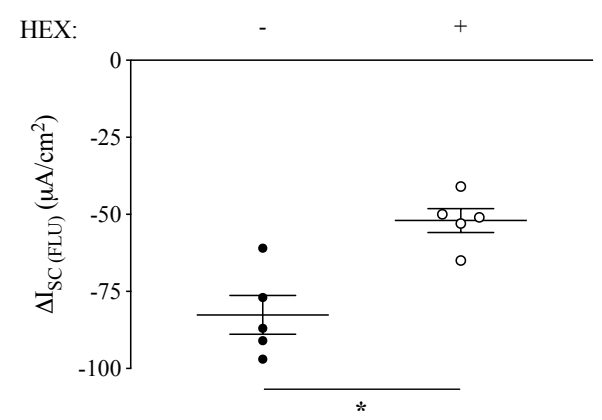

Figure 5. Flupirtine abolishes the response to hexamethonium in mouse distal colon. $A$ :

Representative $\mathrm{I}_{\mathrm{SC}}$ recordings of unstripped mouse distal colon treated with $100 \mu \mathrm{M}$ hexamethonium (HEX), followed by $100 \mu \mathrm{M}$ flupirtine (FLU) in the serosal bath. $B$ : Representative $\mathrm{I}_{\mathrm{SC}}$ recordings of unstripped mouse distal colon treated with $100 \mu \mathrm{M}$ HEX in the presence of $100 \mu \mathrm{M}$ FLU. $C$ : Comparison of the change in $\mathrm{I}_{\mathrm{SC}}$ induced by $100 \mu \mathrm{M} \operatorname{HEX}\left(\Delta \mathrm{I}_{\mathrm{SC}(\mathrm{HEX})}\right)$ in the presence $(+)$ and absence (-) of $100 \mu \mathrm{M}$ FLU. G: Comparison of the change in ISC induced by $100 \mu \mathrm{M}$ FLU $\left(\Delta \mathrm{I}_{\mathrm{SC}(\mathrm{FLU})}\right)$ in the presence $(+)$ and absence $(-)$ of $100 \mu \mathrm{M}$ HEX. Data are presented as mean \pm SEM from 5 different animals. ${ }^{*} p<0.05$. 
A

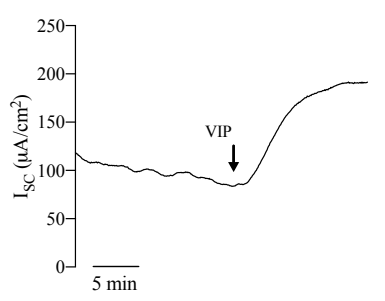

D

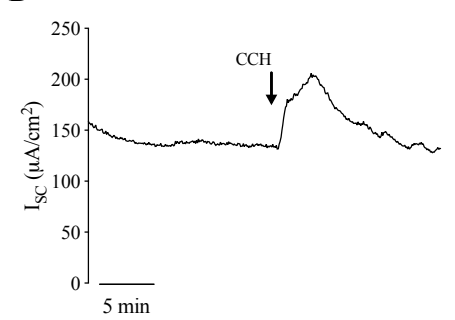

B

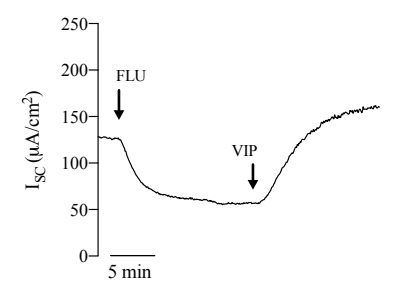

$\mathbf{E}$

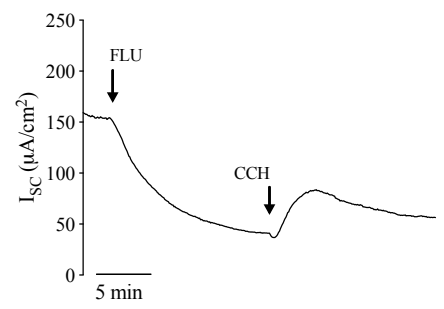

C

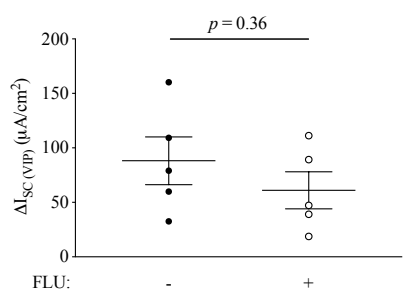

$\mathbf{F}$

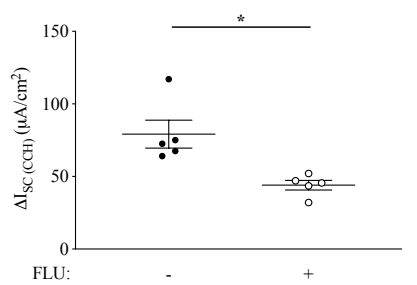

Figure 6. Flupirtine does not abolish the response to VIP or carbachol in mouse distal colon.

A: Representative $\mathrm{I}_{\mathrm{SC}}$ recordings of unstripped mouse distal colon treated with $100 \mathrm{nM}$ vasoactive intestinal polypeptide (VIP) in the serosal bath. $B$ : Representative ISC $_{\mathrm{SC}}$ recordings of full-thickness mouse distal colon pre-treated with $100 \mu \mathrm{M}$ flupirtine (FLU), followed by subsequent addition of $100 \mathrm{nM}$ VIP to the serosal bath. $C$ : Comparison of the change in $\mathrm{I}_{\mathrm{SC}}$ induced by $100 \mathrm{nM}$ VIP $\left(\Delta \mathrm{I}_{\mathrm{SC}(\mathrm{VIP})}\right)$ in the presence (+) and absence (-) of $100 \mu \mathrm{M}$ FLU. D: Representative $\mathrm{I}_{\mathrm{SC}}$ recordings of unstripped mouse distal colon treated with $100 \mu \mathrm{M}$ carbachol $(\mathrm{CCH})$ in the serosal bath. $E$ : Representative $\mathrm{I}_{\mathrm{SC}}$ recordings of unstripped mouse distal colon pre-treated with $100 \mu \mathrm{M}$ FLU, followed by subsequent addition of $100 \mu \mathrm{M} \mathrm{CCH}$ to the serosal bath. $F$ : Comparison of the change in $\mathrm{I}_{\mathrm{SC}}$ induced by $100 \mu \mathrm{M} \mathrm{CCH}\left(\Delta \mathrm{I}_{\mathrm{SC}(\mathrm{CCH})}\right)$ in the presence (+) and absence (-) of $100 \mu \mathrm{M}$ FLU. Data are presented as mean \pm SEM from 5 different animals. ${ }^{*} p<0.05$. 
A

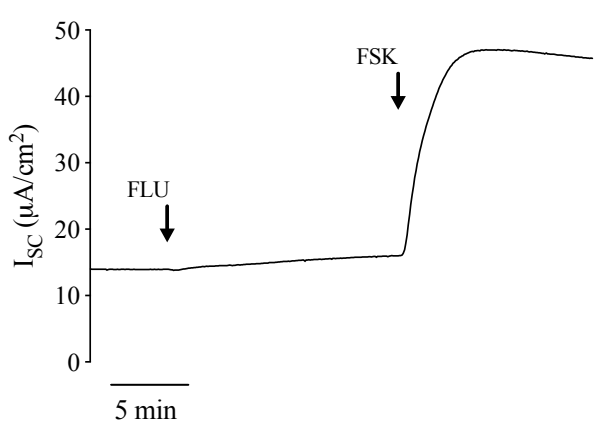

B

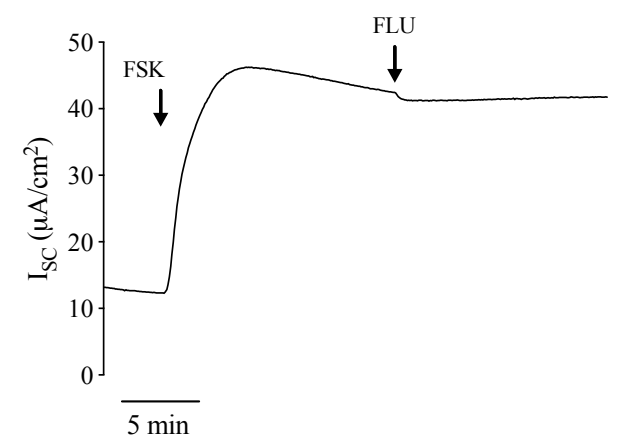

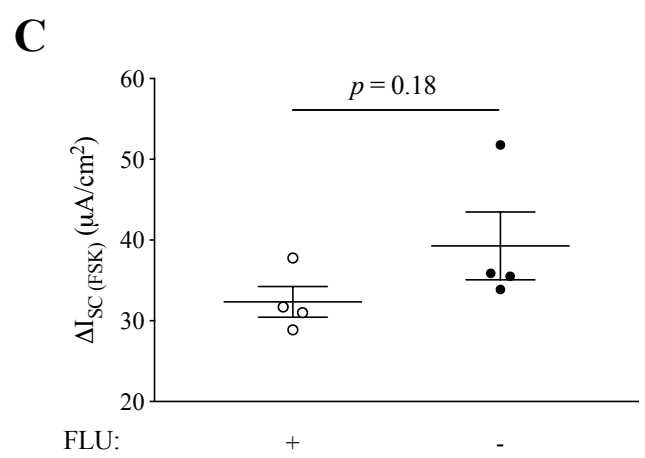

Figure 7. Flupirtine does not inhibit $\mathrm{Cl}^{-}$secretion in $\mathbf{T} 84$ Monolayers. $A$ : Representative $\mathrm{I}_{\mathrm{SC}}$ recording from T84 monolayers that were treated with basolateral $100 \mu \mathrm{M}$ flupirtine (FLU), followed by $10 \mu \mathrm{M}$ forskolin (FSK). B: Representative $\mathrm{I}_{\mathrm{SC}}$ recording from T84 monolayers that were treated with $10 \mu \mathrm{M}$ FSK, followed by $100 \mu \mathrm{M}$ FLU. $C$ : Comparison of the change in $\mathrm{I}_{\mathrm{SC}}$ induced by $10 \mu \mathrm{M}$ FSK $\left(\Delta \mathrm{I}_{\mathrm{SC}(\mathrm{FSK})}\right)$ in the presence $(+)$ and absence (-) of $100 \mu \mathrm{M}$ FLU. Data are presented as mean \pm SEM from 4 different experiments. ${ }^{*} p<0.05$. 

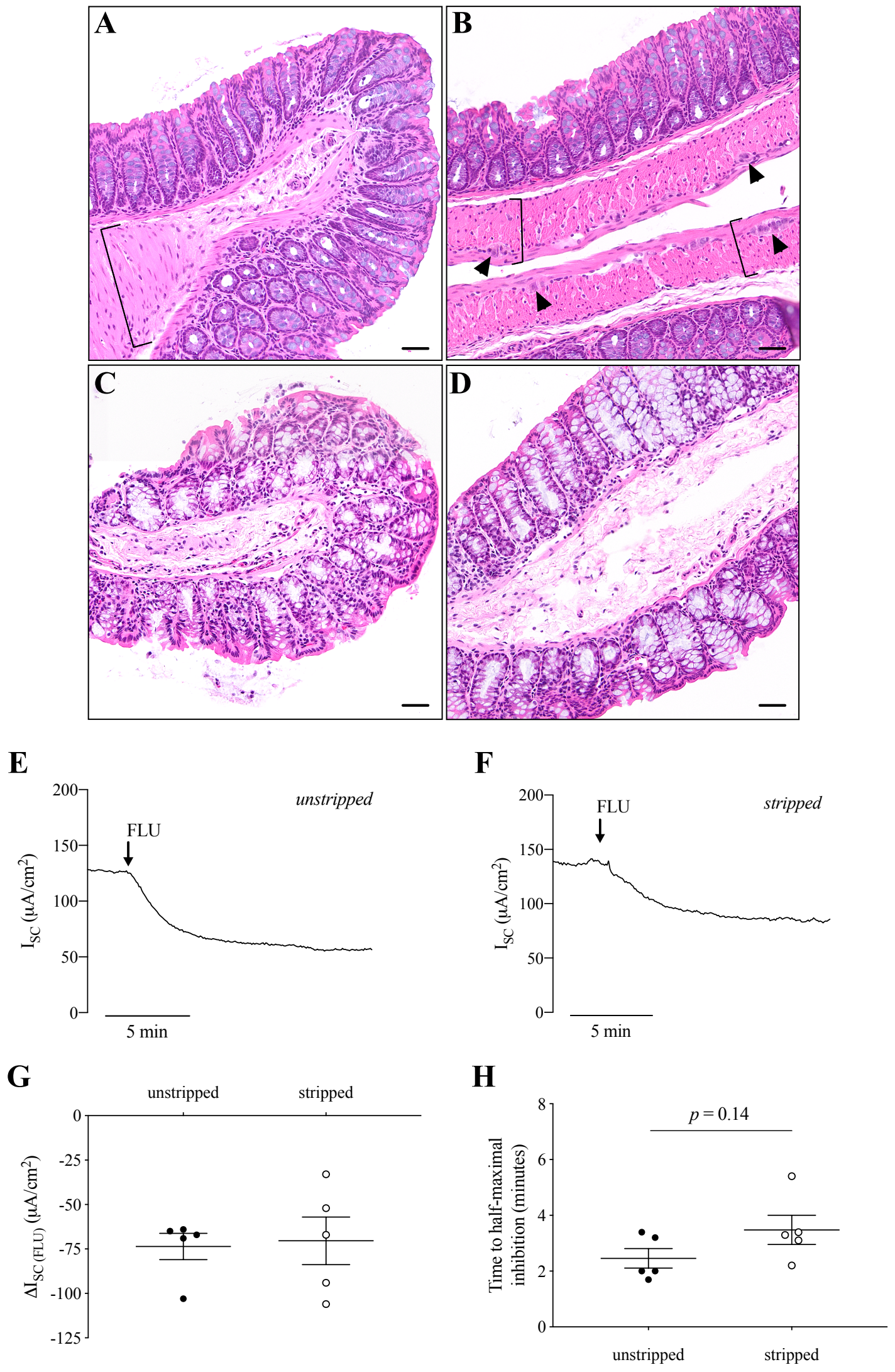
Figure 8. Flupirtine inhibits basal $\mathrm{Cl}^{-}$secretion in both full-thickness and seromuscularstripped mouse distal colon. Hematoxylin and eosin stained mouse distal colonic tissue in unstripped $(A-B)$ and seromuscular-stripped $(C-D)$ preparations. Brackets in panels A and B mark the muscularis propria in unstripped tissues which is absent in seromuscular-stripped preparations. Brackets in panel B show the outer longitudinal and inner circular smooth muscle layers, with arrowheads indicating the presence of myenteric ganglia between these two layers. Scale bars $=$ $50 \mu \mathrm{m}$. Representative $\mathrm{I}_{\mathrm{SC}}$ recording of unstripped $(E)$ and seromuscular-stripped $(F)$ mouse distal colon treated with $100 \mu \mathrm{M}$ flupirtine (FLU) in the serosal bath. G: Comparison of the change in $\mathrm{I}_{\mathrm{SC}}$ induced by $100 \mu \mathrm{M}$ FLU $\left(\Delta \mathrm{I}_{\mathrm{SC}(\mathrm{FLU})}\right)$ in unstripped and seromuscular-stripped mouse distal colon. $H$ : Comparison of the time necessary to reach half-maximal inhibition following basolateral addition of $100 \mu \mathrm{M}$ FLU in unstripped and seromuscular-stripped mouse distal colon. Data are presented as mean \pm SEM from 5 different animals. $* p<0.05$ 
A

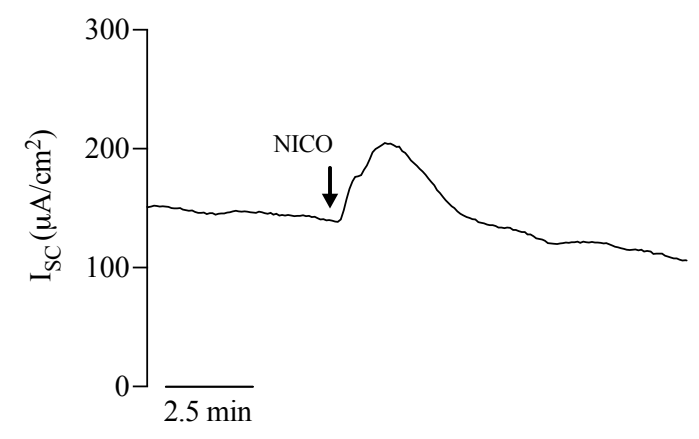

C

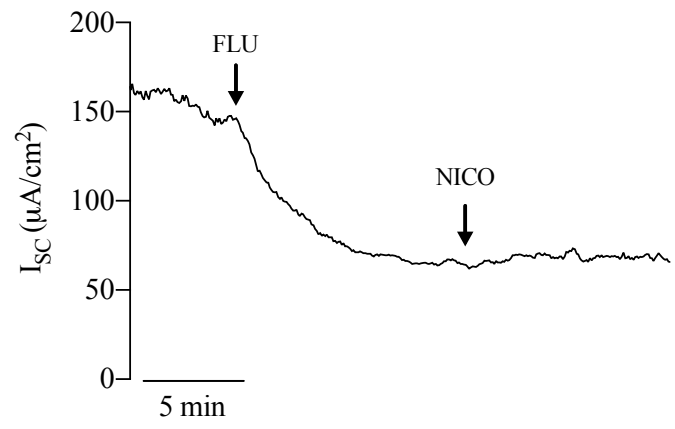

$\mathbf{E}$

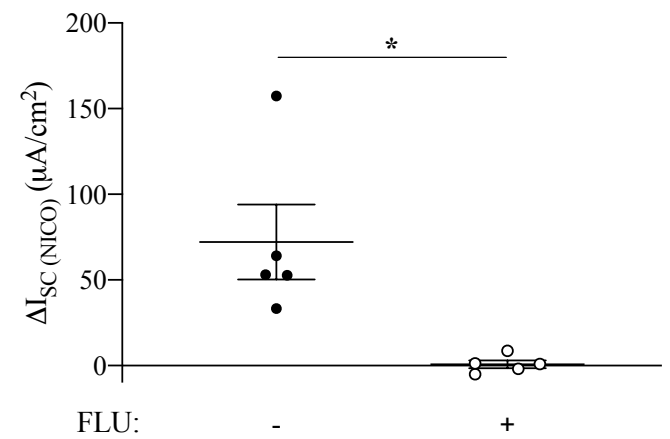

B

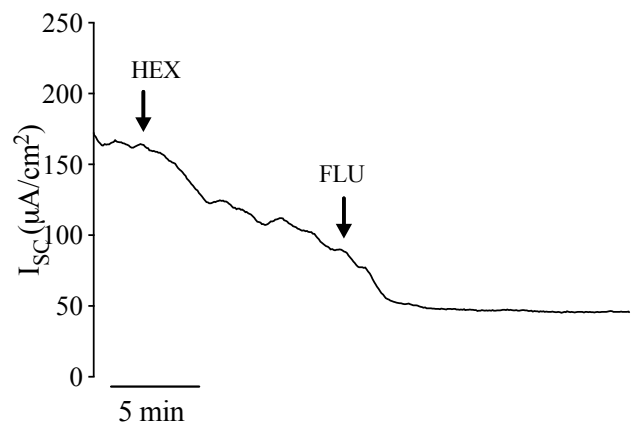

D

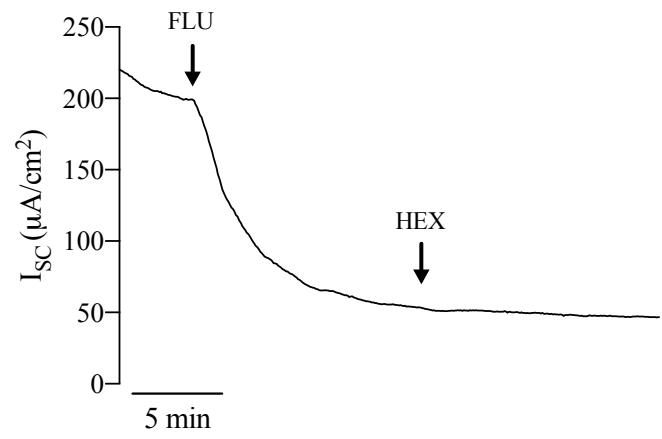

$\mathbf{F}$

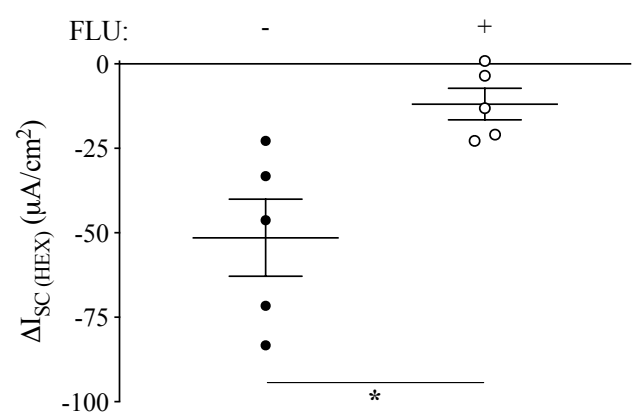

Figure 9. Flupirtine inhibits the response to nicotine and hexamethonium in seromuscularstripped mouse distal colon. Representative $\mathrm{I}_{\mathrm{SC}}$ recording of stripped mouse distal colon treated with $100 \mu \mathrm{M}$ nicotine (NICO) (A) and $200 \mu \mathrm{M}$ hexamethonium (HEX) (B) in the serosal bath. Representative $\mathrm{I}_{\mathrm{SC}}$ recordings of stripped mouse distal colon pre-treated with $100 \mu \mathrm{M}$ flupirtine (FLU), followed by subsequent addition of $100 \mu \mathrm{M}$ NICO (C) or $200 \mu \mathrm{M}$ HEX (D) to the serosal 
bath. Comparison of the change in ISC induced by $100 \mu \mathrm{M}$ NICO or $200 \mu \mathrm{M}$ HEX in the presence $(+)$ and absence (-) of $100 \mu \mathrm{M}$ FLU. Data are presented as mean \pm SEM from 5 different animals. ${ }^{*} p<0.05$.

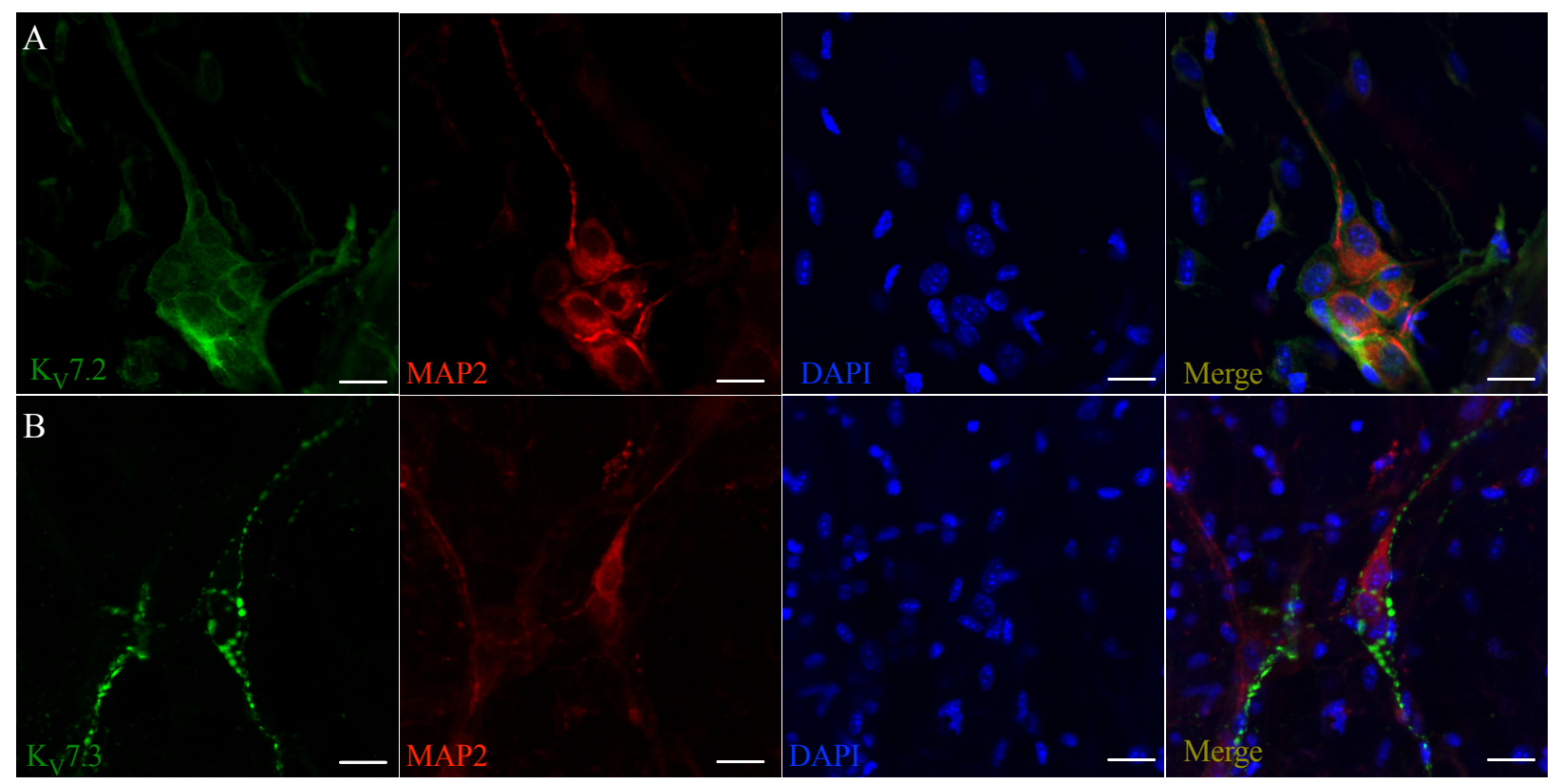

Figure 10. $K_{v} 7.2$ and $K_{v} 7.3$ channel subtypes are expressed in the submucosal plexus of mouse distal colon. Immunofluorescent labelling of whole-mount preparation of mouse distal colon submucosa, captured at 20x magnification. Row A: Whole-mount submucosa labelled for $\mathrm{K}_{\mathrm{V}} 7.2$ (green), microtubule-associated protein 2 (MAP2; red), and nuclei (DAPI; blue), as well as a merge of the red, green and blue channels. Row B: Whole-mount submucosa labelled for $\mathrm{K}_{\mathrm{V}} 7.3$ (green), microtubule-associated protein 2 (MAP2; red), and nuclei (DAPI; blue), as well as a merge of the red, green and blue channels. Scale bars $=20 \mu \mathrm{m}$. 
A

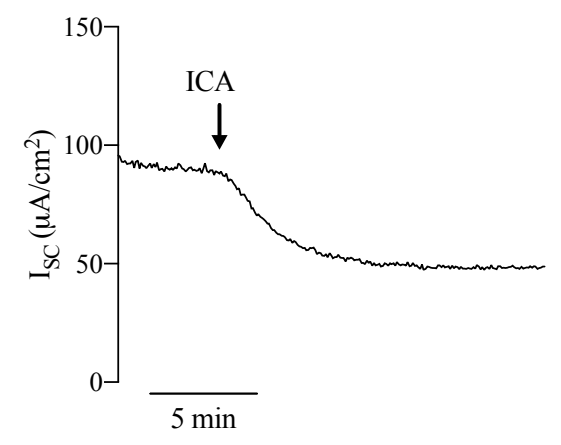

B

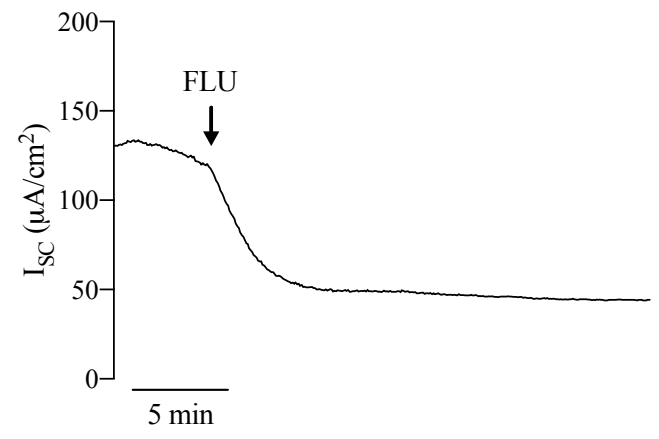

C

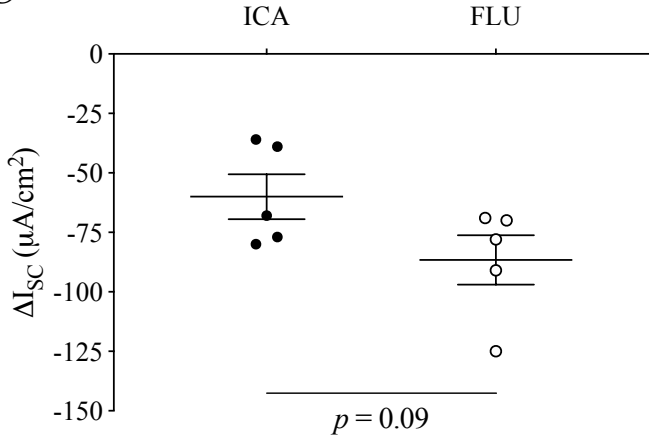

Figure 11. ICA 110381, a specific activator of $\mathrm{K}_{\mathrm{V}} 7.2 / 3$, inhibits basal $\mathrm{Cl}^{-}$secretion in mouse distal colon. $A$ : Representative $\mathrm{I}_{\mathrm{SC}}$ recording of unstripped mouse distal colon treated with 100 $\mu \mathrm{M}$ ICA 110381 (ICA) in the serosal bath. $B$ : Representative $\mathrm{I}_{\mathrm{SC}}$ recording of unstripped mouse distal colon treated with $100 \mu \mathrm{M}$ flupirtine (FLU) in the serosal bath. $C$ : Comparison of the change in $\mathrm{I}_{\mathrm{SC}}\left(\Delta \mathrm{I}_{\mathrm{SC}}\right)$ induced by $100 \mu \mathrm{M}$ ICA and $100 \mu \mathrm{M}$ flupirtine (FLU). Data are presented as mean \pm SEM from 5 different animals 


\section{CHAPTER 3}

TITLE: Flupirtine enhances NHE-3 mediated $\mathrm{Na}^{+}$absorption in rat colon via an ENS-dependent mechanism

RUNNING TITLE: Flupirtine enhances colonic $\mathrm{Na}^{+}$absorption

AUTHORS: Andrew J. Nickerson ${ }^{1,2}$ and Vazhaikkurichi M. Rajendran ${ }^{2,3, *}$

1Departments of Physiology, Pharmacology and Neuroscience, West Virginia University School of Medicine, Morgantown, West Virginia

2Department of Biochemistry, West Virginia University School of Medicine, Morgantown, West Virginia

3Department of Medicine, West Virginia University School of Medicine, Morgantown, West Virginia

VMR ORCID: 0000-0002-8999-8721

AJN and VMR conceived of and designed the experiments. AJN performed all experiments and wrote the manuscript. AJN and VMR edited and prepared the manuscript in its current version.

* address all correspondence

Vazhaikkurichi M. Rajendran, $\mathrm{PhD}$.

Departments of Biochemistry and Medicine

West Virginia University School of Medicine

1 Medical Center Drive

Morgantown, WV 26506

Phone: (304) 293-0510

Email: vrajendran@hsc.wvu.edu 


\section{ABSTRACT}

Recent studies in our lab have shown that the $\mathrm{K}_{\mathrm{V}} 7$ channel activator, flupirtine, inhibits colonic epithelial $\mathrm{Cl}^{-}$secretion through effects on submucosal neurons of the enteric nervous system (ENS). We hypothesized that flupirtine would also stimulate $\mathrm{Na}^{+}$absorption as a result of reduced secretory ENS input to the epithelium. To test this hypothesis, unidirectional ${ }^{22} \mathrm{Na}^{+}$fluxes were measured under voltage-clamped conditions. Pharmacological approaches using an Ussingstyle recording chamber, combined immunofluorescence microscopy techniques were used to determine the effect of flupirtine on active $\mathrm{Na}^{+}$transport in the rat colon. Flupirtine stimulated electroneutral $\mathrm{Na}^{+}$absorption in partially seromuscular stripped colonic tissues, while simultaneously inhibiting short circuit current ( $\mathrm{I}_{\mathrm{SC}}$; i.e., $\mathrm{Cl}^{-}$secretion). Both of these effects were attenuated by pre-treatment with the ENS inhibitor, tetrodotoxin. The NHE-3-selective inhibitor, S3226, significantly inhibited flupirtine-stimulated $\mathrm{Na}^{+}$absorption whereas the NHE-2-selective inhibitor HOE-694 did not. NHE-3 localization near the apical membranes of surface epithelial cells was also more apparent in flupirtine-treated colon versus control. Flupirtine did not alter epithelial $\mathrm{Na}^{+}$channel (ENaC)-mediated $\mathrm{Na}^{+}$absorption in distal colonic tissues obtained from hyperaldosteronaemic rats and had no effect in the normal ileum, but did stimulate $\mathrm{Na}^{+}$absorption in the proximal colon. Finally, the parallel effects of flupirtine on $\mathrm{ISC}_{\mathrm{SC}}\left(\mathrm{Cl}^{-}\right.$secretion $)$and $\mathrm{Na}^{+}$ absorption were significantly correlated with each other. Together, these data indicate that flupirtine stimulates NHE-3-dependent $\mathrm{Na}^{+}$absorption, likely as a result of reduced stimulatory input to the colonic epithelium by submucosal ENS neurons. 
NEW AND NOTEWORTHY: We present a novel mechanism regarding regulation of epithelial ion transport by enteric neurons. Activation of neuronal $\mathrm{K}_{\mathrm{V}} 7 \mathrm{~K}^{+}$channels markedly stimulates $\mathrm{Na}^{+}$ absorption and inhibits $\mathrm{Cl}^{-}$secretion across the colonic epithelium. This may be useful in developing new treatments for diarrheal disorders, such as irritable bowel syndrome with diarrhea (IBS-D).

KEY WORDS: enteric nervous system; Ussing chamber; IBS-D; KCNQ. 


\section{INTRODUCTION}

The epithelium of the colon plays a critical role in maintaining overall electrolyte and fluid homeostasis $(1,2,3,4)$. Although the total volume of absorbed water is typically less than in the small intestine, the colon possesses a hugely dynamic range in absorptive capacity and readily adapts its electrolyte transport activity to fit the needs of the organism (5). Having tremendous influence over this activity is the enteric nervous system (ENS), one of the most important and powerful regulators of epithelial ion transport $(6,7,8,9) . \mathrm{Na}^{+}, \mathrm{Cl}^{-}$and water are actively absorbed under basal conditions, but the release of pro-secretory neurotransmitters from ENS neurons can induce a robust efflux of $\mathrm{Cl}^{-}$and $\mathrm{HCO}_{3}{ }^{-}$from the crypt cells $(10,11)$, driving water into the gut lumen through osmosis. At the same time, ENS-driven secretion is augmented by the concurrent inhibition of basal absorptive processes, such as $\mathrm{Na}^{+} / \mathrm{H}^{+}$and $\mathrm{Cl}^{-} / \mathrm{HCO}_{3}{ }^{-}$exchange in surface epithelial cells $(12,13)$.

Recent work in our lab has revealed that pharmacological activation of voltage-gated $\mathrm{K}_{\mathrm{V}} 7$ (KCNQ) $\mathrm{K}^{+}$channels in the ENS inhibits neurogenic $\mathrm{Cl}^{-}$secretion significantly within the colon (14). This is likely a result of suppressed activity of secretomotor neurons, as $\mathrm{K}_{\mathrm{V}} 7$ channels mediate a hyperpolarizing $\mathrm{K}^{+}$current that reduces excitability and actional potential firing $(15,16,17)$. The importance of these channels has been highlighted within many central and peripheral circuits $(18,19,20)$, and $\mathrm{K}_{\mathrm{V}} 7$ channel activators have been used clinically to treat chronic pain and epilepsy - both of which are related to neuronal hyper-excitability $(16,21,22)$. However, the role of $\mathrm{K}_{\mathrm{V}} 7$ channels in the ENS with respect to epithelial transport remains incompletely understood.

Many common gastrointestinal (GI) disorders, such as irritable bowel syndrome with diarrhea (IBS-D), are related a hyperexcitable ENS $(6,11,23,24)$. IBS-D, while not usually life- 
threating, presents a massive economic burden to the healthcare systems in the U.S. and abroad (25). Many current treatment options that target ENS activity are effective in relieving symptoms but are commonly associated with adverse side effects $(26,27,28)$. Thus, development of improved therapeutic strategies for IBS-D management is of great clinical importance. Gaining a deeper understanding of epithelial transport modulation by $\mathrm{K}_{\mathrm{V}} 7$ channels in the ENS may provide new avenues for treatment, as the use of $\mathrm{K}_{\mathrm{V}} 7$ activators has not yet been explored in this context.

In light of the recent evidence concerning the effects on neurogenic $\mathrm{Cl}^{-}$secretion (14), we predicted that activation of $\mathrm{K}_{\mathrm{V}} 7$ channels in the ENS would also enhance $\mathrm{Na}^{+}$absorption simultaneously, as a result of diminished stimulatory ENS input. $\mathrm{Na}^{+}$absorption - in particular, the activity of the predominant electroneutral $\mathrm{Na}^{+} / \mathrm{H}^{+}$exchanger isoform $3(\mathrm{NHE}-3)-$ is known to be active at rest and inhibited by various ENS-derived neurotransmitters (29). We therefore tested the hypothesis that the $\mathrm{K}_{\mathrm{V}} 7$ channel activator, flupirtine, stimulates active $\mathrm{Na}^{+}$absorption in the colon. Unidirectional ${ }^{22} \mathrm{Na}^{+}$fluxes and short-circuit current $\left(\mathrm{I}_{\mathrm{SC}}\right)$ were measured under voltageclamped conditions in an Ussing chamber system. Our findings suggest that active $\mathrm{Cl}^{-}$secretion is inhibited, while NHE-3-mediated $\mathrm{Na}^{+}$absorption is enhanced by activation of $\mathrm{K}_{\mathrm{V}} 7$ channels within the ENS.

\section{METHODS}

Animals

Non-fasting male Sprague-Dawley rats $(150-200 \mathrm{~g})$ were housed 2 per cage in climatecontrolled rooms under 12-hour light/dark cycles and given free access to standard chow and water. One group of rats was given a substitute $\mathrm{Na}^{+}$-depleted diet for 7 consecutive days to induce secondary hyperaldosteronism, as has been described previously (30,31). Rats given $\mathrm{Na}^{+}$-depleted 
diet were also allowed free access to water. Dietary $\mathrm{Na}^{+}$depletion was used to study the effect of ENS $\mathrm{K}_{\mathrm{V}} 7$ activation specifically on electrogenic versus electroneutral epithelial $\mathrm{Na}^{+}$absorption, as electroneutral (primarily NHE-3-mediated) absorption is down-regulated while electrogenic (epithelial $\mathrm{Na}^{+}$channel; ENaC-mediated) absorption is induced in hyperaldosteronaemic rats. ${ }^{32} \mathrm{On}$ the day of experiments, all rats were anesthetized with 5\% isoflurane balanced with oxygen and maintained under a deep plane of anesthesia with $2-3 \%$ isoflurane delivered via nose cone. The colon was removed by cutting away mesenteric attachments and excising the tissue from caecum to rectum. Colons were immediately flushed with ice-cold modified Krebs solution containing the following, in mM: $140 \mathrm{Na}^{+}, 5.2 \mathrm{~K}^{+}, 1.2 \mathrm{Ca}^{2+}, 1.2 \mathrm{Mg}^{2+}, 119.8 \mathrm{Cl}^{-}, 25 \mathrm{HCO}_{3}{ }^{-}, 2.4 \mathrm{HPO}_{4}^{2-}, 0.4$ $\mathrm{H}_{2} \mathrm{PO}_{4}{ }^{-}$, and 10 glucose. Krebs solution was bubbled with $5 \% \mathrm{CO}_{2}$ balanced with oxygen and the was adjusted to $\mathrm{pH}$ 7.41. After removal of the colon, rats were euthanized by cutting the diaphragm and the colon was used for down-stream Ussing chamber, immunofluorescence, or Western blotting experiments. For some experiments, the ileum was also removed and flushed in the same manner. All experimental procedures were approved by West Virginia University Animal Care and Use Committee.

Previous work in our lab regarding the effects of $\mathrm{K}_{\mathrm{V}} 7$ activation in the ENS has been conducted using mice. In the present study, rats were chosen as the primary model system for several reasons: 1) to establish that the effect(s) of $\mathrm{K}_{\mathrm{V}} 7$ activation on colonic epithelial transport are not unique to the mouse, 2) to allow for more substantiated interpretation of the findings regarding the modulation of $\mathrm{Na}^{+}$transport, as much of the previous work in field of colonic $\mathrm{Na}^{+}$ transport has been conducted in rats $(23,33,34,35,36)$, and 3$)$ to minimize the number of required animals, as the larger surface area of the rat colon permits the use of multiple tissue segments per animal for unidirectional flux experiments. In addition, only male rats were used in this study 
because many aspects of colonic ion transport are markedly different in females versus males, likely owing to modulation by sex steroids $(37,38,39)$. Future studies will be aimed at exploring any sex differences in the response to flupirtine or other ENS-modulating drugs.

\section{Ussing chamber experiments}

After flushing, the colon was opened longitudinally along the mesenteric border and placed mucosal side down in a dissecting dish, submerged in ice-cold bubbled Krebs solution. The serosa, longitudinal and circular smooth muscle layers were gently stripped away first by making a lateral incision at the junction between proximal and distal segments (i.e., at the distal end of the proximal striations). Muscle and serosal layers were then peeled back using a glass slide and fine forceps to obtain a mucosa/muscularis mucosae/submucosa preparation. Tissues prepared in this way contain functional submucosal neurons, but lack the myenteric plexus altogether. Colonic segments were then mounted on $1.1 \mathrm{~cm}^{2}$ sliders with a circular aperture for use in an Ussing-style recording chamber (Physiologic Instruments, San Diego, CA). Mounted tissues were bathed bilaterally in $5 \mathrm{~mL}$ of cold Krebs buffer, bubbled with $5 \% \mathrm{CO}_{2}$ balanced with oxygen and gradually warmed to $37^{\circ} \mathrm{C}$. For experiments using proximal colon, the tissue was prepared the same way as described here using segments of proximal colon characterized by striation patterns. For experiments using ileum, tissues were partially stripped and mounted on $0.3 \mathrm{~cm}^{2}$ oval aperture sliders. This difference in slider aperture was corrected for in the electrical recordings and $\mathrm{Na}^{+}$flux calculations described below.

Chambers were equipped with a pair voltage-sensing $\mathrm{AgCl}$ pellet electrodes and currentinjecting $\mathrm{AgCl}$ wire electrodes connected to the chamber bath via agar-salt bridge $3.5 \%$ agar in $3 \mathrm{M} \mathrm{KCl}$ ) embedded in the electrode tips. After mounting, tissues were allowed $\sim 15$ minutes to 
equilibrate and be brought up to temperature under open circuit conditions, during which time trans-epithelial potential $\left(V_{T E}\right)$ was monitored. Tissues were then voltage-clamped to $0 \mathrm{mV}$ using a multi-channel voltage clamp/amplifier (VCC MC8; Physiologic Instruments) and short-circuit current $\left(I_{S C}\right)$ was continuously monitored by automated computer software (Acquire \& Analyze; Physiologic Instruments). $5 \mathrm{mV}, 200 \mathrm{~ms}$ bipolar pulses were applied at $30 \mathrm{sec}$ intervals to monitor trans-epithelial conductance $\left(G_{T E}\right)$. By convention, positive $\mathrm{I}_{\mathrm{SC}}$ values reflect net anion $\left(\mathrm{Cl}^{-} / \mathrm{HCO}_{3}^{-}\right.$ ) secretion and/or cation $\left(\mathrm{Na}^{+}\right)$absorption; negative $\mathrm{I}_{\mathrm{SC}}$ values reflect net anion absorption or cation $\left(\mathrm{K}^{+}\right)$secretion.

Unidirectional ${ }^{22} \mathrm{Na}^{+}$fluxes were performed by first assigning tissue pairs with $\mathrm{G}_{\mathrm{TE}}$ values within $15 \%$ of each other. $2 \mu \mathrm{Ci}$ of ${ }^{22} \mathrm{Na}^{+}$was then added to either the serosal (basolateral) or mucosal (apical) chamber bath and allowed 25 minutes to reach a steady-state rate of flux. $250 \mu \mathrm{L}$ samples were taken in duplicate from the opposite ("cold") chamber bath at the beginning and end of each 30-minute flux period and replaced with $500 \mu \mathrm{L}$ of fresh buffer. Drugs were added as 1000x stock solutions, administered in $5 \mu \mathrm{L}$ volumes to the $5 \mathrm{~mL}$ chamber bath immediately following sample collection. Drug concentration and placement details are given in the figure legends. At the end of each experiment, $50 \mu \mathrm{L}$ samples were taken from the "hot" chamber bath (where ${ }^{22} \mathrm{Na}^{+}$tracer was added) to obtain a standard value used in the calculation of $\mathrm{Na}^{+}$flux rate. Samples were then mixed with liquid scintillation cocktail (Sigma Aldrich, \#03999-5L) and counted using a Tri-carb 4910TR liquid scintillation counter (Perkin Elmer, Waltham MA). Unidirectional ${ }^{22} \mathrm{Na}^{+}$flux rates were calculated from the measured counts per minute (cpm) values using the following equation:

$$
N a^{+} \text {flux }(J)\left(\mu E q / \mathrm{cm}^{2} \cdot h r\right)=(5 \times B-[A \times 0.9]) /([S / 140] \times 1.1 \times 0.5)
$$


"A" and "B" represent $\mathrm{cpm} / \mathrm{mL}$ values measured from the beginning and end of a flux period, respectively. 5 is the total volume (in $\mathrm{mL}$ ) of the chamber bath and 0.9 is dilution factor accounting for removal of $500 \mu \mathrm{L}$ of bath volume upon sample collection. "S" represents the $\mathrm{cpm} / \mu \mathrm{Eq}$ (in $1 \mathrm{~mL}$ ) measured from the standard obtained from the "hot" bath of the chamber. 1.1 corresponds to the slider aperture (in $\mathrm{cm}^{2}$ ) and 0.5 is the time (in hours) for each flux period (40). Net $\mathrm{Na}^{+}$fluxes were calculated by subtracting the serosal to mucosal $(S-M)$ from the mucosal to serosal $(M-S)$ fluxes from paired tissues for a given flux period.

\section{Immunofluorescence microscopy}

To identify the presence of flupirtine-sensitive $\mathrm{K}_{\mathrm{V}} 7$ channels in submucosal neurons, segments of mid-distal colon were dissected under a microscope to obtain a submucosal preparation, as described previously (14). First, the colon was opened longitudinally and pinned mucosal side down on a dissecting stage. A shallow lateral incision was made near the end of the striations of the proximal colon to transect the longitudinal and circular smooth muscle layers, which were then peeled away in a distal direction until completely removed. The tissue was then inverted and pinned mucosal side up. Another lateral incision was made near the rectum, just deep enough to isolate the mucosa/muscularis mucosae from the underlying submucosal layer. The mucosal/muscularis mucosae was removed by carefully creating a plane of separation using ultrafine forceps, and then gently rubbing in a proximal direction with the index finger. The remaining submucosal layer was immediately fixed on $4 \%$ paraformaldehyde (PFA) dissolved in phosphate buffered saline (PBS) overnight at $4^{\circ} \mathrm{C}$.

The following day, the tissue was permeabilized with PBS $+1 \%$ Triton- $X+5 \%$ goat serum

for 1 hour at room temperature and blocked for an additional 30 minutes in PBS $+0.01 \%$ Triton- 
$\mathrm{X}(\mathrm{PBST})+5 \%$ goat serum. The tissue was then incubated in PBST $+5 \%$ goat serum containing the primary antibodies: mouse anti-microtubule associated protein 2 (MAP-2; neuronal marker) (Millipore \#MAB3418; 1:200 dilution) (41) and rabbit anti-KCNQ2 (flupirtine-sensitive $\mathrm{K}_{\mathrm{V}} 7$ channel subtype) (Thermo \#PA1-929; 1:100 dilution) (20) overnight at $4^{\circ} \mathrm{C}$. The next day, the tissue was washed 5 times for 5 minutes each in PBST before incubation in PBST $+5 \%$ goat serum containing AlexaFluor 488 goat anti-rabbit (Thermo \#A11008; 1:2,000 dilution) and AlexaFluor 568 goat-anti-mouse (Thermo \#A11031; 1:2,000 dilution) for 30 minutes at room temperature, after which it was again washed 5 times for 5 minutes each in PBST. The tissue was then rinsed briefly in water and placed flat on a charged glass microscope slide. SlowFade Diamond mountant with DAPI (Thermo \#36971) was used to mount a coverslip on the slide, which was then sealed with clear fingernail polish. Images were captured using a Zeiss 710 confocal microscope under 20x magnification (Carl Zeiss, Oberkochen, Germany).

For immunofluorescence experiments to visualize NHE-3 localization in the epithelium, intact (unopened) colons were stripped of longitudinal and circular smooth muscle and incubated in bubbled Krebs solution with or without flupirtine for 10 minutes at $37^{\circ} \mathrm{C}$. The tissues were then immediately fixed in pre-warmed 4\% PFA in PBS for 15 minutes. After washing 3 times in cold PBS, tissues were flash frozen in isopentane cooled with liquid nitrogen and embedded in tissue freezing medium (Scigen \#4586, Gardena, CA). $10 \mu \mathrm{m}$ sections were cut with a cryostat and mounted on charged glass slides. Sections were permeabilized in PBS $+0.1 \%$ Triton- $X+5 \%$ goat serum for 20 minutes at room temperature, washed briefly in PBST and blocked in PBST $+5 \%$ goat serum for an additional 30 minutes. Next, sections were incubated in PBST $+5 \%$ goat serum containing NHE-3-specific primary antibody (Abcam \#95299; 1:100 dilution) (39) for 1 hour at $37^{\circ} \mathrm{C}$ in a humidifying chamber. The sections were subsequently washed 5 times or 5 minutes 
each and incubated in PBST $+5 \%$ goat serum containing AlexaFluor 488 goat anti-rabbit (Thermo \#A11008; 1:2,000 dilution) and TRITC-conjugated phalloidin (filamentous actin labelling) (Thermo \#R415; 1:1,000 dilution) for 30 minutes at room temperature. Finally, sections were washed again 5 times for 5 minutes each and mounted using SlowFade Diamond mountant with DAPI (Thermo \#36971). Slides were sealed with clear fingernail polish and imaged using a Zeiss 710 confocal microscope under 20x magnification (Carl Zeiss, Oberkochen, Germany).

\section{Western blotting}

Colons from normal or dietary $\mathrm{Na}^{+}$-depleted rats were flushed with ice-cold Krebs solution to remove fecal contents. A clamp was then placed on the distal end of the colon, near the rectum, and the colons were filled with ice-cold epithelial isolation solution containing the following, in mM: $30 \mathrm{NaCl}, 8$ HEPES-Tris ( $\mathrm{pH}$ 7.5), 5 ethylenediaminetetraacetic acid (EDTA), 1 phenylmethylsulphonyl fluoride (PMSF), and 0.5 dithiothreitol (DTT). After filling, the colons were secured on the proximal end with a small ligature and incubated in this same solution on ice for 30 minutes. Then the colons were emptied, opened longitudinally along the mesenteric border and scraped with a glass slide to remove the mucosa, which was flash frozen in liquid nitrogen and stored at $-80^{\circ} \mathrm{C}$ until use.

Proteins were extracted from the tissues by homogenizing in ice-cold radioimmunoprecipitation assay (RIPA) lysis buffer and briefly sonicating to disrupt membranes. Samples were centrifuged for 20 minutes at 12,000 x g and the supernatants were collected and mixed with $4 \mathrm{x}$ Laemmli buffer. The samples were then heated to $90^{\circ} \mathrm{C}$ for 5 minutes and immediately chilled on ice before adding 2-mercaptoethanol $(5 \% \mathrm{w} / \mathrm{v})$. Proteins were resolved on $8 \%$ polyacrylamide gels and transferred to PVDF membranes, which were subsequently blocked 
in 3\% bovine serum albumin (BSA) in tris-buffered saline plus $0.1 \%$ Tween-20 (TBST) for 1 hour at room temperature. Membranes were then incubated overnight in TBST plus 3\% BSA solution containing rabbit anti-NHE-3 (Abcam \#95299; 1:500 dilution) or horseradish peroxidase (HRP)conjugated mouse anti- $\beta$-actin (SCBT \#SC-47778; 1:1,000 dilution) primary antibody. The following day, membranes were washed in TBST 5 times for 5 minutes each. Membranes that were probed for NHE-3 were then incubated in HRP-conjugated goat anti-rabbit secondary antibody (Thermo \#31462) for 1 hour at room temperature and subsequently washed an additional 5 times for 5 minutes each. Immune complexes were detected via chemiluminescence using West Dura extended duration substrate (Thermo \#34075) and imaged on a G-box. Protein band intensity was quantified using FIJI (Image J) software.

\section{Drugs and chemicals}

Flupirtine, vasoactive intestinal polypeptide (VIP) and tetrodotoxin were purchased from Tocris (Minneapolis, MN). Veratridine was purchased from Alomone labs (Jerusalem, IL). Amiloride and S3226 were purchased from Sigma Aldrich (St. Louis, MO). HOE694 was a generous gift from Dr. Selvi Krishnan. All other chemicals and salts were purchased either from Sigma Aldrich or Fisher Scientific (Waltham, MA).

\section{Data analysis}

$I_{\mathrm{SC}}$ and $\mathrm{G}_{\mathrm{TE}}$ values were averaged from each tissue over the final 5 minutes for a given flux period when used for comparison between treatment groups or values obtained from consecutive flux periods unless otherwise noted. Duplicate cpm values were averaged for use in the calculation of unidirectional ${ }^{22} \mathrm{Na}^{+}$flux for a given 30-minute period. Evaluation of statistical significance was determined using GraphPad Prism 9 software, via unpaired Student's t-test for comparison 
between two treatment groups/flux periods, or via one-way analysis of variance (ANOVA) with Tukey's post-hoc when comparing more than two treatment groups/flux periods. Statistical test details are given in the figure legends. For correlation analysis of change in $\mathrm{I}_{\mathrm{SC}}\left(\Delta \mathrm{I}_{\mathrm{SC}}\right)$ versus change in M-S Na ${ }^{+}\left(\Delta \mathrm{J}_{\mathrm{M}-\mathrm{s}}\right)$ flux, Pearson's $r$ coefficient was determined using product-moment correlation analysis in GraphPad Prism 9 software. Statistical significance was defined as $p<0.05$ for all analyses.

\section{RESULTS}

$K_{V} 7$ channel activator, flupirtine, attenuates neurogenic secretion in rat distal colon.

Previously studies in our lab have shown that electrogenic $\mathrm{Cl}^{-}$secretion (measured as $\mathrm{I}_{\mathrm{SC}}$ ) was dramatically inhibited in mouse colon by the application of flupirtine to the serosal side of the tissue. Pre-treatment with flupirtine also blunted or abolished the effects of neural-targeted secretory agents, veratridine ( $\mathrm{Na}_{\mathrm{v}}$ channel opener) and nicotine (nAChR agonist), while sparing the effects of the epithelium-targeted secretagogues VIP (VPAC receptor agonist) and carbachol (non-selective cholinergic agonist). Figure 1 demonstrates that a similar pattern is observed in the rat colon. Segments of mid-distal colon pre-treated with flupirtine $(100 \mu \mathrm{M})$ exhibited markedly reduced basal $\mathrm{I}_{\mathrm{SC}}$ compared to control (untreated vs. FLU: $18.6 \pm 1.2$ vs. $121.2 \pm 11.6 \mu \mathrm{A} / \mathrm{cm}^{2}$; $p<0.01$ ) (Fig. 1A-B). Flupirtine-treated tissues responded to serosal VIP (untreated vs. FLU: $\Delta \mathrm{I}_{\mathrm{SC}(\mathrm{VIP})}=84.1 \pm 16.0$ vs. $94.1 \pm 15.8 \mu \mathrm{A} / \mathrm{cm}^{2} ; p=0.67$ ) (Fig. 1D), but displayed a significantly diminished response to serosal veratridine (untreated vs. FLU: $\Delta \mathrm{I}_{\mathrm{SC}(\mathrm{VER})}=69.5 \pm 11.5$ vs. $24.9 \pm$ $4.0 \mu \mathrm{A} / \mathrm{cm}^{2} ; p<0.01$ ) (Fig. 1C). Labelling of $\mathrm{K}_{\mathrm{V}} 7.2$ in MAP-2 ${ }^{+}$cells of rat submucosal ganglia (Fig. 1E) confirmed the presence of flupirtine-sensitive channels in these tissues, consistent with those observed in mouse. These preliminary studies confirm that the effects of flupirtine are similar 
between mouse and rat with respect to neurogenic secretion and support the use of the rat colon as a valid model for studying the effects flupirtine on $\mathrm{Na}^{+}$transport in the experiments to follow.

Flupirtine enhances $\mathrm{Na}^{+}$absorption in rat distal colon in an ENS-dependent manner.

After establishing that the effects of flupirtine are consistent between mouse and rat regarding neurogenic $\mathrm{Cl}^{-}$secretion, ${ }^{22} \mathrm{Na}^{+}$flux experiments were performed next to determine whether epithelial $\mathrm{Na}^{+}$transport was also altered by flupirtine. For initial experiments, colonic tissues were allowed to equilibrate for 15 minutes with or without flupirtine in the serosal bath, prior to the addition of isotope. After isotope equilibration (25 minutes), unidirectional and net $\mathrm{Na}^{+}$fluxes were calculated for the subsequent 30-minute flux period as described above. $\mathrm{Na}^{+}$ fluxes, as well as $\mathrm{I}_{\mathrm{SC}}$ and $\mathrm{G}_{\mathrm{TE}}$ data are listed in Table 1. Under these conditions, flupirtine enhanced net $\mathrm{Na}^{+}$absorption by $\sim 75 \%$ (untreated vs. FLU: $6.6 \pm 1.2$ vs $11.7 \pm 1.2 \mu \mathrm{Eq} / \mathrm{cm}^{2} \cdot \mathrm{hr} ; p<0.05$ ) via a significant increase in the mucosal-to-serosal (M-S) flux ( $p<0.05)$, as well as a slight decrease in serosal-to-mucosal $(\mathrm{S}-\mathrm{M})$, which was not statistically significant $(p=0.148)$. At the same time, basal $\mathrm{I}_{\mathrm{SC}}$ and $\mathrm{G}_{\mathrm{TE}}$ were both reduced in flupirtine-treated tissues ( $p<0.01$ for both).

Next, experiments were designed to determine whether the enhancement of $\mathrm{Na}^{+}$absorption by flupirtine was mediated through effects on the ENS, as is the case for the inhibition of epithelial $\mathrm{Cl}^{-}$secretion. To test this, $\mathrm{Na}^{+}$fluxes were measured in sequential 30-minute periods, before and after application of flupirtine, using tissues that were either untreated (control) or pre-treated with $1 \mu \mathrm{M}$ tetrodotoxin $\left(\mathrm{Na}_{\mathrm{V}}\right.$ channel blocker) to abolish ENS activity $(12,42)$. Flupirtine-sensitive $\mathrm{I}_{\mathrm{SC}}$ was much greater in the absence versus in the presence of tetrodotoxin (control vs. TTX: $\Delta \mathrm{I}_{\mathrm{SCC} \text { (FL) }}$ $=-92.8 \pm 11.4$ vs. $-9.5 \pm 2.6 \mu \mathrm{A} / \mathrm{cm}^{2} ; p<0.01$ ) (Fig. $2 \mathrm{C}$ ), as was flupirtine-sensitive $\mathrm{G}_{\mathrm{TE}}$ (control vs. TTX: $\Delta \mathrm{G}_{\mathrm{TE}(\mathrm{FLU})}=-2.9 \pm 0.5$ vs. $-1.0 \pm 0.1 \mathrm{mS} / \mathrm{cm}^{2} ; p<0.01$ ) (Fig. $2 \mathrm{D}$ ). These results mirror 
those described in mouse. $\mathrm{Na}^{+}$absorption was acutely enhanced by flupirtine in control tissues (basal vs. FLU: $2.4 \pm 1.0$ vs $\left.7.8 \pm 0.8 \mu \mathrm{Eq} / \mathrm{cm}^{2} \cdot \mathrm{hr} ; p<0.05\right)$ via an increase in M-S flux $(p<0.01)$ and a slight decrease in S-M flux $(p<0.05)$. However, pre-treatment with tetrodotoxin negated this effect (basal vs. FLU: $10.4 \pm 1.6$ vs $9.9 \pm 0.4 \mu \mathrm{Eq} / \mathrm{cm}^{2} \cdot \mathrm{hr} ; p=0.55$ ). Flupirtine also reduced $\mathrm{I}_{\mathrm{SC}}$ and $\mathrm{G}_{\mathrm{TE}}$ significantly in both control and tetrodotoxin-treated tissues $(p<0.01$ for all), although the change from baseline was greater in control tissues, as basal $\mathrm{I}_{\mathrm{SC}}$ and $\mathrm{G}_{\mathrm{TE}}$ values were higher in the absence of ENS blockade via tetrodotoxin. Table 2 provides the details of $\mathrm{Na}^{+}$fluxes, $\mathrm{I}_{\mathrm{SC}}$ and $\mathrm{G}_{\mathrm{TE}}$ data from these experiments.

Figure $3 \mathrm{~A}$ summarizes the net $\mathrm{Na}^{+}$absorption measured before and after flupirtine treatment in the presence and absence of tetrodotoxin. When the net flux values were compared to each other via one-way ANOVA with Tukey's post-hoc, a significant increase was again observed only in control tissues (pre- vs. post-flupirtine; $p<0.01$ ), but not tetrodotoxin-treated tissues (prevs. post flupirtine; $p>0.97)$. In addition, net $\mathrm{Na}^{+}$absorption was significantly greater in both tetrodotoxin- and tetrodotoxin/flupirtine-treated tissues compared to the basal flux for control $\left(p<0.01\right.$ for both). However, there was not a significant difference in $\mathrm{Na}^{+}$flux between control tissues post-flupirtine and tetrodotoxin-treated tissue either before or after flupirtine application $(p=0.11$ and 0.23 , respectively).

In these experiments, ${ }^{22} \mathrm{Na}^{+}$fluxes were measured in sequential 30-minute periods, before and after administration of flupirtine. This differs from the experiments summarized in Table 1, where fluxes were time-matched and measured either in the presence or absence of flupirtine. The additional equilibration time at the beginning of those experiments may explain the relatively higher rates of net $\mathrm{Na}^{+}$flux, as more time had passed before the addition of isotope. For additional comparison, time-matched $\mathrm{Na}^{+}$fluxes were compiled from both sets of experiments (Tables 1 and 
2) and are depicted in Figure 3B. When compared via one-way ANOVA with Tukey's post-hoc, $\mathrm{Na}^{+}$absorption was again significantly greater in flupirtine-treated tissues $(p<0.05)$, while no difference was observed between treatment with flupirtine and tetrodotoxin $(p=0.799)$. Although not statistically significant $(p=0.076), \mathrm{Na}^{+}$absorption was also greater in tetrodotoxin-treated colon compared to control. These studies demonstrate that the effects of flupirtine on $\mathrm{Na}^{+}$absorption likely occur via a tetrodotoxin-sensitive mechanism (i.e., by acting on ENS neurons), consistent with the hypothesis that inhibition of neurogenic input via activation of neuronal $\mathrm{K}_{\mathrm{V}} 7$ channels both dampens epithelial secretion enhances $\mathrm{Na}^{+}$absorption.

Flupirtine enhances $\mathrm{Na}^{+}$absorption that is primarily mediated by NHE-3.

Increased $\mathrm{Na}^{+}$absorption without an accompanying increase in $\mathrm{I}_{\mathrm{SC}}$ indicates that flupirtine enhanced $\mathrm{Na}^{+}$flux through an electroneutral absorptive pathway. $\mathrm{Na}^{+} / \mathrm{H}^{+}$exchanger isoform 3 (NHE-3) is the predominant electroneutral $\mathrm{Na}^{+}$transporter within the intestinal mucosa (29). Importantly, NHE-3 is also known to be regulated by intracellular signaling events occurring in direct response to ENS stimulation, such as an increase in cytosolic $\mathrm{Ca}^{2+}$ or cAMP following cholinergic or VIP-ergic input (12). However, $\mathrm{Na}^{+} / \mathrm{H}^{+}$exchanger isoform 2 (NHE-2) activity has also been reported to play an important role in electroneutral $\mathrm{Na}^{+}$absorption under certain conditions as well $(43,44,45)$. Studies were therefore initiated to evaluate the relative contributions of NHE-2 and NHE-3 transporters in flupirtine-stimulated $\mathrm{Na}^{+}$absorption.

$\mathrm{Na}^{+}$fluxes were measured in the presence of flupirtine alone (control) or in the presence of $50 \mu \mathrm{M}$ HOE694 (NHE-2 selective inhibitor) (45), $10 \mu \mathrm{M}$ S3226 (NHE-3 selective inhibitor) (46) or $1 \mathrm{mM}$ amiloride (non-selective inhibitor of electroneutral/electrogenic $\mathrm{Na}^{+}$absorption) (47) in the mucosal bath. As illustrated in Figure 4, mucosal HOE694 had no discernable effect (FLU 
vs. HOE694: $10.5 \pm 0.4$ vs $\left.10.0 \pm 0.5 \mu \mathrm{Eq} / \mathrm{cm}^{2} \cdot \mathrm{hr} ; p=0.55\right) .(p=0.98)$. However, net $\mathrm{Na}^{+}$absorption was substantially inhibited by both S3226 (FLU vs. S3226: $10.5 \pm 0.4$ vs $4.2 \pm 0.3 \mu \mathrm{Eq} / \mathrm{cm}^{2} \cdot \mathrm{hr}$; $p<0.01$ ) and amiloride (FLU vs. AMIL: $10.5 \pm 0.4$ vs $1.9 \pm 0.5 \mu \mathrm{Eq} / \mathrm{cm}^{2} \cdot \mathrm{hr} ; p<0.01$ ), owing to a large decrease in M-S flux (details given in Table 3). Amiloride inhibited $\mathrm{Na}^{+}$absorption to a greater extent than S3226 amiloride (S3226 vs. AMIL: $4.2 \pm 0.3$ vs $1.9 \pm 0.5 \mu \mathrm{Eq} / \mathrm{cm}^{2} \cdot h r ; p<0.01$ ), also a result of reduced M-S flux (see Table 3), indicating that a concentration of $10 \mu \mathrm{M} \mathrm{S3226}$ may not be sufficient to maximally inhibit NHE-3 under these conditions. Nonetheless, S3226 abolished the majority $(\sim 70 \%)$ of amiloride-sensitive $\mathrm{Na}^{+}$absorption in the presence of flupirtine, suggesting critical involvement of NHE-3 activity in this process. These observations fit nicely with the current understanding of NHE-3 regulation by the ENS, as the apparent suppression of ENS activity via $\mathrm{K}_{\mathrm{V}} 7$ activation triggers a considerable increase in NHE-3-dependent absorption.

Extensive characterization of acute NHE-3 regulation in the intestine has revealed a primary mechanism involving rapid changes in membrane localization through trafficking between the apical membrane and an intracellular vesicular pool (48). Stimulation of various basolateral receptors, such as $\mathrm{M}_{3} \mathrm{R}$ via acetylcholine, triggers the internalization of NHE-3, thus inhibiting $\mathrm{Na}^{+}$uptake across the apical membrane. If activation of ENS-localized $\mathrm{K}_{\mathrm{V}} 7$ channels via flupirtine causes reduced stimulatory neurogenic input that is associated with enhanced NHE3-dependent $\mathrm{Na}^{+}$absorption (Figs 1-4), then NHE-3 trafficking is likely to play a role in this process. Qualitatively, seromuscular-stripped rat distal colon that was treated with flupirtine appeared to have more NHE-3 protein localized near the apical membrane (labelled with phalloidin) compared with control (Figure 5). The NHE-3-specific labelling was more diffuse beneath the apical surface in untreated control tissue, while appearing more focused around the apical membrane in flupirtine-treated tissue. While quantitative analysis was not performed here, 
the apparent change in NHE-3 localization in response to flupirtine-mediated suppression of ENS activity is congruent with the results from our Ussing chamber experiments, as well as with the currently accepted model of acute NHE-3 regulation in colonic epithelia.

Flupirtine does not enhance ENaC-mediated $\mathrm{Na}^{+}$absorption in hyperaldosteronaemic rat distal colon.

Both electroneutral and (NHE-2/3-mediated) and electrogenic (ENaC-mediated) $\mathrm{Na}^{+}$ absorption are present in the colon of most mammalian species, but there is considerable variability in the functional importance, as well as the anatomical distribution of these pathways (43). Along with this, second messenger systems responding to ENS-derived neurotransmitters, such as $\mathrm{Ca}^{2+}$ $\left(\mathrm{M}_{3} \mathrm{R}\right.$ activation) or cAMP (VPAC1 activation), differentially regulate NHE-3 and ENaC as well. For example, cAMP is known rapidly inhibit NHE-3 activity, whereas $\mathrm{ENaC}$ has been shown to be activated by elevated cAMP $(49,50)$ and its expression may be governed by $\mathrm{Ca}^{2+}$ events $(51)$. Beyond this, there has been little work done to elucidate the regulation of ENaC by ENS neurotransmission, specifically, within the colon.

The data presented thus far support the notion that activation of $\mathrm{K}_{\mathrm{V}} 7$ channels in the ENS augments NHE-3-mediated absorption. However, the potential effects of $\mathrm{K}_{\mathrm{V}} 7$ activation on $\mathrm{ENaC}$ mediated electrogenic absorption remain undetermined. $\mathrm{Na}^{+}$absorption in the normal rat distal colon is almost exclusively electroneutral with very little involvement of ENaC-mediated electrogenic $\mathrm{Na}^{+}$absorption, except for the most distal segment of the colon and rectum (4). To address this problem, the effect of flupirtine on $\mathrm{Na}^{+}$flux was measured in distal colon of dietary $\mathrm{Na}^{+}$-depleted rats, which exhibit secondary hyperaldosteronism (30). One of the hallmark 
characteristics of this model is the induction of electrogenic (ENaC-mediated) $\mathrm{Na}^{+}$absorption and the concurrent down-regulation of NHE-3, as well as NHE-2 (43).

Basal ISC is typically much higher (2- to 4-fold) in the distal colon of hyperaldosteronaemic (a.k.a. "aldo") rats compared to normal controls, which is attributable mostly to ENaC activity. In "aldo" distal colon, addition of flupirtine to the serosal bath caused a reduction in ISC $_{\text {(basal vs. }}$ FLU: $297.8 \pm 7.2$ vs. $193.5 \pm 11.8 \mu \mathrm{A} / \mathrm{cm}^{2} ; p<0.01$ ) and $\mathrm{G}_{\mathrm{TE}}$ (basal vs. FLU: $11.8 \pm 0.7$ vs. $7.4 \pm$ $0.3 \mathrm{mS} / \mathrm{cm}^{2} ; p<0.01$ ) (Fig. 5A-B). However, this inhibition appears qualitatively to be more gradual in onset when compared the $\mathrm{I}_{\mathrm{SC}}$ inhibition observed in normal rats. $\mathrm{I}_{\mathrm{SC}}$ declined steadily over the 30-minute flux period in "aldo" tissues, whereas ISC in controls reached a new baseline within a few minutes (see Fig. 2). $\mathrm{Net} \mathrm{Na}^{+}$flux was unaffected by flupirtine in "aldo" distal colon (basal vs. FLU: $11.4 \pm 0.7$ vs $10.6 \pm 0.8 \mu \mathrm{Eq} / \mathrm{cm}^{2} \cdot \mathrm{hr} ; p=0.66$ ) (Fig. $5 \mathrm{C}$ ), despite the concurrent reduction in $\mathrm{I}_{\mathrm{SC}}$ and $\mathrm{G}_{\mathrm{TE}}$, suggesting a portion of the basal $\mathrm{I}_{\mathrm{SC}}$ was not mediated by electrogenic $\mathrm{Na}^{+}$transport (e.g., $\mathrm{Cl}^{-} / \mathrm{HCO}_{3}{ }^{-}$secretion). However, subsequent application of $10 \mu \mathrm{M}$ amiloride (ENaC-selective at this concentration) (52) dramatically inhibited ISC (FLU vs. AMIL: $193.5 \pm$ 11.8 vs. $\left.-27.9 \pm 2.5 \mu \mathrm{A} / \mathrm{cm}^{2} ; p<0.01\right)$ and $\mathrm{G}_{\mathrm{TE}}$ (FLU vs. AMIL: $7.4 \pm 0.3$ vs. $5.0 \pm 0.2 \mathrm{mS} / \mathrm{cm}^{2}$; $p<0.01$ ) (Fig. 5A-B), as well as $\mathrm{Na}^{+}$absorption (FLU vs. AMIL: $10.6 \pm 0.8$ vs $2.0 \pm 0.3 \mu \mathrm{Eq} / \mathrm{cm}^{2} \cdot \mathrm{hr}$; $p<0.01$ ) (Fig. 5C), due to a substantial reduction in M-S flux $(p<0.01)$.

The profound effects of low-dose amiloride on $\mathrm{I}_{\mathrm{SC}}, \mathrm{G}_{\mathrm{TE}}$ and $\mathrm{Na}^{+}$flux indicate the prominent activity of $\mathrm{ENaC}$ in the distal colon of these animals. In addition, Western blot analysis using distal colonic mucosae from normal and "aldo" rats confirmed that NHE-3 expression was significantly down-regulated ( $<25 \%$ of control), as shown in the representative blot image (Fig. 5D) and densitometric quantification (Fig. 5E). Based on these observations, activation of $\mathrm{K}_{\mathrm{V}} 7$ channels in 
the ENS may thus only enhance electroneutral NHE-3-mediated absorption in rat colon, as ENaCmediated absorption is already quite high at baseline, and not altered by treatment with flupirtine.

Effects of flupirtine on $\mathrm{Cl}^{-}$secretion and $\mathrm{Na}^{+}$absorption are specific to the colon.

The results described above suggest that enhanced $\mathrm{Na}^{+}$absorption in response to $\mathrm{K}_{\mathrm{V}} 7$ activation was mediated only by electroneutral, NHE-3-mediated transport. Indeed, NHE-3 is the major $\mathrm{Na}^{+}$absorptive mechanism along the length of the small and large intestine. The compositional make-up of submucosal ENS neuronal networks is widely varied between anatomical regions, however. Decades of investigation into the neurochemical coding of GI innervation has helped to characterize the various ENS secretomotor subpopulations. The spatial and functional configuration of the major neuronal subtypes (e.g., cholinergic, VIP-ergic, nitrergic) has been fairly well-defined (8). Still undetermined is whether segmental differences exist regarding the response to flupirtine on either $\mathrm{Cl}^{-}$secretion $\left(\mathrm{I}_{\mathrm{SC}}\right)$ or $\mathrm{Na}^{+}$absorption, as the specific distribution pattern of $\mathrm{K}_{\mathrm{V}} 7$ channel subtypes has not been described in these networks to date. Thus, the effects of $\mathrm{KV}_{\mathrm{V}} 7$ activation on $\mathrm{Cl}^{-}$secretion and $\mathrm{Na}^{+}$absorption were assessed in both the proximal colon and the ileum to determine whether the response was region-specific.

Flupirtine inhibited $\mathrm{I}_{\mathrm{SC}}$ (basal vs. FLU: $70.7 \pm 14.2$ vs. $25.8 \pm 2.2 \mu \mathrm{A} / \mathrm{cm}^{2} ; p<0.01$ ) and $\mathrm{G}_{\mathrm{TE}}$ (basal vs. FLU: $9.7 \pm 0.5$ vs. $7.6 \pm 0.4 \mathrm{mS} / \mathrm{cm}^{2} ; p<0.01$ ) (Fig. $6 \mathrm{~A}, \mathrm{C}$ ), while stimulating $\mathrm{Na}^{+}$ absorption (basal vs. FLU: $3.1 \pm 0.8$ vs $5.7 \pm 0.6 \mu \mathrm{Eq} / \mathrm{cm}^{2} \cdot \mathrm{hr} ; p<0.05$ ) (Fig. $6 \mathrm{E}$ ) in rat proximal colon, suggesting the presence of flupirtine-sensitive $\mathrm{K}_{\mathrm{V}} 7$ channels in this region. Flupirtinestimulated $\mathrm{Na}^{+}$absorption was also inhibited by mucosal S3226 (FLU vs. S3226: $5.7 \pm 0.6$ vs 2.8 $\pm 0.2 \mu \mathrm{Eq} / \mathrm{cm}^{2} \cdot \mathrm{hr} ; p<0.01$ ) (not illustrated), suggesting that NHE-3 is the primary mediator in the proximal colon as well. In the ileum, however, flupirtine did not inhibit $\mathrm{I}_{\mathrm{SC}}$ (basal vs. FLU: $96.2 \pm$ 
6.0 vs. $97.9 \pm 6.4 \mu \mathrm{A} / \mathrm{cm}^{2} ; p=0.84$ ), slightly increased $\mathrm{G}_{\mathrm{TE}}$ (basal vs. FLU: $23.9 \pm 0.9$ vs. $26.7 \pm$ $0.8 \mathrm{mS} / \mathrm{cm}^{2} ; p<0.05$ ) (Fig. 6B,D) and did not alter $\mathrm{Na}^{+}$absorption (basal vs. FLU: $4.9 \pm 0.6$ vs 5.7 $\left.\pm 0.7 \mu \mathrm{Eq} / \mathrm{cm}^{2} \cdot \mathrm{hr} ; p=0.41\right)($ Fig. $6 \mathrm{~F})$. Together, these data indicate the effects of $\mathrm{K}_{\mathrm{V}} 7$ channel activation in ENS neurons may be specific to the colon, at least in rats.

Stimulation of $\mathrm{Na}^{+}$absorption and inhibition of $\mathrm{Cl}^{-}$secretion are significantly correlated in flupirtine treated rat distal colon.

Release of ENS-derived neurotransmitters to the intestinal epithelium has effects on both secretion and absorption within cells of the crypt and surface regions, respectively (7). By inducing secretion and inhibiting absorption together in a coordinated manner, ENS activity can produce a flushing secretory response that is often coupled to enhanced motility for the purpose of removing potentially harmful substances from the bowel. However, this response is often inappropriate and can become pathological, as in IBS-D $(11,24)$. Our data suggest that activation of $\mathrm{K}_{\mathrm{V}} 7$ channels in the ENS both inhibits electrogenic secretion and stimulates electroneutral absorption in the colon. To assess whether these two outcomes were correlated, the change in $\mathrm{M}-\mathrm{S} \mathrm{Na}{ }^{+}$flux was plotted against the change in $\mathrm{I}_{\mathrm{SC}}$ recorded from the same tissue. Tissues from all experiments in this project were used for analysis that incorporated normal distal or proximal colon, where fluxes were measured before and after flupirtine administration $(n=20)$. Simple linear regression and correlation analysis revealed a significant relationship $\left(p<0.0001 ; r^{2}=0.6051\right)$ between the magnitude of $\mathrm{I}_{\mathrm{SC}}$ inhibition and the magnitude in M-S flux enhancement (Figure 8). Such a strong correlation between these effects is presumably the result of varying degrees of basal ENS activity being inhibited by $\mathrm{K}_{\mathrm{V}} 7$ activation, leading to a proportional (but opposite) response with respect to $\mathrm{Cl}^{-}$secretion and $\mathrm{Na}^{+}$absorption because both processes are governed by ENS input. 


\section{DISCUSSION}

Here we provide evidence that a $\mathrm{K}_{\mathrm{V}} 7$ channel activator, flupirtine, both suppresses neurogenic $\mathrm{Cl}^{-}$secretion and enhances NHE-3-dependent $\mathrm{Na}^{+}$absorption simultaneously in the rat colon. In support of this claim are the following observations: 1) Flupirtine-sensitive $\mathrm{K}_{\mathrm{V}} 7.2$ channels were detected by immunofluorescence in submucosal ganglia, and ENS-driven $\mathrm{Cl}^{-}$ secretion (via veratridine) was suppressed by flupirtine, while the response to epithelium stimulation directly (via VIP) was unaffected. 2) Flupirtine inhibited $\mathrm{I}_{\mathrm{SC}}$ and $\mathrm{G}_{\mathrm{TE}}$, and increased both $\mathrm{M}-\mathrm{S}$ and $\mathrm{Net} \mathrm{Na}^{+}$fluxes across rat colonic tissues, but this effect was not observed in the presence of ENS blockade via tetrodotoxin. 3) S3226 and amiloride, but not HOE694, significantly inhibited M-S and Net $\mathrm{Na}^{+}$fluxes in the presence of flupirtine, while NHE-3 localization near the apical membranes of surface epithelial cells was apparently enhanced in flupirtine-treated colon compared to control. 4) Flupirtine did not alter $\mathrm{Na}^{+}$fluxes in "aldo" rat distal colon, which has significantly reduced NHE-3 expression/activity. 5) Flupirtine inhibited $\mathrm{I}_{\mathrm{SC}}$ and $\mathrm{G}_{\mathrm{TE}}$, and increased both M-S and $\mathrm{Net} \mathrm{Na}^{+}$fluxes in the proximal colon, but not in the ileum, suggesting a colonspecific effect.

Under normal conditions, the primary function of the colon is to desiccate fecal material via active absorption of $\mathrm{Na}^{+}, \mathrm{Cl}^{-}$and water. Efferent signals from the ENS can halt these absorptive processes and induce a secretory response to either normal (e.g., mechanical distension) $(53,54)$ or pathological stimuli (e.g., infection or stress) $(55,56)$. Often, epithelial secretion occurs in conjunction with increased motility of colonic smooth muscle in order to propagate material onward toward its eventual elimination (57). Secretomotor neurons of the ENS are thus frequently targeted in the treatment of diarrheal GI disorders, such as IBS-D. Alosetron (serotonin receptor antagonist) and loperamide (opioid receptor agonist) are two of the most commonly prescribed 
IBS-D treatments, both of which dampen ENS activity $(58,59)$. However, these drugs and others that modulate these ENS activity are associated with adverse side effects, such as nausea, abdominal pain, constipation and dependence (26). Novel pharmacotherapies that dampen ENSdriven symptoms may therefore potentially have great therapeutic potential. The results from this study demonstrate that $\mathrm{K}_{\mathrm{V}} 7$ channel activators may be of use in this context, as both inhibition of $\mathrm{Cl}^{-}$secretion and stimulation of $\mathrm{Na}^{+}$absorption favor water absorption and would likely combat diarrhea. Furthermore, if the effects observed here are truly specific to the colon (see Fig. 7), potential off-target effects of interfering with ENS function in the small intestine (i.e., related to normal digestive function) may possibly be avoided as well. In vivo studies will be required for a more complete assessment of efficacy.

The clinical use of $\mathrm{K}_{\mathrm{V}} 7$ channel activators, including flupirtine, has historically been centered around the treatment of chronic pain and epilepsy (21). These drugs are effective analgesics and anticonvulsants because of their suppressive effects on neuronal excitability. Opening of $\mathrm{K}_{\mathrm{V}} 7$ channels in the neuronal plasma membrane produces a hyperpolarizing $\mathrm{K}^{+}$current that stabilizes membrane potential and thus dampens the response to incoming stimuli (60). However, the exact mechanism of action in terms of their analgesic effects is not completely clear, and it may involve interactions with $\gamma$-amino butyric acid (GABA) receptors, $G$ protein-coupled inward rectifier $\mathrm{K}^{+}$(GIRK) channels, or possibly voltage-dependent $\mathrm{Mg}^{2+}$ block of NMDA receptors $(61,62,63)$. Whether direct or indirect, here we show for the first time that the suppressive effects of $\mathrm{K}_{\mathrm{V}} 7$ channel activators can be seen in the context of the ENS and its modulation of multiple ion transport pathways in the colon. Somewhat related to this, Peiris et. al. demonstrated that activation of $\mathrm{K}_{\mathrm{V}} 7$ channels in sensory afferents with retigabine - a structural analog of flupirtine - blunted the nociceptive response to noxious stimuli in mouse and human colon (20). 
The potential benefits of targeting neuronal $\mathrm{K}_{\mathrm{V}} 7$ channels in the treatment of GI disorders such as IBS-D may therefore be multifaceted. Generalized GI-related side effects (i.e., nausea, abdominal discomfort) are associated with flupirtine use in patients treated for chronic pain. However, to our knowledge, there has not been an analysis to determine whether patients with pre-existing GI conditions, such as IBS-D, experienced these side effects differentially. A thorough review of the clinical data surrounding the use of flupirtine in patients would be useful in this respect.

One of the major findings from this study is that specifically NHE-3-mediated $\mathrm{Na}^{+}$ absorption is enhanced by $\mathrm{K}_{\mathrm{V}} 7$ activation. Modulation of NHE-3 activity by secretagogues originating from ENS neurons has been thoroughly described (29). Cholinergic and VIP-ergic neurons constitute the two primary secretomotor classes within the submucosal plexus of the colon $(8,64)$. Both of the second messenger systems induced by the activation of their target receptors $\mathrm{Ca}^{2+}$ and cAMP, respectively - inhibit NHE-3 activity by triggering its rapid internalization from the apical membranes of surface colonocytes. Our observations that NHE-3 surface localization is enhanced by treatment with flupirtine, and that NHE-3-dependent $\mathrm{Na}^{+}$absorption is increased by flupirtine to a degree which is correlated to the inhibition of $\mathrm{Cl}^{-}$secretion, fits well into the existing model of $\mathrm{Na}^{+}$and $\mathrm{Cl}^{-}$transport modulation by ENS input. In spite of this, the experimental data presented here offer no definitive insight regarding the intracellular events underlying the observed effects on $\mathrm{Na}^{+}$or $\mathrm{Cl}^{-}$transport. Further studies will be required to assess the involvement of cholinergic or VIP-ergic (or other) pathways, as well as to resolve the details of NHE-3 trafficking and/or other possible means of regulation in response to flupirtine.

Flupirtine is generally considered to be non-selective for $\mathrm{K}_{\mathrm{V}} 7$ channels. One member of this family, $\mathrm{K}_{\mathrm{V}} 7.1$, has a well-defined role in the colonic epithelium itself $(65) . \mathrm{K}_{\mathrm{V}} 7.1$ is expressed in crypt cells and localizes to the basolateral membrane to support apical $\mathrm{Cl}^{-}$secretion by 
maintaining the electrochemical driving force for sustained $\mathrm{Cl}^{-}$efflux (66). In this study, as well as recent studies from our lab, there was no indication that flupirtine stimulates $\mathrm{Cl}^{-}$secretion by opening basolateral $K_{V} 7.1$ channels, despite the drug being "non-selective" for the family subtypes. Importantly, another non-selective $\mathrm{K}_{\mathrm{V}} 7$ channel activator, retigabine, does not act on the $\mathrm{K}_{\mathrm{V}} 7.1$ subtype in vitro (16). While analysis of the effects of flupirtine, specifically, on $\mathrm{K}_{\mathrm{V}} 7.1$ is lacking, there have been reports that the channel may also be insensitive to flupirtine (21). Indeed, flupirtine and retigabine are very closely related in both structure and function. We have also observed no effect from flupirtine on basal or stimulated $\mathrm{Cl}^{-}$secretion in $\mathrm{T} 84$ cells, which express $K_{V} 7.1$ channels. $K_{V} 7.1$ does exhibit significant homology to other $K_{V} 7$ family members (67). The reason for the apparent insensitivity to these non-selective activators are unknown but may be related to the association of $\mathrm{K}_{\mathrm{V}} 7.1$ with an auxiliary $\operatorname{minK}(K C N E)$ subunit in the colonic epithelium $(68,69)$, or may be related to secondary effects on GABA or NMDA receptors, as mentioned above. Nevertheless, flupirtine clearly modulates epithelial transport in an ENSdependent manner, as evidenced by the attenuating effect of pre-treatment with tetrodotoxin on the response to flupirtine, as well as the similarity in $\mathrm{I}_{\mathrm{SC}}, \mathrm{G}_{\mathrm{TE}}$ and $\mathrm{Na}^{+}$absorption properties among tissues treated with either of the two agents. The mechanistic details underlying $\mathrm{K}_{\mathrm{V}} 7$ activation of neuronal versus epithelial subtypes, as well as possible "off-target" inhibitory effects within ENS neurons, remain to be elucidated but should be the subject of future studies.

\section{CONCLUSIONS}

In summary, these data show that the $\mathrm{K}_{\mathrm{V}} 7$ channel activator, flupirtine, simultaneously inhibits $\mathrm{Cl}^{-}$secretion and stimulates NHE-3-dependent $\mathrm{Na}^{+}$absorption in the rat colon. Further, these effects are likely mediated by an ENS-dependent mechanism. Both of inhibition of $\mathrm{Cl}^{-}$ 
secretion and stimulation of $\mathrm{Na}^{+}$absorption may have significant bearing on water absorption in the colon. Therefore, the use of $\mathrm{K}_{\mathrm{V}} 7$-activating drugs for the treatment of ENS-associated diarrheal conditions - such as IBS-D - should be explored in the future.

*All authors have reviewed and approved this manuscript in its current form.

ACKNOWLEDGEMENTS: We thank Avinash Elangovan for his contributions in laboratory operations and animal care. This study was supported by the National Institute of Health NIDDK R01DK104791 and DK112085 grants to VMR

CONFLICTS OF INTEREST: None to disclose. 


\section{REFERENCES}

1. Sandle GI. Salt and water absorption in the human colon: A modern appraisal. Gut. 1998;43(2):294-299. doi:10.1136/gut.43.2.294

2. Binder HJ, Rawlins L. Electrolyte large transport mucosa across isolated intestinal. Am J Physiol. 1973;225(5):1232-1239.

3. Tumamian SG, Binder HJ. Regulation of active sodium and potassium transport in the distal colon of the rat. J Clin Invest. 1989;84(6):1924-1929.

4. Kunzelmann K, Mall M. Electrolyte transport in the mammalian colon: mechanisms and implications for disease. Physiol Rev. 2002;82(1):245-289. doi:10.1152/physrev.00026.2001

5. Ewe K. Intestinal transport in constipation and diarrhoea. Pharmacology. 1988;36 Suppl 1:73-84. doi:10.1159/000138424

6. Gershon MD. Nerves, reflexes, and the enteric nervous system: pathogenesis of the irritable bowel syndrome. J Clin Gastroenterol. 2005;39(5 Suppl 3):S184-93. doi:10.1097/01.mcg.0000156403.37240.30

7. Furness JB. The enteric nervous system and neurogastroenterology. Nat Rev Gastroenterol Hepatol. 2012;9(5):286-294. doi:10.1038/nrgastro.2012.32

8. Neunlist M, Reichelt D, Michel K, Pfannkuche H, Hoppe S, Schemann M. The Enteric Nervous System: Region and Target Specific Projections and Neurochemical Codes. Eur J Morphol. 1999;37(4):233-240. doi:10.1076/ejom.37.4.233.4720

9. Furness JB, Callaghan BP, Rivera LR, Cho H-J. The Enteric Nervous System and Gastrointestinal Innervation: Integrated Local and Central Control. In: Lyte M, Cryan JF, eds. Microbial Endocrinology: The Microbiota-Gut-Brain Axis in Health and Disease. New York, NY: Springer New York; 2014:39-71. doi:10.1007/978-1-4939-0897-4_3

10. Cooke HJ. Role of the "little brain" in the gut in water and electrolyte homeostasis 1 . FASEB J. 1989;3(2):127-138. doi:10.1096/fasebj.3.2.2464517

11. Camilleri M. Intestinal secretory mechanisms in irritable bowel syndrome-diarrhea. Clin Gastroenterol Hepatol Off Clin Pract J Am Gastroenterol Assoc. 2015;13(6):1051-1052. doi:10.1016/j.cgh.2014.07.020

12. Kuwahara A, Radowicz-Cooke HJ. Epithelial transport in guinea-pig proximal colon: influence of enteric neurones. J Physiol. 1988;395:271-284.

doi:10.1113/jphysiol.1988.sp016918 
13. Bridges BYRJ, Rack M, Rummel W, Schreiner J, Saar DH. Mucosal Plexus and Electrolyte Transport Across the Rat Colonic Mucosa. J Physiol. 1986;376:531-542. doi:10.1113/jphysiol.1986.sp016168

14. Nickerson AJ, Rottgen TS, Rajendran VM. Activation of KCNQ (K(V)7) K(+) channels in enteric neurons inhibits epithelial $\mathrm{Cl}(-)$ secretion in mouse distal colon. Am J Physiol Cell Physiol. April 2021. doi:10.1152/ajpcell.00536.2020

15. Wang HS, Pan Z, Shi W, et al. KCNQ2 and KCNQ3 potassium channel subunits: molecular correlates of the M-channel. Science. 1998;282(5395):1890-1893. doi:10.1126/science.282.5395.1890

16. Miceli F, Soldovieri MV, Martire M, Taglialatela M. Molecular pharmacology and therapeutic potential of neuronal Kv7-modulating drugs. Curr Opin Pharmacol. 2008;8(1):65-74. doi:10.1016/j.coph.2007.10.003

17. Brown DA, Passmore GM. Neural KCNQ (Kv7) channels. Br J Pharmacol. 2009;156(8):1185-1195. doi:10.1111/j.1476-5381.2009.00111.x

18. Shah MM, Migliore M, Valencia I, Cooper EC, Brown DA. Functional significance of axonal Kv7 channels in hippocampal pyramidal neurons. Proc Natl Acad Sci US A. 2008;105(22):7869-7874. doi:10.1073/pnas.0802805105

19. Watanabe H, Nagata E, Kosakai A, et al. Disruption of the epilepsy KCNQ2 gene results in neural hyperexcitability. J Neurochem. 2000;75(1):28-33. doi:10.1046/j.14714159.2000.0750028.x

20. Peiris M, Hockley JR, Reed DE, Smith ESJ, Bulmer DC, Blackshaw LA. Peripheral $\mathrm{K}(\mathrm{V}) 7$ channels regulate visceral sensory function in mouse and human colon. Mol Pain. 2017;13:1744806917709371. doi:10.1177/1744806917709371

21. Lawson K. Pharmacology and clinical applications of flupirtine: Current and future options. World J Pharmacol. 2019;3192(1):1-13.

22. Gunthorpe MJ, Large CH, Sankar R. The mechanism of action of retigabine (ezogabine), a first-in-class $\mathrm{K}+$ channel opener for the treatment of epilepsy. Epilepsia. 2012;53(3):412-424. doi:10.1111/j.1528-1167.2011.03365.x

23. Saha L. Irritable bowel syndrome: pathogenesis, diagnosis, treatment, and evidence-based medicine. World J Gastroenterol. 2014;20(22):6759-6773. doi:10.3748/wjg.v20.i22.6759

24. Mearin F, Lacy BE, Chang L, et al. Bowel Disorders. Gastroenterology. February 2016. doi:10.1053/j.gastro.2016.02.031

25. Buono JL, Mathur K, Averitt AJ, Andrae DA. Economic burden of inadequate symptom control among US commercially insured patients with irritable bowel syndrome with 
diarrhea. J Med Econ. 2017;20(4):353-362. doi:10.1080/13696998.2016.1269016

26. Cangemi DJ, Lacy BE. Management of irritable bowel syndrome with diarrhea: a review of nonpharmacological and pharmacological interventions. Therap Adv Gastroenterol. 2019;12:1756284819878950. doi:10.1177/1756284819878950

27. Gallo-Torres H, Brinker A, Avigan M. Alosetron: ischemic colitis and serious complications of constipation. Am J Gastroenterol. 2006;101(5):1080-1083. doi:10.1111/j.1572-0241.2006.00650.x

28. Page JG, Dirnberger GM. Treatment of the irritable bowel syndrome with Bentyl (dicyclomine hydrochloride). J Clin Gastroenterol. 1981;3(2):153-156. doi:10.1097/00004836-198106000-00009

29. Zachos NC, Tse M, Donowitz M. Molecular physiology of intestinal Na+/H+ exchange. Annu Rev Physiol. 2005;67:411-443. doi:10.1146/annurev.physiol.67.031103.153004

30. Martin RS, Jones WJ, Hayslett JP. Animal model to study the effect of adrenal hormones on epithelial function. Kidney Int. 1983;24(3):386-391. doi:10.1038/ki.1983.171

31. Singh SK, O’Hara B, Talukder JR, Rajendran VM. Aldosterone induces active K+ secretion by enhancing mucosal expression of Kcnn4c and Kcnmal channels in rat distal colon. Am J Physiol - Cell Physiol. 2012;302(9):1353-1360.

doi:10.1152/ajpcell.00216.2011

32. Amasheh S, Epple HJ, Mankertz J, et al. Differential Regulation of ENaC by Aldosterone in Rat Early and Late Distal Colon. Ann N Y Acad Sci. 2006;915(1):92-94. doi:https://doiorg.wvu.idm.oclc.org/10.1111/j.1749-6632.2000.tb05227.x

33. Halevy J, Budinger ME, Hayslett JP, Binder HJ. Role of Aldosterone in the Regulation of Sodium and Chloride Transport in the Distal Colon of Sodium-Depleted Rats. Gastroenterology. 1986;91(5):1227-1233. doi:https://doi.org/10.1016/S00165085(86)80021-X

34. Escoubet B, Coureau C, Bonvalet JP, Farman N. Noncoordinate regulation of epithelial na channel and Na pump subunit mRNAs in kidney and colon by aldosterone. Am J Physiol Cell Physiol. 1997;272(5 41-5). doi:10.1152/ajpcell.1997.272.5.c1482

35. Greig ER, Baker EH, Mathialahan T, Boot-Handford RP, Sandle GI. Segmental variability of ENaC subunit expression in rat colon during dietary sodium depletion. Pflugers Arch Eur J Physiol. 2002;444(4):476-483. doi:10.1007/s00424-002-0828-7

36. Lomax B, Mcnicholas M, Lombes M, Sandle I, Mcnicholas M. Aldosterone-induced apical $\mathrm{Na}+$ and $\mathrm{K}+$ conductances are located primarily in surface cells in rat distal colon. 1994;29:G71-G82. 
37. Saint-Criq V, Rapetti-Mauss R, Yusef YR, Harvey BJ. Estrogen regulation of epithelial ion transport: Implications in health and disease. Steroids. 2012;77(10):918-923. doi:10.1016/j.steroids.2012.02.017

38. O’Mahony F, Thomas W, Harvey BJ. Novel female sex-dependent actions of oestrogen in the intestine. J Physiol. 2009;587(Pt 21):5039-5044. doi:10.1113/jphysiol.2009.177972

39. Choijookhuu N, Sato Y, Nishino T, Endo D, Hishikawa Y, Koji T. Estrogen-dependent regulation of sodium/hydrogen exchanger-3 (NHE3) expression via estrogen receptor $\beta$ in proximal colon of pregnant mice. Histochem Cell Biol. 2012;137(5):575-587. doi:10.1007/s00418-012-0935-2

40. Clarke LL. A guide to Ussing chamber studies of mouse intestine. AJP Gastrointest Liver Physiol. 2009;296(6):G1151-G1166. doi:10.1152/ajpgi.90649.2008

41. Liu C, Lin C, Whitaker DT, et al. Prickle1 is expressed in distinct cell populations of the central nervous system and contributes to neuronal morphogenesis. Hum Mol Genet. 2013;22(11):2234-2246. doi:10.1093/hmg/ddt075

42. Bane V, Lehane M, Dikshit M, O’Riordan A, Furey A. Tetrodotoxin: chemistry, toxicity, source, distribution and detection. Toxins (Basel). 2014;6(2):693-755.

doi:10.3390/toxins6020693

43. Ikuma M, Kashgarian M, Binder HJ, Rajendran VM. Differential regulation of NHE isoforms by sodium depletion in proximal and distal segments of rat colon. Am J Physiol Gastrointest Liver Physiol. 1999;276(2 39-2):539-549. doi:10.1152/ajpgi.1999.276.2.g539

44. Subramanya SB, Rajendran VM, Srinivasan P, Nanda Kumar NS, Ramakrishna BS, Binder HJ. Differential regulation of cholera toxin-inhibited Na-H exchange isoforms by butyrate in rat ileum. Am J Physiol Gastrointest Liver Physiol. 2007;293(4):G857-63. doi:10.1152/ajpgi.00462.2006

45. Rajendran VM, Nanda Kumar NS, Tse CM, Binder HJ. Na-H Exchanger Isoform-2 (NHE2) Mediates Butyrate-dependent Na+ Absorption in Dextran Sulfate Sodium (DSS)induced Colitis. J Biol Chem. 2015;290(42):25487-25496. doi:10.1074/jbc.M115.654277

46. Pasham V, Pathare G, Fajol A, et al. OSR1-sensitive small intestinal $\mathrm{Na}+$ transport. Am J Physiol Gastrointest Liver Physiol. 2012;303(11):G1212-9. doi:10.1152/ajpgi.00367.2011

47. Foster ES, Budinger ME, Hayslett JP, Binder HJ. Ion transport in proximal colon of the rat. Sodium depletion stimulates neutral sodium chloride absorption. J Clin Invest. 1986;77(1):228-235. doi:10.1172/JCI112281

48. Donowitz M, Mohan S, Zhu CX, et al. NHE3 regulatory complexes. J Exp Biol. 2009;212(Pt 11):1638-1646. doi:10.1242/jeb.028605 
49. Schnizler M, Mastroberardino L, Reifarth F, Weber WM, Verrey F, Clauss W. cAMP sensitivity conferred to the epithelial $\mathrm{Na}+$ channel by alpha-subunit cloned from guineapig colon. Pflugers Arch. 2000;439(5):579-587. doi:10.1007/s004249900213

50. Segal A, Cucu D, Van Driessche W, Weber WM. Rat ENaC expressed in Xenopus laevis oocytes is activated by cAMP and blocked by $\mathrm{Ni}(2+)$. FEBS Lett. 2002;515(1-3):177-183. doi:10.1016/s0014-5793(02)02464-X

51. Rao US, Baker JM, Pluznick JL, Balachandran P. Role of intracellular Ca2+ in the expression of the amiloride-sensitive epithelial sodium channel. Cell Calcium. 2004;35(1):21-28. doi:10.1016/s0143-4160(03)00157-x

52. Inagaki A, Yamaguchi S, Ishikawa T. Amiloride-sensitive epithelial $\mathrm{Na}+$ channel currents in surface cells of rat rectal colon. Am J Physiol Cell Physiol. 2004;286(2):C380-90. doi:10.1152/ajpcell.00373.2003

53. Schulzke JD, Pfaffenbach S, Fromm A, Epple HJ, Troeger H, Fromm M. Prostaglandin I(2) sensory input into the enteric nervous system during distension-induced colonic chloride secretion in rat colon. Acta Physiol (Oxf). 2010;199(3):305-316.

doi:10.1111/j.1748-1716.2010.02096.x

54. Diener M, Rummel W. Distension-induced secretion in the rat colon: mediation by prostaglandins and submucosal neurons. Eur J Pharmacol. 1990;178(1):47-57. doi:10.1016/0014-2999(90)94792-v

55. Lundgren O, Peregrin AT, Persson K, Kordasti S, Uhnoo I, Svensson L. Role of the enteric nervous system in the fluid and electrolyte secretion of rotavirus diarrhea. Science. 2000;287(5452):491-495. doi:10.1126/science.287.5452.491

56. Million M, Larauche M. Stress, sex, and the enteric nervous system. Neurogastroenterol Motil Off J Eur Gastrointest Motil Soc. 2016;28(9):1283-1289. doi:10.1111/nmo.12937

57. Spencer NJ, Hu H. Enteric nervous system: sensory transduction, neural circuits and gastrointestinal motility. Nat Rev Gastroenterol Hepatol. 2020;17(6):338-351. doi:10.1038/s41575-020-0271-2

58. Houghton LA, Foster JM, Whorwell PJ. Alosetron, a 5-HT3 receptor antagonist, delays colonic transit in patients with irritable bowel syndrome and healthy volunteers. Aliment Pharmacol Ther. 2000;14(6):775-782. doi:10.1046/j.1365-2036.2000.00762.x

59. De Luca A, Coupar IM. Difenoxin and loperamide: studies on possible mechanisms of intestinal antisecretory action. Naunyn Schmiedebergs Arch Pharmacol. 1993;347(2):231-237. doi:10.1007/BF00169273

60. Peters HC, Hu H, Pongs O, Storm JF, Isbrandt D. Conditional transgenic suppression of $\mathrm{M}$ channels in mouse brain reveals functions in neuronal excitability, resonance and 
behavior. Nat Neurosci. 2005;8(1):51-60. doi:10.1038/nn1375

61. Klinger F, Bajric M, Salzer I, et al. $\delta$ Subunit-containing GABAA receptors are preferred targets for the centrally acting analgesic flupirtine. Br J Pharmacol. 2015;172(20):49464958. doi: $10.1111 / \mathrm{bph} .13262$

62. Jakob R, Krieglstein J. Influence of flupirtine on a G-protein coupled inwardly rectifying potassium current in hippocampal neurones. Br J Pharmacol. 1997;122(7):1333-1338. doi:10.1038/sj.bjp.0701519

63. Kornhuber J, Bleich S, Wiltfang J, Maler M, Parsons CG. Flupirtine shows functional NMDA receptor antagonism by enhancing $\mathrm{Mg} 2+$ block via activation of voltage independent potassium channels. J Neural Transm. 1999;106(9-10):857-867. doi: $10.1007 / \mathrm{s} 007020050206$

64. Foong JPP, Tough IR, Cox HM, Bornstein JC. Properties of cholinergic and noncholinergic submucosal neurons along the mouse colon. J Physiol. 2014;592(4):777-793. doi:10.1113/jphysiol.2013.265686

65. MacVinish LJ, Hickman ME, Mufti DA, Durrington HJ, Cuthbert AW. Importance of basolateral $\mathrm{K}+$ conductance in maintaining $\mathrm{Cl}$ - secretion in murine nasal and colonic epithelia. J Physiol. 1998;510 ( Pt 1(Pt 1):237-247. doi:10.1111/j.1469-7793.1998.237bz.x

66. Kunzelmann K, Hübner M, Schreiber R, et al. Cloning and function of the rat colonic epithelial K+ channel KVLQT1. J Membr Biol. 2001;179(2):155-164. doi: $10.1007 / \mathrm{s} 002320010045$

67. Gribkoff VK. The therapeutic potential of neuronal KCNQ channel modulators. Expert Opin Ther Targets. 2003;7(6):737-748. doi:10.1517/14728222.7.6.737

68. Barro-Soria R, Ramentol R, Liin SI, Perez ME, Kass RS, Larsson HP. KCNE1 and KCNE3 modulate KCNQ1 channels by affecting different gating transitions. Proc Natl Acad Sci U S A. 2017;114(35):E7367-E7376. doi:10.1073/pnas.1710335114

69. Liao T, Wang L, Halm ST, Lu L, Fyffe REW, Halm DR. K+ channel KVLQT1 located in the basolateral membrane of distal colonic epithelium is not essential for activating Clsecretion. Am J Physiol Cell Physiol. 2005;289(3):C564-75.

doi:10.1152/ajpcell.00561.2004 


\section{TABLES}

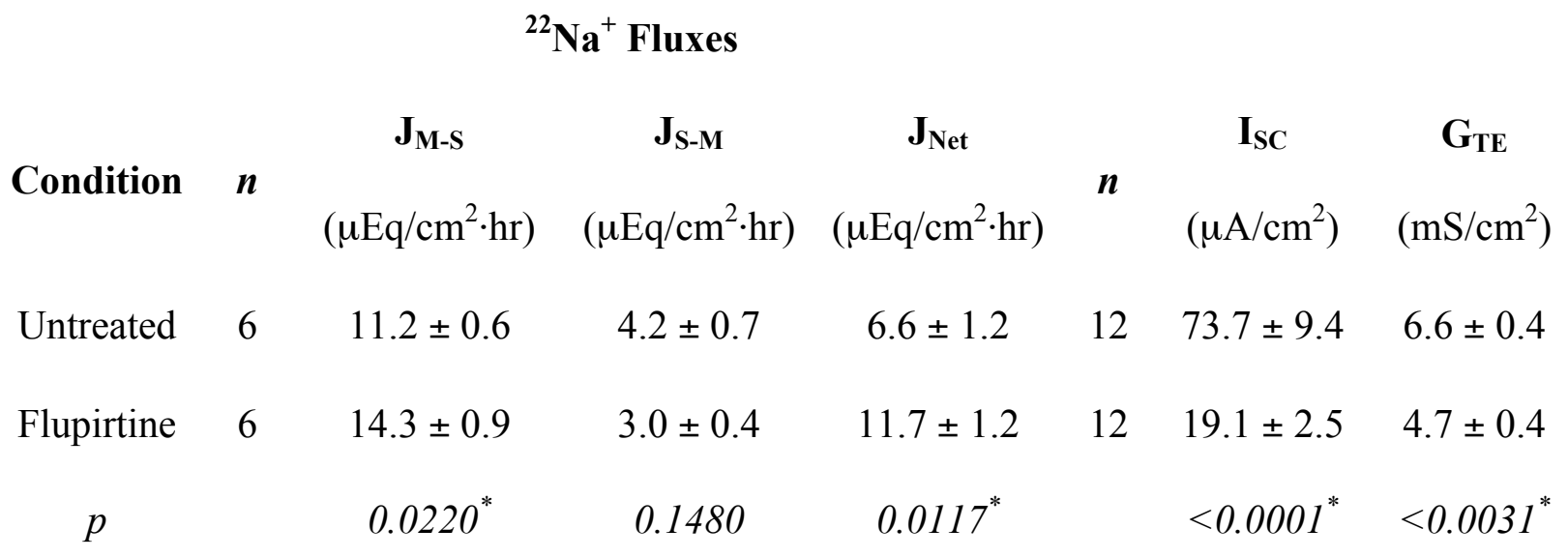

Table 1 - Effect of flupirtine on unidirectional ${ }^{22} \mathrm{Na}^{+}$fluxes in rat distal colon. Unidirectional

${ }^{22} \mathrm{Na}^{+}$fluxes were measured across seromuscular-stripped rat distal colon under voltage-clamped conditions in the presence and absence of $100 \mu \mathrm{M}$ serosal flupirtine. Mucosal-to-serosal (M-S) and serosal-to-mucosal (S-M) fluxes were measured over a 30-minute period. Net fluxes were calculated by subtracting S-M from M-S fluxes using tissues pairs matched by conductance, as described in Methods. Flux values are given as mean \pm SEM from 6 tissue pairs. $\mathrm{I}_{\mathrm{SC}}$ and $\mathrm{G}_{\mathrm{TE}}$ values represent the averages recorded over the 30 -minute flux period and are given as mean \pm SEM from 12 tissues (6 tissues each for M-S and S-M fluxes). $p$ values are given for each treatment group/parameter. ${ }^{*}$ indicates significance compared to control (untreated), as determined by unpaired Student's t-test. 


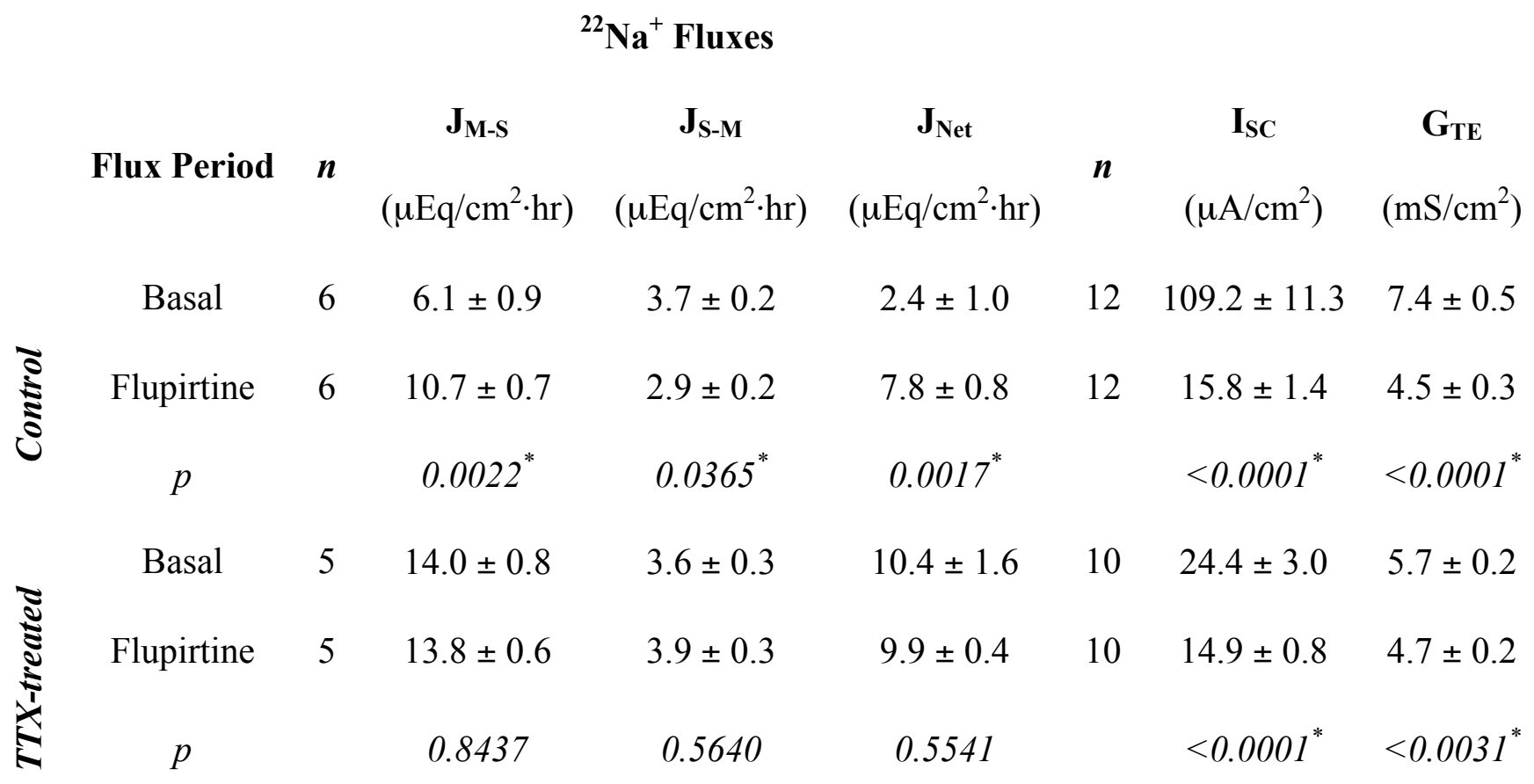

Table 2 - Effect of tetrodotoxin on flupirtine-stimulated ${ }^{22} \mathrm{Na}^{+}$fluxes in rat distal colon. Unidirectional ${ }^{22} \mathrm{Na}^{+}$fluxes were measured across seromuscular-stripped rat distal colon under voltage-clamped conditions before (Basal) and after the addition of $100 \mu \mathrm{M}$ flupirtine (Flupirtine) to the serosal chamber bath in the absence (Control) and presence of tetrodotoxin (TTX-treated). Flux values are given mean \pm SEM from 6 tissue pairs from control and 5 tissue pairs from the tetrodotoxin-treated group. $\mathrm{I}_{\mathrm{SC}}$ and $\mathrm{G}_{\mathrm{TE}}$ values represent the averages recorded over final 5 minutes of each 30-minute flux period and are given as mean \pm SEM from 12 control and 10 tetrodotoxintreated tissues. $p$ values are given for each treatment group/parameter. ${ }^{*}$ indicates significance when compared to the basal flux period, as determined by unpaired Student's t-test. 


\begin{tabular}{|c|c|c|c|c|c|c|c|}
\hline \multirow[b]{3}{*}{ Condition } & \multicolumn{4}{|c|}{${ }^{22} \mathrm{Na}^{+}$Fluxes } & \multirow[b]{3}{*}{$n$} & \multirow{3}{*}{$\begin{array}{c}\mathbf{I}_{\mathbf{S C}} \\
\left(\mu \mathrm{A} / \mathrm{cm}^{2}\right)\end{array}$} & \multirow{3}{*}{$\begin{array}{c}\mathbf{G}_{\mathbf{T E}} \\
\left(\mathrm{mS} / \mathrm{cm}^{2}\right)\end{array}$} \\
\hline & & $\mathbf{J}_{\mathbf{M}-\mathbf{S}}$ & $\mathbf{J}_{\mathrm{S}-\mathbf{M}}$ & $\mathbf{J}_{\text {Net }}$ & & & \\
\hline & $n$ & $\left(\mu \mathrm{Eq} / \mathrm{cm}^{2} \cdot \mathrm{hr}\right)$ & $\left(\mu \mathrm{Eq} / \mathrm{cm}^{2} \cdot \mathrm{hr}\right)$ & $\left(\mu \mathrm{Eq} / \mathrm{cm}^{2} \cdot \mathrm{hr}\right)$ & & & \\
\hline Flupirtine & 5 & $14.1 \pm 0.3$ & $3.2 \pm 0.2$ & $10.5 \pm 0.4$ & 10 & $19.6 \pm 2.0$ & $5.3 \pm 0.5$ \\
\hline HOE694 & 5 & $13.0 \pm 0.7$ & $3.0 \pm 0.2$ & $10.0 \pm 0.5$ & 10 & $17.7 \pm 1.8$ & $5.6 \pm 0.5$ \\
\hline S3226 & 5 & $6.5 \pm 0.4^{*, \#}$ & $2.2 \pm 0.2^{*}, \#$ & $4.2 \pm 0.3^{*}, \#$ & 10 & $12.8 \pm 0.8^{*}$ & $4.0 \pm 0.1^{*, \#}$ \\
\hline Amiloride & 5 & $4.3 \pm 0.5^{*, \#, £}$ & $2.4 \pm 0.1^{*}$ & $1.9 \pm 0.5^{*, \#, \Sigma}$ & 10 & $13.3 \pm 0.9^{*}$ & $4.5 \pm 0.1$ \\
\hline
\end{tabular}

\section{Table 3 - Effect of HOE694, S3226 and amiloride on unidirectional ${ }^{22} \mathrm{Na}^{+}$fluxes in flupirtine-}

treated rat distal colon. Unidirectional ${ }^{22} \mathrm{Na}^{+}$fluxes were measured across seromuscular-stripped rat distal colon under voltage-clamped conditions in the presence of $100 \mu \mathrm{M}$ serosal flupirtine alone, or flupirtine plus $50 \mu \mathrm{M}$ mucosal HOE694, $10 \mu \mathrm{M}$ mucosal S3226 or $1 \mathrm{mM}$ mucosal amiloride. Flux values are given as mean \pm SEM from 5 tissue pairs for all treatment groups. $\mathrm{I}_{\mathrm{SC}}$ and $\mathrm{G}_{\mathrm{TE}}$ values were averaged from 10 tissues ( 5 tissue pairs) over the 30 -minute flux period for each treatment group and are given as mean \pm SEM. ${ }^{*} p<0.01$ versus flupirtine alone, ${ }^{\#} p<0.05$ versus flupirtine plus HOE694, ${ }^{£} p<0.05$ versus flupirtine plus S3226, as determined by one-way ANOVA with Tukey's post-hoc. 


\begin{tabular}{|c|c|c|c|c|c|c|c|}
\hline & & & $\mathrm{Na}^{+}$Fluxes & & & & \\
\hline & & $\mathbf{J}_{\mathbf{M}-\mathbf{S}}$ & $\mathbf{J}_{\mathbf{S - M}}$ & $\mathbf{J}_{\text {Net }}$ & & $\mathbf{I}_{\mathbf{S C}}$ & $\mathbf{G}_{\mathrm{TE}}$ \\
\hline Condition & $n$ & $\left(\mu \mathrm{Eq} / \mathrm{cm}^{2} \cdot \mathrm{hr}\right)$ & $\left(\mu \mathrm{Eq} / \mathrm{cm}^{2} \cdot \mathrm{hr}\right)$ & $\left(\mu \mathrm{Eq} / \mathrm{cm}^{2} \cdot \mathrm{hr}\right)$ & $n$ & $\left(\mu \mathrm{A} / \mathrm{cm}^{2}\right)$ & $\left(\mathrm{mS} / \mathrm{cm}^{2}\right)$ \\
\hline Basal & 5 & $13.9 \pm 0.7$ & $2.4 \pm 0.2$ & $11.4 \pm 0.7$ & 10 & $297.8 \pm 7.2$ & $11.8 \pm 0.7$ \\
\hline Flupirtine & 5 & $12.3 \pm 0.8$ & $1.6 \pm 0.2^{*}$ & $10.7 \pm 0.8$ & 10 & $195.5 \pm 11.8^{*}$ & $7.4 \pm 0.3^{*}$ \\
\hline Amiloride & 5 & $3.7 \pm 0.3^{*, \#}$ & $1.7 \pm 0.1^{*}$ & $2.0 \pm 0.3^{*, \#}$ & 10 & $-27.9 \pm 2.5^{*}$ & $5.0 \pm 0.2^{*, \#}$ \\
\hline
\end{tabular}

Table 4 - Effect of flupirtine and amiloride on unidirectional ${ }^{22} \mathrm{Na}^{+}$fluxes in "aldo" rat distal colon. Unidirectional ${ }^{22} \mathrm{Na}^{+}$fluxes were measured across seromuscular-stripped "aldo" rat distal colon under voltage-clamped conditions for three sequential flux periods: under basal conditions, in the presence of $100 \mu \mathrm{M}$ serosal flupirtine, and flupirtine plus $10 \mu \mathrm{M}$ mucosal amiloride. Flux values are given as mean \pm SEM from 5 tissue pairs for all treatment groups. $\mathrm{I}_{\mathrm{SC}}$ and $\mathrm{G}_{\mathrm{TE}}$ values were averaged from the 10 tissues ( 5 tissue pairs) over the final 5 minutes of each 30 -minute flux period for each treatment group and are also given as mean \pm SEM. ${ }^{*} p<0.01$ versus basal flux period, ${ }^{\#} p<0.05$ versus flupirtine, as determined by one-way ANOVA with Tukey's post-hoc. 
FIGURES AND LEGENDS

A

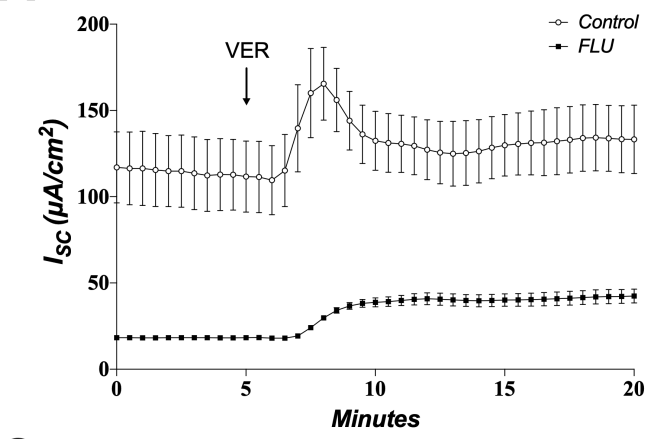

C

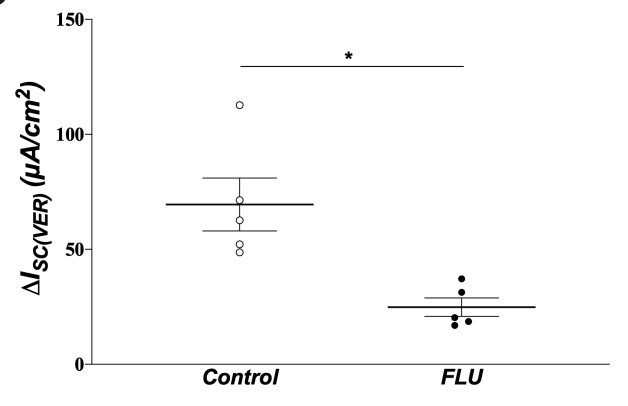

B

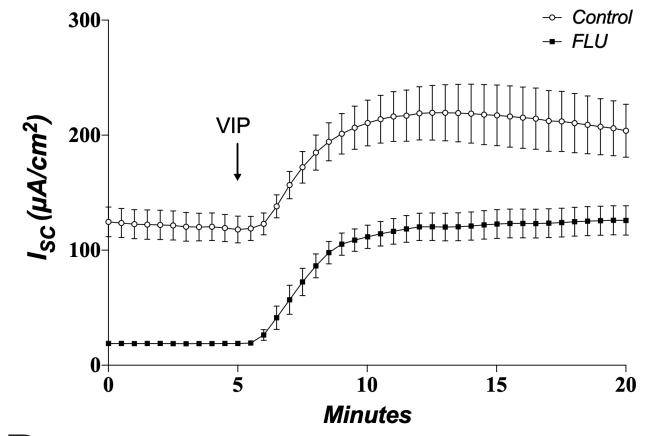

D

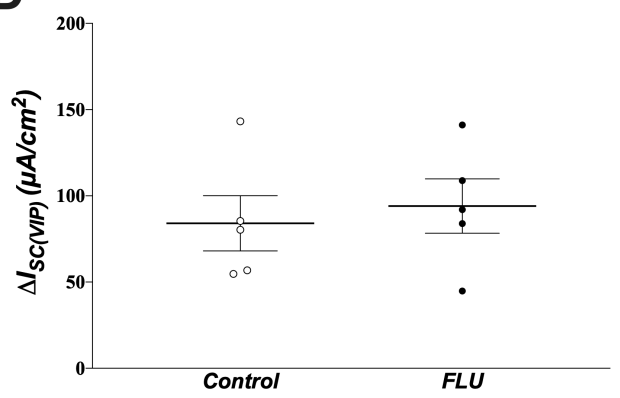

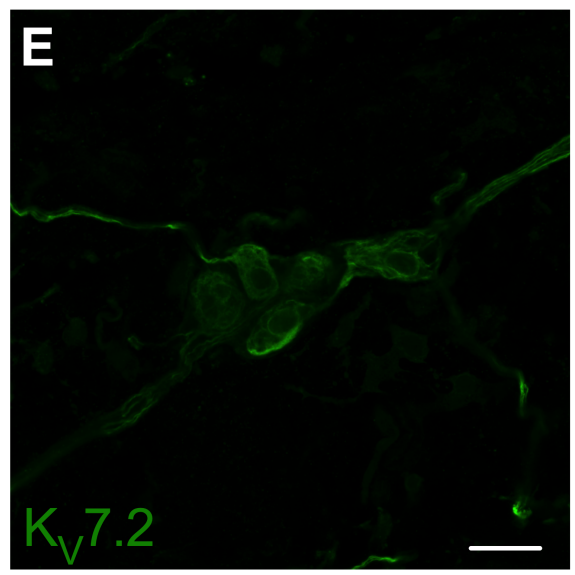
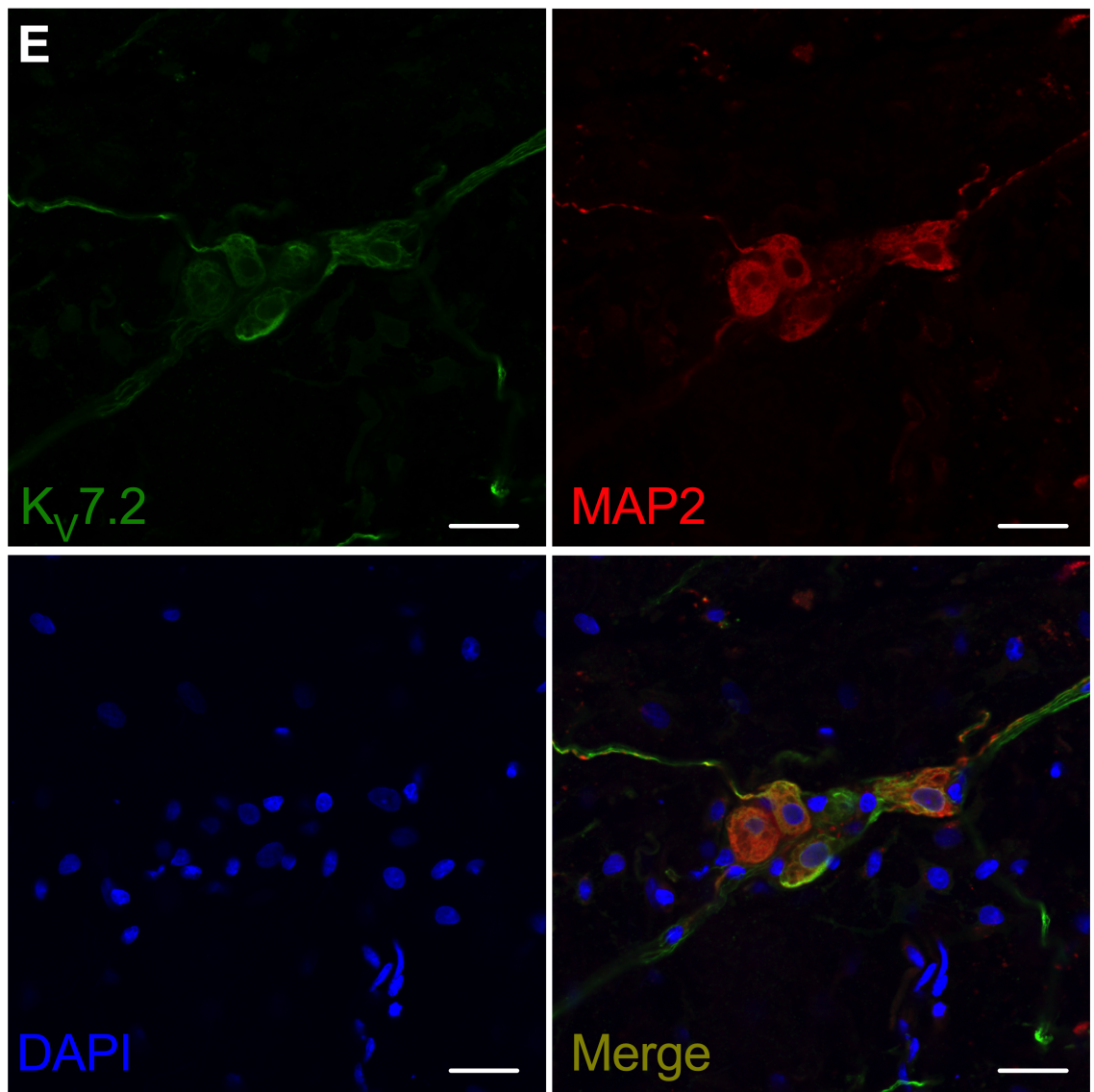
Figure 1 - Flupirtine inhibits neurogenic $\mathrm{Cl}^{-}$secretion in rat distal colon. $A$ - $B$ : Short-circuit current $\left(\mathrm{I}_{\mathrm{SC}}\right)$ recordings from control (open circles) and $100 \mu \mathrm{M}$ flupirtine-treated (closed circles) rat distal colon in response to $10 \mu \mathrm{M}$ veratridine (VER) $(A)$ or $100 \mathrm{nM}$ vasoactive intestinal polypeptide (VIP) (B). All drugs were added to the serosal chamber bath. $C$ : Group data showing the change in $\mathrm{I}_{\mathrm{SC}}$ following veratridine administration in control and flupirtine-treated tissues. $D$ : Group data showing the change in $\mathrm{I}_{\mathrm{SC}}$ following VIP administration in control and flupirtinetreated tissues. Lines and error bars represent mean \pm SEM. $n=5$ tissues obtained from at least 3 separate animals for all groups; ${ }^{*} p<0.05$, as determined by unpaired Student's t-test. $E$ : Immunofluorescent labeling of flupirtine-sensitive $\mathrm{K}_{\mathrm{V}} 7.2$ channels (green) in MAP2 ${ }^{+}$cells (red) from a submucosal ganglion of rat distal colon. Nuclei are labeled with DAPI (blue). Images were captured at 20x magnification. Scale bar $=20$ microns.
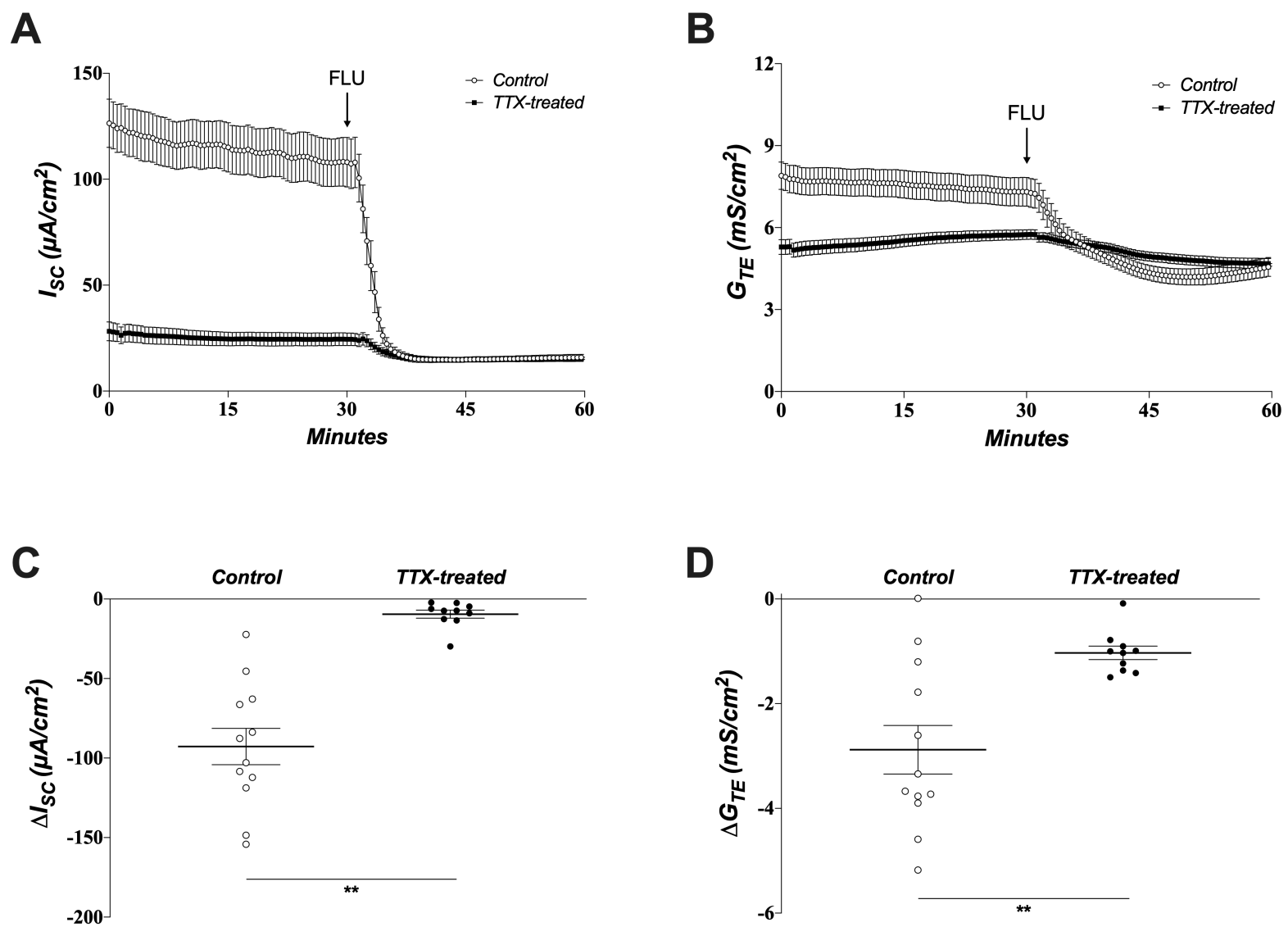
Figure 2 - Flupirtine-induced $\mathbf{I}_{\mathrm{SC}}$ and $\mathbf{G}_{\mathrm{TE}}$ inhibition is attenuated by tetrodotoxin. $A-B$ : $\mathrm{I}_{\mathrm{SC}}$ and $\mathrm{G}_{\mathrm{TE}}$ recordings from control (open circles) and $1 \mu \mathrm{M}$ tetrodotoxin $(T T X)$-treated (closed circles) seromuscular-stripped rat distal colon in response to $100 \mu \mathrm{M}$ flupirtine (FLU). All drugs were added to the serosal chamber bath. $C$ : Group data showing the change in $\mathrm{I}_{\mathrm{SC}}$ following flupirtine administration in control and tetrodotoxin-treated tissues. $D$ : Group data showing the change in $\mathrm{G}_{\mathrm{TE}}$ following flupirtine administration in control and tetrodotoxin-treated tissues. Lines and error bars represent mean \pm SEM. $n=12$ for control and $n=10$ for tetrodotoxin-treated tissues, obtained from 6 and 5 separate animals, respectively; ${ }^{* *} p<0.01$, as determined by unpaired Student's t-test. ${ }^{22} \mathrm{Na}^{+}$fluxes were also performed during these experiments and are summarized in Table 2. 
A Sequential flux periods (from Table 2)

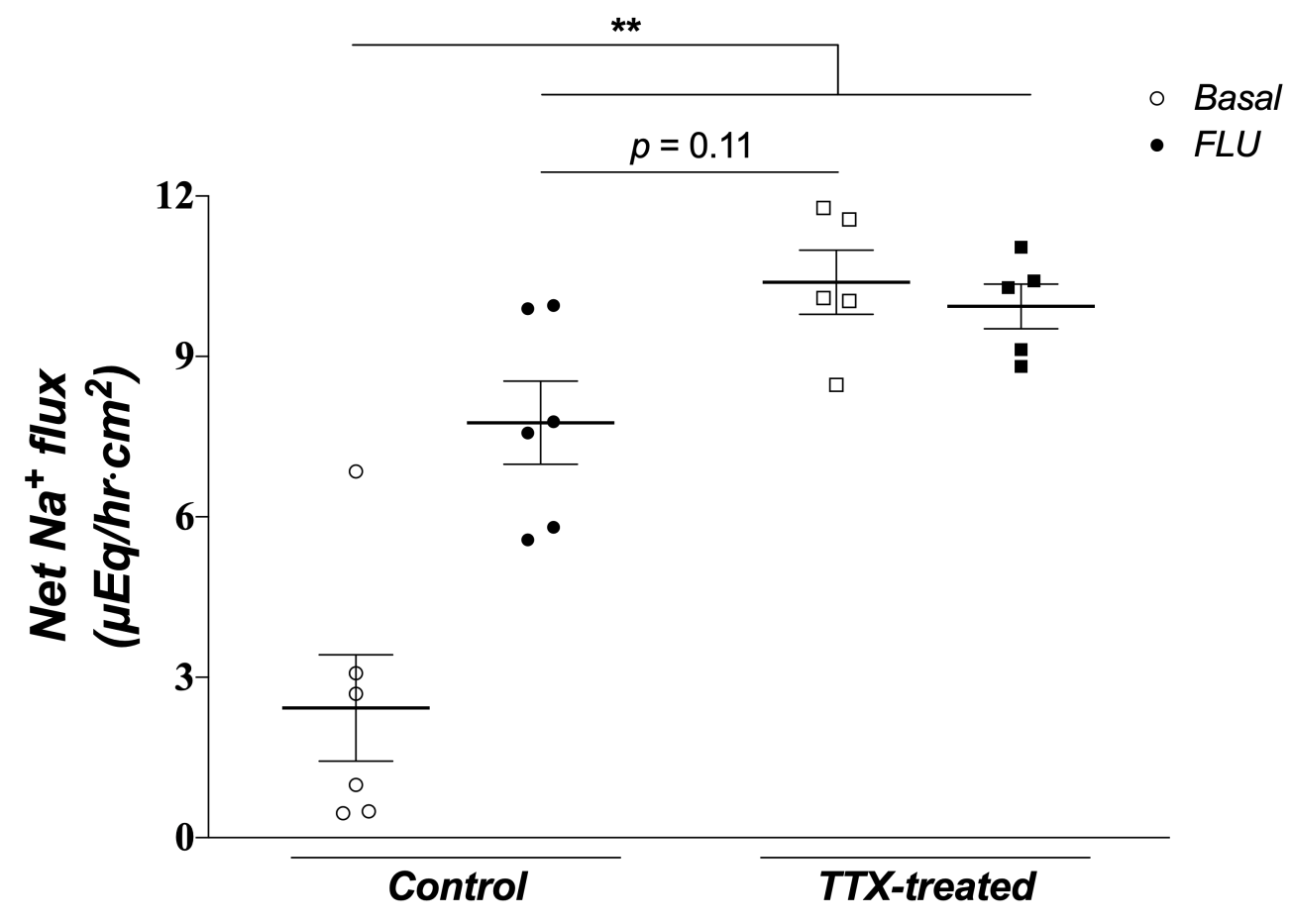

B Time-matched flux periods (from Tables 1 and 2)

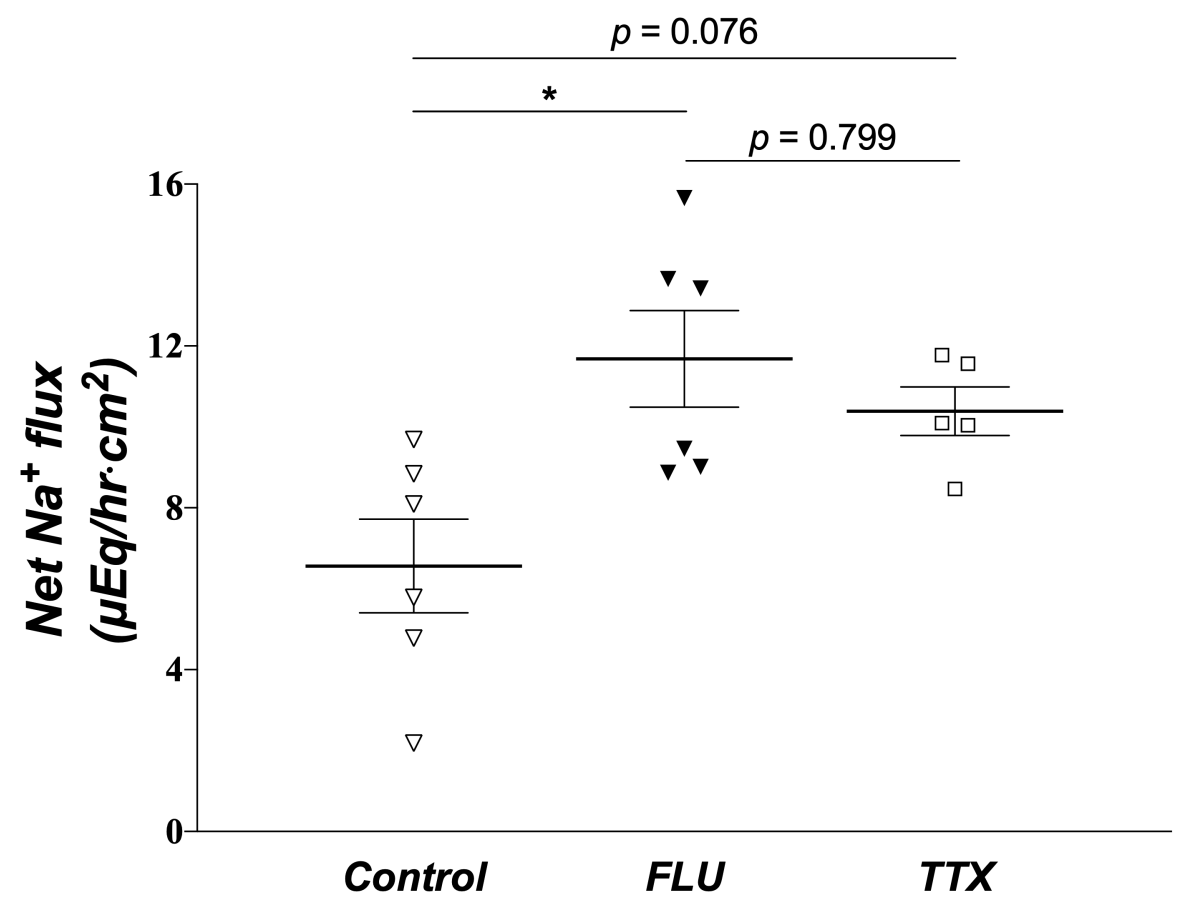


Figure 3 - Flupirtine enhances $\mathrm{Na}^{+}$absorption via a tetrodotoxin-sensitive mechanism. $A$ : Net $\mathrm{Na}^{+}$fluxes before (open circles/squares) and after (closed circles/squares) addition of $100 \mu \mathrm{M}$ serosal flupirtine in control and $1 \mu \mathrm{M}$ tetrodotoxin $(T T X)$-treated seromuscular-stripped rat distal colon. Values in panel $A$ are derived from Table 2. $B$ : $\mathrm{Net}^{+}{ }^{+}$fluxes in time-matched tissues that were untreated (Control, open triangles), treated with $100 \mu \mathrm{M}$ serosal flupirtine (FLU; closed triangles) or treated with $1 \mu \mathrm{M}$ serosal tetrodotoxin (TTX; open squares). Values in panel $B$ are derived from Tables 1 and 2. Lines and error bars represent mean \pm SEM from 6 (control) or 5 (tetrodotoxin-treated) tissue pairs obtained from 6 and 5 separate animals, respectively. ${ }^{*} p<0.05$, ** $p<0.01$, as determined by one-way ANOVA with Tukey’s post-hoc.

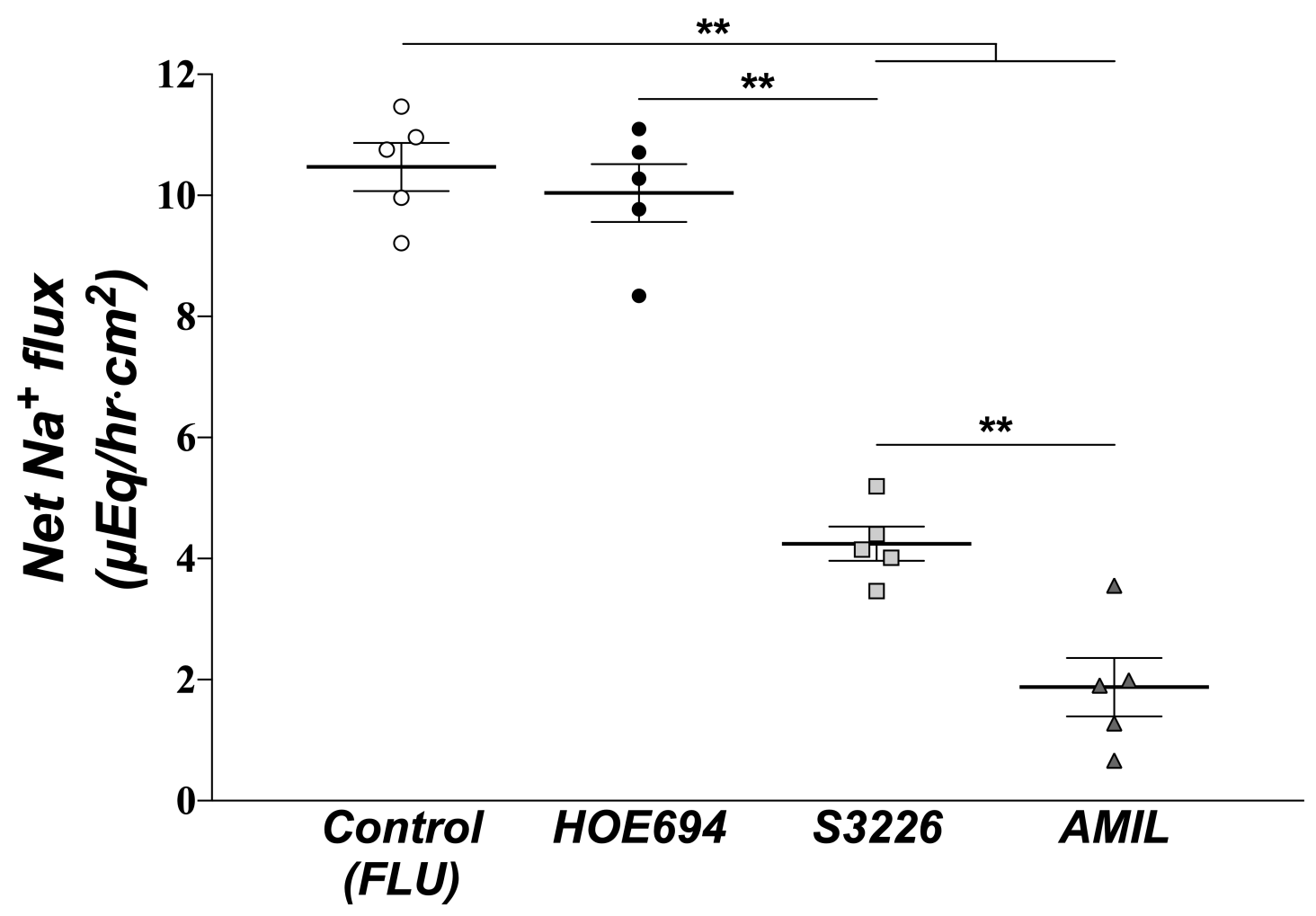

Figure 4 - Flupirtine-stimulated $\mathrm{Na}^{+}$absorption is primarily mediated by $\mathrm{Na}^{+} / \mathrm{H}^{+}$exchanger isoform 3 (NHE-3). Net $\mathrm{Na}^{+}$fluxes in the presence of $100 \mu \mathrm{M}$ serosal flupirtine alone (open circles), or flupirtine plus $50 \mu \mathrm{M}$ mucosal HOE694 (NHE-2-selective inhibitor; closed circles), 10 
$\mu \mathrm{M}$ mucosal S3226 (NHE-3-selective inhibitor; light gray squares) or $1 \mathrm{mM}$ mucosal amiloride (AMIL, non-selective NHE/ENaC inhibitor; dark gray triangles). Lines and error bars represent mean \pm SEM from 5 tissue pairs, obtained from 5 separate animals, for all groups. ${ }^{* *} p<0.01$, as determined by one-way ANOVA with Tukey's post-hoc.

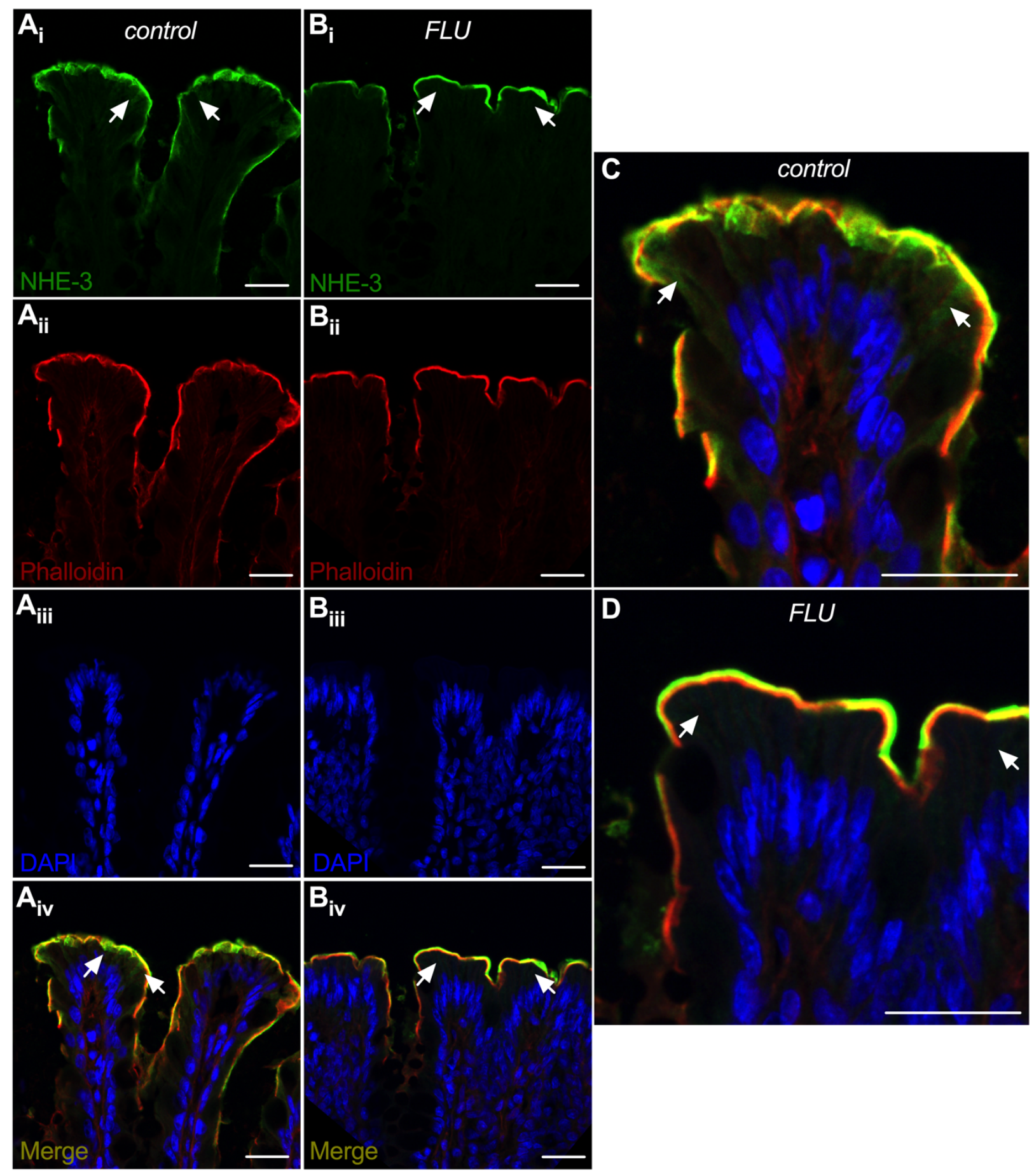


Figure 5 - Effect of flupirtine on surface localization of NHE-3 in rat distal colon. Seromuscular-stripped rat distal colon, incubated for 10 minutes in the absence (control, row $A$ ) or presence of flupirtine (FLU, row B), were labeled for NHE-3 (green) and filamentous actin (phalloidin, red). Nuclei are labeled with DAPI (blue). Zoom of control $(C)$ and flupirtine-treated (D) colon is also shown below. Arrows indicate the presence of sub-apical NHE-3 proteins, which are more prominent in control versus flupirtine-treated rat distal colon. Images were captured at 20x magnification. Scale bar $=25$ microns. 
A

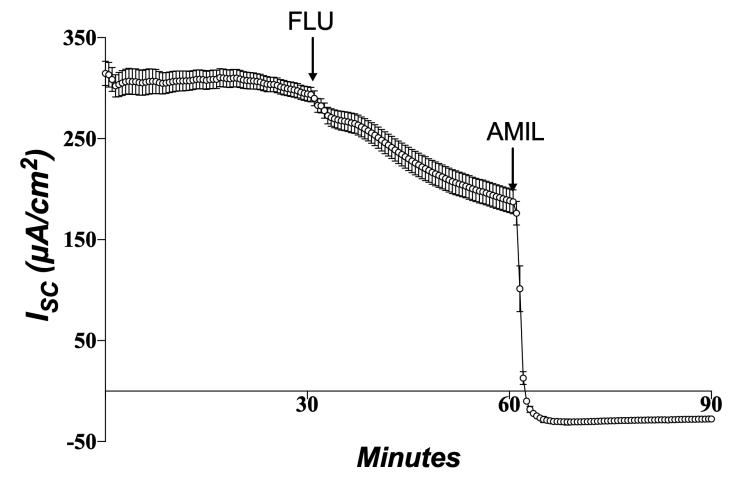

B

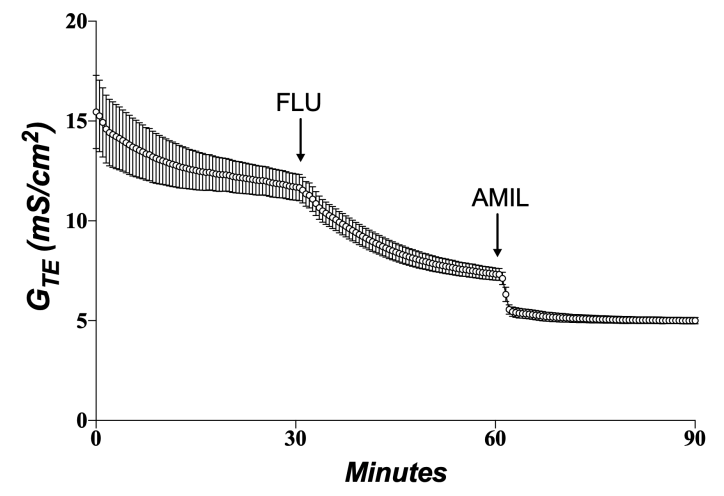

C

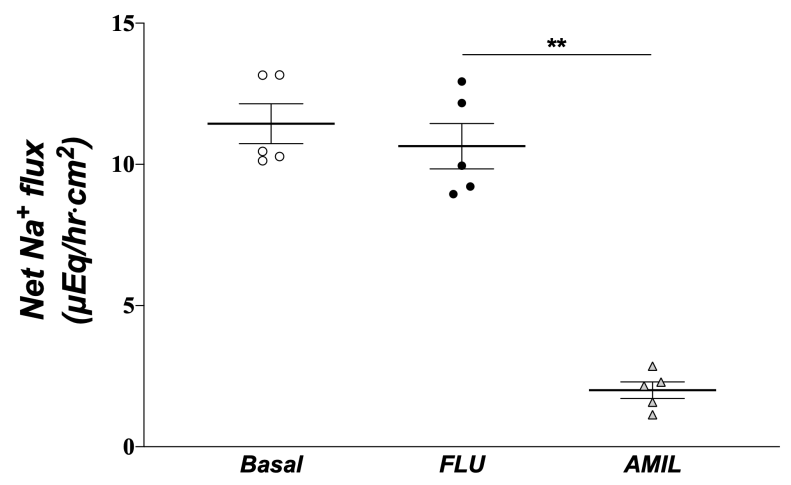

D

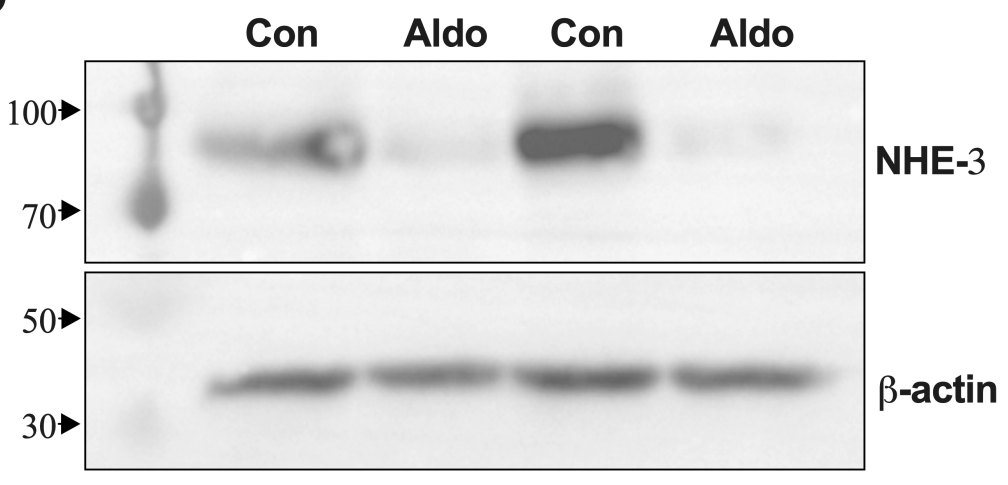

E

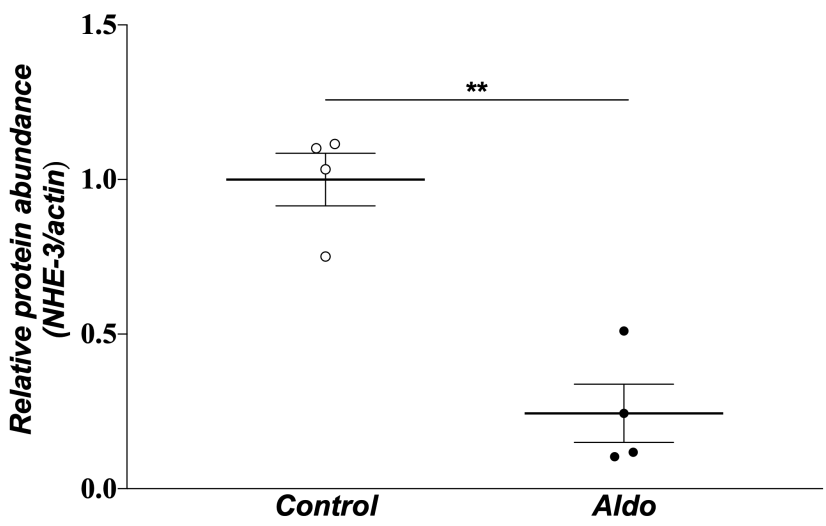


Figure 6 - Flupirtine does not enhance ENaC-mediated $\mathrm{Na}^{+}$absorption in "aldo" rat distal colon. $A-B$ : $\mathrm{I}_{\mathrm{SC}}(A)$ and $\mathrm{G}_{\mathrm{TE}}(B)$ recordings from seromuscular-stripped hyperaldosteronaemic ("aldo") rat distal colon during basal, $100 \mu \mathrm{M}$ serosal flupirtine (FLU) and $10 \mu \mathrm{M}$ apical amiloride (AMIL) flux periods. $C$ : Net ${ }^{22} \mathrm{Na}^{+}$fluxes, measured during the sequential 30 -minute periods shown in $A-B$. D: Representative Western blot showing NHE-3 protein expression in control and "aldo" rat distal colonic epithelium. Protein ladder is shown in the extreme left lane and corresponding molecular weights are given to the left of the blot images. E: Densitometric quantification of two blots similar to the one shown in panel $D(n=4$ separate animals, for both control and "aldo" groups). NHE-3 band intensity was normalized to actin as a loading control and represented as relative abundance in reference to control samples. Lines and error bars represent mean \pm SEM. ${ }^{* *} p<0.01$, as determined by one-way ANOVA with Tukey's post-hoc $(C)$ or unpaired Student's t-test $(E)$. 

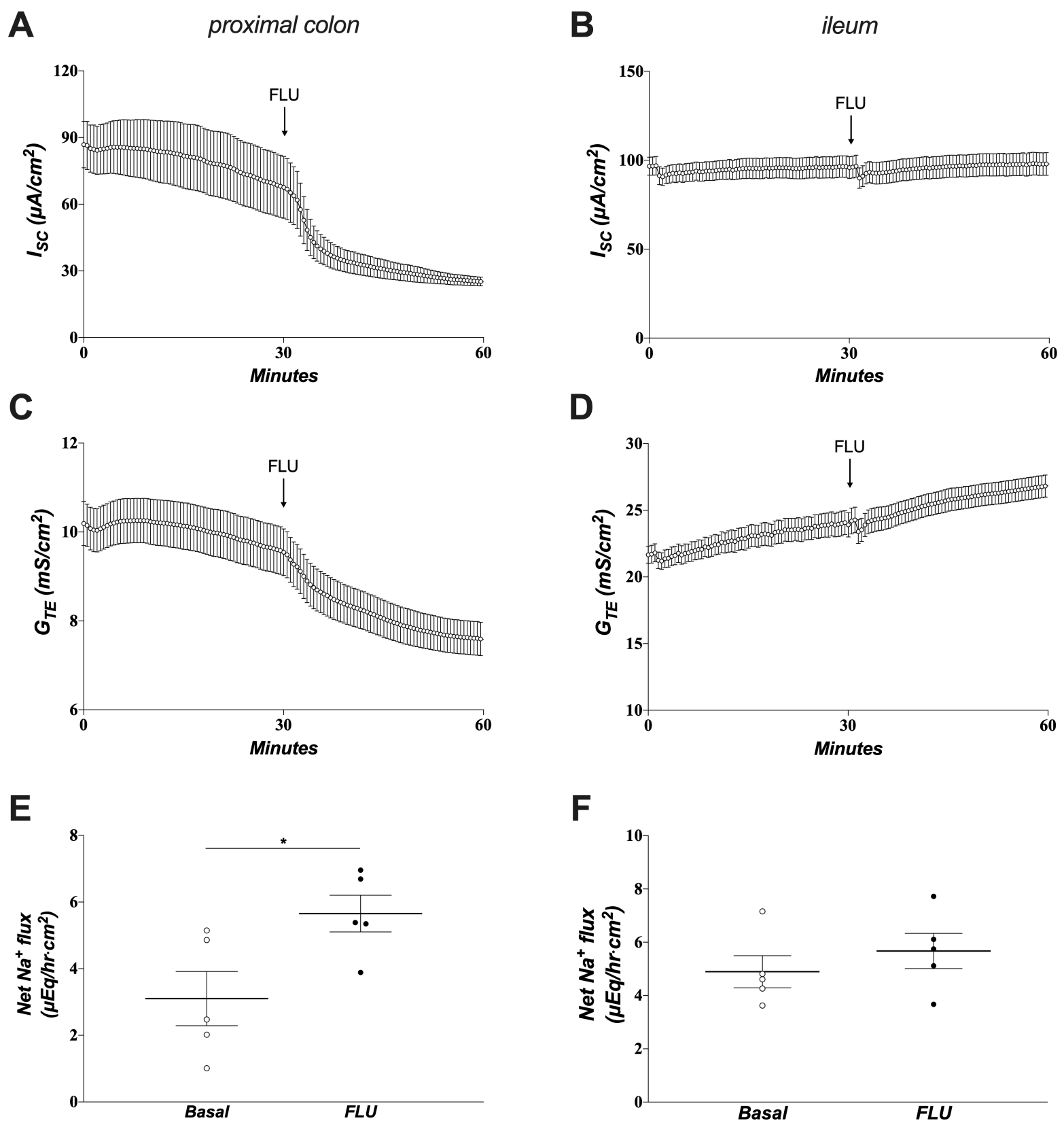

Figure 7 - Flupirtine inhibits $\mathrm{Cl}^{-}$secretion and stimulates $\mathrm{Na}^{+}$absorption in normal rat proximal colon, but not ileum. $A-B$ : $\mathrm{I}_{\mathrm{SC}}$ recordings from seromuscular-stripped rat proximal colon $(A)$ and ileum $(B)$ before and after addition of $100 \mu \mathrm{M}$ flupirtine (FLU) to the serosal chamber bath. $C-D$ : $\mathrm{G}_{\mathrm{TE}}$ recordings from seromuscular-stripped rat proximal colon $(C)$ and ileum (D) before and after addition of $100 \mu \mathrm{M}$ flupirtine (FLU) to the serosal chamber bath. E-F: Net 
${ }^{22} \mathrm{Na}^{+}$fluxes measured in seromuscular-stripped rat proximal colon $(E)$ and ileum $(F)$, corresponding to the sequential 30-minute flux periods before (Basal) and after addition of flupirtine $(F L U)$ as illustrated in panels $A-D$. Lines and error bars represent mean \pm SEM from 5 tissue pairs obtained from 5 separate animals for proximal colon, and 3 separate animals for ileum. ${ }^{*} p<0.05$, as determined by unpaired Student's t-test.

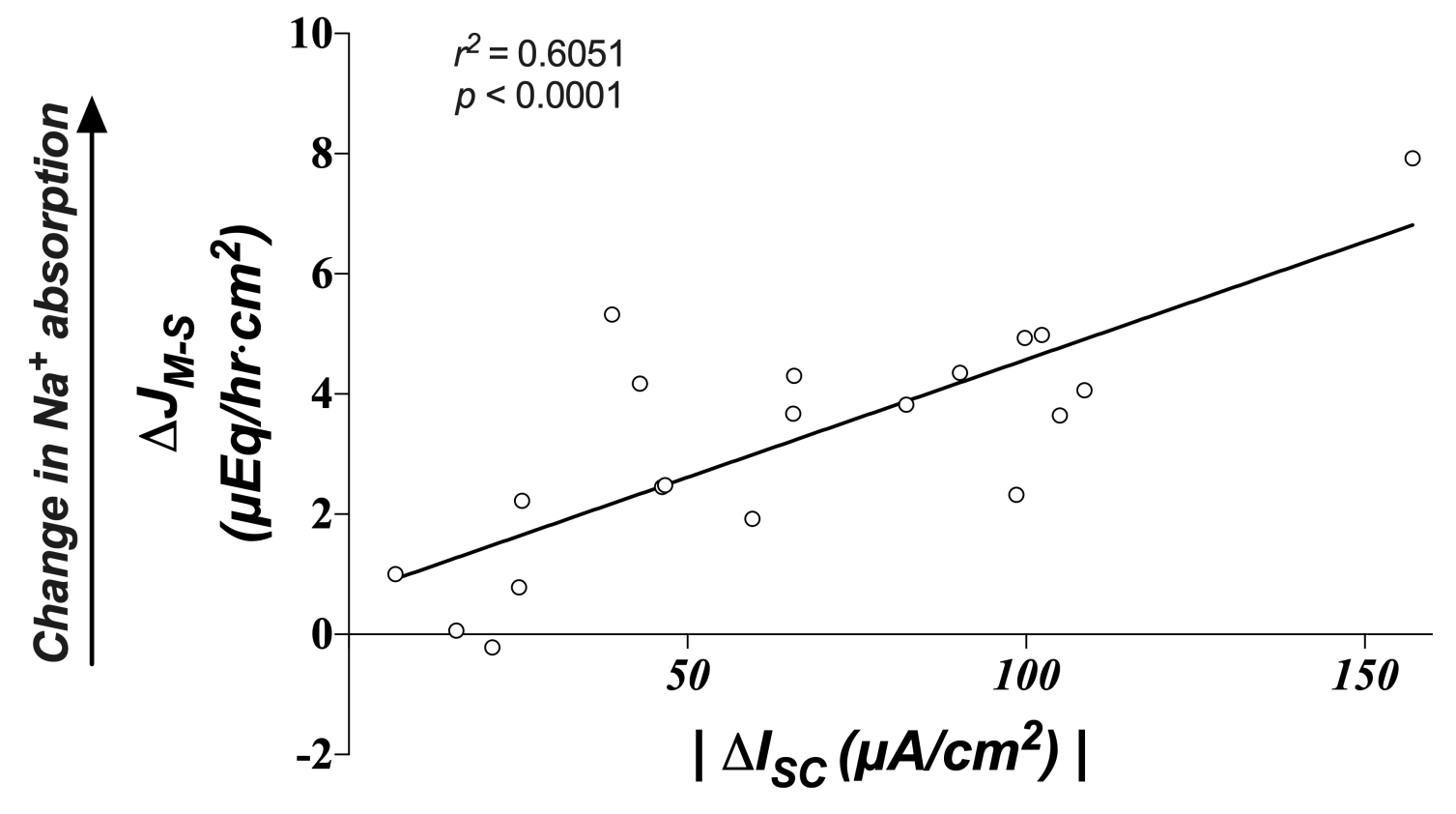

\section{Change in $\mathrm{Cr}$ secretion}

Figure 8 - Stimulation of $\mathrm{Na}^{+}$absorption is significantly correlated to the inhibition of $\mathrm{Cl}^{-}$ secretion in rat colonic tissues treated with flupirtine. The absolute value of the change in $\mathrm{I}_{\mathrm{SC}}$ (inhibition of $\mathrm{Cl}^{-}$secretion) was plotted against change in $\mathrm{M}-\mathrm{S}{ }^{22} \mathrm{Na}^{+}$flux (stimulation of $\mathrm{Na}^{+}$ absorption) from rat colonic tissues that were treated with flupirtine. Every tissue used in this study was included that was assigned as "M-S", where unidirectional ${ }^{22} \mathrm{Na}^{+}$fluxes were measured before and after addition of $100 \mu \mathrm{M}$ flupirtine to the serosal chamber bath $(n=20)$. Simple linear 
regression analysis was performed using GraphPad Prism 9.0 software, and the fit is shown as a solid black line. $p<0.0001$ indicates the line of best fit has a slope significantly greater than zero. 


\section{CHAPTER 4}

TITLE: Aldosterone up-regulates basolateral $\mathrm{Na}^{+}-\mathrm{K}^{+}-2 \mathrm{Cl}^{-}$cotransporter-1 to support enhanced large conductance $\mathrm{K}^{+}$channel-mediated $\mathrm{K}^{+}$secretion in rat distal colon.

SHORT TITLE: Regulation of NKCC1 by aldosterone in colon

Andrew J. Nickerson ${ }^{1}$, Vazhaikkurichi M. Rajendran ${ }^{2,3}$

${ }^{1}$ Department of Physiology, Pharmacology and Neuroscience, West Virginia University School of Medicine, Morgantown, West Virginia

${ }^{2}$ Departments of Biochemistry and ${ }^{3}$ Medicine, West Virginia University School of Medicine, Morgantown, West Virginia

CORRESPONDANCE: Vazhaikkurichi M. Rajendran, PhD, Department of Biochemistry, West Virginia University School of Medicine, 1 Medical Center Drive, Morgantown, WV 26506. Phone: (304) 293-0510; Email: vrajendran@hsc.wvu.edu

ABBREVIATIONS: $\mathrm{I}_{\mathrm{SC}}-$ short circuit current, $\mathrm{G}_{\mathrm{TE}}$ - transepithelial conductance, IbTX iberiotoxin; $\mathrm{BK}$ - large conductance $\mathrm{K}^{+}$channel, IK - intermediate conductance $\mathrm{K}^{+}$channel, Bumet - bumetanide; ESRD - end stage renal disease, $\mathrm{ENaC}$ - epithelial $\mathrm{Na}^{+}$channel.

GRANT SUPPORT: This study was supported by the National Institute of Health NIDDK R01DK104791 and DK112085 grants to VMR.

CONFLICTS OF INTEREST The authors declare no conflicts of interest. 


\section{ABSTRACT}

BACKGROUND AND AIMS: $\mathrm{Na}^{+}-\mathrm{K}^{+}-2 \mathrm{Cl}^{-}$cotransporter-1 (NKCC1) facilitates basolateral $\mathrm{K}^{+}$ and $\mathrm{Cl}^{-}$uptake, supporting their efflux across the mucosal membranes of colonic epithelial cells. NKCC1 activity has also been shown to be critical for electrogenic $\mathrm{K}^{+}$secretion induced by aldosterone, a hormone which is known to stimulate the expression of large conductance $\mathrm{K}^{+}(\mathrm{BK})$ channels in mucosal membranes. This study was aimed to 1) identify whether aldosterone enhances NKCC1 expression specifically to support BK-mediated $\mathrm{K}^{+}$secretion and 2) to determine whether increased NKCC1 supports electrogenic $\mathrm{Cl}$ - secretion in parallel to $\mathrm{K}^{+}$ secretion. METHODS: Dietary $\mathrm{Na}^{+}$depletion was used to induce secondary hyperaldosteronism in rats, or aldosterone was administered ex vivo to rat distal colonic mucosae. NKCC1-dependent electrogenic $\mathrm{K}^{+}$or $\mathrm{Cl}^{-}$secretion was measured as a function of short circuit current $\left(\mathrm{I}_{\mathrm{SC}}\right)$. qRTPCR, western blot and immunofluorescence analyses were performed using standard techniques. RESULTS: Aldosterone enhanced $\mathrm{NKCC} 1$ and $\mathrm{BK} \alpha$ expression and electrogenic $\mathrm{K}^{+}$secretion in the distal colon, which was inhibited by either serosal bumetanide (NKCC1 inhibitor) or mucosal iberiotoxin (IbTX; BK channel blocker), but not TRAM-34 (IK channel blocker). Expression of $\mathrm{NKCC} 1$ and $\mathrm{BK} \alpha$ proteins was enhanced in crypt cells of hyper-aldosterone rats. However, neither NKCC1-dependent $\mathrm{Cl}^{-}$secretion nor CFTR (apical $\mathrm{Cl}^{-}$channel) expression was enhanced by aldosterone. CONCLUSIONS: Aldosterone enhances NKCC1 to support BK-mediated $\mathrm{K}^{+}$ secretion independently of $\mathrm{Cl}^{-}$secretion in the distal colon. The regulation of $\mathrm{NKCC} 1$ expression $/ \mathrm{K}^{+}$secretion by aldosterone may be a therapeutic target in treating gastrointestinal disorders associated with alterations in colonic $\mathrm{K}^{+}$transport, such as colonic pseudo-obstruction, and hyperkalemia associated with end stage renal disease.

Key words: short circuit current, pseudo-obstruction, hyperkalemia, end stage renal disease. 


\section{INTRODUCTION}

The mammalian colon plays a critical role in maintaining body $\mathrm{K}^{+}$homeostasis, as it exhibits both active $\mathrm{K}^{+}$absorptive and secretory processesError! Bookmark not defined.. ${ }^{1,2}$ In the distal colon, $\mathrm{H}^{+}, \mathrm{K}^{+}$-ATPases mediate electroneutral active $\mathrm{K}^{+}$absorption across mucosal membranes $3,4,5,6$, while both $\mathrm{K}^{+}-\mathrm{Cl}^{-}$cotransport (via $\mathrm{KCC} 1$ ) and the parallel function of intermediate conductance $\mathrm{K}^{+}$(IK) channels and $\mathrm{Cl}^{-}$(CLC2) channels have been shown to mediate $\mathrm{K}^{+}$exit across the basolateral membranes in an "electroneutral" manner. ${ }^{7,8}$ Conversely, active $\mathrm{K}^{+}$ secretion in the distal colon is "electrogenic" and mediated primarily by large conductance $\mathrm{K}^{+}$ (BK) channels in the mucosal membranes. ${ }^{1,9,10} \mathrm{The} \mathrm{Na}^{+}, \mathrm{K}^{+}$-ATPase and $\mathrm{Na}^{+}-\mathrm{K}^{+}-2 \mathrm{Cl}^{-}$cotransporter (NKCC1) both contribute to $\mathrm{K}^{+}$uptake across the basolateral membrane, and both transporters are required for supporting electrogenic $\mathrm{K}^{+}$secretion in colon. ${ }^{5,11}$ However, $\mathrm{NKCC1}$ is particularly important to this process, because although its activity is dependent on the $\mathrm{Na}^{+}$gradient established by the $\mathrm{Na}^{+}, \mathrm{K}^{+}$-ATPase, inhibition of $\mathrm{NKCCl}$ alone is sufficient to disrupt $\mathrm{K}^{+}$secretion, while leaving $\mathrm{Na}^{+}, \mathrm{K}^{+}$-ATPase activity intact. ${ }^{11}$

Alterations in colonic $\mathrm{K}^{+}$transport can have a profound impact on disease pathogenicity, either harmful or beneficial. For example, excessive colonic $\mathrm{K}^{+}$secretion is associated with various diarrheal diseases, either secondarily as a result of large stool volume losses, as in $V$. cholera infection $^{12}$, or as a primary driver of watery stool production, as has been suggested in colonic pseudo-obstruction (Ogilvie's syndrome) ${ }^{13,14}$ or ulcerative colitis. ${ }^{15,16}$ On the other hand, increased colonic $\mathrm{K}^{+}$secretion is often a critical adaptation in end stage renal disease (ESRD), providing a secondary outlet for $\mathrm{K}^{+}$from the body, and preventing or delaying life-threatening hyperkalemia. ${ }^{17,18}$ As such, furthering our current understanding of colonic $\mathrm{K}^{+}$transport and its regulation is of high clinical value. 
Aldosterone, a mineralocorticoid hormone, is a major physiological regulator of electrolyte transporters (including $\mathrm{K}^{+}$transporters) in the kidney and colon. ${ }^{11,19,20}$ In addition to its more prominent role in the regulation of $\mathrm{Na}^{+}$absorption, ${ }^{21,22}$ aldosterone is also known to induce active $\mathrm{K}^{+}$secretion by enhancing BK channel expression in the distal colon. ${ }^{15,23,24}$ Aldosterone-induced $\mathrm{K}^{+}$secretion was shown to be dependent on NKCC1 function ${ }^{11}$ and there is evidence to suggest that aldosterone regulates NKCC1 expression, although a direct functional connection between aldosterone-stimulated NKCC1 and BK channels has not yet been established. ${ }^{10,25,26}$ Beyond this, it is unclear whether aldosterone-stimulated NKCC1 activity also supports an enhanced capacity for $\mathrm{Cl}^{-}$secretion. $\mathrm{Cl}^{-}$absorption, which is electroneutral and coupled to $\mathrm{Na}^{+}$absorption in the distal colon, is reduced by aldosterone ${ }^{27}$ However, aldosterone's influence on active (i.e. stimulated) $\mathrm{Cl}^{-}$ secretion - a process dependent on NKCC1 activity - is unknown.

This study was therefore designed to test the hypothesis that aldosterone directly upregulates $\mathrm{NKCC} 1$ in the distal colon, specifically to facilitate active $\mathrm{BK}$-mediated $\mathrm{K}^{+}$secretion, without concurrently enhancing $\mathrm{Cl}^{-}$secretion in the distal colon. Using the model of secondary hyperaldosteronism induced by dietary $\mathrm{Na}^{+}$depletion in rats, or using exogenous aldosterone applied directly to ex vivo tissues, we provide novel evidence that aldosterone increases the expression of $\mathrm{NKCC} 1$ along the crypt/surface axis in the distal colon, and that coordinated NKCC1 and BK channel activity is a key feature of aldosterone-induced colonic $\mathrm{K}^{+}$secretion. Additional studies confirmed that enhanced $\mathrm{NKCC} 1$ expression and activity is specific to the $\mathrm{K}^{+}$transport pathway, as basal and cAMP-stimulated anion $\left(\mathrm{Cl}^{-}\right)$secretion were not altered by aldosterone. Regulation of NKCC1 by aldosterone may therefore be relevant to developing treatment strategies targeting pathologies associated with colonic $\mathrm{K}^{+}$handling, or others involving aberrant NKCC1 function. 


\section{MATERIALS AND METHODS}

Animals: Male and female Sprague-Dawley rats $(200-250 \mathrm{~g})$ were either purchased from Charles River laboratories or bred inhouse (generously provided by Dr. Timothy Nurkiewicz, Department of Physiology and Pharmacology, West Virginia University, Morgantown, WV). All procedures used in this study were approved by WVU's animal care and use committee. Rats were randomly assigned to one of two groups receiving either standard chow or $\mathrm{Na}^{+}$-deficient diet (MP biomedical \#0296023210) for six day to induce secondary hyperaldosteronism. ${ }^{28}$ All rats were given diet and water ad libitum. While anesthetized under 5\% isoflurane, whole colon was removed and washed with ice cold Ringer's solution containing (in $\mathrm{mM}$ ): $140 \mathrm{Na}^{+}, 119.8 \mathrm{Cl}^{-}, 25$ $\mathrm{HCO}_{3}{ }^{-}, 5.2 \mathrm{~K}^{+}, 1.2 \mathrm{Ca}^{2+}, 1.2 \mathrm{Mg}^{2+}, 2.4 \mathrm{HPO}_{4}{ }^{2-}, 0.4 \mathrm{H}_{2} \mathrm{PO}_{4}{ }^{-}$, and 10 glucose (pH 7.4) before being used for mucosal scraping or electrophysiological studies. Euthanasia was then immediately performed by cutting the diaphragm and blood was collected via cardiac puncture.

Aldosterone assay: Serum aldosterone level was determined using an ELISA kit (Invitrogen \#EIAALD). Aldosterone concentration was calculated by fitting sample absorbance values to a standard curve using non-linear regression in GraphPad Prism 8.0 software, as indicated in the kit's instructions.

Mucosal scraping: Distal colon sacs were made by filling with ice cold buffer containing (in $\mathrm{mM}$ ): $15.4 \mathrm{NaCl}, 8 \mathrm{HEPES} /$ Tris (pH 7.5), 5 ethylenediaminetetraacetic acid (EDTA), 0.5 dithiothreitol (DTT), and 0.5 phenylmethylsulphonyl fluoride (PMSF) and incubated in the same solution on ice for 45 minutes. The drained sacs were opened along the mesenteric border. The distal mucosal (epithelial) layer was scraped using glass slides and immediately frozen in liquid nitrogen until use. Scrapped mucosae were used for RNA isolation and protein extraction. 
RNA isolation and $q R T-P C R$ analysis: Total RNA was isolated from frozen colonic mucosa $(50 \mathrm{mg}$ ) using Trizol method and quantitated with NanoDrop spectrometer (Thermo Sci. ND2000C). Quantitative reverse transcriptase polymerase chain reaction (qRT-PCR) was performed using One-Step kit (NEB \#E3005) per the manufacturer's protocol. In brief, custom gene-specific primers were designed using the published DNA sequence and synthesized by Thermo Scientific, and are listed below in Table 1.

Gene Target and

Accession number

Primer Sequence Amplicon length

NKCC1 (Slc12a2) F: 5'- TGGCAAGACTTCAACTCAGC - 3'

NM 001270617.1 R: 5'- GGTATCAACAAGGTCAAACCTCC - 3'

177

$E N a C \gamma($ Scnnlg) F: 5'-ATGATACCTCTGACTGCGCC - 3'

NM_017046.2 R: 5'-AAAAGCGTGAAGTTCCTGGC - 3'

199

BK $\alpha$ (Kcnmal) F: 5'- ATGTCTACAGTGGGTTACGG - 3'

NM_001393699.1 R: 5'- TGGGTGGTAGTTCTTTATGG - 3'

464

NaKa (ATPlal) F: 5'- CATCAACGCAGAGGATGTCG - 3'

NM_012504.1 R: 5'- AGTGAGGAGTTATCCACCTTGC - 3'

CFTR (Cftr) F: 5'- TTCTTCAGCTGGACCACACC - 3'

NM_031506.1 R: 5'- TGGAAGCTTGTTCCCTGTCC - 3'

106

$\beta$-actin (Actb) F: 5'-AGATCAAGATCATTGCTCCTCC - 3'

NM_031144.3 R: 5'-AGTAACAGTCCGCCTAGAAGC - 3'

Table 1: Primer sequences used in qRT-PCR analysis of RNA extracts from rat distal colon.

qRT-PCR reactions performed on a 96-well plate were read using a CFX96 real time PCR machine (BioRad). Differential mRNA abundance was determined using the delta-delta $C_{t}$ method, with $\beta$-actin as a reference housekeeping gene. 
Western blot analysis: Proteins were extracted from frozen colonic mucosa $(50 \mathrm{mg})$ by homogenizing in lysis buffer $(150 \mathrm{mM} \mathrm{NaCl}, 50 \mathrm{mM}$ Tris base, $0.5 \%$ sodium deoxycholate, $0.1 \%$ SDS, $1.0 \%$ Triton-X100) plus protease inhibitor cocktail (Thermo A32965), followed by a brief sonication (Fisher Sonic Dismembranator Model 100). Following centrifugation, the supernatant was mixed with 4x Laemmli buffer (Life Technologies NP0007) and heated to $90^{\circ} \mathrm{C}$ for 5 minutes. Samples were then chilled on ice before adding $\beta$-mercaptoethanol $(5 \% \mathrm{w} / \mathrm{v})$, and the aliquoted samples were flash frozen and stored at $-80^{\circ} \mathrm{C}$. Proteins $(\sim 20 \mu \mathrm{g})$ were resolved on $8 \%$ Tris-glycine polyacrylamide gels and transferred on to PVDF membranes. Membranes were incubated in blocking buffer (3\% BSA in tris-buffered saline plus $0.1 \%$ Tween-20 (TBS-T) for 1 hour at room temperature, then incubated overnight with either rabbit anti-NKCC1 (Cell Signaling Technologies 14581, 1:1000) ${ }^{29}$ or horseradish peroxidase-conjugated rabbit anti-actin (Santa Cruz Biotechnologies SC-4778, 1:1000) at $4^{\circ} \mathrm{C}$. The membranes were then washed in TBST (5 minutes x5). Blots incubated with anti-NKCC1 were incubated with secondary antibody solution containing goat anti-rabbit (Thermo 31462, 1:20,000) for 1 hour at room temperature and washed again in TBST ( 5 min x 5). Immune complexes were detected using enhanced chemiluminescence (West Dura, Thermo 34075). Images were captured using a G:BOX (Syngene) and band intensities were quantified by densitometry using Image $J$ software and normalized to $\beta$-actin as a loading control.

Immunofluorescence studies: Distal colon segments were fixed in 4\% paraformaldehyde in PBS overnight at $4^{\circ} \mathrm{C}$, washed in PBS, and flash frozen in isopentane cooled with liquid nitrogen before embedding in tissue freezing medium (Thermo 1437365) and storing at $-80^{\circ} \mathrm{C}$. Sections $(8$ $\mu \mathrm{m})$ were cut and mounted on charged glass slides. Antigen retrieval was then performed by heating to $70^{\circ} \mathrm{C}$ in $10 \mathrm{mM}$ citrate ( $\mathrm{pH}$ 6.0) for 40 minutes using a microwave on low power. After 
cooling, sections were permeabilized and blocked in 5\% goat serum and $0.1 \%$ Tween-20 in PBS (PBST) for 30 minutes, then incubated in blocking solution containing rabbit anti-NKCC1 (1:500) and mouse anti-BK $\alpha$ (Millipore MABN70, 1:100) $)^{\text {so }}$ antibodies overnight at $4^{\circ} \mathrm{C}$. The following day, sections were washed in PBST (5 minutes x 5) and incubated for 1 hour at room temperature in blocking solution containing Alexa Fluor 488 goat anti-rabbit (Thermo A11008) and Alexa Fluor 568 goat anti-mouse (Thermo A11031). After washing again in PBST, coverslips were mounted with SlowFade Diamond mountant containing DAPI (Thermo S36973) and sealed with clear nail polish. Images were captured with a Zeiss 710 confocal microscope (Carl Zeiss, NY).

Ussing chamber studies: Distal colon segments placed in ice cold Ringer's solution containing indomethacin $(5 \mu \mathrm{M} \text {; to inhibit prostaglandin production })^{31}$ were opened along the mesenteric border and the mucosal layers were separated, as described previously ${ }^{8}$. Briefly, the colons were placed in a dissecting dish and a shallow lateral incision was made into the mucosal layer just proximal to the most distal Peyer's patch ( $2 \mathrm{~cm}$ from the rectum). The distal mucosal layer (2-6 cm proximal to the rectum) was then separated from the underlying smooth muscle layers. Mucosae mounted on EasyMount sliders $\left(1.1 \mathrm{~cm}^{2}\right.$ aperture) placed Ussing Style chambers (Physiologic Instruments, San Diego CA) were bathed bilaterally with Ringer's solution, bubbled with $5 \% \mathrm{CO}_{2}$ balanced with oxygen, and warmed to $37^{\circ} \mathrm{C}$. Tetrodotoxin $(1 \mu \mathrm{M})$ was added to the serosal bath to inhibit residual neuronal activity ${ }^{31}$. Tissues were allowed to equilibrate until transepithelial potential $\left(\mathrm{V}_{\mathrm{TE}}\right)$ had stabilized ( $\sim 20$ minutes). Then, tissues were voltage clamped and short-circuit current $\left(\mathrm{I}_{\mathrm{SC}}\right)$ was measured by computer-operated software (Physiologic Instruments, San Diego CA). For all data presented here, positive $\mathrm{I}_{\mathrm{SC}}$ reflect anion secretion (serosal to mucosal direction) and/or cation absorption (mucosal to serosal direction). Trans-epithelial conductance $\left(\mathrm{G}_{\mathrm{TE}}\right)$ was monitored by generating a bipolar $5 \mathrm{mV}$ pulse at 5 second intervals. All tissues were 
allowed to reach steady-state $\mathrm{I}_{\mathrm{SC}}$ (i.e., plateau) for at least 10 minutes prior to the addition of any drugs.

\section{RESULTS}

Dietary $\mathrm{Na}^{+}$depletion induces secondary hyperaldosteronism in a sex-dependent manner.

Dietary $\mathrm{Na}^{+}$depletion is commonly used to generate experimental hyperaldosteronism. ${ }^{27,32,33}$ In the past, the effects of experimental hyper-aldosteronism on kidney and colon electrolyte transport have been mostly characterized in male, but not in female animals. However, in accordance with current NIH guidelines regarding biological variability related to sex, we examined the effect of dietary $\mathrm{Na}^{+}$depletion on plasma aldosterone levels in both male and female rats. Although the serum aldosterone levels were similar in both normal male and female $(244.9 \pm 29.9$ vs $245.9 \pm 61.7 \mathrm{pg} / \mathrm{mL})$, dietary $\mathrm{Na}^{+}$depletion induced a 14.2 -fold increase in serum aldosterone level in male rats (normal vs $\mathrm{Na}^{+}$-depleted: $244.9 \pm 29.9$ vs. $3,485.0 \pm 949.1$ $\mathrm{pg} / \mathrm{mL}$ ), but only a 3.4-fold increase in female rats (normal vs $\mathrm{Na}^{+}$-depleted: $245.9 \pm 61.7$ vs. 827.5 $\pm 57.3 \mathrm{pg} / \mathrm{mL}$ ) (Supp. Fig. 1A).

Because dietary $\mathrm{Na}^{+}$depletion-induced hyperaldosteronism is known to induce epithelial $\mathrm{Na}^{+}$channel (ENaC)-mediated $\mathrm{Na}^{+}$absorption ${ }^{21,22}$, we also examined whether the differentiallyincreased aldosterone levels were reflected in $\mathrm{ENaC}$ activity in male and female rat distal colon. Similar to the difference observed regarding aldosterone generation, $\mathrm{Na}^{+}$depletion induced $\mathrm{ENaC}$ activity proportionately lower ( $\sim 4$-fold) in dietary $\mathrm{Na}^{+}$-depleted female compared to male rat distal colon, as measured by $100 \mu \mathrm{M}$ amiloride-sensitive short circuit current $\left(\mathrm{I}_{\mathrm{SC}} ; \mathrm{ENaC}\right.$-mediated $\mathrm{Na}^{+}$ absorption) $\left(\mathrm{Na}^{+}\right.$-depleted male vs female: $\Delta \mathrm{I}_{\mathrm{SC}(\mathrm{AMIL})}=-388.3 \pm 28.3$ vs $\left.-101.2 \pm 31.6 \mu \mathrm{A} / \mathrm{cm}^{2}\right)$ (Supp. Fig. 1B). A concentration of $100 \mu \mathrm{M}$ amiloride was selected based on similar published 
experiments. ${ }^{23,24}$ To assess whether a sex-specific effect of dietary $\mathrm{Na}^{+}$depletion had occurred, a Two-way analysis of variance (ANOVA) with Tukey's post-hoc was performed. Analysis showed significantly elevated serum aldosterone level in dietary $\mathrm{Na}^{+}$-depleted male $(p<0.01)$, but not in female $(p=0.82)$ rats; and significantly induced ENaC activity in dietary $\mathrm{Na}^{+}$-depleted male $(p<$ $0.001)$, but not female $(p=0.06)$ rat distal colon.

Although serum aldosterone and $\mathrm{ENaC}$ activity were appreciably high in dietary $\mathrm{Na}^{+}-$ depleted female rats, and thus the dietary effects are certainly not negligible, the response was not as robust as in the males and therefore requires separate independent study to identify the mechanistic nature of lower aldosterone generation level in $\mathrm{Na}^{+}$-depleted female rats. However, as the specific goal of this investigation is to evaluate the regulation of $\mathrm{NKCC} 1$ and $\mathrm{BK}$-mediated $\mathrm{K}^{+}$ secretion by aldosterone, all subsequent experiments were designed to incorporate only male rats to avoid confounding effects resulting from sex differences, as well as to maintain consistency with existing literature regarding aldosterone-regulated $\mathrm{K}^{+}$transport.

Dietary $\mathrm{Na}^{+}$depletion stimulates electrogenic $\mathrm{Na}^{+}$absorption and $\mathrm{K}^{+}$secretion in rat distal colon.

Further initial studies were performed to confirm that dietary $\mathrm{Na}^{+}$depletion induces $\mathrm{ENaC}$ mediated electrogenic $\mathrm{Na}^{+}$absorption and BK channel-mediated electrogenic $\mathrm{K}^{+}$secretion in rat distal colon, as has been previously reported. ${ }^{24,34}$ As shown in Supplemental Fig. 2A, the large, positive basal $\mathrm{ISC}_{\mathrm{SC}}$ measured in $\mathrm{Na}^{+}$-depleted rat distal colon indicates the presence of $\mathrm{ENaC}$ activity (normal vs dietary $\mathrm{Na}^{+}$-depleted: basal $\mathrm{I}_{\mathrm{SC}}=28.3 \pm 3.2$ vs $353.8 \pm 8.9 \mu \mathrm{A} / \mathrm{cm}^{2} ; p<0.001$ ), while inhibition by mucosal amiloride further confirmed that $\mathrm{ENaC}$ activity was the source of this basal $\mathrm{I}_{\mathrm{SC}}$ in dietary- $\mathrm{Na}^{+}$depleted rat distal colon (normal vs dietary $\mathrm{Na}^{+}$-depleted: $\mathrm{I}_{\mathrm{SC}}$ post-amiloride $=$ $17.9 \pm 0.9$ vs $\left.-40.0 \pm 3.3 \mu \mathrm{A} / \mathrm{cm}^{2} ; p<0.001\right)$. Negative $\mathrm{I}_{\mathrm{SC}}$ in the presence of amiloride indicates electrogenic $\mathrm{K}^{+}$secretion (Fig. 2A). Lower basal $\mathrm{I}_{\mathrm{SC}}$ that was less sensitive to amiloride, as well 
as a positive residual $\mathrm{I}_{\mathrm{SC}}$ after amiloride application, suggested that normal distal colon does not exhibit a significant amount of electrogenic $\mathrm{Na}^{+}$absorption and $\mathrm{K}^{+}$secretion, respectively. The positive $\mathrm{I}_{\mathrm{SC}}$ in normal rat distal colon likely represents anion $\left(\mathrm{Cl}^{-} / \mathrm{HCO}_{3}{ }^{-}\right)$secretion (Supp. Fig. 2A). A parallel increase in $\mathrm{ENaC} \gamma$ subunit (12.5-fold), as well as $\mathrm{BK} \alpha$ subunit (2.3-fold) specific mRNA abundance indicated that $\mathrm{ENaC}$ and $\mathrm{BK}$ channel expression were induced in dietary $\mathrm{Na}^{+}-$ depleted rat distal colon (Supp. Fig. 2B), consistent with the observed functional increase in both electrogenic $\mathrm{Na}^{+}$absorption and electrogenic $\mathrm{K}^{+}$secretion in distal colon from normal versus $\mathrm{Na}^{+}-$ depleted rats. These data validate the model of secondary hyperaldosteronism by $\mathrm{Na}^{+}$deprivation used in the experiments to follow.

Dietary $\mathrm{Na}^{+}$depletion stimulates $\mathrm{NKCCl}$ expression in rat distal colon.

qRT-PCR analyses were performed to determine whether, in parallel to BK $\alpha$ mRNA, the abundance of basolateral $\mathrm{K}^{+}$transporters, $\mathrm{Na}^{+}, \mathrm{K}^{+}$-ATPase and $\mathrm{NKCC} 1$ were also increased in dietary $\mathrm{Na}^{+}$-depleted rat distal colon. NKCC1 (2.2-fold; $p<0.01$ ), but not $\mathrm{NaK} \alpha$ (catalytic subunit of $\mathrm{Na}^{+}, \mathrm{K}^{+}$-ATPase) specific mRNA abundance was significantly increased (Fig. 1A). Previous studies have also found that $\mathrm{Na}^{+}, \mathrm{K}^{+}$-ATPase subunits are not transcriptionally regulated by aldosterone, but rather an increase in activity is achieved via an increase in transporter density within the basolateral membrane ${ }^{35,36}$. A single product of predicted size (177 bp) confirmed the specific amplification of NKCC1 mRNA in the qRT-PCR reactions (Fig. 1B). Western blot analysis also showed increased $\mathrm{NKCC} 1$ protein expression in dietary $\mathrm{Na}^{+}$-depleted compared to normal rat distal colon (2.3-fold; $p<0.01)$ (Figs. 1C and 1D).

Dietary $\mathrm{Na}^{+}$depletion induces bumetanide-sensitive $\mathrm{K}^{+}$secretion in rat distal colon. 
Experiments were next performed to establish that basolateral $\mathrm{K}^{+}$uptake via $\mathrm{NKCC1}$ supports electrogenic $\mathrm{K}^{+}$secretion in $\mathrm{Na}^{+}$-depleted rat distal colon. The contribution of NKCC1supported $\mathrm{K}^{+}$secretion was measured as a function of bumetanide-sensitive $\mathrm{I}_{\mathrm{SC}}$ in an Ussing-style recording chamber. Bumetanide (NKCC1-specific inhibitor; $200 \mu \mathrm{M}$ ) -sensitive $\mathrm{I}_{\mathrm{SC}}$ was measured in the presence of mucosal amiloride $(100 \mu \mathrm{M})$, to eliminate interference by highly electrogenic ENaC activity. A concentration of $200 \mu \mathrm{M}$ bumetanide was selected based on previous literature. ${ }^{11,24}$ In the presence of amiloride, positive and negative $\mathrm{I}_{\mathrm{SC}}$ values were measured in normal and dietary $\mathrm{Na}^{+}$-depleted rat distal colon, respectively (normal vs dietary $\mathrm{Na}^{+}$-depleted: $\mathrm{I}_{\mathrm{SC}}$ $\left.=16.8 \pm 0.7 \mathrm{vs}-39.9 \pm 9.8 \mu \mathrm{A} / \mathrm{cm}^{2} ; p<0.01\right)$ (Figs. $2 \mathrm{~A}$ and $2 \mathrm{C}$ ). Application of bumetanide to the serosal bath induced a reversal from negative to positive $\mathrm{I}_{\mathrm{SC}}$ in $\mathrm{Na}^{+}$-depleted rat distal colon (basal vs bumetanide: $\mathrm{I}_{\mathrm{SC}}=-39.9 \pm 4.4$ vs $7.1 \pm 1.7 \mu \mathrm{A} / \mathrm{cm}^{2} ; p<0.01$ ), while having no significant effect in normal distal colon (basal vs bumetanide: $\mathrm{I}_{\mathrm{SC}}=16.8 \pm 0.7 \mathrm{vs} 13.0 \pm 0.7 \mu \mathrm{A} / \mathrm{cm}^{2} ; p=0.74$ ) (Fig. $2 \mathrm{~A}$ and $2 \mathrm{C}$ ). The average change in $\mathrm{I}_{\mathrm{SC}}$ induced by bumetanide was significantly larger in $\mathrm{Na}^{+}-$ depleted vs normal rat distal colon (normal vs $\mathrm{Na}^{+}$-depleted: $\Delta \mathrm{I}_{\mathrm{SC}(\mathrm{BUMET})}=-4.6 \pm 0.8$ vs $47.0 \pm 3.1$ $\left.\mu \mathrm{A} / \mathrm{cm}^{2} ; p<0.01\right)$ (Fig. 2E).

Serosal application of bumetanide also significantly inhibited $\mathrm{G}_{\mathrm{TE}}$ in dietary $\mathrm{Na}^{+}$-depleted (basal vs bumetanide: $\mathrm{G}_{\mathrm{TE}}=6.2 \pm 0.5$ vs. $4.2 \pm 0.4 \mathrm{mS} / \mathrm{cm}^{2} ; p<0.01$ ), but not normal (basal vs bumetanide: $4.6 \pm 0.2$ vs. $4.3 \pm 0.2 \mathrm{mS} / \mathrm{cm}^{2} ; p=0.26$ ) rat distal colon (Fig. $2 \mathrm{~B}$ and $2 \mathrm{D}$ ), suggesting the positive change in $\mathrm{I}_{\mathrm{SC}}$ induced by bumetanide was due to a reduction in cation $\left(\mathrm{K}^{+}\right)$secretion rather than an increase in anion $\left(\mathrm{Cl}^{-} / \mathrm{HCO}_{3}{ }^{-}\right)$secretion or cation $\left(\mathrm{Na}^{+}\right)$absorption. The average change in $\mathrm{G}_{\mathrm{TE}}$ induced by bumetanide was also significantly greater in $\mathrm{Na}^{+}$-depleted vs normal rat distal colon (normal vs $\mathrm{Na}^{+}$-depleted: $\Delta \mathrm{G}_{\mathrm{TE}(\mathrm{BUMET})}=-0.01 \pm 0.04 \mathrm{vs}-2.0 \pm 0.2 \mathrm{mS} / \mathrm{cm}^{2} ; p<0.01$ ) (Fig. 2F). These observations suggest that $\mathrm{NKCC1}$ functions as a major basolateral $\mathrm{K}^{+}$loader 
during electrogenic $\mathrm{K}^{+}$secretion in dietary $\mathrm{Na}^{+}$-depleted rat distal colon, while having little effect on basal secretion in normal distal colon.

Ex vivo aldosterone induces bumetanide-sensitive $\mathrm{K}^{+}$secretion and $N K C C 1$ expression in normal rat distal colon.

To establish that aldosterone, but not the dietary $\mathrm{Na}^{+}$depletion per se, is critical for induced electrogenic $\mathrm{K}^{+}$secretion and enhanced $\mathrm{NKCC} 1 / \mathrm{BK} \alpha$ expression, the effect of aldosterone applied ex vivo was examined in normal rat distal colon mucosae mounted in the recording chamber. Similar to dietary $\mathrm{Na}^{+}$depletion, ex vivo aldosterone $(10 \mathrm{nM}$, based upon measured serum aldosterone levels) over an 8-hour incubation period induced both $\mathrm{ENaC}$ activity (i.e., increased amiloride-sensitive $\mathrm{I}_{\mathrm{SC}}$ ), as well as electrogenic $\mathrm{K}^{+}$secretion (i.e., negative $\mathrm{I}_{\mathrm{SC}}$ ) in presence of amiloride, which were not observed in control tissues or tissues treated with aldosterone plus 10 $\mu \mathrm{M}$ spironolactone, a mineralocorticoid antagonist (Fig. 3A-B). It should be noted that colonic mucosae remain viable for many hours under these conditions, as previously demonstrated. ${ }^{37}$ Serosal addition of bumetanide completely inhibited the aldosterone-induced electrogenic $\mathrm{K}^{+}$ secretion (basal vs bumetanide: $\mathrm{I}_{\mathrm{SC}}=-29.9 \pm 1.3$ vs $8.6 \pm 1.9 \mu \mathrm{A} / \mathrm{cm}^{2} ; p<0.01$ ). Bumetanidesensitive ISC was significantly greater in aldosterone-treated versus control tissues (control vs. aldosterone-treated: $\Delta \mathrm{I}_{\mathrm{SC}(\mathrm{BUMET})}=-5.7 \pm 1.4$ vs. $\left.30.9 \pm 1.3 \mu \mathrm{A} / \mathrm{cm}^{2} ; p<0.001\right)$ and aldosterone alone compared to aldosterone plus spironolactone-treated tissues (aldosterone vs. aldosterone + spironolactone: $\Delta \mathrm{I}_{\mathrm{SC}(\mathrm{BUMET})}=-4.0 \pm 1.1$ vs. $\left.30.9 \pm 1.3 \mu \mathrm{A} / \mathrm{cm}^{2} ; p<0.001\right)$ (Fig. 3C). Bumetanidesensitive- $\mathrm{G}_{\mathrm{TE}}$ followed the same pattern (control vs. aldosterone-treated: $\Delta \mathrm{G}_{\mathrm{TE}(\mathrm{BUMET})}=-0.2 \pm 0.02$ vs. $\left.-1.0 \pm 0.04 \mathrm{mS} / \mathrm{cm}^{2} ; p<0.001\right) ;\left(\right.$ aldosterone vs. aldosterone + spironolactone $: \Delta \mathrm{G}_{\mathrm{TE}(\mathrm{BUMET})}=$ $-0.2 \pm 0.01$ vs. $-1.0 \pm 0.04 \mathrm{mS} / \mathrm{cm}^{2} ; p<0.001$ ) (Fig. 3D). qRT-PCR analyses performed on total 
RNA isolated from the same tissues revealed that aldosterone treatment significantly increased the NKCC1 (1.8-fold; $p<0.05)$ and BK $\alpha$ (5.9-fold; $p<0.01)$ specific mRNA abundance compared to control or aldosterone plus spironolactone-treated tissues (Fig. 3E). Interestingly, BK $\alpha$ mRNA abundance was still increased in aldosterone plus spironolactone-treated tissues compared to control (2.7-fold; $p<0.05)$, suggesting either that $10 \mu \mathrm{M}$ spironolactone is insufficient to completely inhibit $\mathrm{BK} \alpha$ induction by aldosterone, or that aldosterone-induced $\mathrm{BK} \alpha$ expression may be partially mediated by a spironolactone-insensitive mechanism. Nonetheless, these data indicate that the increased serum aldosterone, but not the dietary $\mathrm{Na}^{+}$depletion alone, was likely responsible for the enhanced $\mathrm{NKCC} 1$ and $\mathrm{BK} \alpha$ expression and electrogenic $\mathrm{K}^{+}$secretion in rat distal colon observed in earlier experiments.

Bumetanide-sensitive electrogenic $K^{+}$secretion is mediated by mucosal BK channels in dietary $\mathrm{Na}^{+}$-depleted rat distal colon.

We have previously found increased mucosal BK and IK channel activities in dietary $\mathrm{Na}^{+}-$ depleted rat distal colon. ${ }^{37}$ Thus, to identify whether BK and/or IK channels mediate bumetanidesensitive $\mathrm{K}^{+}$exit across mucosal membranes, the effect of mucosal $\mathrm{K}^{+}$channel blockers $\mathrm{Ba}^{2+}(\mathrm{a}$ nonspecific $\mathrm{K}^{+}$channel blocker), Iberiotoxin (IbTX; a selective BK channel blocker) and Tram-34 (a selective IK channel blocker) were examined. $\mathrm{K}^{+}$channel blocker concentrations were selected based on similar experiments described in the literature. ${ }^{9,38}$ Mucosal $\mathrm{Ba}^{2+}(3 \mathrm{mM})$ inhibited basal $\mathrm{K}^{+}$secretion $\left(\Delta \mathrm{I}_{\mathrm{SC}(\mathrm{Ba} 2+)}=19.8 \pm 3.1 \mu \mathrm{A} / \mathrm{cm}^{2}\right)($ Fig. $4 \mathrm{~A}, \mathrm{D})$ and significantly attenuated the effect of bumetanide in $\mathrm{Na}^{+}$-depleted rat distal colon (no inhibitor vs. $\mathrm{Ba}^{2+}: \Delta \mathrm{I}_{\mathrm{SC}(\mathrm{BUMET})}=47.0 \pm 3.1$ vs. $\left.19.3 \pm 2.5 \mu \mathrm{A} / \mathrm{cm}^{2} ; p<0.001\right)(\mathrm{Fig}, 4 \mathrm{~F})$. Mucosal IbTX also inhibited basal $\mathrm{K}^{+}$secretion $\left(\Delta \mathrm{I}_{\mathrm{SC}(\mathrm{IbTx})}\right.$ $\left.=33.8 \pm 4.0 \mu \mathrm{A} / \mathrm{cm}^{2}\right)($ Fig. $4 \mathrm{~B}, \mathrm{D})$ and attenuated the effect of serosal bumetanide as well (no 
inhibitor vs. IbTX: $\Delta \mathrm{I}_{\mathrm{SC}(\mathrm{BUMET})}=47.0 \pm 3.1$ vs. $\left.15.9 \pm 1.8 \mu \mathrm{A} / \mathrm{cm}^{2} ; p<0.001\right)$ (Fig. $4 \mathrm{~F}$ ), although a much longer time period was required to achieve inhibition with IbTX, as is expected. ${ }^{23}$ Addition of mucosalTram-34 $(250 \mathrm{nM})$ produced no appreciable change in basal $\mathrm{K}^{+}$secretion $\left(\Delta \mathrm{I}_{\mathrm{SC}(\mathrm{TRAM}-34)}\right.$ $=-1.7 \pm 1.8 \mu \mathrm{A} / \mathrm{cm}^{2}$ ) (Fig. 4 C,D) and did not alter the response to serosal bumetanide (no inhibitor vs. Tram-34: $\Delta \mathrm{I}_{\mathrm{SC}(\mathrm{BUMET})}=47.0 \pm 3.1$ vs. $44.5 \pm 4.2 \mu \mathrm{A} / \mathrm{cm}^{2} ; p>0.98$ ) (Fig. $\left.4 \mathrm{~F}\right)$. Together, these data suggest that NKCC1-dependent $\mathrm{K}^{+}$secretion in $\mathrm{Na}^{+}$-depleted rat distal colon is likely mediated by mucosal membrane BK, but not IK channels.

Addition of $\mathrm{Ba}^{2+}$ (non-selective $\mathrm{K}^{+}$channel blocker) produced a similar effect to IbTX with respect to inhibition of basal $\mathrm{K}^{+}$secretion (Fig. 4C), although IbTX-induced inhibition was significantly greater than that of $\mathrm{Ba}^{2+}\left(\Delta \mathrm{I}_{\mathrm{SC}(\mathrm{IbTX})}\right.$ vs. $\Delta \mathrm{I}_{\mathrm{SC}(\mathrm{Ba} 2+)}=33.8 \pm 4.0$ vs. $19.8 \pm 3.1 \mu \mathrm{A} / \mathrm{cm}^{2}$; $p<0.05$ ) (Fig. 4D). However, residual $\mathrm{I}_{\mathrm{SC}}$ following IbTX or $\mathrm{Ba}^{2+}$ administration was not different (IbTX vs. $\mathrm{Ba}^{2+}: \mathrm{I}_{\mathrm{SC}(\mathrm{RES})}=-8.8 \pm 2.4$ vs. $-12.9 \pm 2.5 \mu \mathrm{A} / \mathrm{cm}^{2} ; p=0.61$ ) (Fig. 4E), suggesting this difference may have resulted from variability in the initial $I_{S C}$ values. In the continued presence of mucosal $\mathrm{Ba}^{2+}$, IbTX or TRAM-34, serosal bumetanide completely inhibited the electrogenic $\mathrm{K}^{+}$ secretion and unmasked the apparent presence of a small degree of anion secretion (i.e. positive ISC) (Fig. 4A-C), similar to Figures 3 and 4. It is also to be noted that only a negligible amount of IbTX-sensitive $\mathrm{K}^{+}$secretion was present in normal compared to dietary $\mathrm{Na}^{+}$-depleted rat distal colon (normal vs dietary $\mathrm{Na}^{+}$-depleted: $\Delta \mathrm{I}_{\mathrm{SC}(\mathrm{IbTX})}=3.1 \pm 1.2$ vs. $33.8 \pm 4.0 \mu \mathrm{A} / \mathrm{cm}^{2} ; p<0.001$ ), supporting the earlier finding that $\mathrm{BK} \alpha$ subunits expression is upregulated by dietary $\mathrm{Na}^{+}$depletion (Supp. Fig. 2).

Unexpectedly, serosal bumetanide appeared to inhibit residual $\mathrm{K}^{+}$secretion even in the presence of the non-selective $\mathrm{K}^{+}$channel blocker, $\mathrm{Ba}^{2+}$. Incomplete inhibition of apical $\mathrm{K}^{+}$ channels may have resulted from the effective (free) $\mathrm{Ba}^{2+}$ concentration being reduced by mucins 
or carbonate anions present in the apical bath, or from tissue architecture impeding the diffusion of drugs to the target cell membranes. Nonetheless, the inhibitory effect of bumetanide on basal $\mathrm{K}^{+}$secretion was substantially diminished by $\mathrm{IbTX}$ and $\mathrm{Ba}^{2+}(60-70 \%)$, suggesting that coordinated uptake via $\mathrm{NKCC} 1$ at the basolateral membrane and exit via BK channels at the apical membrane are both essential for basal secretion of $\mathrm{K}^{+}$induced by aldosterone in rat distal colon.

Dietary $\mathrm{Na}^{+}$depletion enhances $\mathrm{NKCC1}$ and BKa channel protein expression together in rat distal colonic crypts.

Inhibition of basal electrogenic $\mathrm{K}^{+}$secretion by bumetanide and/or IbTX suggested that basolateral $\mathrm{NKCC} 1$ and mucosal $\mathrm{BK}$ channels may be coordinately regulated by aldosterone in dietary $\mathrm{Na}^{+}$-depleted rats. Thus, immunofluorescence studies were performed to identify whether $\mathrm{NKCC} 1$ and $\mathrm{BK} \alpha$ channel specific proteins are localized in the basolateral and mucosal membranes of the same cell(s) in distal colon sections. As shown in Fig. 5, NKCC1-like proteins in normal distal colon were localized predominantly in the lateral membranes of lower crypt epithelia, with minimal labelling observed in surface cells (Fig. 5 A,D,I,J). BK $\alpha$-like proteins were diffusely localized in surface epithelial cells, with some apical membrane localization apparent within the crypt epithelium (Fig. 5 B,D,I,J). In contrast, NKCC1-like proteins are localized in the lateral membranes of both upper- and lower-crypt epithelia, as well as surface cells in $\mathrm{Na}^{+}$depleted rat distal colon (Fig. 5 E,H,K,L). In addition, BK $\alpha$-like proteins were concentrated on the mucosal membranes of both upper- and lower-crypt epithelia of dietary $\mathrm{Na}^{+}$-depleted rat distal colon (Fig. 5B), while remaining diffuse in surface epithelial cells. $\mathrm{BK} \alpha$, but not $\mathrm{NKCC} 1$ proteins were also localized in smooth muscle cells, as well as other unidentified cell types in the lamina propria of both normal and dietary $\mathrm{Na}^{+}$-depleted rat distal colon (Fig. 5 B,F). 
Merged images clearly indicate that $\mathrm{NKCC} 1$ and $\mathrm{BK} \alpha$-channel proteins are localized in basolateral and mucosal membranes, respectively, in epithelial cells of the crypts of normal and dietary $\mathrm{Na}^{+}$-depleted rat distal colon (Fig. 5 D,H,J,L). Qualitatively, it appears that both NKCC1 and BK-channel protein expression were substantially increased in dietary $\mathrm{Na}^{+}$-depleted rat distal colon, consistent with our qRT-PCR and Western blot data (Fig. 1). These observations further support the working hypothesis that the expression of basolateral NKCC1 and mucosal BKa channel proteins are coordinately regulated to produce the electrogenic $\mathrm{K}^{+}$secretion induced by aldosterone in the distal colon, and further suggest that the crypt region may be the primary location of aldosterone-induced $\mathrm{K}^{+}$secretion that is mediated by $\mathrm{NKCC} 1$ and $\mathrm{BK}$ channels.

Enhanced $\mathrm{NKCCl}$ expression does not support enhanced $\mathrm{Cl}^{-}$secretion in dietary $\mathrm{Na}^{+}$depleted rat distal colon.

Studies were next designed to determine whether in addition to functioning as a basolateral $\mathrm{K}^{+}$loader, increased $\mathrm{NKCC} 1$ expression would also function as $\mathrm{Cl}^{-}$loader supporting active $\mathrm{Cl}^{-}$ secretion in dietary $\mathrm{Na}^{+}$-depleted rat distal colon, as active $\mathrm{Cl}^{-}$secretion is known to be dependent on basolateral NKCC1 activity. ${ }^{39}{ }^{36} \mathrm{Cl}^{-}$flux experiments have previously demonstrated that both absorption and secretion of $\mathrm{Cl}^{-}$are reduced in $\mathrm{Na}^{+}$-depleted rat distal colon ${ }^{27}$, indicating that NKCC1 up-regulation may be unrelated to basal $\mathrm{Cl}^{-}$secretion. Our IsC data support this, as in the presence of mucosal $\mathrm{K}^{+}$channel blockers $\mathrm{Ba}^{2+}$ or IbTX, ISC remained negative (Fig. 4). However, aldosterone's regulation of stimulated $\mathrm{Cl}^{-}$secretion in the distal colon - and its dependence on NKCC1 - is not known. Thus, the effect of forskolin (FSK; an adenylyl cyclase activator that stimulated cAMP-dependent $\mathrm{Cl}^{-}$secretion) was assessed in normal and dietary $\mathrm{Na}^{+}$-depleted rat distal colon. 
When added to the serosal bath, FSK induced a robust increase in $\mathrm{I}_{\mathrm{SC}}$ (i.e., $\mathrm{Cl}^{-}$secretion) in both normal and dietary $\mathrm{Na}^{+}$-depleted rat distal colon (normal vs dietary $\mathrm{Na}^{+}$-depleted: $\Delta \mathrm{I}_{\mathrm{SC}(\mathrm{FSK})}$ $=123.6 \pm 15.1$ vs. $\left.92.4 \pm 22.8 \mu \mathrm{A} / \mathrm{cm}^{2} ; p=0.28\right)($ Fig. $6 \mathrm{~A}, \mathrm{C})$. The increased IsC was accompanied by increased $\mathrm{G}_{\mathrm{TE}}$ (normal vs dietary $\mathrm{Na}^{+}$-depleted: $\Delta \mathrm{G}_{\mathrm{TE}(\mathrm{FSK})}=4.2 \pm 0.6$ vs. $6.2 \pm 1.6 \mathrm{mS} / \mathrm{cm}^{2} ; p$ $=0.30)($ Fig. $6 \mathrm{~B}, \mathrm{D})$. Importantly, cAMP is also known to stimulate electrogenic $\mathrm{K}^{+}$secretion independently of $\mathrm{Cl}^{-}$secretion in normal distal colon ${ }^{40}$, which may ostensibly "mask" the FSKstimulated $\mathrm{Cl}^{-}$secretion under these conditions. However, when $\mathrm{Na}^{+}$-depleted rat distal colon was pre-treated for 60 minutes with mucosal $\mathrm{IbTX}$, neither $\Delta \mathrm{I}_{\mathrm{SC}(\mathrm{FSK})}\left(77.7 \pm 11.4 \mu \mathrm{A} / \mathrm{cm}^{2}\right)$ nor $\Delta \mathrm{G}_{\mathrm{TE}(\mathrm{FSK})}\left(4.0 \pm 0.6 \mathrm{mS} / \mathrm{cm}^{2}\right)$ were significantly different from either normal or untreated $\mathrm{Na}^{+}$depleted tissues ( $p>0.15$ for both) (Fig. 6A-D). Congruent with the finding that stimulated $\mathrm{Cl}^{-}$ secretion is not enhanced by aldosterone, abundance of cystic fibrosis transmembrane conductance regulator (CFTR; cAMP-activated $\mathrm{Cl}^{-}$channel) mRNA was also found to be unaffected by $\mathrm{Na}^{+}$ depletion (Fig. 6E). Thus, it is likely that the increased $\mathrm{NKCC1}$ expression induced by aldosterone primarily functions to support enhanced electrogenic $\mathrm{K}^{+}$secretion, without a concurrent increase in basal and/or stimulated $\mathrm{Cl}^{-}$secretion.

\section{DISCUSSION}

This study provides novel evidence that aldosterone increases the expression of both NKCC1 and BK channels in a coordinated manner to facilitate electrogenic $\mathrm{K}^{+}$, but not $\mathrm{Cl}^{-}$ secretion in rat distal colon. This conclusion is supported by the following observations: 1) both the activity (bumetanide-sensitive $\mathrm{I}_{\mathrm{SC}}$ ) and expression of $\mathrm{NKCC} 1$ (mRNA and protein) is enhanced by aldosterone, via dietary $\mathrm{Na}^{+}$depletion or by aldosterone application ex vivo, 2) $\mathrm{K}^{+}$secretion induced by aldosterone is mediated by basolateral $\mathrm{NKCC} 1$ and mucosal $\mathrm{BK}$ channels working in 
tandem, as indicated by the attenuating effect of mucosal IbTX on bumetanide-sensitive $\mathrm{I}_{\mathrm{SC}}$, as well as the apparent robust co-expression of $\mathrm{BK} \alpha$ and $\mathrm{NKCC} 1$ in crypt cells of $\mathrm{Na}^{+}$-depleted rats, and 3) aldosterone-induced upregulation of $\mathrm{NKCC} 1$ does not also facilitate an increase in basal or FSK-stimulated $\mathrm{Cl}^{-}$secretion, suggesting its regulation by aldosterone is specific to the $\mathrm{K}^{+}$ secretory pathway. Figure 8 summarizes these observations and proposes a basic cellular model of the regulation of $\mathrm{K}^{+}$secretion by aldosterone in the rat distal colon.

Stimulation of $\mathrm{K}^{+}$secretion is a fundamental effect of aldosterone signaling in the colonic epithelium and occurs independently of its parallel effects on electrogenic $\mathrm{Na}^{+}$absorption. ${ }^{24}$ While the effects of aldosterone on apical $\mathrm{K}^{+}$channels have been described in detail, here we provide evidence that expression of the basolateral $\mathrm{NKCC1}$ co-transporter is also under the control of aldosterone in the distal colon, which had not been demonstrated previously in rat. Further, we have shown that augmented $\mathrm{NKCC} 1$ activity contributes to the vectorial transport of $\mathrm{K}^{+}$, but not $\mathrm{Cl}^{-}$, across the colonic epithelium, suggesting a critical role for NKCC1 specifically in aldosteroneregulated $\mathrm{K}^{+}$transport.

Short-term aldosterone treatment has been recently shown to regulate $\mathrm{NKCC} 1$ in several epithelial and non-epithelial cell types (e.g. cochlear and smooth muscle) in vitro, ${ }^{25,41-43}$ indicating the phenomenon is not specific to intestinal epithelia. However, the role of NKCC1 in vectorial BK-mediated $\mathrm{K}^{+}$secretion had not been investigated. Moreover, the reported mechanism of NKCC1 up-regulation was suggested to be that aldosterone prevents its degradation via the proteasomal pathway, not via transcription upregulation, ${ }^{25,41}$ in contrast to our findings. This discrepancy may be due to inherent differences in the response to aldosterone between in native tissue and immortalized cell lines, or because of different environment factors associated with cell culture versus within native tissue. For example, short chain fatty acids produced by the 
microbiota, as well as neuronal input are both known to have a substantial impact on cellular homeostasis and electrolyte transport in vivo, ${ }^{44-48}$ but these factors are typically absent under normal cell culture conditions. Whether aldosterone also regulates the proteasomal degradation of $\mathrm{NKCC1}$ in native tissue remains to be determined but is entirely possible.

A somewhat controversial issue regarding colonic $\mathrm{K}^{+}$transport is whether crypt or surface epithelial regions (or both) participate in luminal $\mathrm{K}^{+}$secretion, ${ }^{10,34,38,49}$ and there may be considerable variation among species. Sorensen et. al. identified the presence of BK channels and BK-mediated $\mathrm{K}^{+}$secretion to be restricted to the crypt region in mice, and also found $\mathrm{NKCC} 1$ mRNA transcript to be elevated $\sim 2$-fold in the crypts of mice fed a high $\mathrm{K}^{+}$diet, another dietary model of hyperaldosteronism. ${ }^{10}$ Although obtained from rats and not mice, our $\mathrm{I}_{\mathrm{SC}}$ and immunofluorescence data are in agreement with these findings, as IbTX significantly attenuated the inhibition of $\mathrm{K}^{+}$secretion by bumetanide, while strong labelling of both $\mathrm{NKCC} 1$ and $\mathrm{BK} \alpha$ was apparent only in the crypts of $\mathrm{Na}^{+}$-depleted rat distal colonic sections. Jakob et. al. also demonstrated that $\mathrm{NKCC} 1$ expression is confined primarily to the crypt region throughout the rat GI tract. ${ }^{50}$ It is therefore likely that $\mathrm{NKCC} 1 / \mathrm{BK} \alpha$-mediated $\mathrm{K}^{+}$secretion, a process enhanced by aldosterone, occurs mainly in the crypt region of the distal colonic epithelium. However, aldosterone-induced $\mathrm{K}^{+}$secretion in surface epithelial cells has also been suggested to occur as a consequence of increased $\mathrm{ENaC}$ activity, favoring apical efflux of $\mathrm{K}^{+}$through one or more voltagegated $\mathrm{K}^{+}$channels in response to the depolarization brought on by $\mathrm{Na}^{+}$influx. ${ }^{51}$ Thus, luminal $\mathrm{K}^{+}$ secretion may occur in both surface and crypt regions simultaneously. Here, NKCC1 labelling was detected in surface epithelial cells of $\mathrm{Na}^{+}$-depleted animals, but clear membrane localization of BK channels was not apparent. Unfortunately, while $\mathrm{NKCC} 1 / \mathrm{BK}$-mediated $\mathrm{K}^{+}$secretion was clearly enhanced by aldosterone, the precise localization of this transport pathway within the tissue 
- whether solely in the crypt or the surface (or both) - cannot be determined with certainty based upon these experiments.

It should be noted that the degree of hyperaldosteronism induced by dietary $\mathrm{Na}^{+}$depletion was dependent on the sex of the animals. As described above, serum aldosterone was somewhat elevated in $\mathrm{Na}^{+}$-depleted females versus females fed a normal diet, but the effect of diet was much smaller in females compared to males. It is possible that sex hormone activity in some way modulates the response to aldosterone production or confers some resistance to the impact of $\mathrm{Na}^{+}$ depletion itself. Vivas et. al., using an acute model of $\mathrm{Na}^{+}$depletion by restricting dietary $\mathrm{Na}^{+}$and administering furosemide (a loop diuretic), found that male mice were more sensitive to the effects of $\mathrm{Na}^{+}$depletion in terms of resultant $\mathrm{Na}^{+}$appetite, as well as other cardiovascular and neuronal outcomes. ${ }^{52}$ However, serum aldosterone was not measured in these studies. While little work has been done to date, the mechanism(s) underlying the sex-specific effects of $\mathrm{Na}^{+}$depletion and accompanying hyperaldosteronism warrant further investigation, as this important aspect of mineralocorticoid function remains incompletely understood.

Alterations in colonic $\mathrm{K}^{+}$transport play an important role in various pathologies. ${ }^{1,53}$ For example, active colonic $\mathrm{K}^{+}$secretion via mucosal BK channels becomes imperative in patients with ESRD, providing an alternative outlet for $\mathrm{K}^{+}$and thus preventing life-threatening hyperkalemia. ${ }^{17,18}$ On the other hand, excessive $\mathrm{K}^{+}$secretion is a hallmark of colonic pseudoobstruction, contributing to diarrhea and hypokalemia in severe cases, ${ }^{13,14}$ with at least one documented case of a patient who was successfully treated with spironolactone, a mineralocorticoid antagonist, to reduce fecal $\mathrm{K}^{+}$excretion and hypokalemia associated with diarrhea. ${ }^{14}$ Another report demonstrated that spironolactone reduced deleterious fecal $\mathrm{K}^{+}$losses and significantly increased stool $\mathrm{Na}^{+}: \mathrm{K}^{+}$ratio in cholera patients, although overall stool rate was 
not affected. ${ }^{12}$ Accordingly, there is clinical value in furthering our understanding of mineralocorticoid regulation of colonic $\mathrm{K}^{+}$transport, as development of new interventions may prove useful in treating conditions like those described above.

In conclusion, here we provide evidence that aldosterone stimulates the expression of basolateral $\mathrm{NKCC1}$, supporting luminal $\mathrm{K}^{+}$, but not $\mathrm{Cl}^{-}$secretion in rat distal colon. Establishing that $\mathrm{NKCC} 1$ is an aldosterone-regulated component to the $\mathrm{K}^{+}$secretory pathway in the colonic epithelium may aid in the development of novel therapeutic strategies centered around the regulation of $\mathrm{K}^{+}$transport, such as colonic pseudo-obstruction or ESRD. 


\section{REFERENCES}

1. Sorensen M V., Matos JE, Praetorius HA, Leipziger J. Colonic potassium handling. Pflugers Arch Eur J Physiol. 2010;459(5):645-656. doi:10.1007/s00424-009-0781-9

2. Archampong EQ, Harris J, Clark CG. The absorption and secretion of water and electrolytes across the healthy and the diseased human colonic mucosa measured in vitro. 1972:880-886.

3. Foster S, Hayslett JP, Binder J, Emily S, Hayslew JP, Henry J. Mechanism of active potassium absorption and secretion in the rat colon. 1984.

4. Kliger AS, Binder HJ, Bastl C, Hayslett JP. Demonstration of active potassium transport in the mammalian colon. J Clin Invest. 1981;67(4):1189-1196. doi:10.1172/JCI110134

5. Rajendran VM, Sandle GI. Colonic Potassium Absorption and Secretion in Health and Disease. Compr Physiol. 2018;8(4):1513-1536. doi:10.1002/cphy.c170030

6. Rajendran VM, Singh SK, Geibel J, Binder HJ. Differential localization of colonic H+$\mathrm{K}+$-ATPase isoforms in surface and crypt cells. Am J Physiol - Gastrointest Liver Physiol. 1998;274(2 37-2):424-429. doi:10.1152/ajpgi.1998.274.2.g424

7. Sunuwar L, Asraf H, Donowitz M, Sekler I, Hershfinkel M. The Zn2+-sensing receptor, $\mathrm{ZnR} / \mathrm{GPR} 39$, upregulates colonocytic $\mathrm{Cl}-$ absorption, via basolateral $\mathrm{KCC} 1$, and reduces fluid loss. Biochim Biophys Acta - Biomembr. 2017;1863(4):947-960.

doi:10.1016/j.bbadis.2017.01.009.

8. Rehman S, Narayanan K, Nickerson AJ, et al. Parallel intermediate conductance K+ and $\mathrm{Cl}$ - channel activity mediates electroneutral $\mathrm{K}+$ exit across basolateral membranes in rat distal colon. Am J Physiol - Gastrointest Liver Physiol. 2020;319(2):G142-G150. doi:10.1152/ajpgi.00011.2020

9. Sausbier M, Matos JE, Sausbier U, et al. Distal colonic K+ secretion occurs via BK channels. J Am Soc Nephrol. 2006;17(5):1275-1282. doi:10.1681/ASN.2005101111

10. Sørensen M V., Strandsby AB, Larsen CK, Praetorius HA, Leipziger J. The secretory KCa1.1 channel localises to crypts of distal mouse colon: Functional and molecular evidence. Pflugers Arch Eur J Physiol. 2011;462(5):745-752. doi:10.1007/s00424-011$1000-\mathrm{z}$

11. Sweiry JH, Binder HJ. Characterization of aldosterone-induced potassium secretion in rat distal colon. J Clin Invest. 1989;83(3):844-851. doi:10.1172/JCI113967

12. Guerrant RL, Chen LC, Rohde JE. Effect of spironolactone on stool electrolyte losses during human cholera. Gut. 1972;13(3):197-200. doi:10.1136/gut.13.3.197 
13. Van Dinter TG, Fuerst FC, Richardson CT, et al. Stimulated active potassium secretion in a patient with colonic pseudo-obstruction: A new mechanism of secretory diarrhea. Gastroenterology. 2005;129(4):1268-1273. doi:10.1053/j.gastro.2005.07.029

14. Ram P, Goyal A, Lu M, Sloan J, McElhaugh W. Use of Aldosterone Antagonist to Treat Diarrhea and Hypokalemia of Ogilvie's Syndrome. Case Rep Gastrointest Med. 2016;2016:1-3. doi:10.1155/2016/1207240

15. Kanthesh BM, Sandle GI, Rajendran VM. Enhanced K+ secretion in dextran sulfateinduced colitis reflects upregulation of large conductance apical $\mathrm{K}+$ channels $(\mathrm{BK}$; Kcnma1). AJP Cell Physiol. 2013;305(9):C972-C980. doi:10.1152/ajpcell.00165.2013

16. Sandle GI, Perry MD, Mathialahan T, et al. Altered cryptal expression of luminal potassium (BK) channels in ulcerative colitis. J Pathol. 2007;212(1):66-73. doi:10.1002/path.2159

17. Mathialahan T, Sandle GI. Dietary potassium and laxatives as regulators of colonic potassium secretion in end-stage renal disease. Nephrol Dial Transplant. 2003;18(2):341347. doi:10.1093/ndt/18.2.341

18. Mathialahan T, Maclennan KA, Sandle LN, Verbeke C, Sandle GI. Enhanced large intestinal potassium permeability in end-stage renal disease. J Pathol. 2005;206(1):46-51. doi:10.1002/path.1750

19. Tumamian SG, Binder HJ. Regulation of active sodium and potassium transport in the distal colon of the rat. J Clin Invest. 1989;84(6):1924-1929.

20. Foster ES, Jones WJ, Hayslett JP, Binder HJ. Role of Aldosterone and Dietary Potassium in Potassium Adaptation in the Distal Colon of the Rat. Gastroenterology. 1985;88(1):4146. doi:10.1016/S0016-5085(85)80130-X

21. Amasheh S, Epple HJ, Mankertz J, et al. Differential Regulation of ENaC by Aldosterone in Rat Early and Late Distal Colon. Ann N Y Acad Sci. 2006;915(1):92-94. doi:https://doiorg.wvu.idm.oclc.org/10.1111/j.1749-6632.2000.tb05227.x

22. Greig ER, Baker EH, Mathialahan T, Boot-Handford RP, Sandle GI. Segmental variability of ENaC subunit expression in rat colon during dietary sodium depletion. Pflugers Arch Eur J Physiol. 2002;444(4):476-483. doi:10.1007/s00424-002-0828-7

23. Sørensen M V, Matos JE, Sausbier M, et al. Aldosterone increases KCa1.1 (BK) channelmediated colonic K+ secretion. J Physiol. 2008;586(Pt 17):4251-4264.

doi:10.1113/jphysiol.2008.156968

24. Rechkemmer G, Halm DANR. Aldosterone stimulates K secretion across mammalian colon independent of Na absorption. Proc Natl Acad Sci. 1989;86(January):397-401. 
25. Bazard P, Ding B, Chittam HK, et al. Aldosterone up-regulates voltage-gated potassium currents and NKCC1 protein membrane fractions. Sci Rep. 2020;10(1):1-14.

doi:10.1038/s41598-020-72450-4

26. Jiang C, Kawabe H, Rotin D. The ubiquitin ligase Nedd4L regulates the $\mathrm{Na} / \mathrm{K} / 2 \mathrm{Cl}$ cotransporter NKCC1/SLC12A2 in the colon. J Biol Chem. 2017;292(8):3137-3145. doi:10.1074/jbc.M116.770065

27. Halevy J, Budinger ME, Hayslett JP, Binder HJ. Role of Aldosterone in the Regulation of Sodium and Chloride Transport in the Distal Colon of Sodium-Depleted Rats. Gastroenterology. 1986;91(5):1227-1233. doi:https://doi.org/10.1016/S00165085(86)80021-X

28. Martin RS, Jones WJ, Hayslett JP. Animal model to study the effect of adrenal hormones on epithelial function. Kidney Int. 1983;24(3):386-391. doi:10.1038/ki.1983.171

29. $\mathrm{Hu} \mathrm{D}, \mathrm{Yu} \mathrm{ZL}$, Zhang Y, et al. Bumetanide treatment during early development rescues maternal separation-induced susceptibility to stress. Sci Rep. 2017;7(1):1-16. doi:10.1038/s41598-017-12183-z

30. Mhatre V. Ho and Kelsey C. Martin J-AL. Immunolocalization of the Ca2+-Activated K+ Channel Slo1 in Axons and Nerve Terminals of Mammalian Brain and Cultured Neurons. Bone. 2012;23(1):1-7. doi:10.1002/cne.20931.Immunolocalization

31. Clarke LL. A guide to Ussing chamber studies of mouse intestine. AJP Gastrointest Liver Physiol. 2009;296(6):G1151-G1166. doi:10.1152/ajpgi.90649.2008

32. Edmonds CJ, C.J. Edmonds C.L. Willis. THE EFFECT OF DIETARY SODIUM AND POTASSIUM INTAKE ON POTASSIUM SECRETION AND KINETICS IN RAT DISTAL COLON. J Physiol. 2019;53(9):1689-1699.

33. Hayslett JP, Myketey N, Binder HJ, Aronson PS. Mechanism of increased potassium secretion in potassium loading and sodium deprivation. Am J Physiol - Ren Fluid Electrolyte Physiol. 1980;8(4):378-382. doi:10.1152/ajprenal.1980.239.4.f378

34. Lomax B, Mcnicholas M, Lombes M, Sandle I, Mcnicholas M. Aldosterone-induced apical $\mathrm{Na}+$ and $\mathrm{K}+$ conductances are located primarily in surface cells in rat distal colon. 1994;29:G71-G82.

35. Escoubet B, Coureau C, Bonvalet JP, Farman N. Noncoordinate regulation of epithelial na channel and Na pump subunit mRNAs in kidney and colon by aldosterone. Am J Physiol Cell Physiol. 1997;272(5 41-5). doi:10.1152/ajpcell.1997.272.5.c1482

36. Hayslett JP, Binder HJ. Mechanism of potassium adaptation. Am J Physiol - Ren Fluid Electrolyte Physiol. 1982;12(2). doi:10.1152/ajprenal.1982.243.2.f103 
37. Singh SK, O’Hara B, Talukder JR, Rajendran VM. Aldosterone induces active K+ secretion by enhancing mucosal expression of Kcnn4c and Kcnmal channels in rat distal colon. Am J Physiol - Cell Physiol. 2012;302(9):1353-1360.

doi:10.1152/ajpcell.00216.2011

38. Halm ST, Liao T, Halm DR. Distinct K+ conductive pathways are required for Cl- and $\mathrm{K}+$ secretion across distal colonic epithelium. Am J Physiol - Cell Physiol. 2006;291(4):636648. doi:10.1152/ajpcell.00557.2005

39. Payne JA, Xu JC, Haas M, Lytle CY, Ward D, Forbush B 3rd. Primary structure, functional expression, and chromosomal localization of the bumetanide-sensitive Na-KCl cotransporter in human colon. J Biol Chem. 1995;270(30):17977-17985. doi:10.1074/jbc.270.30.17977

40. Sandle GI, Rajendran VM. Cyclic AMP-induced K+ secretion occurs independently of Clsecretion in rat distal colon. Am J Physiol - Cell Physiol. 2012;303(3):328-333. doi:10.1152/ajpcell.00099.2012

41. Ding B, Frisina RD, Zhu X, Sakai Y, Sokolowski B, Walton JP. Direct control of $\mathrm{Na}(+)-$ $\mathrm{K}(+)-2 \mathrm{Cl}(-)$-cotransport protein (NKCC1) expression with aldosterone. Am J Physiol Cell Physiol. 2014;306(1):C66-75. doi:10.1152/ajpcell.00096.2013

42. Tadros SF, Frisina ST, Mapes F, Frisina DR, Frisina RD. Higher serum aldosterone correlates with lower hearing thresholds: a possible protective hormone against presbycusis. Hear Res. 2005;209(1-2):10-18. doi:10.1016/j.heares.2005.05.009

43. Jiang G, Cobbs S, Klein JD, O'Neill WC. Aldosterone regulates the Na-K-2Cl cotransporter in vascular smooth muscle. Hypertens (Dallas, Tex 1979). 2003;41(5):1131-1135. doi:10.1161/01.HYP.0000066128.04083.CA

44. Gross ER, Gershon MD, Margolis KG, Gertsberg Z V., Cowles RA. Neuronal serotonin regulates growth of the intestinal mucosa in mice. Gastroenterology. 2012;143(2):408417.e2. doi:10.1053/j.gastro.2012.05.007

45. Inagaki A, Hayashi M, Andharia N, Matsuda H. Involvement of butyrate in electrogenic $\mathrm{K}$ + secretion in rat rectal colon. Pflugers Arch Eur J Physiol. 2019;471(2):313-327. doi:10.1007/s00424-018-2208-y

46. Cooke HJ. Role of the "little brain" in the gut in water and electrolyte homeostasis 1 . FASEB J. 1989;3(2):127-138. doi:10.1096/fasebj.3.2.2464517

47. Neunlist M, Toumi F, Oreschkova T, et al. Human ENS regulates the intestinal epithelial barrier permeability and a tight junction-associated protein ZO-1 via VIPergic pathways. Am J Physiol - Gastrointest Liver Physiol. 2003;285(5 48-5):1028-1036. doi:10.1152/ajpgi.00066.2003 
48. Cuff MA, Shirazi-Beechey SP. The importance of butyrate transport to the regulation of gene expression in the colonic epithelium. Biochem Soc Trans. 2004;32(6):1100-1102. doi:10.1042/BST0321100

49. Hay-Schmidt A, Grunnet M, Abrahamse SL, Knaus HG, Klaerke DA. Localization of $\mathrm{Ca} 2+$-activated big-conductance $\mathrm{K}+$ channels in rabbit distal colon. Pflugers Arch Eur J Physiol. 2003;446(1):61-68. doi:10.1007/s00424-002-0983-x

50. Jakab RL, Collaco AM, Ameen NA. Physiological relevance of cell-specific distribution patterns of CFTR, NKCC1, NBCe1, and NHE3 along the crypt-villus axis in the intestine. Am J Physiol - Gastrointest Liver Physiol. 2011;300(1):82-98. doi:10.1152/ajpgi.00245.2010

51. Grotjohann I, Gitter AH, Köckerling A, Bertog M, Schulzke JD, Fromm M. Localization of cAMP- and aldosterone-induced $\mathrm{K}+$ secretion in rat distal colon by conductance scanning. J Physiol. 1998;507(2):561-570. doi:10.1111/j.1469-7793.1998.561bt.x

52. Vivas L, Dadam FM, Caeiro XE. Sex differences in body fluid homeostasis: Sex chromosome complement influences on bradycardic baroreflex response and sodium depletion induced neural activity. Physiol Behav. 2015;152(Pt B):416-421. doi:10.1016/j.physbeh.2015.08.010

53. Heitzmann D, Warth R. Physiology and pathophysiology of potassium channels in gastrointestinal epithelia. Physiol Rev. 2008;88(3):1119-1182. doi:10.1152/physrev.00020.2007 


\section{FIGURES AND LEGENDS}

A

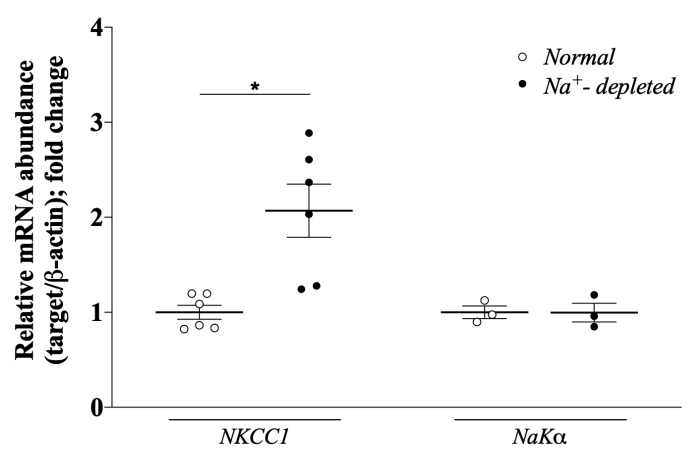

C

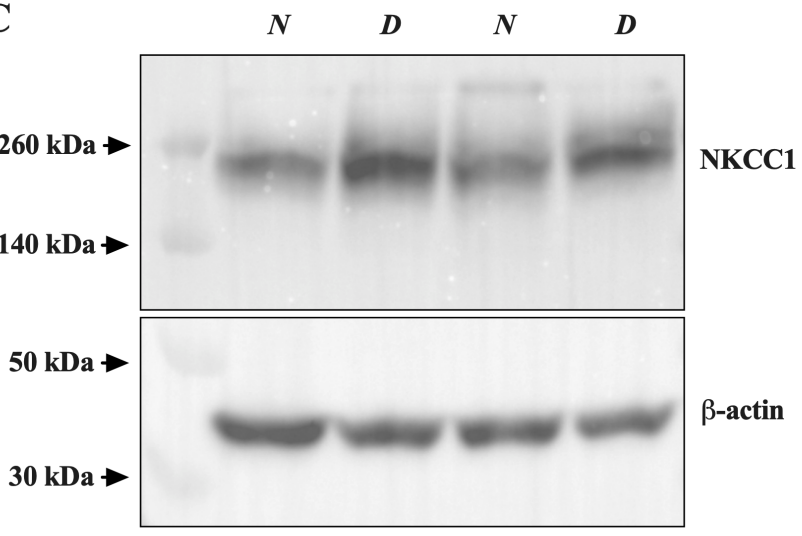

B

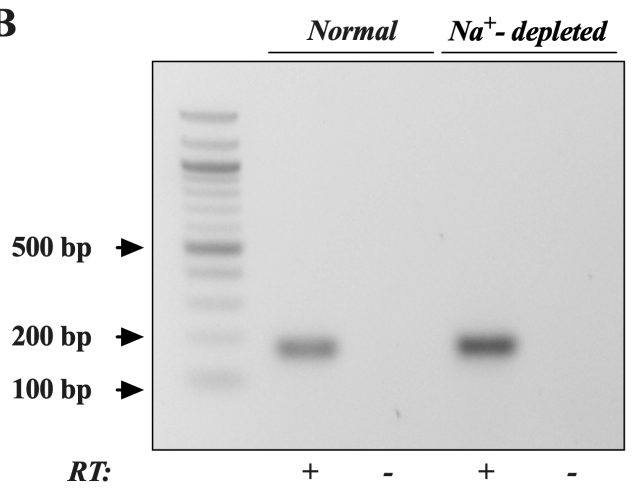

D

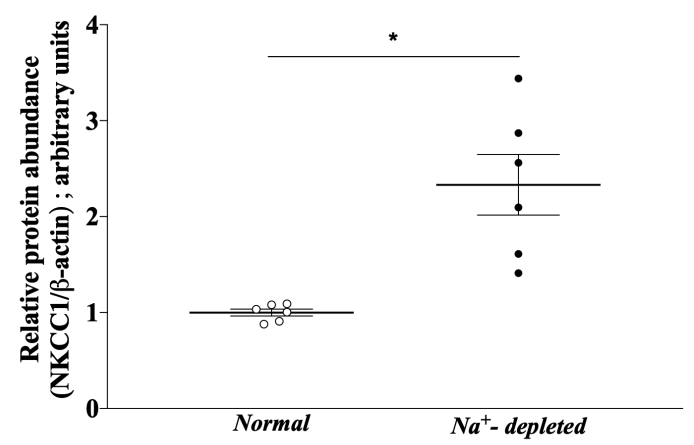

Figure 1. Dietary $\mathrm{Na}^{+}$depletion induces NKCC1 expression in rat distal colon. (A) NKCC1 and $\mathrm{Na}^{+}, \mathrm{K}^{+}$-ATPase $(\mathrm{NaK} \alpha)$ mRNA abundance measured by qRT-PCR using total-RNA isolated from normal and dietary- $\mathrm{Na}^{+}$depleted rat distal colon. (B) qRT-PCR-amplified NKCC1 transcript products were resolved on $1.5 \%$ agarose gel show a single product of expected size $(177 \mathrm{bp})$ in total-RNA isolated from both normal and dietary $\mathrm{Na}^{+}$-depleted rat distal colon. DNA ladder and corresponding fragment sizes are indicated on the left. (C) Representative Western blot of NKCC1 and $\beta$-actin (internal control) protein expression in mucosal homogenates from normal $(N)$ and dietary $\mathrm{Na}^{+}$-depleted $(D)$ rat distal colon. Protein ladder is shown in the extreme left lane, and corresponding molecular weights in $\mathrm{kDa}$ are indicated. (D) Densitometry quantitation of NKCC1 
protein normalized to $\beta$-actin. NKCC1 band intensity was measured and divided by the $\beta$-actin band intensity for each sample using FIJI (ImageJ) software. Data were then transformed so that control NKCC1/ $\beta$-actin values were set equal to 1 . Lines and error bars represent means $\pm \mathrm{SEM}$, ${ }^{*} p<0.01$, as determined by Student's unpaired t-test. 
A

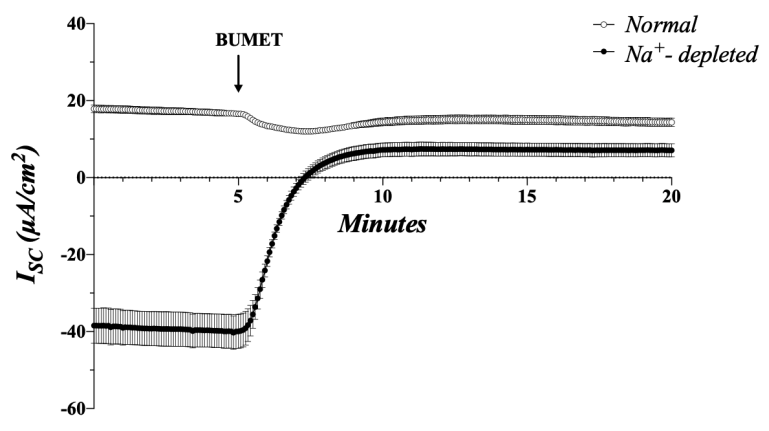

C

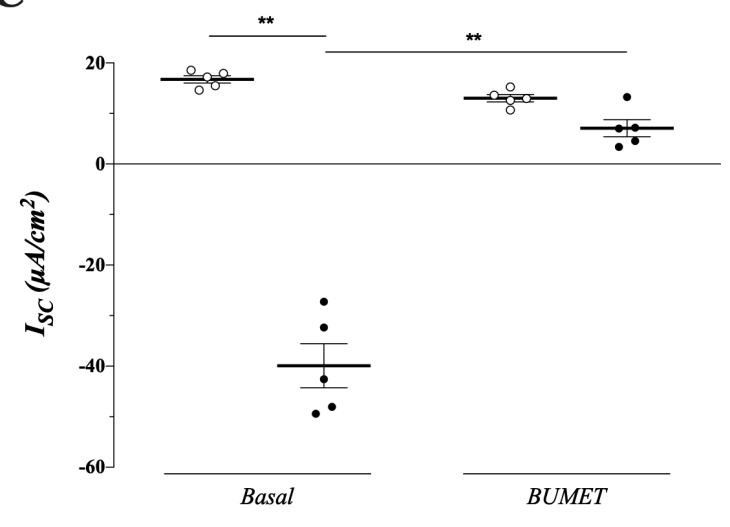

$\mathbf{E}$

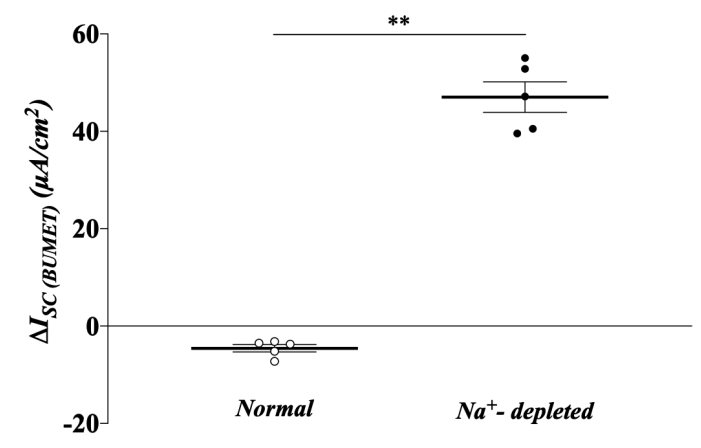

B

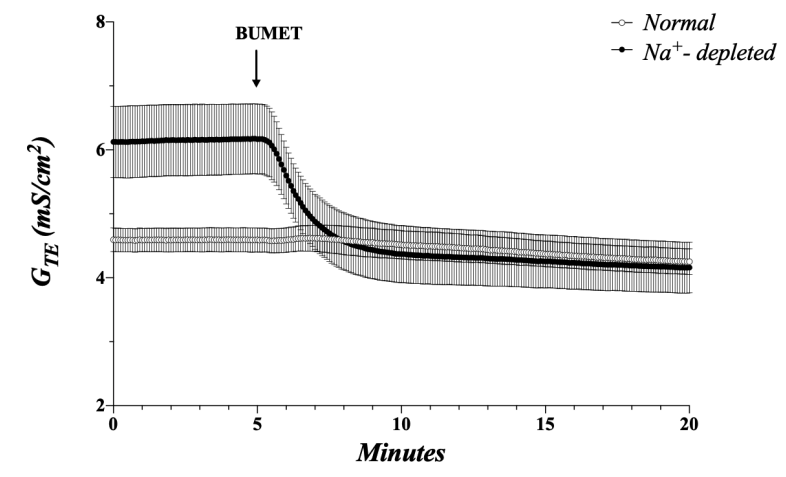

D

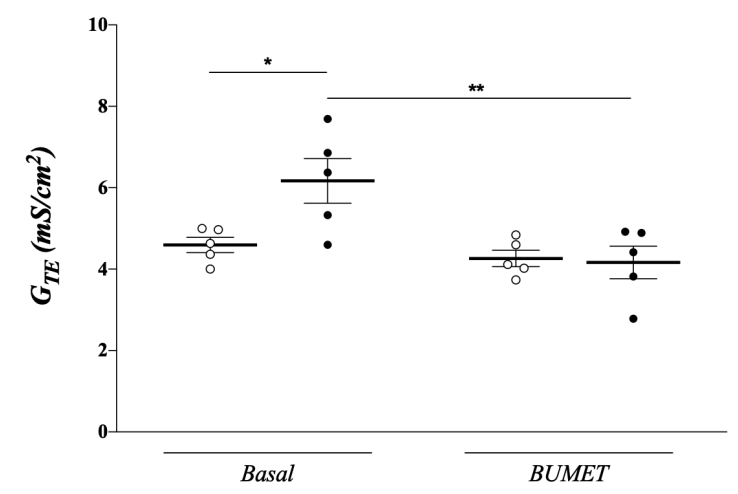

$\mathbf{F}$

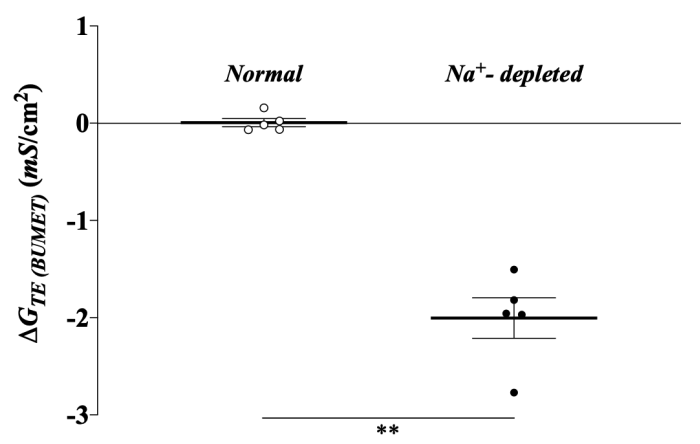

Figure 2. Inhibition of NKCC1 significantly inhibits electrogenic $\mathrm{K}^{+}$secretion in dietary $\mathrm{Na}^{+}-$ depleted, but not in normal rat distal colon. $\mathrm{I}_{\mathrm{SC}}(\mathrm{A})$ and $\mathrm{G}_{\mathrm{TE}}(\mathrm{B})$ measurements from normal 
(open circles) and dietary $\mathrm{Na}^{+}$-depleted (closed circles) distal colonic mucosae in the presence of $100 \mu \mathrm{M}$ amiloride in the mucosal bath. Tissues were treated with $200 \mu \mathrm{M}$ bumetanide (BUMET) in the serosal bath where indicated. (C-D) Group data showing $\mathrm{I}_{\mathrm{SC}}(\mathrm{C})$ and $\mathrm{G}_{\mathrm{TE}}(\mathrm{D})$ from normal and aldo tissues are before and after application of bumetanide, in the presence of mucosal amiloride. Average $\mathrm{I}_{\mathrm{SC}}$ and $\mathrm{G}_{\mathrm{TE}}$ values over the course of 1 minute immediately prior to bumetanide addition (basal), and 1 minute at the end of the recording period (BUMET) were calculated for each tissue. (E-F) Change in $(\Delta) \mathrm{I}_{\mathrm{SC}}(\mathrm{E})$ and $\mathrm{G}_{\mathrm{TE}}(\mathrm{F})$ induced by bumetanide in normal and dietary $\mathrm{Na}^{+}$-depleted rat distal colon in the presence of mucosal amiloride. $\Delta \mathrm{I}_{\mathrm{SC}}$ and $\Delta \mathrm{G}_{\mathrm{TE}}$ were calculated for each tissue by subtracting the basal value from the value at the end of the recording period, after BUMET addition. Lines and error bars represent means \pm SEM. * $p<0.05,{ }^{* *} p<0.01$ as determined by one-way ANOVA with Tukey's post-hoc (panels C-D) or Student's unpaired t-test (panels E-F). 
A

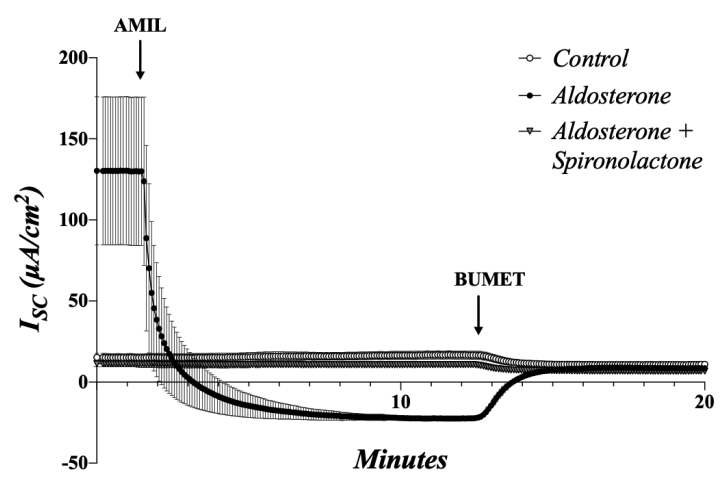

C

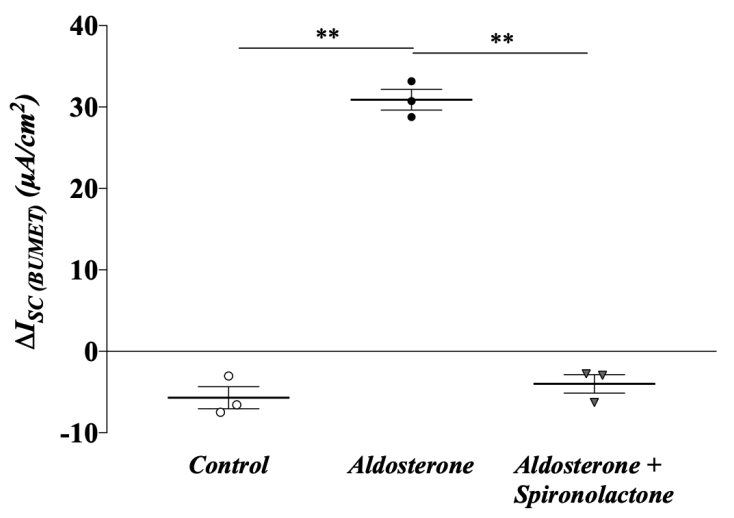

E

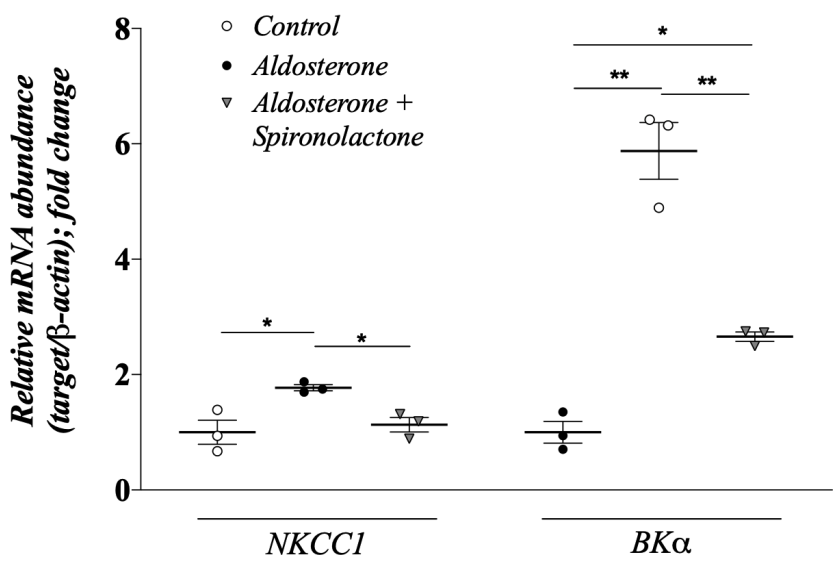

B

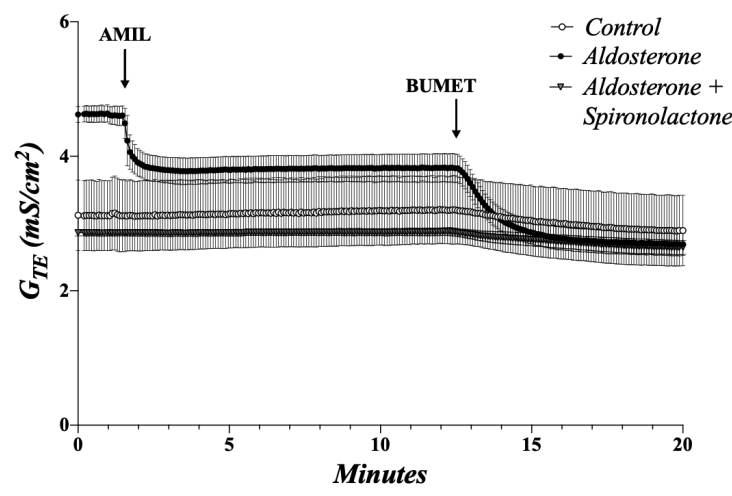

D

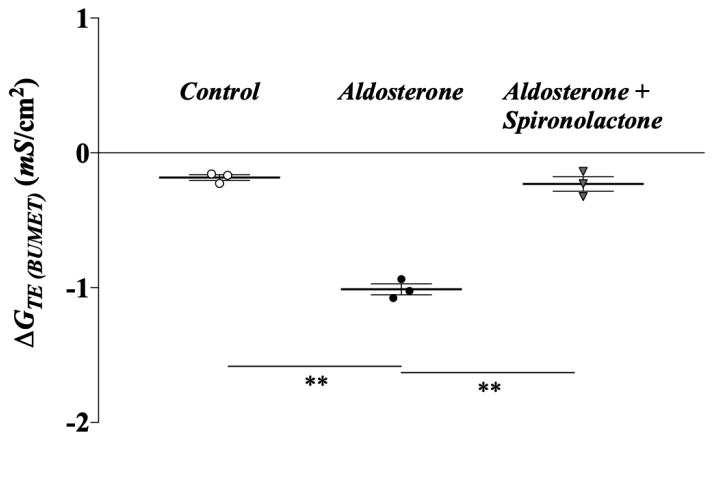


Figure 3. Ex vivo aldosterone induces $\mathrm{ENaC}$ activity and bumetanide-sensitive $\mathrm{K}^{+}$secretion and increases $\mathrm{NKCC1}$ and $\mathrm{BK} \alpha$ mRNA abundance in normal rat distal colon. $\mathrm{I}_{\mathrm{SC}}(\mathrm{A})$ and transepithelial resistance $\left(\mathrm{G}_{\mathrm{TE}}\right.$; B) were continuously monitored in normal rat distal colonic mucosae mounted under voltage clamp conditions in the presence and absence of $10 \mathrm{nM}$ aldosterone, or $10 \mathrm{nM}$ aldosterone plus $10 \mu \mathrm{M}$ spironolactone. Following an 8-hour incubation period, $\mathrm{I}_{\mathrm{SC}}$ and $\mathrm{G}_{\mathrm{TE}}$ were progressively increased (evidence for induced ENaC activity) in aldosterone-treated tissues (closed circles), while no change was observed in control (open circles) or aldosterone plus spironolactone-treated tissues (grey triangles). $\mathrm{I}_{\mathrm{SC}}$ and $\mathrm{G}_{\mathrm{TE}}$ data over the 8-hour incubation period are not shown here, so as to prevent the depicted data from being distorted by the lengthy time scale. $\mathrm{I}_{\mathrm{SC}}$ recordings depicted here were begun at 8 hours post-treatment. All tissues were treated with mucosal amiloride (AMIL; $100 \mu \mathrm{M}$ ), followed by serosal bumetanide (BUMET; $200 \mu \mathrm{M}$ ) where indicated. (C-D) Group data showing the bumetanide-sensitive change in Isc $\left(\Delta \mathrm{I}_{\mathrm{SC}} ; \mathrm{C}\right)$ and $\mathrm{G}_{\mathrm{TE}}\left(\Delta \mathrm{G}_{\mathrm{TE}} ; \mathrm{D}\right)$ in control, aldosterone-, and aldosterone plus spironolactonetreated tissues. Average $\mathrm{I}_{\mathrm{SC}}$ and $\mathrm{G}_{\mathrm{TE}}$ values over the course of 1 minute immediately prior to bumetanide addition, and 1 minute at the end of the recording period were calculated for each tissue. $\Delta \mathrm{I}_{\mathrm{SC}}$ and $\Delta \mathrm{G}_{\mathrm{TE}}$ were calculated by subtracting the basal value from the value at the end of the recording period, after bumetanide application. (E) NKCC1 and BK $\alpha$ mRNA transcript abundance measured by qRT-PCR analysis using RNA extracted from control, aldosterone- and aldosterone plus spironolactone-treated mucosae used in the above experiments. Lines and error bars represent means \pm SEM. ${ }^{*} p<0.05$ and ${ }^{* *} p<0.01$, as determined by one-way ANOVA with Tukey's post-hoc. 
A

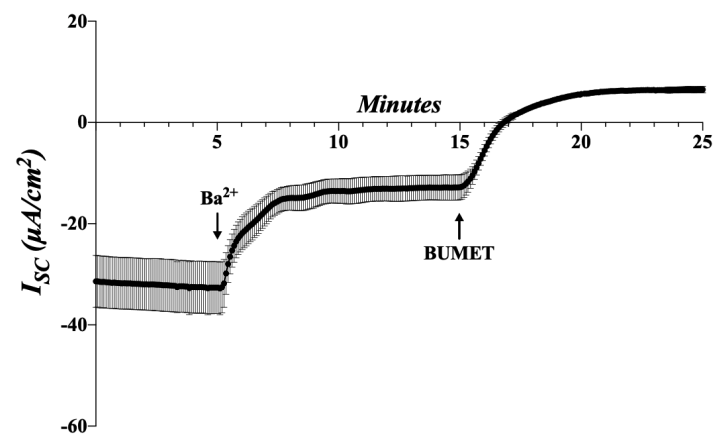

$\mathbf{C}$

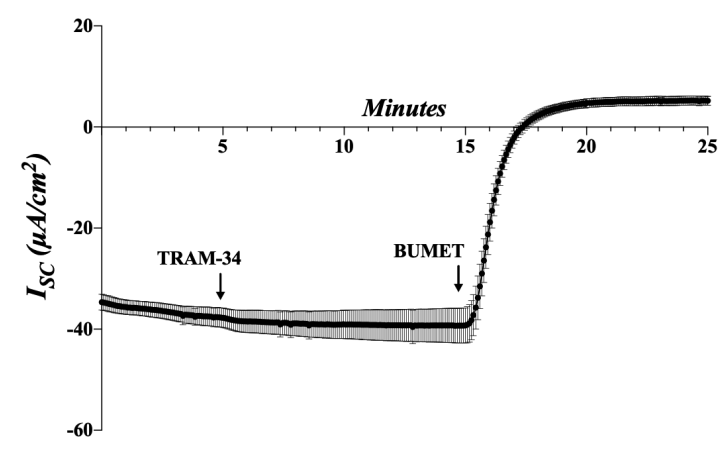

$\mathbf{E}$

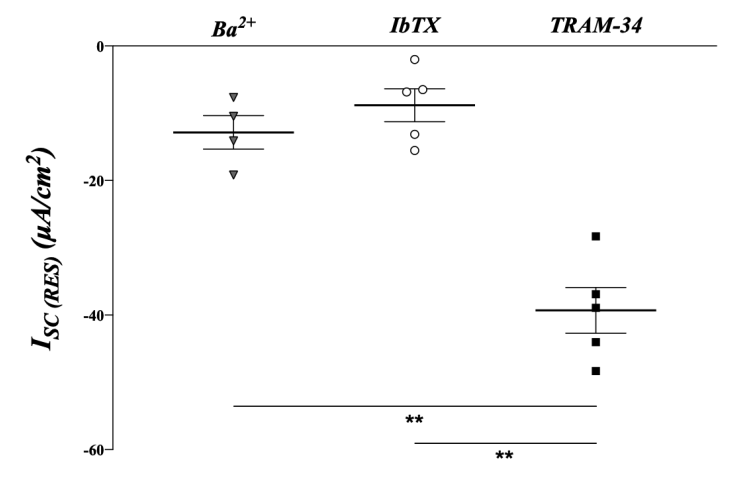

B

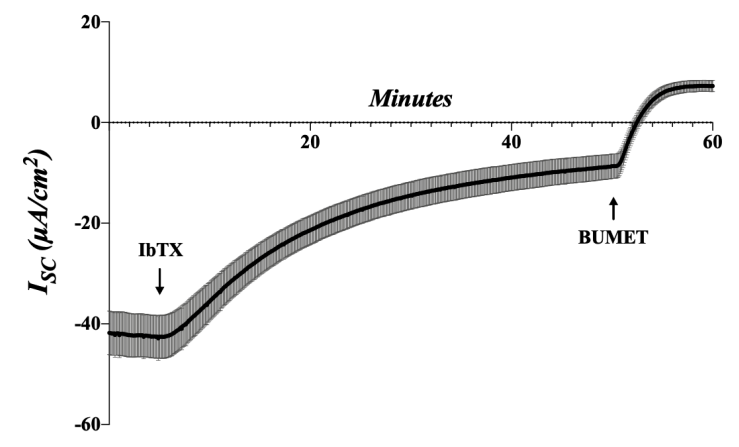

D

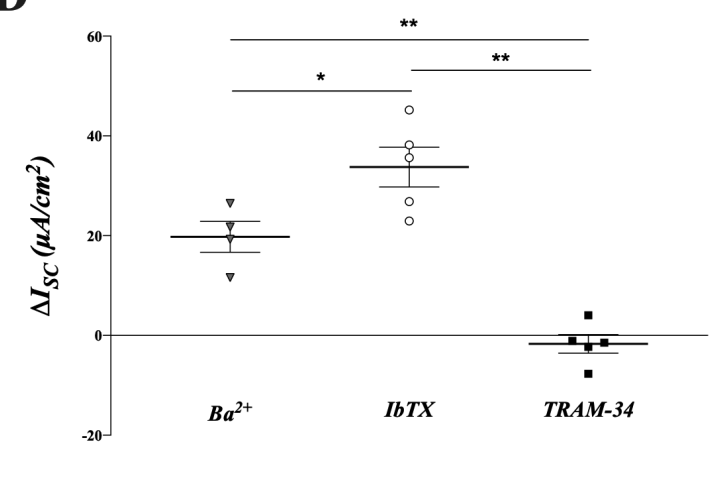

$\mathbf{F}$

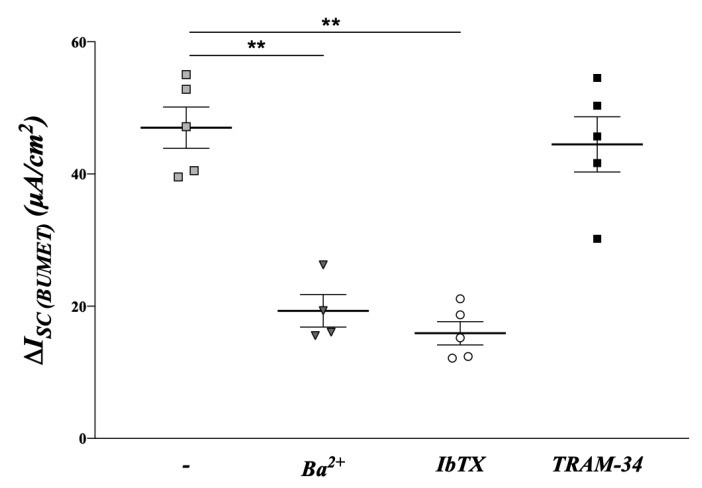

Figure 4. Mucosal $\mathrm{K}^{+}$channel blockers inhibit basal and bumetanide-sensitive electrogenic $\mathrm{K}^{+}$secretion in dietary $\mathrm{Na}^{+}$-depleted rat distal colon. $\mathrm{I}_{\mathrm{SC}}$ recordings from $\mathrm{Na}^{+}$-depleted rat distal colonic mucosae treated with mucosal $\mathrm{Ba}^{2+}(3 \mathrm{mM})(\mathrm{A})$, Iberiotoxin (IbTX; $\left.250 \mathrm{nM}\right)(\mathrm{B})$, 
or TRAM-34 (250 nM) (C) in the presence of $100 \mu \mathrm{M}$ mucosal amiloride. Tissues were subsequently treated with serosal bumetanide (BUMET; $200 \mu \mathrm{M})$. (D-E) Group data showing change in $\mathrm{I}_{\mathrm{SC}}\left(\Delta \mathrm{I}_{\mathrm{SC}} ; \mathrm{D}\right)$ in the presence of $\mathrm{Ba}^{2+}$, IbTX or TRAM-34, as well as the residual $\mathrm{I}_{\mathrm{SC}}$ (ISC(RES); E) after the application of each drug. Average $\mathrm{I}_{\mathrm{SC}}$ values over the course of 1 minute immediately prior to $\mathrm{Ba}^{2+}$, IbTX or TRAM-34 application, 1 minute immediately prior to the addition of bumetanide, and 1 minute at the end of the recording period were calculated for each tissue. (F) Change in $\mathrm{I}_{\mathrm{SC}}$ induced by bumetanide $\left(\Delta \mathrm{I}_{\mathrm{SC}(\mathrm{BUMET})}\right)$ in the presence IbTX, Tram-34 or $\mathrm{Ba}^{2+} . \Delta \mathrm{I}_{\mathrm{SC}}$ values were calculated by subtracting residual $\mathrm{I}_{\mathrm{SC}}$ after Ba2+, IbTX or TRAM-34 application, from the final $\mathrm{I}_{\mathrm{SC}}$ measured after bumetanide application. Data points with no inhibitor (-) (far left) are re-plotted from Fig. 4E for comparison. Lines and error bars represent means \pm SEM. ${ }^{*} p<0.05$ and ${ }^{* *} p<0.01$, as determined by one-way ANOVA with Tukey’s posthoc. 


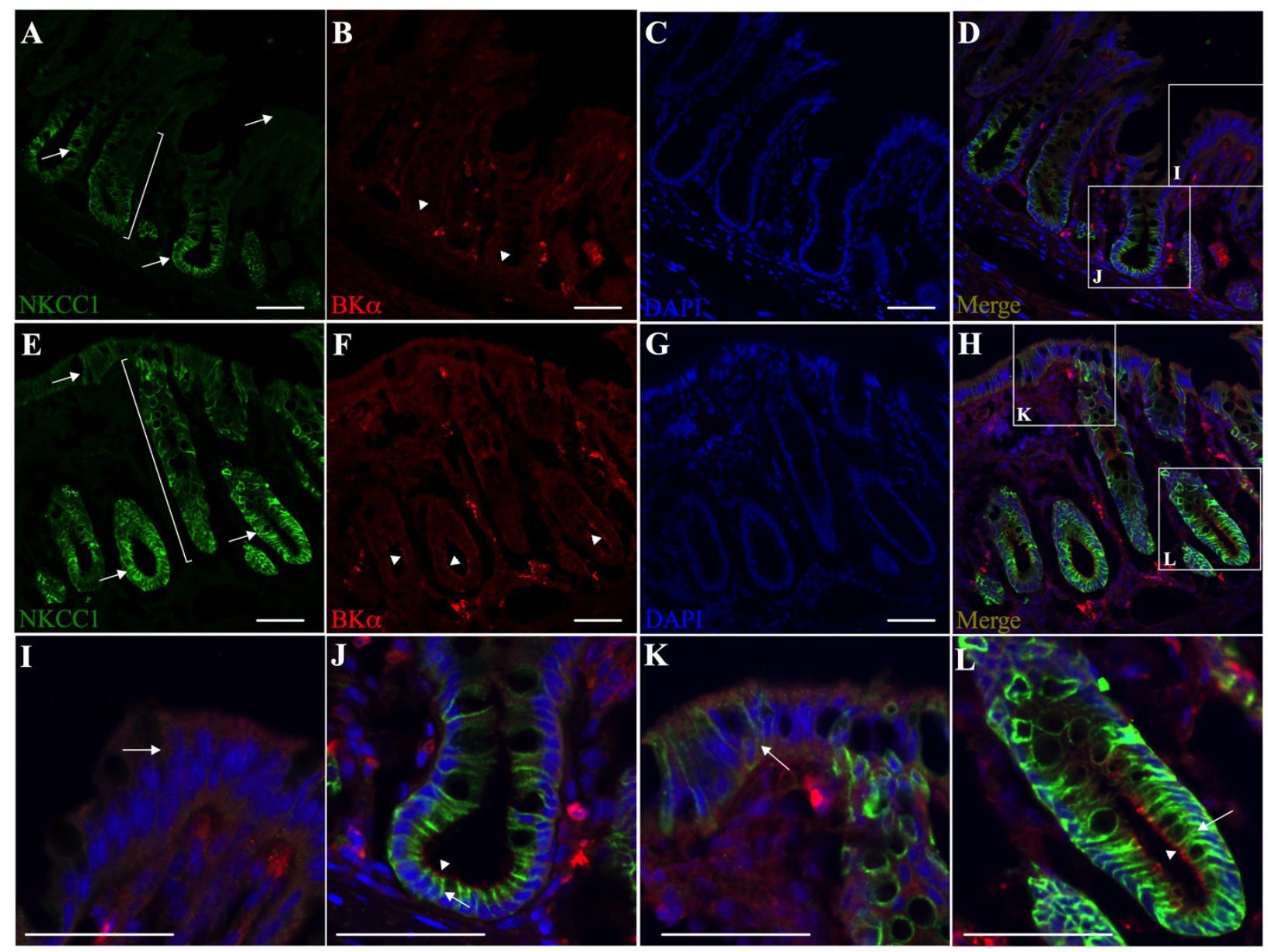

Figure 5. Immunofluorescence localization of $\mathrm{NKCC1}$ and $\mathrm{BK} \alpha$ channel specific proteins in normal and dietary $\mathrm{Na}^{+}$-depleted rat distal colon. Normal (A-D) and dietary $\mathrm{Na}^{+}$-depleted (EH) rat distal colon sections were labelled with both anti-NKCC1 (green) and anti-BKa (red) antibodies, while nuclei were labelled with DAPI (blue). Merged images are shown in panels D and $\mathrm{H}$. All images were captured at 20x magnification, scale bar $=50 \mu \mathrm{m}$. Magnified surface cell regions are shown for normal (I) and dietary $\mathrm{Na}^{+}$-depleted $(\mathrm{K})$ rat distal colon images, as indicated by the white boxes in panels D and H. Magnified crypt regions are also shown for normal (J) and dietary $\mathrm{Na}^{+}$-depleted (L) rat distal colon. Arrows indicate basolateral membrane labelling of NKCC1 in the lower crypt region of normal (A,J), and the whole crypt as well as surface cells in $\mathrm{Na}^{+}$-depleted rat distal colon sections $(\mathrm{E}, \mathrm{K}, \mathrm{L})$. Brackets in panels $\mathrm{A}$ and $\mathrm{E}$ illustrate that NKCC1 
labelling appears mostly restricted to the lower crypt in normal, while extending upward to the surface region in $\mathrm{Na}^{+}$-depleted rat distal colon. Arrowheads indicate the apparent mucosal membrane-localized BK channels in the crypts of both normal $(\mathrm{B}, \mathrm{J})$ and $\mathrm{Na}^{+}$-depleted $(\mathrm{F}, \mathrm{L})$ rat distal colon sections, while BK labelling in the surface regions was diffuse in both normal and $\mathrm{Na}^{+}$-depleted colon sections.

A

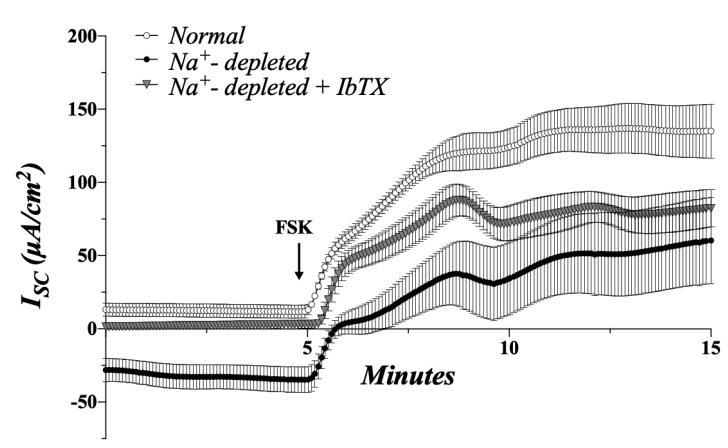

$\mathbf{C}$

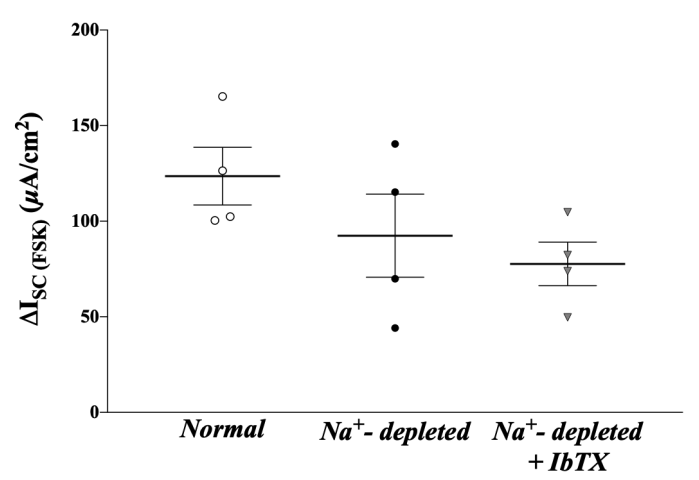

$\mathbf{E}$

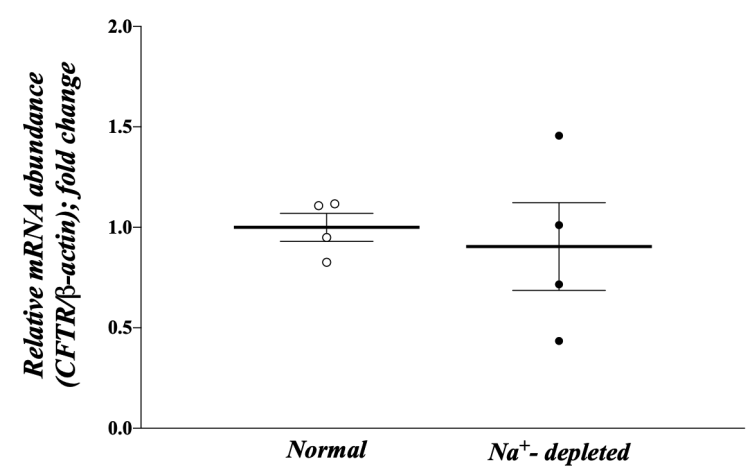

B

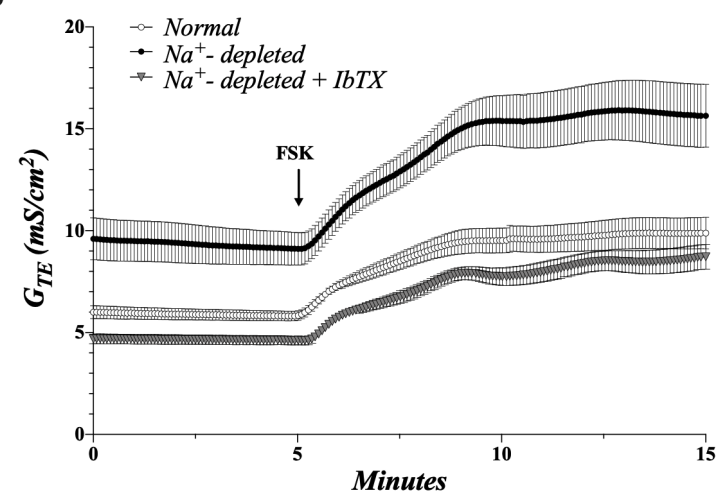

D

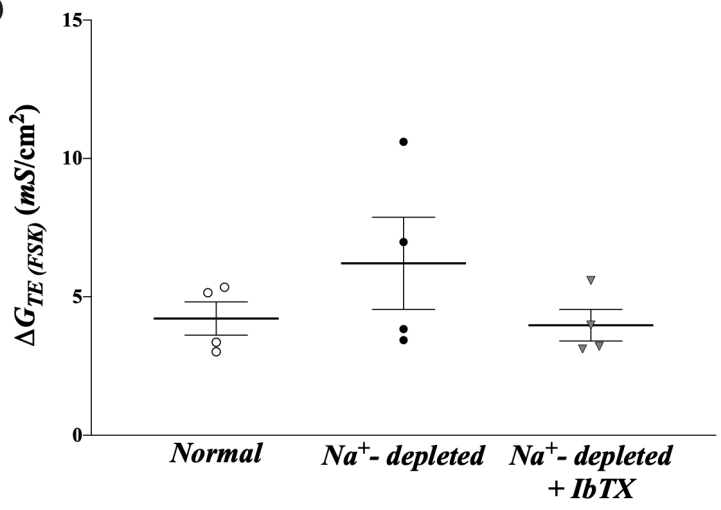


Figure 6. Enhanced NKCC1 expression does not support increased capacity for stimulated $\mathrm{Cl}^{-}$secretion. $\mathrm{I}_{\mathrm{SC}}(\mathrm{A})$ and $\mathrm{G}_{\mathrm{TE}}(\mathrm{B})$ measurements from normal (open circles) and dietary $\mathrm{Na}^{+}$ depleted (closed circles) rat distal colon, as well as dietary $\mathrm{Na}^{+}$-depleted rat distal colon pre-treated with mucosal IbTX $(250 \mathrm{nM})$ (grey triangles) in the presence of mucosal $100 \mu \mathrm{M}$ amiloride. Tissues were treated with $10 \mu \mathrm{M}$ forskolin (FSK) in the serosal bath where indicated. (C-D) Group data showing change in $(\Delta) \mathrm{I}_{\mathrm{SC}}(\mathrm{C})$ and $\mathrm{G}_{\mathrm{TE}}(\mathrm{D})$ induced by FSK in each group. Average $\mathrm{I}_{\mathrm{SC}}$ and $\mathrm{G}_{\mathrm{TE}}$ values over the course of 1 minute immediately prior to FSK addition, and 1 minute at the end of the recording period were calculated for each tissue. $\Delta \mathrm{I}_{\mathrm{SC}}$ and $\Delta \mathrm{G}_{\mathrm{TE}}$ were calculated by subtracting the basal value from the value at the end of the recording period, after FSK addition. Lines and error bars represent means \pm SEM.
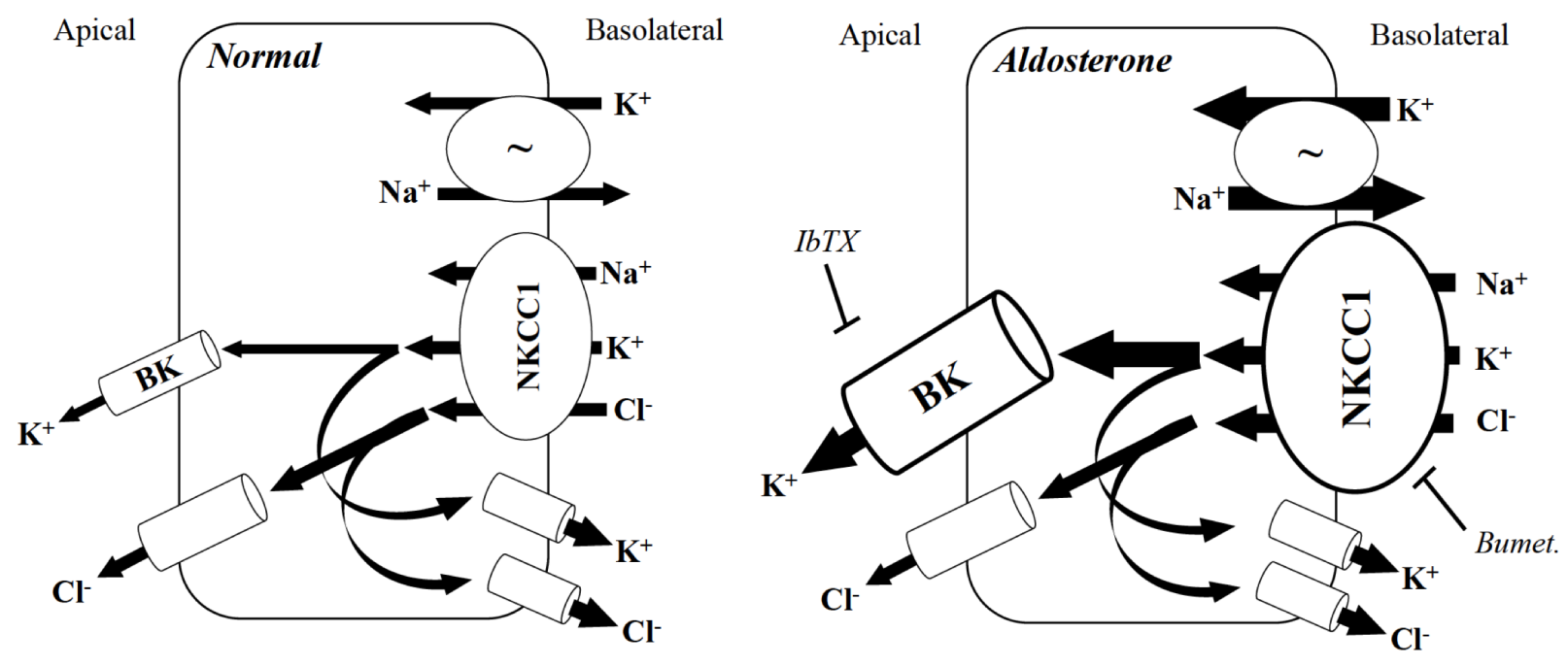

Figure 7. Cellular model of aldosterone-regulated electrogenic $\mathrm{K}^{+}$secretion in the distal colon. Normal distal colon secretes both $\mathrm{K}^{+}$and $\mathrm{Cl}^{-}$across the apical membrane. $\mathrm{Na}^{+}, \mathrm{K}^{+}$ATPase $(\sim)$ activity drives overall transport by establishing the $\mathrm{Na}^{+}$gradient that allows basolateral uptake 
of $\mathrm{K}^{+}$and $\mathrm{Cl}^{-}$via $\mathrm{NKCC1}$. Apical membrane secretion of $\mathrm{K}^{+}$and $\mathrm{Cl}^{-}$is supported by basolateral exit of $\mathrm{Cl}^{-}$and $\mathrm{K}^{+}$ions, respectively, to maintain the necessary electrochemical gradient. Multiple basolateral $\mathrm{K}^{+}$and $\mathrm{Cl}^{-}$channels may be involved in this process, including intermediate conductance $(\mathrm{IK})$ and voltage-gated $\left(\mathrm{K}_{\mathrm{V}} 7.1\right) \mathrm{K}^{+}$channels, as well as CLC-2 $\mathrm{Cl}^{-}$channels. Aldosterone stimulates electrogenic $\mathrm{K}^{+}$secretion by increasing basolateral $\mathrm{Na}^{+}, \mathrm{K}^{+}$ATPase activity (represented by enlarged arrows) and increasing the expression and activity of BK channels and NKCC1 co-transporter (represented by enlarged image objects and arrows, respectively). Electrogenic $\mathrm{K}^{+}$secretion is inhibited by either IbTX (apical membrane BK channels) or bumetanide (basolateral NKCC1). To support the aldosterone-induced sustained $\mathrm{K}^{+}$ secretion at the apical membrane, basolateral $\mathrm{Cl}^{-}$exit also must presumably be enhanced, although the identity of the channel(s) involved remains undetermined. Despite the increase in NKCC1 expression and activity, electrogenic $\mathrm{Cl}^{-}$secretion is not enhanced by aldosterone, suggesting that the regulation of NKCC1 by aldosterone is specific to the $\mathrm{K}^{+}$transport pathway. 


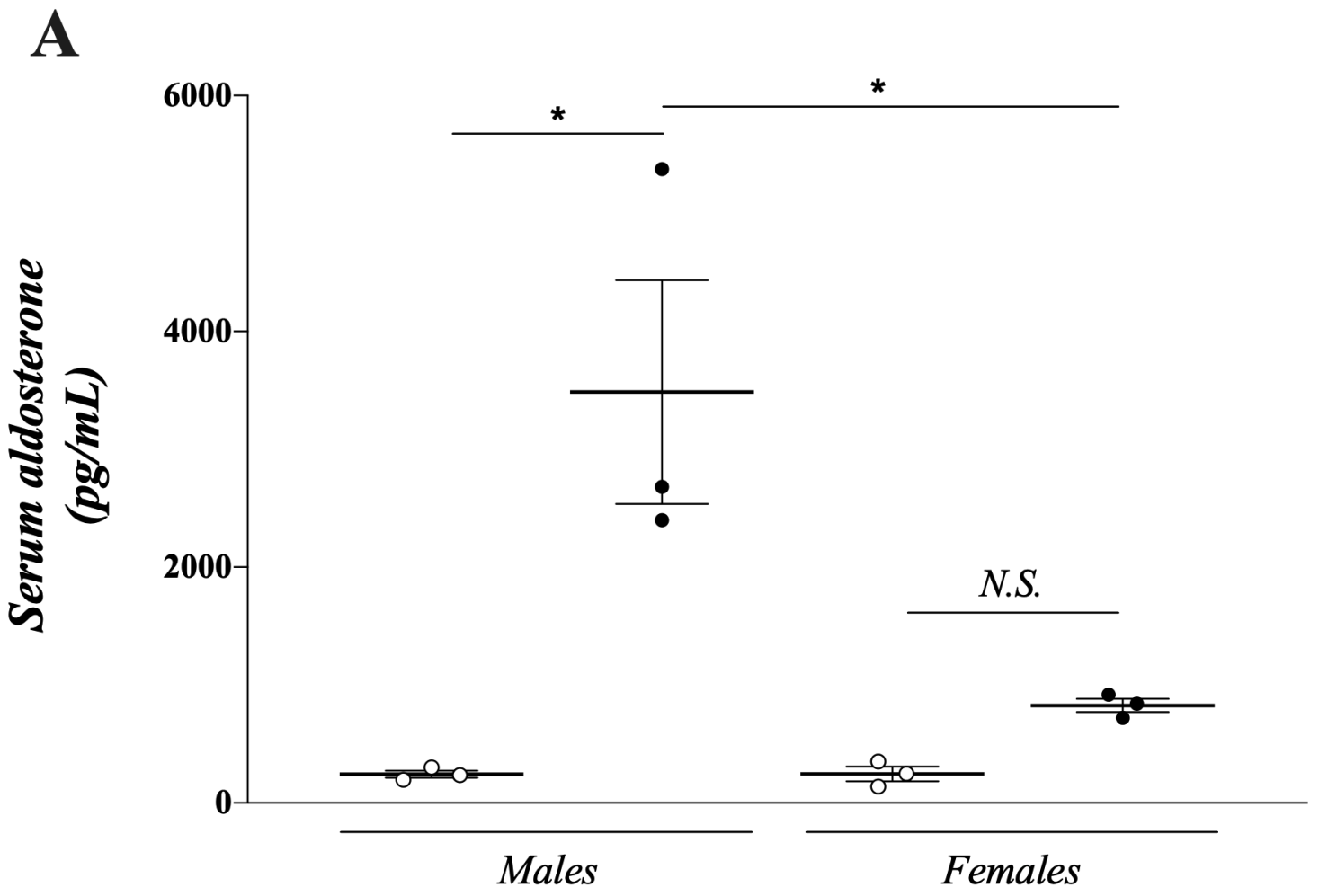

B

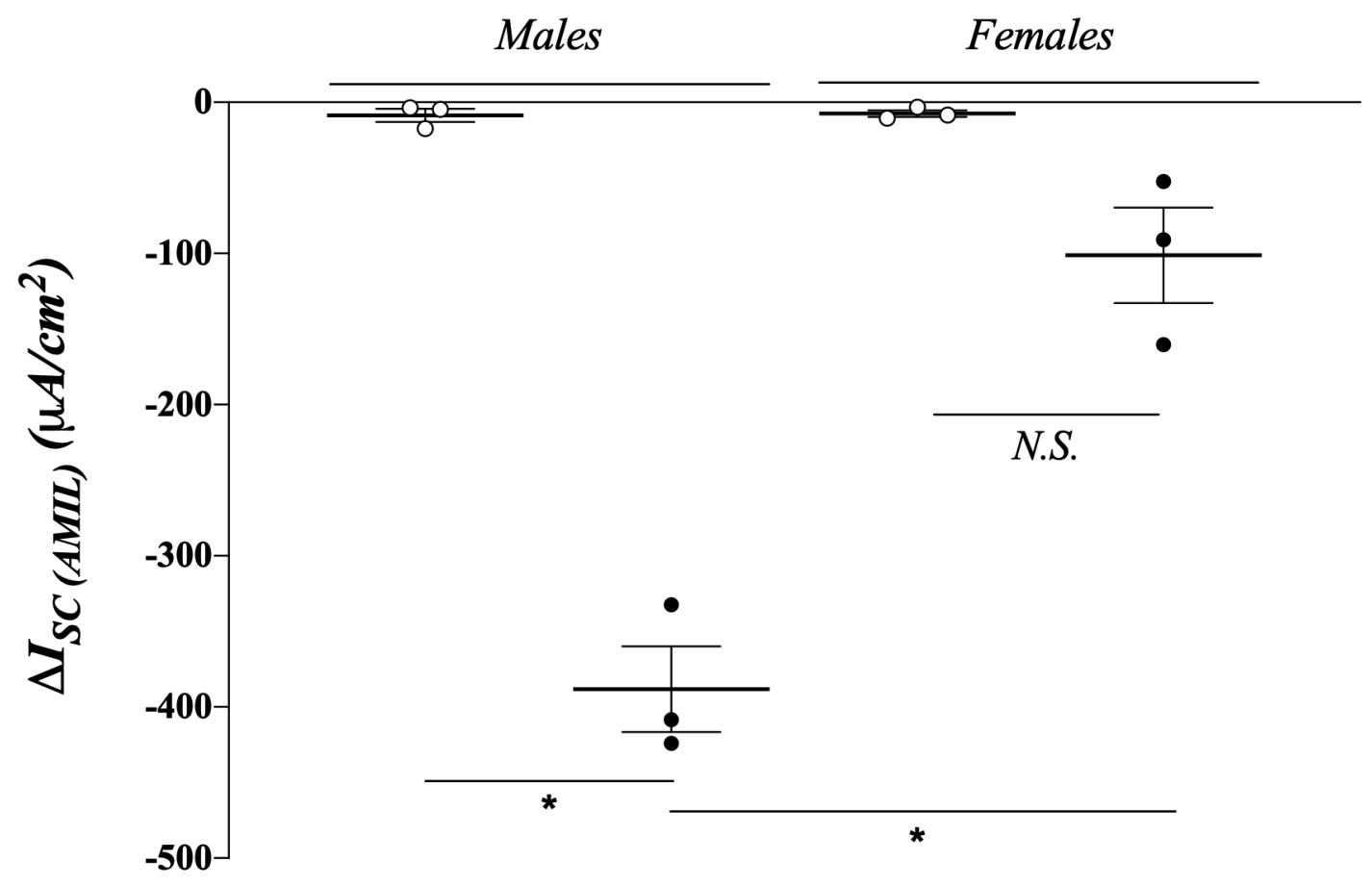




\section{Supplemental Figure 1. Dietary $\mathrm{Na}^{+}$depletion induces secondary hyperaldosteronism}

differentially in male versus female rats. (A) Aldosterone concentrations measured in serum collected from male and female rats fed normal diet (open circles) or $\mathrm{Na}^{+}$- deficient diet (closed circles) for six consecutive days. (B) Change in short-circuit current $\left(\Delta \mathrm{I}_{\mathrm{SC}}\right)$ induced by mucosal amiloride $(100 \mu \mathrm{M})$ in distal colonic mucosae isolated from male and female rats fed rats fed normal or $\mathrm{Na}^{+}$- deficient diet, measured in an Ussing-style recording chamber. ${ }^{*} p<0.05$ as determined by two-way ANOVA with Tukey's post-hoc test. Lines and error bars represent means \pm SEM. 


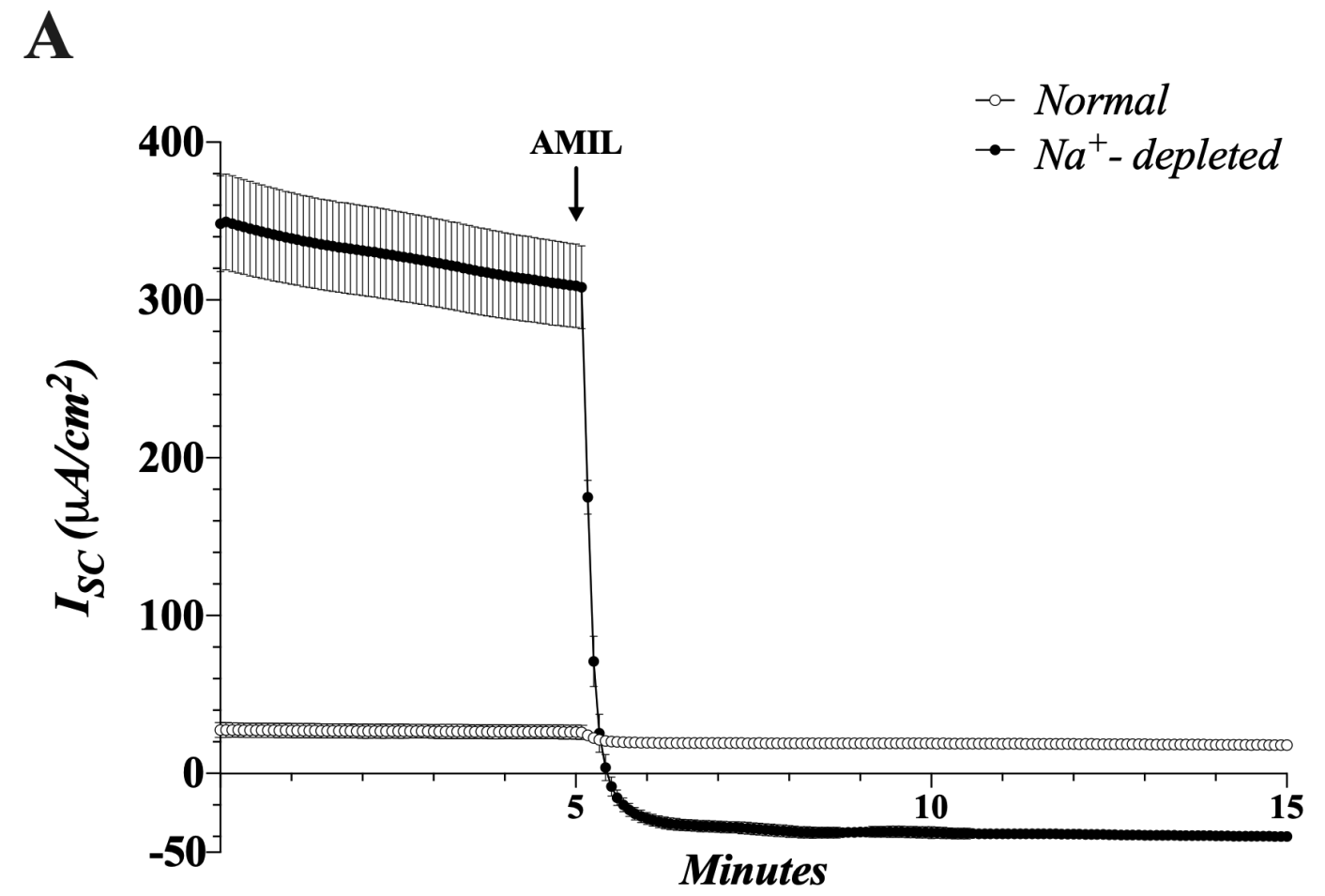

B

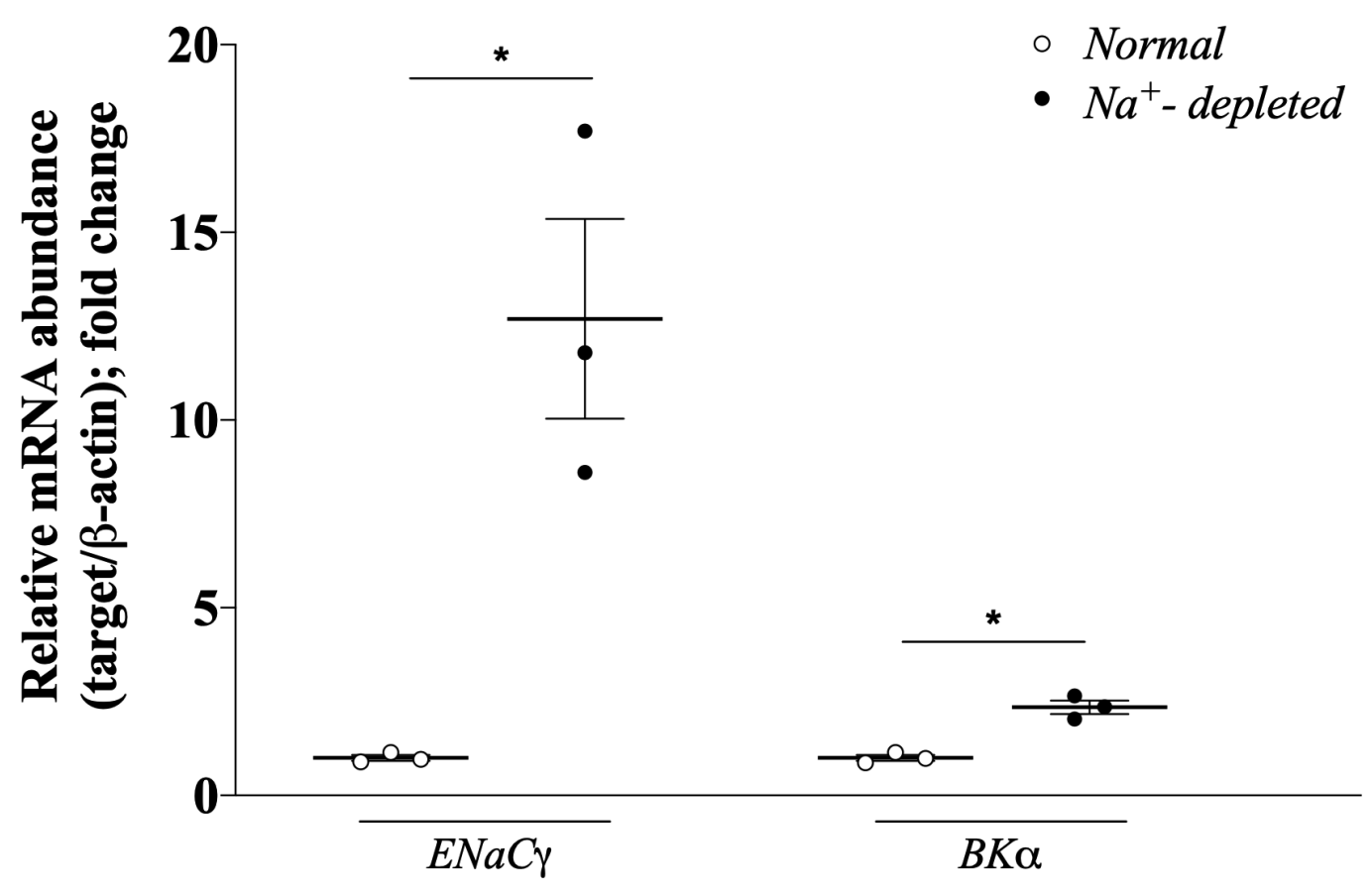




\section{Supplemental Figure 2. Dietary $\mathrm{Na}^{+}$depletion induces electrogenic $\mathrm{Na}^{+}$absorption and $\mathrm{K}^{+}$}

secretion. (A) Short circuit current $\left(\mathrm{I}_{\mathrm{SC}}\right)$ measured in mucosae from normal (open circles) and dietary $\mathrm{Na}^{+}$-depleted (closed circles) rat distal colon mounted under voltage clamp conditions. 100 $\mu \mathrm{M}$ amiloride (AMIL) was added to the mucosal bath where indicated. (B) ENaC $\gamma$ and $\mathrm{BK} \alpha$ mRNA abundance measured by qRT-PCR using total RNA isolated from normal and dietary $\mathrm{Na}^{+}$depleted rat distal colon. Lines and error bars represent means $\pm \mathrm{SEM},{ }^{*} p<0.01$, as determined by Student's unpaired t-test. 


\section{CHAPTER 5}

TITLE: Aldosterone up-regulates NKCC1 expression and enhances electrogenic $\mathrm{Cl}^{-}$secretion in rat proximal colon.

RUNNIG TITLE: Aldosterone enhances proximal colon $\mathrm{NKCC} 1$ and $\mathrm{Cl}^{-}$secretion.

AUTHORS: Andrew J. Nickerson ${ }^{1,2}$ and Vazhaikkurichi M. Rajendran ${ }^{2,3, *}$

1Departments of Physiology, Pharmacology and Neuroscience, West Virginia University School of Medicine, Morgantown, West Virginia

2Department of Biochemistry, West Virginia University School of Medicine, Morgantown, West Virginia

3Department of Medicine, West Virginia University School of Medicine, Morgantown, West Virginia

VMR ORCID: 0000-0002-8999-8721

AJN and VMR conceived of and designed the experiments. AJN performed all experiments and wrote the manuscript. AJN and VMR edited and prepared the manuscript in its current version.

* Address all correspondence

Vazhaikkurichi M. Rajendran, $\mathrm{PhD}$.

Departments of Biochemistry and Medicine

West Virginia University School of Medicine

1 Medical Center Drive

Morgantown, WV 26506

Phone: (304) 293-0510

Email: vrajendran@hsc.wvu.edu 


\section{ABSTRACT:}

Colonic electrolyte transport is regulated by aldosterone, which markedly enhances luminal $\mathrm{K}^{+}$secretion throughout the colon. Previous studies demonstrated that the basolateral $\mathrm{K}^{+}$ loader, $\mathrm{NKCC1}$, was transcriptionally regulated by aldosterone in the distal colon specifically to support $\mathrm{K}^{+}$secretion. Although $\mathrm{NKCC1}$ also is required to support luminal $\mathrm{Cl}^{-}$secretion throughout the gastrointestinal tract, its function in the distal colon appears restricted to the $\mathrm{K}^{+}$ secretory pathway. Major segmental variability exists between the proximal and distal regions of the colon, especially concerning the effect of aldosterone on various electrolyte transport systems. Experiments were therefore designed to determine whether aldosterone also up-regulates NKCC1 in the proximal colon to support $\mathrm{K}+$ secretion. Using dietary $\mathrm{Na}+$ depletion as a model of secondary hyperaldosteronism, we found that while NKCC1 protein expression was enhanced, electrogenic $\mathrm{K}+$ secretion was not inhibited by either bumetanide (NKCC1 inhibitor) or $\mathrm{Ba}^{2+}$ (nonselective $\mathrm{K}^{+}$channel blocker). In contrast, basal and stimulated $\mathrm{Cl}^{-}$secretion, as well as $\mathrm{CFTR}^{-}$ channel protein expression was significantly enhanced by aldosterone. These data suggest that the function of $\mathrm{NKCC} 1$ and its regulation by aldosterone differs between proximal and distal regions of the colon. Development of therapeutic strategies in treating pathologies related to aberrant colonic $\mathrm{K}^{+}$and/or $\mathrm{Cl}^{-}$transport - such as colonic pseudo-obstruction or ulcerative colitis - may benefit from the findings of this study.

NEW AND NOTEWORTHY: This study describes a novel mechanism by which aldosterone differentially regulates $\mathrm{K}^{+}$and $\mathrm{Cl}^{-}$transport in the proximal versus distal colon. In the proximal colon, $\mathrm{NKCC} 1$ expression is enhanced by aldosterone and correlates with increased $\mathrm{Cl}^{-}$secretion and CFTR expression. This differs from the distal colon, where NKCC1 is enhanced along with 
BK channels to support $\mathrm{K}^{+}$, but not $\mathrm{Cl}$ - secretion. These findings may be relevant to pathologies involving aberrant colonic $\mathrm{K}^{+}$and/or $\mathrm{Cl}^{-}$transport.

KEY WORDS: Mineralocorticoid, $\mathrm{Na}^{+}$depletion, large intestine, Ussing chamber 


\section{INTRODUCTION:}

Aldosterone is a critical regulator of colonic epithelial ion transport, serving to enhance $\mathrm{Na}^{+}$and water absorption and $\mathrm{K}^{+}$secretion (1-3). The effects of aldosterone on colonic transport processes are known to vary substantially by anatomical region within the colon. For example, aldosterone completely inhibits electroneutral $\mathrm{NaCl}$ absorption and stimulates electrogenic $\mathrm{Na}^{+}$ absorption through the epithelial $\mathrm{Na}^{+}$channel $(\mathrm{ENaC})$ in the distal colon (4-6), but it enhances electroneutral $\left(\mathrm{Na}^{+} / \mathrm{H}^{+}\right.$exchanger 3; NHE-3)-dependent $\mathrm{Na}^{+}$absorption in the proximal colon $(7,8)$. With respect to $\mathrm{K}^{+}$transport, aldosterone enhances $\mathrm{K}^{+}$secretion in both distal and proximal regions of the colon $(1,9,10)$, although there may be differences in the secretory pathways and channels/transporters involved (11). In addition, apical membrane $\mathrm{H}^{+} / \mathrm{K}^{+}$ATPases, which mediate active $\mathrm{K}^{+}$absorption, are found specifically in the distal colon $(12,13)$ and are also transcriptionally up-regulated by aldosterone (14).

In the distal colon, expression of the apical membrane large conductance $\mathrm{K}^{+}(\mathrm{BK})$ channel is increased by aldosterone (15-17), correlating to BK-mediated $\mathrm{K}^{+}$secretion as measured by isotopic flux assays (18) or electrophysiological recordings (16,19). Aldosterone-induced apical $\mathrm{K}^{+}$secretion is supported by basolateral $\mathrm{K}^{+}$uptake via $\mathrm{Na}^{+} / \mathrm{K}^{+}$ATPase and the $\mathrm{Na}^{+} / \mathrm{K}^{+} / 2 \mathrm{Cl}^{-}$cotransporter (NKCC1), NKCC1 (17). ${ }^{86} \mathrm{Rb}^{+}$flux experiments have established that net $\mathrm{K}^{+}$secretion is also enhanced by aldosterone in the proximal colon $(9,20)$. Still, the mechanism of aldosteroneinduced $\mathrm{K}^{+}$secretion in the proximal colon is not known, apart from recent functional and immunohistochemical evidence against a role for aldosterone in the regulation of BK channels or BK-mediated $\mathrm{K}^{+}$currents in rat proximal colon (11).

Recently, NKCC1, which facilitates basolateral $\mathrm{K}^{+}$uptake, was shown to be functionally up-regulated by aldosterone via the prevention of its proteasomal degradation in colonic epithelial 
cells in vitro $(21,22)$. Our recent studies, however, have demonstrated that aldosterone transcriptionally enhances $\mathrm{NKCC} 1$ expression both in vivo and ex vivo, specifically to support $\mathrm{K}^{+}$ secretion through the apical membrane BK channel in rat distal colon (23). However, it is unknown whether or not aldosterone controls $\mathrm{NKCC} 1$ expression and/or function within the proximal region of the tissue. The present study was therefore initiated to test the hypothesis that aldosterone upregulates $\mathrm{NKCC} 1$ to support enhanced $\mathrm{K}^{+}$secretion in rat proximal colon. Our results indicate that NKCC1 expression is upregulated by aldosterone, but this does not support electrogenic $\mathrm{K}^{+}$ secretion. Rather, basal and agonist-stimulated $\mathrm{Cl}^{-}$secretion is enhanced by aldosterone concurrently with NKCC1 upregulation, highlighting a novel aspect of the segmental variability in aldosterone function within the colon.

\section{METHODS:}

\section{Animals}

Male Sprague-Dawley rats were housed 2 per cage and maintained in 12-hour light/dark cycles in temperature-controlled housing. Rats were randomly assigned to groups receiving either standard rodent chow or $\mathrm{Na}^{+}$-deficient diet (MP Biomedicals \#02960364) for 6-7 days to induce secondary hyperaldosteronism, as has been described previously $(2,10,24,25)$. All rats were allowed free access to water throughout. On the day of experiments, rats were rapidly anesthetized with $5 \%$ isoflurane and maintained under a deep plane of anesthesia with $3 \%$ isoflurane delivered via nose cone. Colons were removed by cutting away mesenteric attachments and excising tissue from the caecum to the rectum and subsequently flushed with ice cold saline prior to use in downstream experiments. After tissue removal, rats were euthanized by cutting the diaphragm. All 
procedures were approved by West Virginia University's Institutional Animal are and Use Committee prior to the start of the project.

\section{Ussing chamber experiments}

After flushing, the colon was placed on a dissection stage and submerged in ice cold $\mathrm{HCO}_{3}{ }^{-}$ Ringer's solution containing the following, in $\mathrm{mM}: 140 \mathrm{Na}^{+}, 119.8 \mathrm{Cl}^{-}, 25 \mathrm{HCO}_{3}^{-}, 5.2 \mathrm{~K}^{+}, 1.2 \mathrm{Ca}^{2+}$, $1.2 \mathrm{Mg}^{2+}, 2.4 \mathrm{HPO}_{4}{ }^{2-}, 0.4 \mathrm{H}_{2} \mathrm{PO}_{4}^{-}$, and 10 glucose. The solution was continuously bubbled with $5 \% \mathrm{CO}_{2}$ balanced with oxygen to maintain $\mathrm{pH}$ at 7.41. The colon was opened with surgical scissors along the mesenteric border and placed mucosal side facing downward. A lateral incision was made with a razor blade just beneath the longitudinal and circular smooth muscle layers, which were peeled away to leave a submucosa/muscularis mucosae/mucosa preparation.

Segments of proximal colon, identified by striation patterns in the tissue, were then mounted on $1.1 \mathrm{~cm}^{2}$ plastic sliders for use in an Ussing-style recording chamber (Physiologic Instruments, San Diego CA). The recording chambers were equipped with one pair of voltagesensing $\mathrm{AgCl}$ pellet electrodes and one pair of current-injecting $\mathrm{AgCl}$ wire electrodes, each connected to the chamber bath via agar salt bride $(3.5 \%$ agar in $3 \mathrm{M} \mathrm{KCl})$. The chamber bath itself was temperature controlled contained $5 \mathrm{~mL}$ of bubbled $\mathrm{HCO}_{3}{ }^{-}$Ringer's solution in each chamber half. A multichannel voltage clamp (VCC MC8, Physiologic Instruments) was operated automatically by computer software to measure short-circuit current $\left(\mathrm{I}_{\mathrm{SC}}\right)$, a function of electrogenic ion transport. By convention, positive $\mathrm{I}_{\mathrm{SC}}$ values correspond to anion secretion and/or cation absorption. Intermittent bi-polar $200 \mathrm{~ms}, 5 \mathrm{mV}$ pulses were applied to monitor transepithelial conductance $\left(\mathrm{G}_{\mathrm{TE}}\right)$ at 30 second intervals.

For ${ }^{86} \mathrm{Rb}^{+}$flux experiments, $2 \mu \mathrm{Ci}$ of isotope was added to either the mucosa or serosal chamber bath in tissue pairs matched by conductance values within $20 \%$ of each other (26). 
Following a 20 -minute equilibration period, $500 \mu \mathrm{L}$ samples were taken from the chamber bath opposite to where the isotope was added (i.e., the "sink" side), as well as $10 \mu \mathrm{L}$ from the isotope bath ("hot" side) to be used in the calculation of basal $\mathrm{K}^{+}$transport. Samples were mixed with scintillation cocktail solution (Sigma Aldrich, \#03999-5L) and counted using a Tri-carb 4910TR liquid scintillation counter (Perkin Elmer, Waltham MA). Unidirectional ${ }^{86} \mathrm{Rb}^{+}$flux rates were calculated from the measured counts per minute (cpm) values using the following equation:

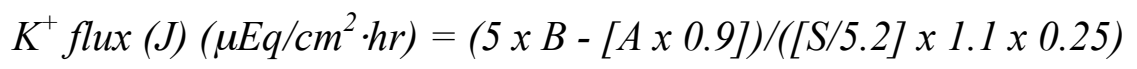

"A" and "B" represent $\mathrm{cpm} / \mathrm{mL}$ values measured from the beginning and end of the flux period, respectively. 5 is the total volume (in $\mathrm{mL}$ ) of the chamber bath and 0.9 is dilution factor accounting for removal of $500 \mu \mathrm{L}$ of bath volume upon sample collection. "S" represents the $\mathrm{cpm} / \mu \mathrm{Eq}$ (in $1 \mathrm{~mL}$ ) measured from the standard obtained from the "hot" bath of the chamber. 5.2 is the $\mathrm{K}^{+}$concentration in the bath $(\mathrm{mM}=\mathrm{mMol} / \mathrm{L}=\mu \mathrm{Eq} / \mathrm{mL}), 1.1$ corresponds to the slider aperture (in $\mathrm{cm}^{2}$ ) and 0.25 is the time (in hours) for the flux period (26). Net $\mathrm{K}^{+}$fluxes were calculated by subtracting the serosal to mucosal $(S-M)$ from the mucosal to serosal $(M-S)$ fluxes from paired tissues.

\section{Mucosal Scraping}

Proximal colon sacs were made by filling with ice cold buffer containing (in $\mathrm{mM}$ ): 15.4 $\mathrm{NaCl}, 8$ HEPES/Tris (pH 7.5), 5 ethylenediaminetetraacetic acid (EDTA), 0.5 dithiothreitol (DTT), and 0.5 phenylmethylsulphonyl fluoride (PMSF) and incubated in the same solution on ice for 45 minutes. The drained sacs were opened along the mesenteric border. The proximal mucosal 
layer was scraped off using glass slides and frozen immediately in liquid nitrogen until use. Scrapped mucosae were used for RNA isolation and protein extraction.

\section{RNA isolation and $q R T-P C R$ analysis}

Total RNA was isolated from frozen colonic mucosa $(50 \mathrm{mg})$ using the Trizol method and quantitated with NanoDrop spectrometer (Thermo Sci. ND2000C). Quantitative reverse transcriptase polymerase chain reaction (qRT-PCR) was performed using a One-Step reaction kit (NEB \#E3005) per the manufacturer's protocol. Briefly, custom gene-specific primers were designed using the published DNA sequence (NCBI database) and synthesized by Thermo Scientific. Primer sequences are listed in Table 1. qRT-PCR reactions performed on a 96-well plate were read using a CFX96 real time PCR machine (BioRad). mRNA abundance was determined using the delta-delta $C_{t}$ method, with $\beta$-actin as a reference housekeeping gene and represented in the figures as fold change in mRNA transcript expression in the experimental $\left(\mathrm{Na}^{+}\right.$-depleted, "aldo") relative to the control (normal) animals.

\section{Western blot analysis}

Proteins were extracted from frozen colonic mucosae $(50 \mathrm{mg})$ by homogenizing in ice-cold lysis buffer $(150 \mathrm{mM} \mathrm{NaCl}, 50 \mathrm{mM}$ Tris base, $0.5 \%$ sodium deoxycholate, $0.1 \% \mathrm{SDS}, 1.0 \%$ Triton-X100) plus protease inhibitor cocktail (Thermo \#A32965), followed by a brief sonication (Fisher Sonic Dismembranator Model 100). Samples were centrifuged for 20 minutes at 12,000 x $\mathrm{g}$ and the supernatant was mixed with 4x Laemmli buffer (Life Technologies \#NP0007) and heated to $90^{\circ} \mathrm{C}$ for 5 minutes. Samples were then chilled on ice before adding $\beta$-mercaptoethanol $(5 \%$ $\mathrm{w} / \mathrm{v})$, and the aliquoted samples were flash frozen and stored at $-80^{\circ} \mathrm{C}$. Proteins $(\sim 20 \mu \mathrm{g})$ were 
resolved on $8 \%$ Tris-glycine polyacrylamide gels and transferred on to PVDF membranes. Membranes were incubated in blocking buffer (3\% BSA in tris-buffered saline plus $0.1 \%$ Tween20 (TBS-T) for 1 hour at room temperature, followed by overnight incubation at $4^{\circ} \mathrm{C}$ in primary antibody solution. Table 2 provides the source and dilutions used for all primary antibodies. The membranes were then washed in TBST ( 5 minutes $\mathrm{x} 5$ ) and incubated in TBST $+3 \%$ BSA containing goat anti-rabbit HRP-conjugated secondary antibody (Thermo \#31462; 1:20,000 dilution) for 1 hour at room temperature before being washed again in TBST ( 5 min $x$ 5). Immune complexes were detected using enhanced chemiluminescence (West Dura, Thermo \#34075). Images were captured using a G:BOX (Syngene) and band intensities were quantified by densitometry using Image $\mathrm{J}$ software and normalized to $\beta$-actin as a loading control.

\section{Immunofluorescence microscopy}

Segments of whole proximal colon were fixed overnight at $4^{\circ} \mathrm{C}$ in $4 \%$ paraformaldehyde.

The next day, tissues were washed in PBS, flash frozen in isopentane cooled with liquid nitrogen and embedded in tissue freezing medium. $10 \mu \mathrm{m}$ sections were cut with a cryostat and mounted on charged glass slides. 40 minutes of heated antigen retrieval was performed by placing the slides in $10 \mathrm{mM}$ citrate $+0.05 \%$ Tween-20 buffer and microwaving on very low power for 45 minutes before allowing gradual cooling to room temperature. Sections were then permeabilized in PBS + $5 \%$ goat serum $+0.1 \%$ Triton-X for 15 minutes. Afterward, primary antibody solution containing PBS $+0.01 \%$ Triton-X $(\mathrm{PBST})+5 \%$ goat serum with a 1:500 dilution of NKCC1 primary antibody (Cell Signaling \#14581) $(23,27)$ was placed on the sections overnight at $4^{\circ} \mathrm{C}$. The next day, tissues were washed 5 times for 5 minutes each in PBST and subsequently incubated in PBST $+5 \%$ goat serum containing goat anti-rabbit AlexaFluor 488 secondary antibody (Thermo \#11008; 1:2,00 dilution) for 1 hour at room temperature. After washing an additional 5 times for 5 minutes each, 
slides were mounted with SlowFade Diamond antifade mountant with DAPI (Thermo \#36971) and sealed with clear fingernail polish. IF images were captured using a Zeiss 710 confocal microscope at 20x magnification (Carl Zeiss, Oberkochen, Germany).

\section{RESULTS:}

Aldosterone (dietary $\mathrm{Na}^{+}$depletion) enhances net $\mathrm{K}^{+}$secretion in the proximal colon.

Preliminary experiments were conducted to confirm that net $\mathrm{K}^{+}$secretion in the rat proximal colon was enhanced by aldosterone. Unidirectional ${ }^{86} \mathrm{Rb}^{+}$fluxes were measured as described above using tissues from normal or dietary $\mathrm{Na}^{+}$-depleted rats. Dietary $\mathrm{Na}^{+}$depletion is a well-established model of secondary hyperaldosteronism that has been extensively characterized (24). Throughout this study, dietary $\mathrm{Na}^{+}$-depleted rats are referred to as "aldo" (i.e., hyperaldosteronaemic). Table 3 depicts the results from ${ }^{86} \mathrm{Rb}^{+}$flux experiments using normal and aldo proximal colonic tissues. Net $\mathrm{K}^{+}$secretion was found to be enhanced in aldo rats via an increase in the serosal-to-mucosal flux ( $p<0.01$ for both net and S-M fluxes). Short circuit current ( $\left.\mathrm{I}_{\mathrm{SC}}\right)$ and transepithelial conductance $\left(\mathrm{G}_{\mathrm{TE}}\right)$ were also higher in aldo tissues as well. These data are consistent with previous demonstrations $(10,20)$ and provide the basis for further investigation into the role of $\mathrm{NKCC} 1$ in these processes.

NKCC1 protein expression is enhanced in "Aldo" proximal colon.

Western blot analysis of normal and aldo rat proximal colon revealed a $\sim 2$-fold increase in NKCC1 protein abundance (Fig. 1 A-B) $(p<0.05)$, but no significant change in mRNA expression was detected by qRT-PCR (Fig. 1C) $(p=0.32)$. Confocal immunofluorescence microscopy images qualitatively reflect the results from western blot analysis (Fig. 2), as NKCC1-specific labelling 
was more prominent in aldo proximal colon sections, particularly in the crypt region. The observed $\sim 2$-fold increase in NKCC1 protein abundance (Fig. 1) mirrors the response previously described in the distal colon using the same model system (23). However, the discrepancy here between changes in NKCC1 mRNA transcript and protein abundance in the proximal colon suggests that the upregulation of $\mathrm{NKCC} 1$ in this region may not be the result of a transcriptional mechanism, as was previously demonstrated in the distal colon. While the nature of this phenomenon was not further explored here, a similar pattern has been observed in cultured epithelial cells in response to aldosterone and is likely attributable to changes in the proteasomal pathway $(21,22)$, providing a possible explanation for the findings.

Electrogenic $\mathrm{Ba}^{2+}$ - or bumetanide-sensitive $\mathrm{K}^{+}$secretion is not present in normal or aldo rat proximal colon.

After establishing that aldo rats exhibit increased net $\mathrm{K}^{+}$secretion as well as increased $\mathrm{NKCC} 1$ protein expression, experiments were next initiated to delineate the role of $\mathrm{NKCC} 1$ in supporting electrogenic $\mathrm{K}^{+}$secretion. Here, electrogenic $\mathrm{K}^{+}$secretion was measured as $\mathrm{I}_{\mathrm{SC}}$ (luminal cation secretion being a negative by convention) because ${ }^{86} \mathrm{Rb}^{+}$was taken off the market during the course of these studies. Agents that inhibit electrogenic $\mathrm{K}^{+}$secretion in the Ussing chamber produce positive deflections in $\mathrm{I}_{\mathrm{SC}}$, corresponding to the inhibition of "negative" current. This method of quantifying electrogenic $\mathrm{K}^{+}$secretion has been widely utilized $(16,28)$.

Surprisingly, neither mucosal $\mathrm{Ba}^{2+}$ (non-selective $\mathrm{K}^{+}$channel blocker) nor serosal bumetanide (NKCC1 inhibitor) inhibited basal $\mathrm{K}^{+}$secretion (Fig. 3). Mucosal $\mathrm{Ba}^{2+}$ actually induced a slightly but significantly more negative deflection in $\mathrm{I}_{\mathrm{SC}}$ (Normal vs. Aldo: $-1.5 \pm 2.6$ vs. $-12.7 \pm 3.7 \mu \mathrm{A} / \mathrm{cm}^{2} ; p<0.05$ ) (Fig. 3A,D), possibly corresponding to an inhibition of mucosal 
$\mathrm{Cl}^{-}$secretion, although $\mathrm{G}_{T E}$ was not also noticeably affected (Fig. 3B). Previous studies in our own lab using the distal region of the colon have shown a maximal effect of mucosal $\mathrm{Ba}^{2+}$ addition within 5 minutes of application $(16,23)$, indicating the lack of a response here was not likely due to the requirement of a longer time period.

Subsequent application of bumetanide to the serosal chamber bath produced a relatively small and transient decrease in $\mathrm{I}_{\mathrm{SC}}$ in aldo tissues that was not as pronounced in normal tissues (Normal vs. Aldo $\Delta \mathrm{I}_{\mathrm{SC}}$ (initial): $-2.1 \pm 0.8$ vs. $-10.3 \pm 2.2 \mu \mathrm{A} / \mathrm{cm}^{2} ; p<0.01$ ) (Fig. 3A,E), possibly corresponding to an initial reduction in $\mathrm{Cl}^{-}$secretion. However, this effect was again very minimal and unaccompanied by a parallel decrease in $\mathrm{G}_{\mathrm{TE}}$ (Fig. 3B). In addition, the response to bumetanide was not sustained, as the steady-state change in I $\mathrm{SC}_{\mathrm{SC}}$ was not significantly different between normal and aldo tissues (Normal vs. Aldo $\Delta \mathrm{I}_{\mathrm{SC}}$ (plateau): $8.7 \pm 5.1$ vs. $1.3 \pm 3.3 \mu \mathrm{A} / \mathrm{cm}^{2} ; p=0.26$ ) (Fig. 3E). The relatively small effect of serosal bumetanide in both normal and aldo rat tissues may be the result of an inability of the drug to completely penetrate the submucosa, which was still intact in these preparations. Distal colonic tissues can be prepared to contain only mucosa and muscularis mucosae, allowing greater access to the basolateral membranes, and this is reflected in a more robust response to bumetanide. However, anatomical differences make this type of tissue preparation extremely difficult, if not impossible, in the proximal colon.

\section{Mucosal BK and IK channel expression is not enhanced by aldosterone in rat proximal colon.}

We next sought to determine whether mucosal (apical) membrane $\mathrm{K}^{+}$channels were detectable in proximal colon tissue lysates and whether or not their expression was altered by aldosterone. Perry et. al. recently reported the presence of large conductance $\mathrm{K}^{+}(\mathrm{BK})$ channels in patch clamp recording from proximal colonocytes (11). In their study, hyperaldosteronism induced 
by dietary $\mathrm{K}^{+}$loading (an alternative model to dietary $\mathrm{Na}^{+}$depletion) did not increase the number of functional $\mathrm{K}^{+}$channels in the proximal colon detectable by patch clamp, western blot or immunohistochemistry. This was in sharp contrast to the effect observed in the distal colon.

Expression of intermediate conductance $\mathrm{K}^{+}$(IK) channels, which are also present in the proximal colon in rat, was not quantified in that study. Further, because the models of dietary $\mathrm{Na}^{+}$ depletion and $\mathrm{K}^{+}$loading are not equivalent, quantification of both BK and IK channel expression was performed here. Neither BK nor IK abundance was found to be altered in aldo animals (Fig. 4), as detected by Western blot (Fig. 4A-C) or qRT-PCR (Fig. 4D,E), corroborating the findings of Perry et. al. Although $\mathrm{Ba}^{2+}$-sensitive $\mathrm{K}^{+}$secretion was not observed in either normal or aldo proximal colon, both BK and IK channels were detected at the mRNA and protein level. Therefore, BK or IK channels either do not facilitate mucosal $\mathrm{K}^{+}$secretion in the proximal colon, or there exists some structural/functional difference between proximal and distal tissues, rendering these channels insensitive to mucosal $\mathrm{Ba}^{2+}$. Even if some other yet-unidentified $\mathrm{K}^{+}$channel was involved (e.g., of a voltage-gated family), a concentration $3 \mathrm{mM} \mathrm{Ba}^{2+}$ would almost certainly be sufficient to inhibit its activity.

Basal, $\mathrm{Ca}^{2+}$ - and cAMP-stimulated $\mathrm{Cl}^{-}$secretion is enhanced by aldosterone in the proximal colon.

NKCC1 is known to support aldosterone-induced $\mathrm{K}^{+}$secretion, specifically, in the distal colon $(17,23,29)$ and other tissues $(22,30)$. In the distal colon of aldo rats, basal $\mathrm{I}_{\mathrm{SC}}$ is negative in the presence of mucosal amiloride (ENaC inhibitor) $(31,32)$, indicating the presence of basal $\mathrm{K}^{+}$ secretion and minimal $\mathrm{Cl}^{-}$secretion, if any. These electrical properties are supported by isotopic flux data (2). However, in the rat proximal colon, basal $\mathrm{I}_{\mathrm{SC}}$ is increased by aldosterone (Table1, Fig. 3) (7). Therefore, $\mathrm{Cl}^{-}$secretion ostensibly predominates over the simultaneous $\mathrm{K}^{+}$secretion 
that is also apparently present, as indicated by ${ }^{86} \mathrm{Rb}^{+}$flux experiments. Indeed, the most welldefined role of $\mathrm{NKCC} 1$ throughout the gastrointestinal tract is that of supporting basal and stimulated $\mathrm{Cl}^{-}$secretion to the lumen $(33,34-36)$. Thus, we next assessed whether the aldosteroneinduced increase in $\mathrm{NKCC} 1$ expression correlated to an increased capacity for $\mathrm{Cl}^{-}$secretion.

Aldo proximal colonic tissues once again exhibited higher basal $\mathrm{I}_{\mathrm{SC}}$ (i.e., $\mathrm{Cl}^{-}$secretion) compared to tissues from normal rats (Normal vs. Aldo $\mathrm{I}_{\mathrm{SC}}: 45.7 \pm 7.3 \mathrm{vs} .75 .1 \pm 8.0 \mu \mathrm{A} / \mathrm{cm}^{2}$; $p<0.05)$ (Fig. 5 A-C). The secretory responses to carbachol $(\mathrm{CCH}$; cholinergic agonist) and forskolin (FSK; adenylyl cyclase activator) were also enhanced by aldosterone (Normal vs. Aldo $\Delta \mathrm{I}_{\mathrm{SC}}(\mathrm{CCH}): 110.2 \pm 29.8$ vs. $\left.229.2 \pm 14.5 \mu \mathrm{A} / \mathrm{cm}^{2} ; p<0.01\right)$ (Fig. 5D); (Normal vs. Aldo $\Delta \mathrm{I}_{\mathrm{SC}}$ (FSK): $114.6 \pm 16.1$ vs. $\left.157.5 \pm 9.2 \mu \mathrm{A} / \mathrm{cm}^{2} ; p<0.05\right)$ (Fig. $\left.5 \mathrm{E}\right)$. These data suggest that aldosterone increases both basal $\mathrm{Cl}^{-}$secretion and the capacity to respond to either $\mathrm{Ca}^{2+}$ - or cAMP-inducing secretory agonists (e.g., neurotransmitters or paracrine signals).

CFTR channel protein expression is also enhanced by aldosterone in rat proximal colon.

Most, if not all, luminal $\mathrm{Cl}^{-}$secretion in the gastrointestinal tract is mediated by apical membrane-localized Cystic Fibrosis transmembrane conductance regulator (CFTR) $\mathrm{Cl}^{-}$channels (37-40). TMEM16A, a $\mathrm{Ca}^{2+}$-activated $\mathrm{Cl}^{-}$channel, is also likely involved (40-42), but does not appear to mediate $\mathrm{Cl}^{-}$efflux across the apical membrane. Because basolateral NKCC1 protein expression and basal/stimulated $\mathrm{Cl}^{-}$secretion were all enhanced by aldosterone in the proximal colon, we sought to determine if the expression of either of these two $\mathrm{Cl}^{-}$channel proteins was increased in conjunction.

Western blot analysis revealed that CFTR protein, and in particular the mature, glycosylated form ("c" band) (43) was increased $\sim 1.5$ fold in aldo rat proximal colonic mucosae 
(Fig. 6A,D). Similar to NKCC1, the mRNA transcript abundance was not significantly increased either (Fig. 6E). Interestingly, TMEM16A transcript (Fig. 6E) but not protein (Fig. 6B,D) was detected in any of these samples. The TMEM16A antibody used here has been validated previously by our lab and others $\left({ }^{44}\right)$, and as a positive control, distal colonic mucosae tissue lysates were probed alongside proximal colon samples. TMEM16A was detected only in the distal colon (Fig. 6C), suggesting possible regional variability in the functional role of TMEM16A.

\section{DISCUSSION:}

Our findings indicate that the expression of the basolateral NKCC1 co-transporter was enhanced by aldosterone (dietary $\mathrm{Na}^{+}$depletion) in rat proximal colon. Further, this was correlated with an augmented capacity for electrogenic $\mathrm{Cl}^{-}$secretion, rather than $\mathrm{K}^{+}$secretion. This is in stark contrast to the effects of aldosterone on $\mathrm{K}^{+}$and $\mathrm{Cl}^{-}$transport processes in the distal colon - where $\mathrm{K}^{+}$, but not $\mathrm{Cl}^{-}$secretion is enhanced - despite similar effects concerning the expression of NKCC1. A parallel increase in the apical membrane $\mathrm{CFTR}^{-}$channel was observed, whereas BK and IK channel expression was found to be unaltered by aldosterone, which is also contrary to the effects seen in the distal colon. Segmental variability within the two regions of the colon has been well-documented in terms of the effects of corticosteroid hormones, including their influence of $\mathrm{Na}^{+}, \mathrm{K}^{+}$, and $\mathrm{Cl}^{-}$transport. Here, we have identified an additional novel example of heterogeneity in the regulation of colonic ion transport systems by aldosterone.

The results of this study are clinically relevant because colonic $\mathrm{K}^{+}$and $\mathrm{Cl}^{-}$transport is a critical aspect of many pathologies. For example, in patients with end-stage renal disease (ESRD), the colon provides an alternative outlet for $\mathrm{K}^{+}$which can prevent life-threatening hyperkalemia $(45,46)$. On the other hand, colonic pseudo-obstruction $(47)$ and ulcerative colitis (UC) $(12,48,49)$ 
are characterized by excessive fecal $\mathrm{K}^{+}$losses which contribute to the manifestation of diarrhea. Modulation of colonic ion transport via drugs that act upon the mineralocorticoid (i.e., aldosterone) receptor has even been used clinically in at least one case of pseudo-obstruction (Ogilvie's syndrome) (50). Other diarrheal pathologies such as enteroviral or enterobacterial infection primarily affect $\mathrm{Cl}^{-}$secretion independently of $\mathrm{K}^{+}$secretion $(51,52)$. However, given that such variability exists between colonic regions in terms of aldosterone-regulated $\mathrm{K}^{+}$and $\mathrm{Cl}^{-}$transport, results from this and related studies may help to determine the most appropriate course of action in managing these types of pathologies in patients with unique circumstances (e.g., partial colectomy).

The overall function of aldosterone signaling in the colon is to promote salt $(\mathrm{NaCl})$ and fluid retention, while also driving the removal of excess $\mathrm{K}^{+}$from the body (53). Thus, an increase in $\mathrm{NKCC1}$ expression which may be tied only to $\mathrm{Cl}^{-}$secretion appears counterproductive because up-regulation of this pathway would, in theory, hinder fluid absorption. However, $\mathrm{Cl}^{-}$absorption in the proximal colon is known to be electroneutral (mediated primarily by DRA) and occurs mostly in surface absorptive cells (7). On the other hand, NKCC1 and CFTR are known to be expressed primarily within the crypt region $(36) .{ }^{36} \mathrm{Cl}^{-}$flux studies in the past have shown an increase in net $\mathrm{Cl}^{-}$absorption within the proximal colon of aldo rats compared to controls. However, the magnitude of $\mathrm{Cl}^{-}$flux did not match that of $\mathrm{Na}^{+}(7,9)$, even though the transport of these two ions is functionally coupled through $\mathrm{Na}^{+} / \mathrm{H}^{+}$and $\mathrm{Cl}^{-} / \mathrm{HCO}_{3}{ }^{-}$exchangers. The increase in basal $\mathrm{I}_{\mathrm{SC}}$ (i.e., $\mathrm{Cl}^{-}$secretion) measured in those studies as well as ours, along with the augmented response to secretagogues (i.e., those potentially derived from basal neurogenic input) may account for the discrepancy in net $\mathrm{Na}^{+}$versus $\mathrm{Cl}^{-}$fluxes. That is to say, a portion of the $\mathrm{Cl}^{-}$absorbed through surface epithelial cells may ultimately be "recycled" back to the lumen via secretion occurring 
within the crypt. Despite this possible explanation, the physiological significance of having an enhanced capacity for $\mathrm{Cl}^{-}$secretion under hyperaldosteronaemic conditions remains unclear.

Another puzzling observation is that although the net secretion of $\mathrm{K}^{+}$is enhanced by aldosterone in the proximal colon, as indicated by ${ }^{86} \mathrm{Rb}^{+}$flux experiments (Table 3) (20), the nature of this luminally-directed $\mathrm{K}^{+}$transit pathway has yet to be identified. In particular, our data indicate that at least electrogenic $\mathrm{K}^{+}$secretion in both normal and aldo proximal colon is insensitive to $\mathrm{Ba}^{2+}$ - a non-selective $\mathrm{K}^{+}$channel blocker - because no effect on $\mathrm{I}_{\mathrm{SC}}$ was observed. Other experiments from our lab indicate that basal and cAMP-stimulated $\mathrm{K}^{+}$secretion in normal proximal colon is not inhibited by other $\mathrm{K}^{+}$channel inhibitors either (e.g., iberiotoxin, Tram-34, paxilline) as measured by $\mathrm{I}_{\mathrm{SC}}$ and ${ }^{86} \mathrm{Rb}$ fluxes (unpublished observations). The apparent insensitivity to $\mathrm{K}^{+}$ channel inhibitors/blockers, especially $\mathrm{Ba}^{2+}$, raises the possibility that luminal $\mathrm{K}^{+}$secretion in the proximal colon is not facilitated by a channel, but rather through some other secretory mechanism. Perry et. al. examined BK channel activity in normal and aldo ( $\mathrm{K}^{+}$-loaded) rat proximal colonocytes by whole-cell and single channel patch clamp recording (11). Although this approach allows for fine resolution of the biophysical properties of $\mathrm{K}^{+}$channels for the purpose of identifying specific channels, vectorial transport functions cannot be measured using this technique. Nonetheless, our data are congruent with the findings from that study, as BK (and IK) channels were detectable, but aldosterone had no apparent effect on their expression. It is possible that the function of these $\mathrm{K}^{+}$channels in the proximal colon is unrelated to luminal $\mathrm{K}^{+}$secretion. For example, they may be involved only in regulating colonocyte membrane potential so as to influence other ion transport pathways through modulation of electrochemical driving forces.

A recent study using transgenic mice revealed an important role for BK and its accessory subunit, LRRC26, specifically in goblet cells of the colon (54). Ablation of functional BK channels 
in goblet cells led to goblet cell deterioration and resulted in increased susceptibility to DSSinduced colitis, which is known to depend on goblet cell function and mucous barrier integrity. Another study concluded that a population of goblet cells was responsible for $\mathrm{K}+$ secretion in the colon (55). Importantly, goblet cells also express $\mathrm{NKCC} 1(36,56)$. Luminal $\mathrm{Cl}^{-}$secretion from crypt secretory cells is also believed to be tied to mucous and $\mathrm{HCO}_{3}{ }^{-}$secretion from goblet cells (57). The new light shed on the importance of goblet cell $\mathrm{K}^{+}$channels by Gonzalez-Perez et. al. may be a clue related to the apparently $\mathrm{Ba}^{2+}$-insensitive $\mathrm{K}^{+}$secretory mechanism described here. It may also point to a potential setting in which enhanced $\mathrm{NKCC} 1$ expression by aldosterone may be of practical importance: if goblet cells and mucous production in the proximal colon are stimulated by aldosterone, then NKCC1 expression may likely increase there, permitting enhanced $\mathrm{Cl}^{-}\left(\right.$or $\mathrm{HCO}_{3}{ }^{-}$) secretion through those goblet cells or adjacent crypt secretory cells. At the same time, luminal $\mathrm{K}^{+}$"secretion" may be occurring through degranulation and dumping of large intracellular, $\mathrm{K}^{+}$-rich fluid volumes to the luminal space instead of via facilitated diffusion through apical membrane $\mathrm{K}^{+}$channels. This may explain why $\mathrm{K}^{+}$channel inhibitors appear ineffective at blocking secretion. These predictions are purely speculative, however, as there have been no reports to our knowledge concerning the regulation of goblet cells by aldosterone. Many experiments will need to be performed to appropriately explore this possibility.

In conclusion, here we have presented novel evidence of the up-regulation of NKCC1 and $\mathrm{Cl}^{-}$secretory capacity by aldosterone in the rat proximal colon. This effect appears to be unrelated to electrogenic $\mathrm{K}^{+}$secretion, which differs from the effects of aldosterone on $\mathrm{K}^{+}$and $\mathrm{Cl}^{-}$transport in the distal colon. Segmental differences must be accounted for when dealing with aldosteroneinduced changes to colonic $\mathrm{K}^{+}$and $\mathrm{Cl}^{-}$transport processes. 


\section{REFERENCES:}

1. Foster ES, Jones WJ, Hayslett JP, Binder HJ. Role of Aldosterone and Dietary Potassium in Potassium Adaptation in the Distal Colon of the Rat. Gastroenterology. 1985;88(1):4146. doi:10.1016/S0016-5085(85)80130-X

2. Halevy J, Budinger ME, Hayslett JP, Binder HJ. Role of Aldosterone in the Regulation of Sodium and Chloride Transport in the Distal Colon of Sodium-Depleted Rats.

Gastroenterology. 1986;91(5):1227-1233. doi:https://doi.org/10.1016/S00165085(86)80021-X

3. Rechkemmer G, Halm DANR. Aldosterone stimulates K secretion across mammalian colon independent of Na absorption. Proc Natl Acad Sci. 1989;86(January):397-401.

4. Amasheh S, Epple HJ, Mankertz J, et al. Differential Regulation of ENaC by Aldosterone in Rat Early and Late Distal Colon. Ann N Y Acad Sci. 2006;915(1):92-94. doi:https://doiorg.wvu.idm.oclc.org/10.1111/j.1749-6632.2000.tb05227.x

5. Greig ER, Baker EH, Mathialahan T, Boot-Handford RP, Sandle GI. Segmental variability of $\mathrm{ENaC}$ subunit expression in rat colon during dietary sodium depletion. Pflugers Arch Eur J Physiol. 2002;444(4):476-483. doi:10.1007/s00424-002-0828-7

6. Binder HJ, Foster ES, Budinger ME, Hayslett JP. Mechanism of electroneutral sodium chloride absorption in distal colon of the rat. Gastroenterology. 1987;93(3):449-455. doi:10.1016/0016-5085(87)90905-X

7. Foster ES, Budinger ME, Hayslett JP, Binder HJ. Ion transport in proximal colon of the rat. Sodium depletion stimulates neutral sodium chloride absorption. $J$ Clin Invest. 1986;77(1):228-235. doi:10.1172/JCI112281

8. Ikuma M, Kashgarian M, Binder HJ, Rajendran VM. Differential regulation of NHE isoforms by sodium depletion in proximal and distal segments of rat colon. Am J Physiol Gastrointest Liver Physiol. 1999;276(2 39-2):539-549. doi:10.1152/ajpgi.1999.276.2.g539

9. Tumamian SG, Binder HJ. Regulation of active sodium and potassium transport in the distal colon of the rat. J Clin Invest. 1989;84(6):1924-1929.

10. Foster S, Hayslett JP, Binder J, Emily S, Hayslew JP, Henry J. Mechanism of active potassium absorption and secretion in the rat colon. 1984. 
11. Perry MD, Rajendran VM, MacLennan KA, Sandle GI. Segmental differences in upregulated apical potassium channels in Mammalian colon during potassium adaptation. Am J Physiol - Gastrointest Liver Physiol. 2016;311(5):G785-G793. doi:10.1152/ajpgi.00181.2015

12. Rajendran VM, Sandle GI. Colonic Potassium Absorption and Secretion in Health and Disease. Compr Physiol. 2018;8(4):1513-1536. doi:10.1002/cphy.c170030

13. Rajendran VM, Singh SK, Geibel J, Binder HJ. Differential localization of colonic H+$\mathrm{K}+-\mathrm{ATPase}$ isoforms in surface and crypt cells. Am J Physiol - Gastrointest Liver Physiol. 1998;274(2 37-2):424-429. doi:10.1152/ajpgi.1998.274.2.g424

14. Spicer Z, Clarke LL, Gawenis LR, Shull GE. Colonic H(+)-K(+)-ATPase in $\mathrm{K}(+)$ conservation and electrogenic $\mathrm{Na}(+)$ absorption during $\mathrm{Na}(+)$ restriction. Am J Physiol Gastrointest Liver Physiol. 2001;281(6):G1369-77. doi:10.1152/ajpgi.2001.281.6.G1369

15. Sausbier M, Matos JE, Sausbier U, et al. Distal colonic K+ secretion occurs via BK channels. J Am Soc Nephrol. 2006;17(5):1275-1282. doi:10.1681/ASN.2005101111

16. Sørensen M V, Matos JE, Sausbier M, et al. Aldosterone increases KCa1.1 (BK) channelmediated colonic K+ secretion. J Physiol. 2008;586(Pt 17):4251-4264. doi:10.1113/jphysiol.2008.156968

17. Sweiry JH, Binder HJ. Characterization of aldosterone-induced potassium secretion in rat distal colon. J Clin Invest. 1989;83(3):844-851. doi:10.1172/JCI113967

18. Singh SK, O’Hara B, Talukder JR, Rajendran VM. Aldosterone induces active K+ secretion by enhancing mucosal expression of Kcnn4c and Kcnmal channels in rat distal colon. Am J Physiol - Cell Physiol. 2012;302(9):1353-1360.

doi:10.1152/ajpcell.00216.2011

19. Lomax B, Mcnicholas M, Lombes M, Sandle I, Mcnicholas M. Aldosterone-induced apical $\mathrm{Na}+$ and $\mathrm{K}+$ conductances are located primarily in surface cells in rat distal colon. 1994;29:G71-G82.

20. Turnamian SG, Binder HJ. Aldosterone and glucocorticoid receptor-specific agonists regulate ion transport in rat proximal colon. Am J Physiol. 1990;258(3 Pt 1):G492-8. doi:10.1152/ajpgi.1990.258.3.G492

21. Bazard P, Ding B, Chittam HK, et al. Aldosterone up-regulates voltage-gated potassium currents and NKCC1 protein membrane fractions. Sci Rep. 2020;10(1):1-14. 
doi:10.1038/s41598-020-72450-4

22. Ding B, Frisina RD, Zhu X, Sakai Y, Sokolowski B, Walton JP. Direct control of $\mathrm{Na}(+)-$ $\mathrm{K}(+)-2 \mathrm{Cl}(-)$-cotransport protein (NKCC1) expression with aldosterone. Am J Physiol Cell Physiol. 2014;306(1):C66-75. doi:10.1152/ajpcell.00096.2013

23. Nickerson AJ, Rajendran VM. Aldosterone up-regulates basolateral $\mathrm{Na}(+)-\mathrm{K}(+)-2 \mathrm{Cl}(-)$ cotransporter-1 to support enhanced large-conductance $\mathrm{K}(+)$ channel-mediated $\mathrm{K}(+)$ secretion in rat distal colon. FASEB J Off Publ Fed Am Soc Exp Biol. 2021;35(5):e21606. doi:10.1096/fj.202100203R

24. Martin RS, Jones WJ, Hayslett JP. Animal model to study the effect of adrenal hormones on epithelial function. Kidney Int. 1983;24(3):386-391. doi:10.1038/ki.1983.171

25. Edmonds CJ, C.J. Edmonds C.L. Willis. THE EFFECT OF DIETARY SODIUM AND POTASSIUM INTAKE ON POTASSIUM SECRETION AND KINETICS IN RAT DISTAL COLON. $J$ Physiol. 2019;53(9):1689-1699.

26. Clarke LL. A guide to Ussing chamber studies of mouse intestine. AJP Gastrointest Liver Physiol. 2009;296(6):G1151-G1166. doi:10.1152/ajpgi.90649.2008

27. Hu D, Yu Z-L, Zhang Y, et al. Bumetanide treatment during early development rescues maternal separation-induced susceptibility to stress. Sci Rep. 2017;7(1):11878. doi:10.1038/s41598-017-12183-z

28. Zhang J, Halm ST, Halm DR. Role of the BK channel (KCa1.1) during activation of electrogenic $\mathrm{K}+$ secretion in guinea pig distal colon. Am J Physiol - Gastrointest Liver Physiol. 2012;303(12):1322-1334. doi:10.1152/ajpgi.00325.2012

29. Jiang C, Kawabe H, Rotin D. The ubiquitin ligase Nedd4L regulates the $\mathrm{Na} / \mathrm{K} / 2 \mathrm{Cl}$ cotransporter NKCC1/SLC12A2 in the colon. J Biol Chem. 2017;292(8):3137-3145. doi:10.1074/jbc.M116.770065

30. Jiang G, Cobbs S, Klein JD, O'Neill WC. Aldosterone regulates the Na-K-2Cl cotransporter in vascular smooth muscle. Hypertens (Dallas, Tex 1979). 2003;41(5):1131-1135. doi:10.1161/01.HYP.0000066128.04083.CA

31. Kanthesh BM, Sandle GI, Rajendran VM. Enhanced K+ secretion in dextran sulfateinduced colitis reflects upregulation of large conductance apical $\mathrm{K}+$ channels (BK; Kcnma1). AJP Cell Physiol. 2013;305(9):C972-C980. doi:10.1152/ajpcell.00165.2013

32. Foster ES, Zimmerman TW, Hayslett JP, Binder HJ. Corticosteroid alteration of active 
electrolyte transport in rat distal colon. Am J Physiol - Gastrointest Liver Physiol. 1983;8(5):668-675. doi:10.1152/ajpgi.1983.245.5.g668

33. Field M. Intestinal ion transport and the pathophysiology of diarrhea. J Clin Invest. 2003;111(7):931-943. doi:10.1172/JCI200318326

34. Reynolds A, Parris A, Evans LA, et al. Dynamic and differential regulation of NKCC1 by calcium and cAMP in the native human colonic epithelium. J Physiol. 2007;582(2):507524. doi:10.1113/jphysiol.2007.129718

35. Bachmann $\mathrm{O}$, Wüchner $\mathrm{K}$, Rossmann $\mathrm{H}$, et al. Expression and regulation of the $\mathrm{Na}+-\mathrm{K}+-$ 2Cl- contransporter NKCC1 in the normal and CFTR-deficient murine colon. $J$ Physiol. 2003;549(2):525-536. doi:10.1113/jphysiol.2002.030205

36. Jakab RL, Collaco AM, Ameen NA. Physiological relevance of cell-specific distribution patterns of CFTR, NKCC1, NBCe1, and NHE3 along the crypt-villus axis in the intestine. Am J Physiol Gastrointest Liver Physiol. 2011;300(1):G82-98. doi:10.1152/ajpgi.00245.2010

37. Greger R. Role of Cftr in the Colon. AnnuRevPhysiol. 2000;62:467-491. doi:10.1146/annurev.physiol.62.1.467

38. Kunzelmann K, Mehta A. CFTR: A hub for kinases and crosstalk of cAMP and Ca2+. FEBS J. 2013;280(18):4417-4429. doi:10.1111/febs.12457

39. Seidler U, Blumenstein I, Kretz A, et al. A functional CFTR protein is required for mouse intestinal cAMP-, cGMP- and CA2+-dependent HCO3- secretion. J Physiol. 1997;505(2):411-423. doi:10.1111/j.1469-7793.1997.411bb.x

40. Benedetto R, Ousingsawat J, Wanitchakool P, et al. Epithelial Chloride Transport by CFTR Requires TMEM16A. Sci Rep. 2017;7(1):1-13. doi:10.1038/s41598-017-10910-0

41. Rottgen TS, Nickerson AJ, Minor EA, Stewart AB, Harold AD, Rajendran VM. Dextran sulfate sodium-induced chronic colitis attenuates $\mathrm{Ca}(2+)$-activated $\mathrm{Cl}(-)$ secretion in murine colon by downregulating TMEM16A. Am J Physiol Cell Physiol. 2018;315(1):C10-C20. doi:10.1152/ajpcell.00328.2017

42. Kunzelmann K, Centeio R, Wanitchakool P, et al. Control of Ion Transport by Tmem16a Expressed in Murine Intestine. Front Physiol. 2019;10:1262.

doi:10.3389/fphys.2019.01262

43. Ramalho AS, Lewandowska MA, Farinha CM, et al. Deletion of CFTR translation start 
site reveals functional isoforms of the protein in CF patients. Cell Physiol Biochem Int J Exp Cell Physiol Biochem Pharmacol. 2009;24(5-6):335-346. doi:10.1159/000257426

44. Pineda-Farias JB, Barragán-Iglesias P, Loeza-Alcocer E, et al. Role of anoctamin-1 and bestrophin-1 in spinal nerve ligation-induced neuropathic pain in rats. Mol Pain. 2015;11:41. doi:10.1186/s12990-015-0042-1

45. Mathialahan T, Maclennan KA, Sandle LN, Verbeke C, Sandle GI. Enhanced large intestinal potassium permeability in end-stage renal disease. $J$ Pathol. 2005;206(1):46-51. doi:10.1002/path. 1750

46. Mathialahan T, Sandle GI. Dietary potassium and laxatives as regulators of colonic potassium secretion in end-stage renal disease. Nephrol Dial Transplant. 2003;18(2):341347. doi:10.1093/ndt/18.2.341

47. Van Dinter TG, Fuerst FC, Richardson CT, et al. Stimulated active potassium secretion in a patient with colonic pseudo-obstruction: A new mechanism of secretory diarrhea. Gastroenterology. 2005;129(4):1268-1273. doi:10.1053/j.gastro.2005.07.029

48. Sandle GI, Perry MD, Mathialahan T, et al. Altered cryptal expression of luminal potassium (BK) channels in ulcerative colitis. J Pathol. 2007;212(1):66-73. doi:10.1002/path.2159

49. Sandle GI. Pathogenesis of diarrhea in ulcerative colitis: new views on an old problem. $J$ Clin Gastroenterol. 2005;39(4 Suppl 2):S49-52. doi:00004836-200504002-00004 [pii]

50. Ram P, Goyal A, Lu M, Sloan J, McElhaugh W. Use of Aldosterone Antagonist to Treat Diarrhea and Hypokalemia of Ogilvie's Syndrome. Case Rep Gastrointest Med. 2016;2016:1-3. doi:10.1155/2016/1207240

51. Lundgren O, Peregrin AT, Persson K, Kordasti S, Uhnoo I, Svensson L. Role of the enteric nervous system in the fluid and electrolyte secretion of rotavirus diarrhea. Science. 2000;287(5452):491-495. doi:10.1126/science.287.5452.491

52. Thiagarajah JR, Donowitz M, Verkman AS. Secretory diarrhoea: Mechanisms and emerging therapies. Nat Rev Gastroenterol Hepatol. 2015;12(8):446-457. doi:10.1038/nrgastro.2015.111

53. Sandle GI. Salt and water absorption in the human colon: A modern appraisal. Gut. 1998;43(2):294-299. doi:10.1136/gut.43.2.294

54. Gonzalez-Perez V, Martinez-Espinosa PL, Sala-Rabanal M, et al. Goblet cell LRRC26 
regulates BK channel activation and protects against colitis in mice. Proc Natl Acad Sci U S A. 2021;118(3). doi:10.1073/pnas.2019149118

55. Linley J, Loganathan A, Kopanati S, Sandle GI, Hunter M. Evidence that two distinct crypt cell types secrete chloride and potassium in human colon. Gut. 2014;63(3):472-479. doi:10.1136/gutjnl-2013-304695

56. Koumangoye R, Omer S, Kabeer MH, Delpire E. Novel Human NKCC1 Mutations Cause Defects in Goblet Cell Mucus Secretion and Chronic Inflammation. Cell Mol Gastroenterol Hepatol. 2020;9(2):239-255. doi:10.1016/j.jcmgh.2019.10.006

57. Yu K, Lujan R, Marmorstein A, Gabriel S, Hartzell HC. Bestrophin-2 mediates bicarbonate transport by goblet cells in mouse colon. J Clin Invest. 2010;120(5):17221735. doi:10.1172/JCI41129 


\section{TABLES:}

\begin{tabular}{|c|c|c|}
\hline $\begin{array}{l}\text { Gene Target and } \\
\text { Accession number }\end{array}$ & Primer Sequence & Amplicon length \\
\hline $\begin{array}{l}\text { NKCC1 }(\text { Slc12a2) } \\
\text { NM_001270617.1 }\end{array}$ & $\begin{array}{l}\text { F: 5'- TGGCAAGACTTCAACTCAGC - 3' } \\
\text { R: 5'- GGTATCAACAAGGTCAAACCTCC - 3' }\end{array}$ & 177 \\
\hline $\begin{array}{c}\text { BKo }(\text { Kcnmal }) \\
\text { NM_001393699.1 }\end{array}$ & $\begin{array}{l}\text { F: 5'- ATGTCTACAGTGGGTTACGG - 3' } \\
\text { R: 5'- TGGGTGGTAGTTCTTTATGG - 3' }\end{array}$ & 464 \\
\hline $\begin{array}{c}I K(\text { Kcnn4 }) \\
\text { NM_001270701.1 }\end{array}$ & $\begin{array}{l}\text { F: 5'- TGGCTGAGCACCAAGAGC - 3' } \\
\text { R: 5'- TACAGCACCCACTTGCAACC - 3' }\end{array}$ & 197 \\
\hline $\begin{array}{c}\text { CFTR (Cftr) } \\
\text { NM_031506.1 }\end{array}$ & $\begin{array}{l}\text { F: 5'- TTCTTCAGCTGGACCACACC - 3' } \\
\text { R: 5'- TGGAAGCTTGTTCCCTGTCC - 3' }\end{array}$ & 106 \\
\hline $\begin{array}{l}\text { TMEM16A (Anol) } \\
\text { NM_001107564.1 }\end{array}$ & $\begin{array}{l}\text { F: 5' - TTATGGCCCTCTGGGCTCG - 3', } \\
\text { R: 5'- CACCTCCTCTTCCTCGAAGC - 3' }\end{array}$ & 102 \\
\hline $\begin{array}{l}\beta \text {-actin }(\text { Actb) } \\
\text { NM_031144.3 }\end{array}$ & $\begin{array}{l}\text { F: 5'- AGATCAAGATCATTGCTCCTCC - 3' } \\
\text { R: 5'- AGTAACAGTCCGCCTAGAAGC - 3' }\end{array}$ & 165 \\
\hline
\end{tabular}

Table 1: Primer sequences used in qRT-PCR analysis. 


\begin{tabular}{|c|c|c|c|}
\hline Target & Host Species & Supplier & Dilution (Application) \\
\hline NKCC1 & Rabbit & Cell Signaling & $\begin{array}{c}1: 500(\mathrm{WB}) \\
1: 500(\mathrm{IF})\end{array}$ \\
\hline$B K \alpha$ & Rabbit & Alomone & $1: 250(\mathrm{WB})$ \\
\hline$I K$ & Rabbit & $\begin{array}{c}\text { Gift from Dr. Michael } \\
\text { Kashgarian }\end{array}$ & $1: 3,000(\mathrm{WB})$ \\
\hline CFTR & Rabbit & Alomone & $1: 250(\mathrm{WB})$ \\
\hline$T M E M 16 A$ & Rabbit & Alomone & $1: 250(\mathrm{WB})$ \\
\hline$\beta$-actin & $\begin{array}{c}\text { Mouse } \\
\text { (HRP-conjugated) }\end{array}$ & SCBT & $1: 1,000(\mathrm{WB})$ \\
\hline
\end{tabular}

Table 2: Primary antibody product and usage information. 


\begin{tabular}{|c|c|c|c|c|c|c|c|}
\hline & \multicolumn{4}{|c|}{ 复的 ${ }^{+}$Fluxes } & \multirow[b]{2}{*}{$n$} & \multirow[b]{2}{*}{$\begin{array}{c}\mathbf{I}_{\mathbf{S C}} \\
\left(\mu \mathrm{A} / \mathrm{cm}^{2}\right)\end{array}$} & \multirow[b]{2}{*}{$\begin{array}{c}\mathbf{G}_{\mathbf{T E}} \\
\left(\mathrm{mS} / \mathrm{cm}^{2}\right)\end{array}$} \\
\hline Condition & $n$ & $\begin{array}{c}\mathbf{J}_{\mathbf{M}-\mathbf{S}} \\
\left(\mu \mathrm{Eq} / \mathrm{cm}^{2} \cdot \mathrm{hr}\right)\end{array}$ & $\begin{array}{c}\mathbf{J}_{\mathbf{S}-\mathbf{M}} \\
\left(\mu \mathrm{Eq} / \mathrm{cm}^{2} \cdot \mathrm{hr}\right)\end{array}$ & $\begin{array}{c}\mathbf{J}_{\mathrm{Net}} \\
\left(\mu \mathrm{Eq} / \mathrm{cm}^{2} \cdot \mathrm{hr}\right)\end{array}$ & & & \\
\hline Normal & 6 & $0.6 \pm 0.1$ & $1.1 \pm 0.1$ & $-0.4 \pm 0.1$ & 12 & $54.2 \pm 9.2$ & $13.4 \pm 1.0$ \\
\hline "Aldo" & 8 & $0.8 \pm 0.1$ & $3.0 \pm 0.5$ & $-2.1 \pm 0.4$ & 16 & $88.0 \pm 8.2$ & $17.3 \pm 1.1$ \\
\hline$p$ & & 0.2623 & $0.0071^{*}$ & $0.0033^{*}$ & & $<0.0111^{*}$ & $<0.0191^{*}$ \\
\hline
\end{tabular}

Table 3: ${ }^{86} \mathrm{Rb}^{+}$fluxes in normal and dietary $\mathrm{Na}^{+}$-depleted (“aldo") rat proximal colon. Unidirectional ${ }^{86} \mathrm{Rb}^{+}$fluxes were measured across seromuscular-stripped rat proximal colon under voltage-clamped conditions. Mucosal-to-serosal (M-S) and serosal-to-mucosal (S-M) fluxes were measured over a 15-minute period. Net fluxes were calculated by subtracting S-M from M-S fluxes using tissues pairs matched by conductance, as described in Methods. Flux values are given as mean \pm SEM. $I_{S C}$ and $\mathrm{G}_{\mathrm{TE}}$ values represent the averages recorded over the 15 -minute flux period and are given as mean \pm SEM. ${ }^{*}$ Indicates significance compared to control (untreated), as determined by unpaired Student's t-test. 
A

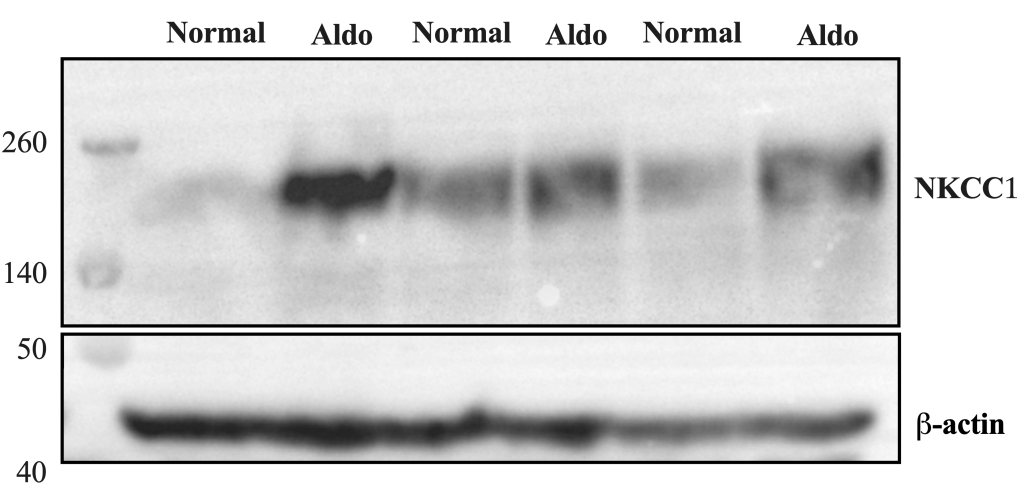

B

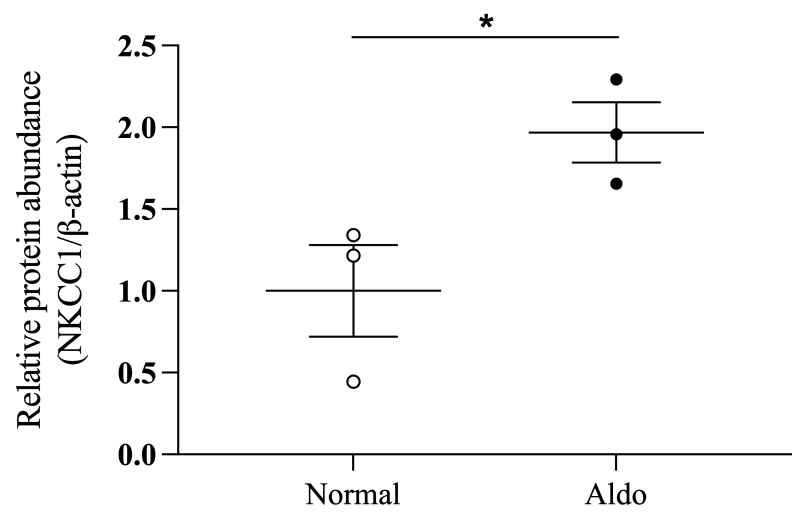

C

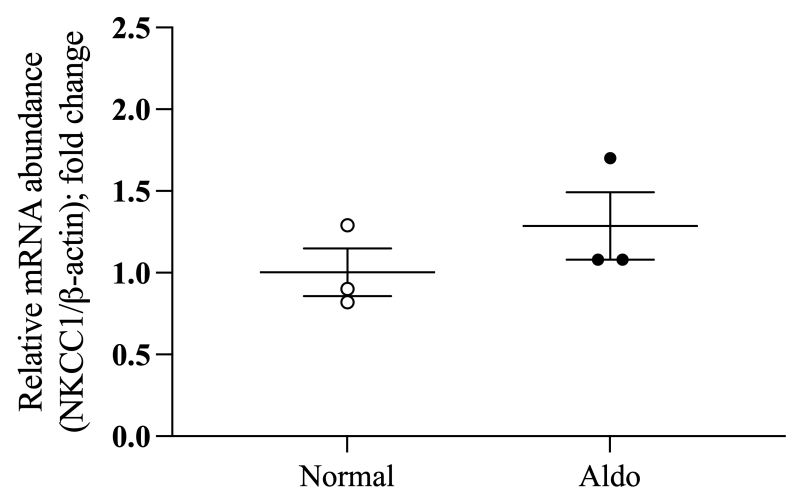

Figure 1 -Dietary $\mathrm{Na}^{+}$depletion (aldosterone) enhances NKCC1 protein, but not mRNA expression in rat proximal colon. $A$ : Western blot performed using mucosal homogenates from 
normal and $\mathrm{Na}^{+}$-depleted ("aldo") rat proximal colon. Standard molecular weights and primary antibody targets are indicated to the left and right of the blot images, respectively. $B$ : NKCC1 band intensity was quantified using densitometry analysis in ImageJ and normalized to actin band intensity as a loading control for each sample ( $n=3$ separate animals). $C$ : qRT-PCR analysis of NKCC1-specific mRNA transcript abundance between normal and aldo rat proximal colon. Data are represented as fold change relative to control (Normal). Lines and error bars represent mean \pm SEM. ${ }^{*}<0.05$ compared with control (Normal) using unpaired Student's t-test. 


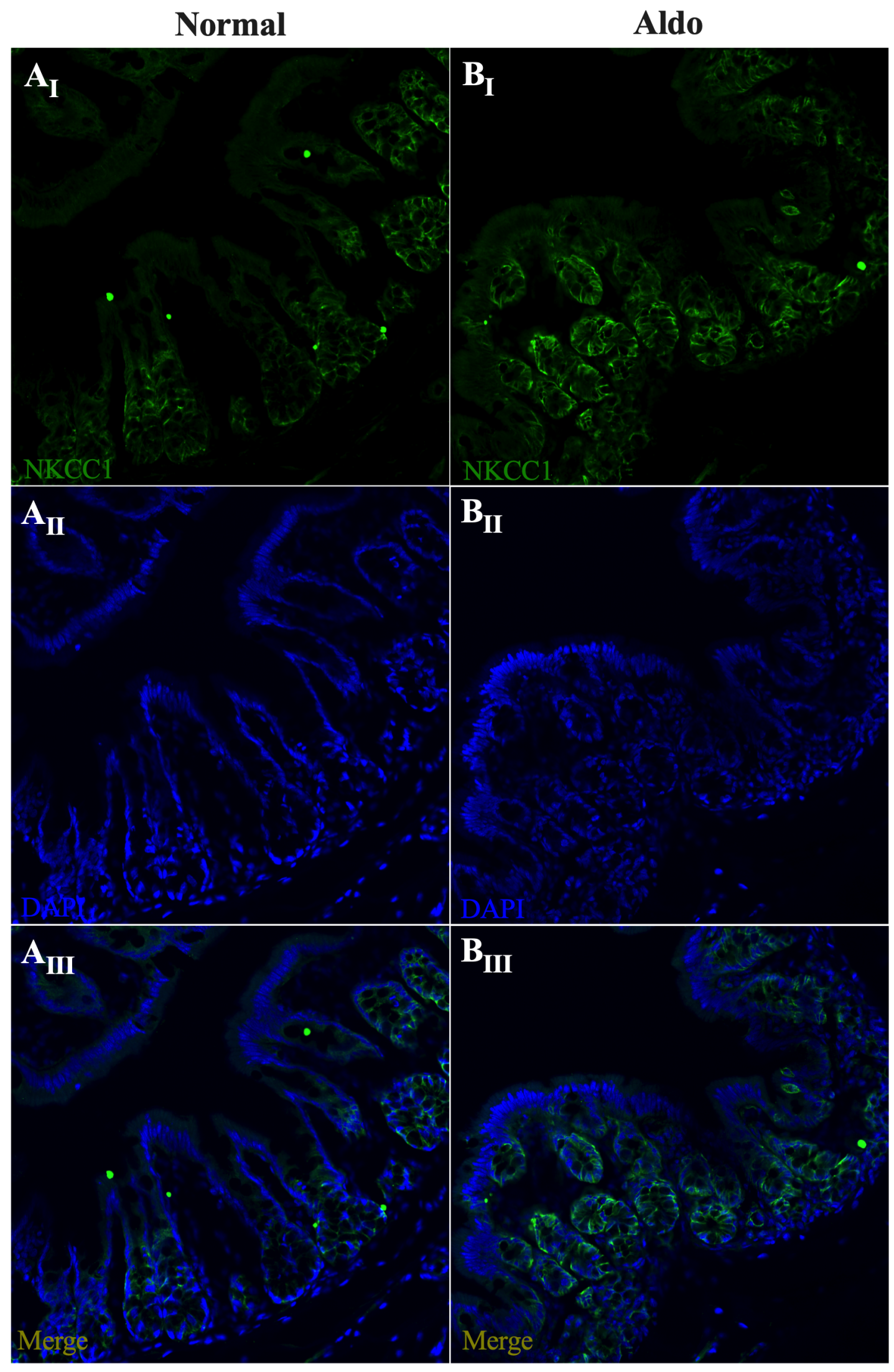


Figure 2 - Immunofluorescent labelling of NKCC1 is enhanced by dietary $\mathrm{Na}^{+}$depletion in rat proximal colon. $\mathrm{NKCC} 1$-specific labelling (green) in normal $(A)$ and $\mathrm{Na}^{+}$-depleted (aldo) $(B)$ proximal colon sections. Nuclei are labelled with DAPI (blue). Images were captured at 20x magnification. Scale bar $=50 \mu \mathrm{m}$.

A

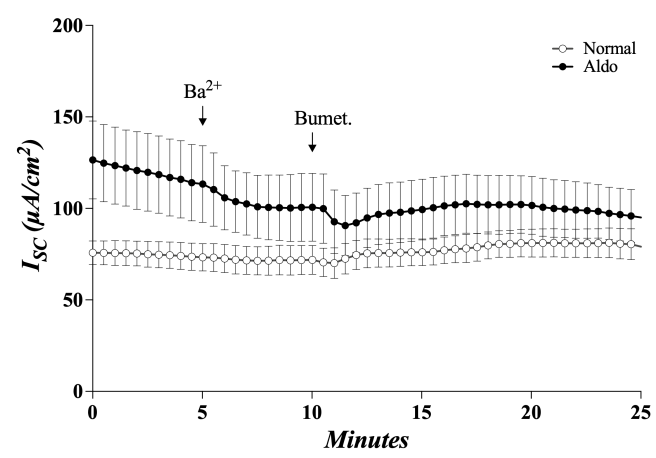

B Trans-epithelial conductance $\left(\mathrm{G}_{\mathrm{TE}}\right)$

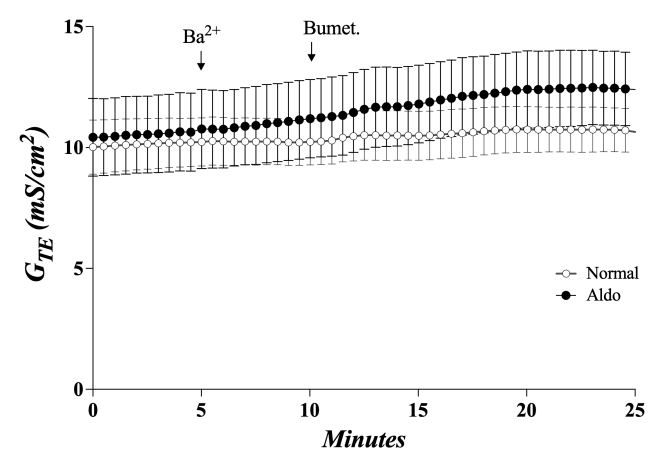

C

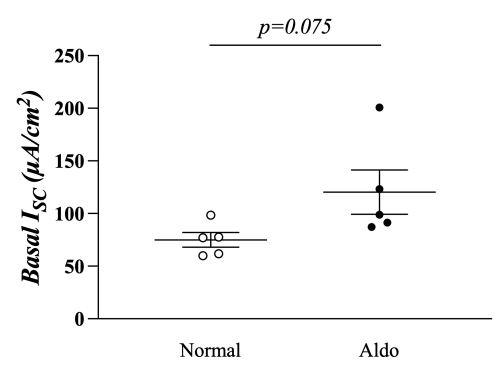

D $\quad \mathrm{Ba}^{2+}$-sensitive $\mathrm{I}_{\mathrm{SC}}$

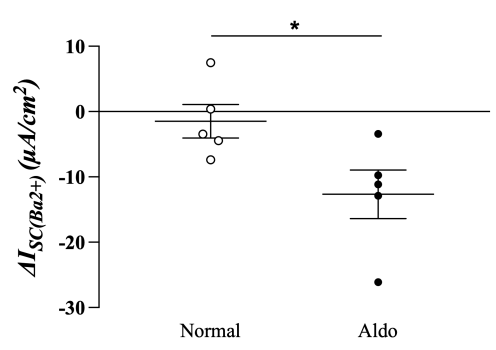

$\mathbf{E}$

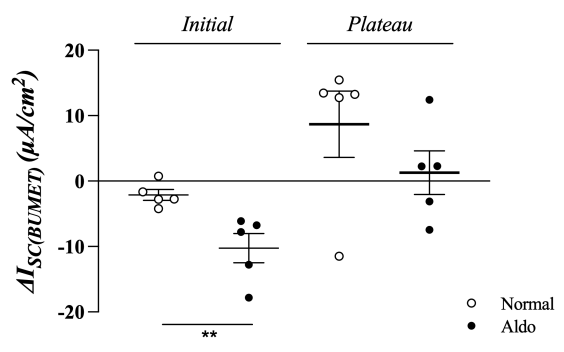

Figure 3 - Dietary $\mathrm{Na}^{+}$depletion does not enhance $\mathrm{Ba}^{2+}$ - or bumetanide-sensitive electrogenic $\mathrm{K}^{+}$secretion in rat proximal colon. Short-circuit current $\left(\mathrm{I}_{\mathrm{SC}}\right)(A)$ and trans-epithelial conductance $\left(\mathrm{G}_{\mathrm{TE}}\right)(B)$ recordings from normal (open circles) and $\mathbf{N a}^{+}$-depleted (aldo) (closed circles) proximal colon treated sequentially with mucosal $3 \mathrm{mM} \mathrm{Ba}^{2+}$ and serosal $200 \mu \mathrm{M}$ bumetanide (BUMET) where indicated. Basal $(C), \mathrm{Ba}^{2+}$ sensitive $(D)$ and bumetanide-sensitive (E) $\mathrm{I}_{\mathrm{SC}}$ from normal and aldo proximal colon. $\mathrm{Ba}^{2+}$-sensitive and bumetanide-sensitive $\mathrm{I}_{\mathrm{SC}}$ values 
were calculated as the change in $\mathrm{I}_{\mathrm{SC}}\left(\Delta \mathrm{I}_{\mathrm{SC}}\right)$ from before and after the addition of drug to the chamber bath. Lines and error bars represent mean \pm SEM. $n=5$ tissues from 3 separate animals. ${ }^{*} p<0.05$ and ${ }^{* *} p<0.01$ compared with control (Normal) using unpaired Student’s t-test.

A

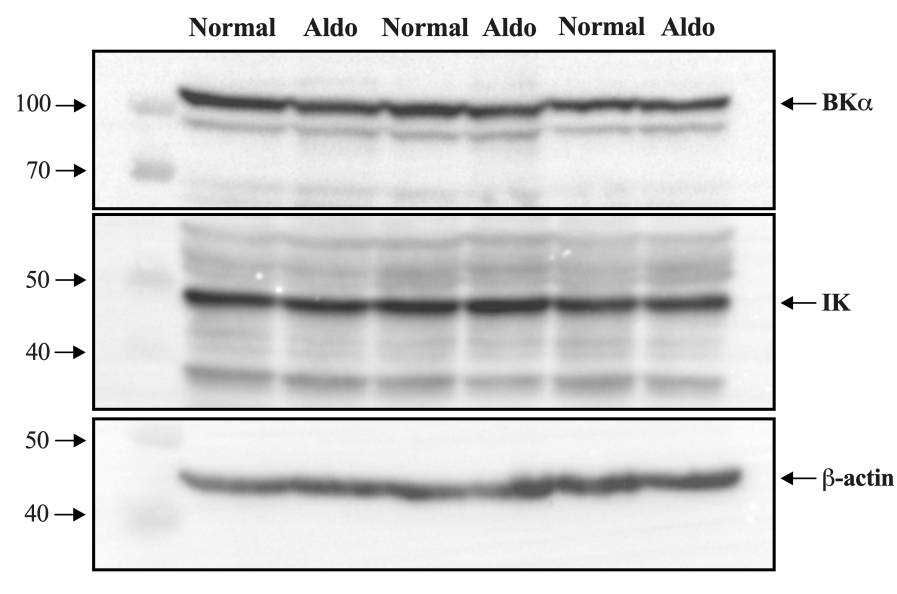

B

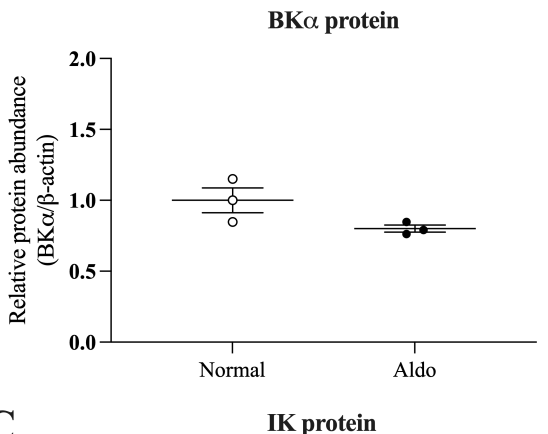

C

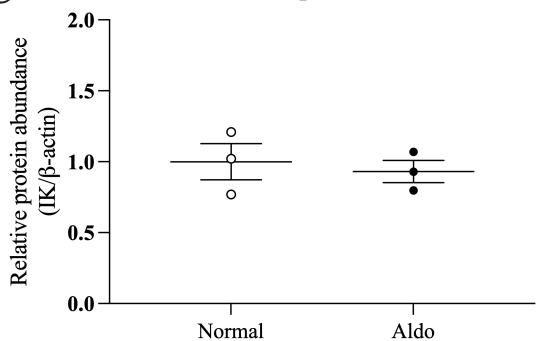

D

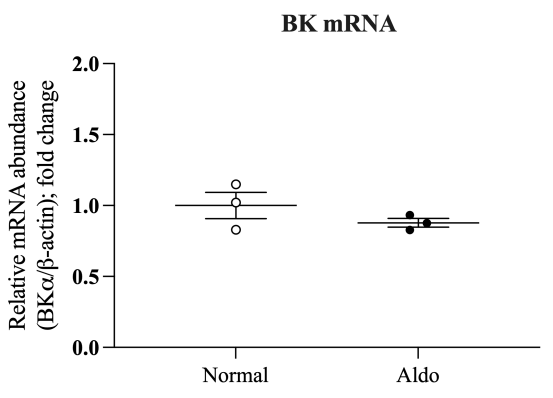

$\mathbf{E}$

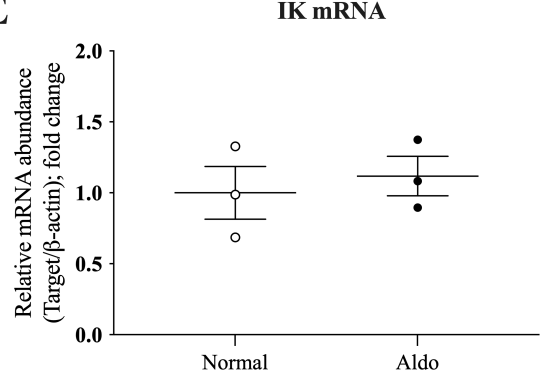

Figure 4 - Dietary $\mathrm{Na}^{+}$depletion does not enhance BK or IK channel expression in rat proximal colon. $A$ : Western blots performed using mucosal homogenates from normal and $\mathrm{Na}^{+}$depleted (aldo) rat proximal colon tissues. Standard molecular weights and primary antibody targets are indicated to the left and right of the blot images, respectively. BK $\alpha(B)$ and IK $(C)$ band intensities were quantified using densitometry analysis in Image $J$ and normalized to actin band 
intensity as a loading control for each sample ( $n=3$ separate animals). qRT-PCR analysis of BKspecific $(D)$ and IK-specific $(E)$ mRNA transcript abundance between normal and aldo rat proximal colon. Data are represented as fold change relative to control (Normal). Lines and error bars represent mean $\pm \mathrm{SEM}$.

A

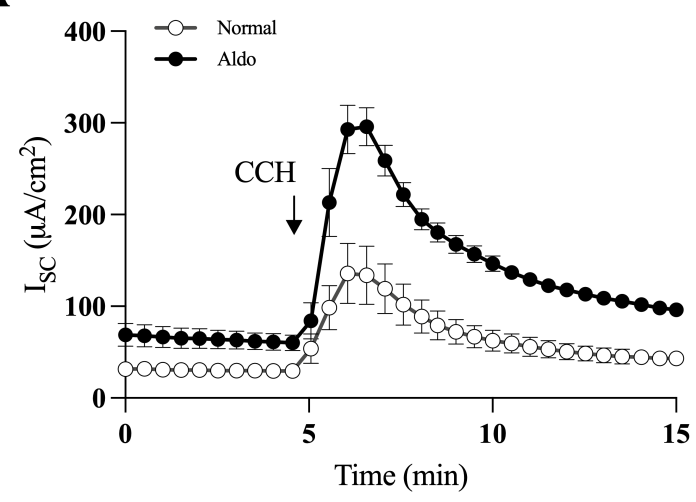

B

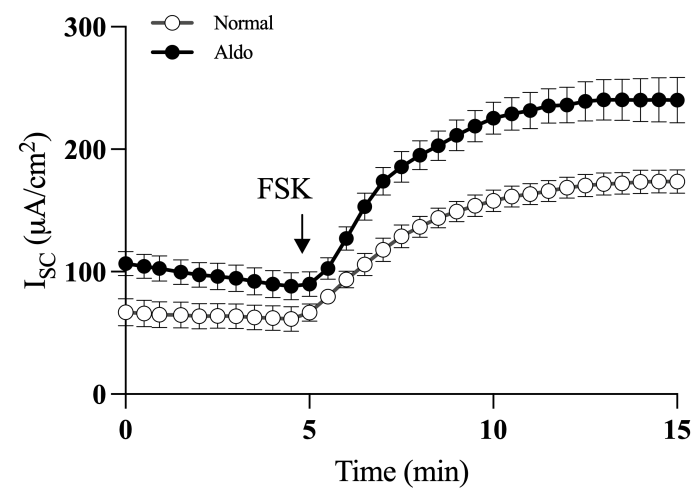

C

Basal $\mathrm{I}_{\mathrm{SC}}$

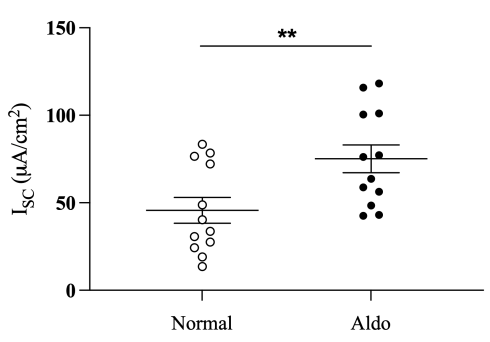

D

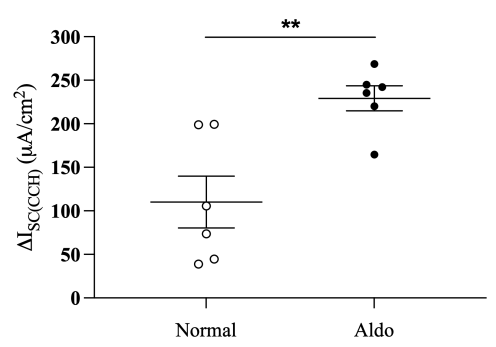

E cAMP-stimulated I $\mathrm{I}_{\mathrm{SC}}$

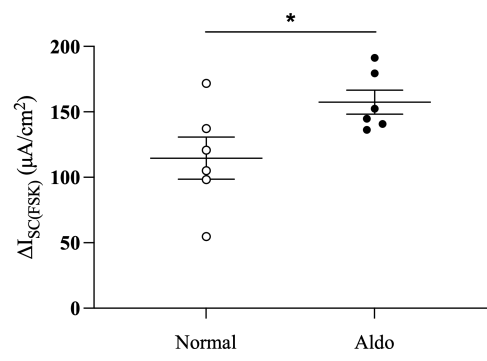

Figure 5 - Dietary $\mathrm{Na}^{+}$depletion enhances $\mathrm{Ca}^{2+}$ - and cAMP-stimulated $\mathrm{Cl}^{-}$secretion in rat proximal colon. Short-circuit current $\left(\mathrm{I}_{\mathrm{SC}}\right)$ recordings from normal (open circles) and $\mathrm{Na}^{+}$depleted (aldo) (closed circles) proximal colonic mucosae treated with serosal $100 \mu \mathrm{M}$ carbachol $(\mathrm{CCH})(A)$ or serosal $10 \mu \mathrm{M}$ forskolin $(\mathrm{FSK})$ where indicated. Basal $(C)$, carbachol $\left(\mathrm{Ca}^{2+}\right)$ stimulated $(D)$ and forskolin (cAMP)-stimulated $\mathrm{I}_{\mathrm{SC}}$ from normal and aldo proximal colon. $\Delta \mathrm{I}_{\mathrm{SC}}$ values were calculated as the change from baseline to post-drug peak $\mathrm{I}_{\mathrm{SC}}$ for carbachol and from 
baseline to post-drug plateau $\mathrm{I}_{\mathrm{SC}}$ for forskolin. Lines and error bars represent mean \pm SEM. $n=6$ tissues from 3 separate animals for carbachol and forskolin experiments, totaling 12 tissues from 6 separate animals for basal ISC measurements. ${ }^{*} p<0.05$ and ${ }^{* *} p<0.01$ compared with control (Normal) using unpaired Student's t-test. 

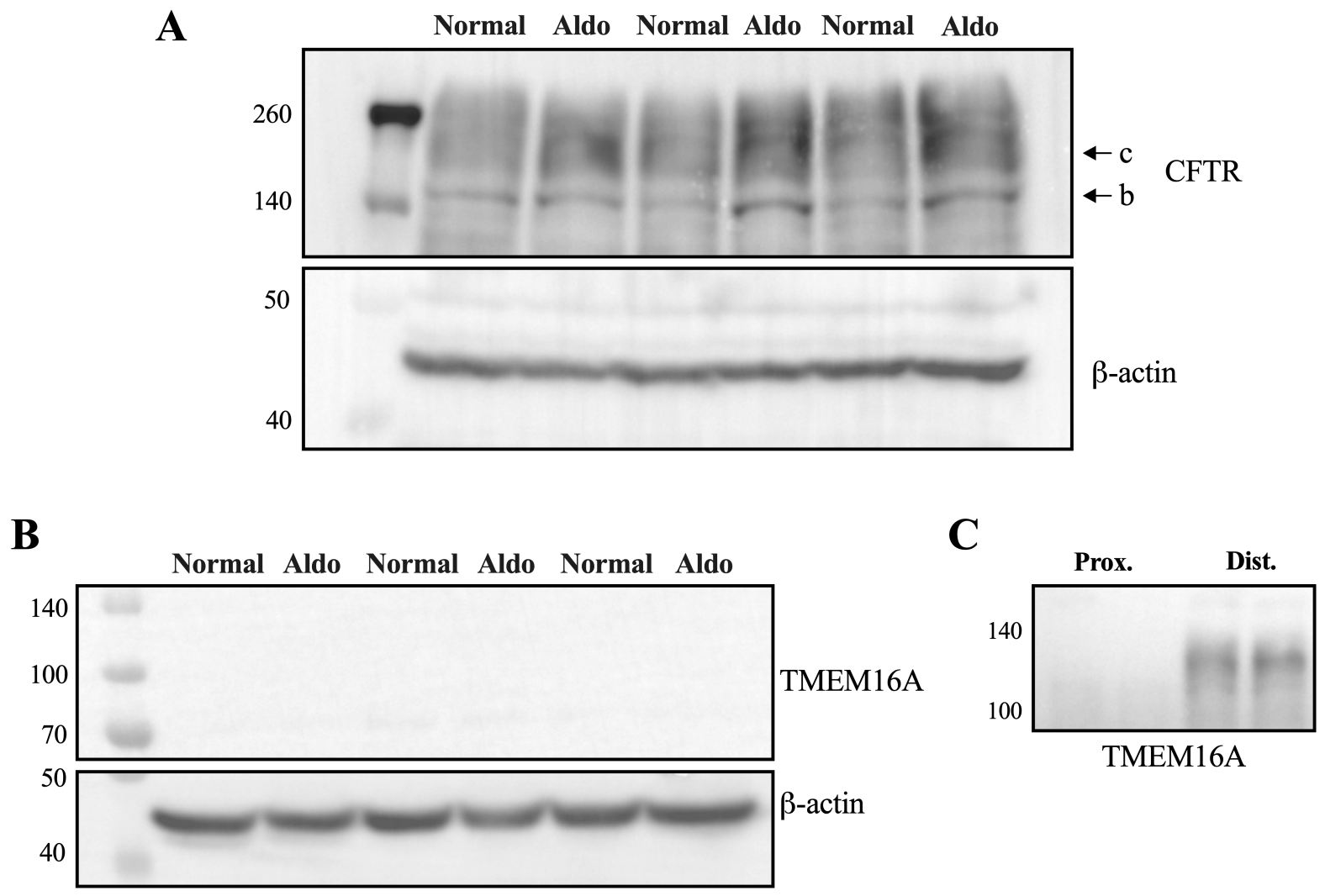

D

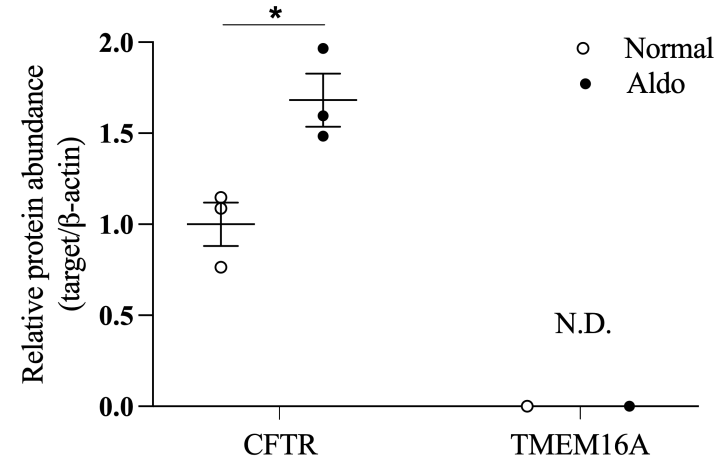

$\mathbf{E}$

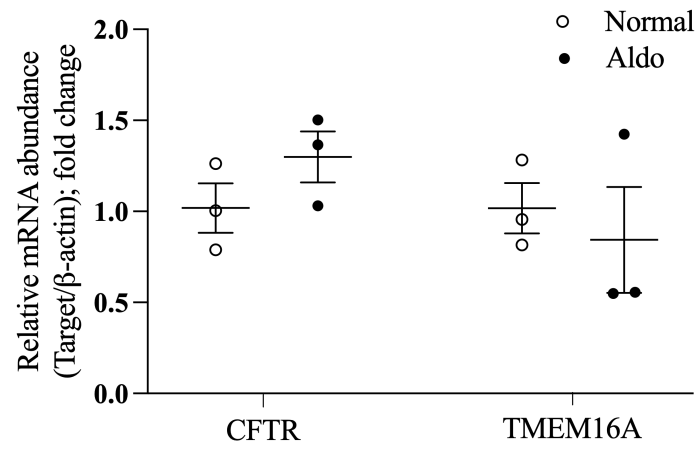

Figure 6 - Dietary $\mathrm{Na}^{+}$depletion enhances $\mathrm{CFTR} \mathrm{Cl}^{-}$channel expression in rat proximal colon. $A-B$ : Western blots performed using mucosal homogenates from 3 normal and $3 \mathrm{Na}^{+}-$ depleted (aldo) rat proximal colons. Standard molecular weights and primary antibody targets are 
indicated to the left and right of the blot images, respectively. $C$ : Representative blot showing TMEM16A labelling in normal distal, but not proximal colon homogenates as a positive control for antibody specificity. $D$ : Band intensities were quantified using densitometry analysis in ImageJ and normalized to actin band intensity as a loading control for each sample ( $n=3$ separate animals). E: qRT-PCR analysis of CFTR- and TMEM16A-specific mRNA transcript abundance between 3 normal and 3 aldo rat proximal colon. Data are represented as fold change relative to control (Normal). Lines and error bars represent mean \pm SEM. ${ }^{*} p<0.05$ compared with control (Normal) using unpaired Student's t-test. 


\section{CHAPTER 6: GENERAL DISCUSSION}

The work presented in this dissertation sheds new light on several regulatory mechanisms that govern colonic epithelial ion transport. Furthering our understanding of these mechanisms may prove useful in developing therapeutic strategies to treat conditions related to aberrant colonic ion transport, such as IBS-D or IBD, as managing electrolyte and fluid absorption is a primary goal in alleviating problematic symptoms associated with these conditions $(1,2)$. Regulation of colonic electrolyte transport is a vastly complex matter, as discussed in Chapter 1 . The studies undertaken in this project have been centered around the characterization of two major, but sperate control mechanisms in the regulation of colonic electrolyte transport: regulation by submucosal neurons of the enteric nervous system, and regulation by the mineralocorticoid hormone, aldosterone. Associated with each of these sub-projects is its own set of implications for disease treatments and opportunities to expand upon the work that we have done. As such, each will be discussed separately.

\section{1 - A novel role for $K_{V} 7$ channels in controlling ENS-dependent epithelial transport.}

The enteric nervous system (ENS) is a powerful regulator of epithelial transport throughout the G.I. tract, including in the colon $(3,4)$. It is capable of sensing chemical and mechanical stimuli and generating a reflexive response completely independent of external inputs, although physiologically, many points of connection exist between the ENS and other neural networks (5). In general, input to the epithelium from the ENS triggers a secretory response involving enhanced secretion of $\mathrm{Cl}^{-}$and reduced absorption of $\mathrm{Na}^{+}$for the purpose of driving water secretion to the

lumen (via osmosis) and flushing out potentially harmful substances (6,7). Unfortunately, this 
process often proceeds inappropriately in response to everyday stress and sometimes without any provocation, leading to pathological conditions such as IBS-D. Many pharmacotherapies target the ENS by suppressing neuronal activity (8), but existing treatments are commonly associated with adverse side effects. Our studies have identified a novel target $\left(\mathrm{K}_{\mathrm{V}} 7\right.$ channels) in developing treatment strategies for ENS-driven pathologies such as IBS-D.

Voltage-gated $\mathrm{K}_{\mathrm{V}} 7$ channels ( $K C N Q$ gene family) have been extensively characterized in central and peripheral neural populations, as well as in other non-neuronal cell types $(9,10,11)$. These channels open at relatively negative (hyperpolarized) membrane potentials to permit $\mathrm{K}^{+}$ efflux from the cell (12). This suppresses excitability by hyperpolarizing the membrane, making the generation of action potentials (in neurons) more unlikely. Consequently, $\mathrm{K}_{\mathrm{V}} 7$-activating drugs have been used for the treatment of chronic pain and epilepsy (13) - both of which are related to excessive neuronal firing. However, it was not previously known whether $\mathrm{K}_{\mathrm{V}} 7$ channels were expressed by, or had a functional role within, neurons of the ENS.

\subsection{1 - Brief summary of findings.}

We have demonstrated that at least two $\mathrm{K}_{\mathrm{V}} 7$ channel subtypes $\left(\mathrm{K}_{\mathrm{V}} 7.2 / 3\right)$ are expressed by neurons of the submucosal plexus (Chapter 2, Fig. 10; Chapter 3 Fig. 1), and that their activation suppresses $\mathrm{Cl}^{-}$secretion (see Chapter 2) and enhances basal $\mathrm{Na}^{+}$absorption (see Chapter 3) by dampening stimulatory ENS input to the colonic epithelium. Each of these outcomes would, in theory, lead to an increase in the rate of water absorption by enhancing the osmotic gradient across the mucosal barrier (14). This, coupled with other reports of $\mathrm{K}_{\mathrm{V}} 7$ channel activators suppressing contractility (11) and afferent sensory neuron activity (15), make this class of drugs an attractive target for treatment of IBS-D. Furthermore, $\mathrm{K}_{\mathrm{V}} 7$ activation appears to modulate epithelial transport 
specifically in the colon (Chapter 3, Fig. 7), potentially minimizing any widespread or "off-target" effects that may arise with drugs acting throughout the GI tract.

The findings from these two studies build upon our current understanding of ENS function. Specifically, the expression of $\mathrm{K}_{\mathrm{V}} 7$ channel subtypes in submucosal neurons had not yet been demonstrated, nor had the effects of Flupirtine or other $\mathrm{K}_{\mathrm{V}} 7$ activators been evaluated with respect to epithelial ion transport. The novel observation that ENS-localized $\mathrm{K}_{\mathrm{V}} 7$ activation inhibits neurogenic secretion fits nicely with the channels' known role in suppressing neural activity $(10,16,17)$. In terms of the resultant effects on epithelial $\mathrm{Cl}^{-}$and $\mathrm{Na}^{+}$transport, the results are in line with published literature that has characterized the regulation of transport proteins and channels in response to ENS-derived neurotransmitters $(3,18,19,20)$.

While we did not fully characterize the epithelial intracellular signaling events in response to neuronal $\mathrm{K}_{\mathrm{V}} 7$ activation, it stands to reason that such events would proceed as has been described by others. Namely, $\mathrm{Ca}^{2+}$ and/or cAMP second messenger systems would likely diminish as a result of reduced activation of basolateral receptors, causing a fall in secretion and a rise in absorption. Thus, the novel aspect of these studies is that we have identified a "new" upstream target in the suppression of neurogenic secretion in the colon, and not an entirely novel ion transport mechanism altogether. This is perhaps very advantageous with respect to therapeutic development because many of the downstream mechanistic details are already well-established. In addition, $\mathrm{K}_{\mathrm{V}} 7$ activating drugs are known to be well-tolerated by patients $(13,21)$.

\subsection{2 - Remaining questions and future directions.}

Does inhibition of ENS-localized $K_{V} 7$ channels lead to increased water absorption the colon as a result of the induced changes in ion transport? 
Transport of solutes allows for the movement of water in biological systems, as water cannot be transported directly. We predict that decreased $\mathrm{Cl}^{-}$secretion and enhanced $\mathrm{Na}^{+}$ absorption together would likely drive an increase in overall water absorption in response to ENSlocalized $\mathrm{K}_{\mathrm{V}} 7$ activation, but we have not yet demonstrated a change in water absorption directly. Thus, the most direct and logical next step in determining the efficacy of $\mathrm{K}_{\mathrm{V}} 7$ activators in treating diarrhea is to determine the effects on water transport in the colon. This can be done through in vivo perfusion studies using either tritiated polyethylene glycol $\left({ }^{3} \mathrm{H}-\mathrm{PEG}\right)$ or non-absorbable fluorescent solutes (e.g., FITC-dextran) as described previously $(22,23)$. These experiments would involve canulating the large intestine and passing solution through at a slow rate, while collecting from the distal end to calculate change in solute concentration (and thus change in water content). For a less invasive approach, stool wet/dry weight could also easily be measured in animals treated with flupirtine or vehicle to observe the effects in a truly unadulterated context.

IBS-D is commonly associated with stress and symptoms may precipitate in stressful situations (24). $\mathrm{K}_{\mathrm{V}} 7$ activators may also alleviate stress-induced $\mathrm{Cl}^{-}$secretion, as much of this is driven by ENS activity. Because these drugs generally depress neuronal activity, we predict that a secretory response to stressful stimuli would be blunted in animals treated with $\mathrm{K}_{\mathrm{V}} 7$ activators. A combination of behavioral assays to evaluate stress sensitivity (e.g., social interaction test or chronic mild stress models) (25), as well as ex vivo and in vivo experiments to determine electrolyte and water transport will provide insight into the utility of such drugs in alleviating symptoms of IBS-D, and in particular, stress-related IBS-D. 


\section{Are $K_{V} 7$ channels expressed in specific populations of ENS neurons?}

We have shown that the effects of flupirtine are apparently specific to the colon, as the ileum was not responsive to treatment in the Ussing chamber experiments detailed in Chapter 2. Within the colon, there are at least two major populations of secretomotor neurons: cholinergic and VIP-ergic $(26,27)$. There are also nitrergic (nNOS) neurons, although they are far fewer in number (27). RNA scope analysis, or simple dual labelling via immunohistochemistry can be used

to determine whether $\mathrm{K}_{\mathrm{V}} 7$ channels are expressed within any of these sub-populations by identifying cells expressing $K C N Q$ genes in conjunction with Choline acetyltransferase (cholinergic neuron marker), VIP or neuronal nitric oxide synthase (nNOS, a nitrergic neuronal marker). It is also possible that other non-secretomotor populations express $\mathrm{K}_{\mathrm{V}} 7$ channels as well, including interneurons, sensory afferents or even glia. RNA scope may provide the starting point for identifying the relevant subpopulations, while immunofluorescent labelling can be used to confirm these findings in native tissue. Characterizing the expression pattern of $\mathrm{K}_{\mathrm{V}} 7$ channel subtypes in ENS neurons will inform future initiatives in therapeutic development by revealing the neurochemical and histological landscape in which $\mathrm{K}_{\mathrm{V}} 7$ activating drugs operate within the gut.

\section{Are these novel effects of $K_{V} 7$ channel activators translatable to the human condition?}

Many model systems for studying human colonic ion transport exist. However, the majority of these rely on culture of epithelial cells or tissues, and thus lack innervation by an ENS or ENS-like neural structure. Only recently have there been reports of co-culture systems using human tissue-derived organoid monolayers and neuronal progenitor cells together in culture $(28,29)$. Even so, many limitations likely exist in this context, as development of both the 
epithelium and the nervous tissue is driven artificially. Therefore, we propose that anatomical and functional characterization be performed using intact human tissue derived from surgical resection. Often, in patients with adenocarcinoma, clean margins of tissue must be excised when removing tumors. These margins provide a unique opportunity to examine ion transport or probe the neural networks using the techniques described above. Doing so will provide the all-important next step in the development of this drug class in treating human pathologies.

Another undertaking aside from wet lab experiments that will be useful in downstream applications is examination of patient records for individuals prescribed flupirtine in the clinic. Flupirtine was used for many years in the treatment of chronic pain and epilepsy, and patient data is likely still available. To our knowledge, there has not been an analysis of G.I. symptom reporting (i.e., incidence of diarrhea) in patients with pre-existing diarrheal conditions (i.e., IBS-D). Should there be a link between clinical flupirtine usage and alterations in G.I. symptoms, this will provide invaluable insight into drug development. If a positive relationship does indeed exist in the patient record, this may very well expedite the timeline of bringing $\mathrm{K}_{\mathrm{V}} 7$ activators to market for treatment of IBS-D.

6.2 - Characterization of the role of NKCC1 in aldosterone-stimulated $\mathrm{K}^{+}$secretion in colonic epithelium.

Much attention has been directed toward delineating $\mathrm{K}^{+}$transport pathways in the colon because the colon plays a supportive, but critical role in maintaining body $\mathrm{K}^{+}$homeostasis $(30,31)$. The combined efforts of the kidney and colon balance dietary $\mathrm{K}^{+}$intake to $\mathrm{K}^{+}$excretion/secretion. Because even small fluctuations in extracellular (i.e., blood) $\mathrm{K}^{+}$concentrations can have life threatening consequences, $\mathrm{K}^{+}$transport systems in these tissues have been studied extensively. 
Aldosterone is known to play a central role in stimulating $\mathrm{K}^{+}$secretion and exerts its effects primarily by influencing the transcription of genes related to electrolyte transport, including the channels and transporters themselves or regulatory factors (kinases, ubiquitin ligases, etc.) $(32,33,34,35)$. Within the colon, isotopic flux and shot-circuit current $\left(\mathrm{I}_{\mathrm{SC}}\right)$ experiments have revealed that, as in the kidney, increased circulating aldosterone triggers both short- and long-term stimulation of pathways involved in $\mathrm{K}^{+}$secretion. However, some underlying details had not yet been elucidated.

\subsection{1 - Brief summary of findings.}

Past studies had focused primarily on identifying the aldosterone-regulated channel proteins that facilitate $\mathrm{K}^{+}$efflux across the apical membranes of transporting epithelial cells (e.g., $\mathrm{BK} \alpha)(32,36)$. We have built upon this by demonstrating for the first time that 1) aldosterone transcriptionally regulates a key basolateral $\mathrm{K}^{+}$co-transporter, $\mathrm{NKCC} 1$, specifically to support enhanced and sustained BK-mediated $\mathrm{K}^{+}$secretion (see Chapter 4), and 2) that there is segmental heterogeneity with respect to this phenomenon, as this effect is only seen in the distal region of the colon (see Chapter 5).

Aldosterone was already known to regulate various transporters and channels, including $\mathrm{NKCC} 1$ and $\mathrm{BKa}$, via mechanisms other than direct transcriptional regulation. For example, $\mathrm{NKCC} 1$ is regulated by controlling the number of functional transporters that are active and localized to the basolateral membrane. Aldosterone inhibits a ubiquitin ligase called Nedd4L which, in turn, prevents the targeting of $\mathrm{NKCC} 1$ for degradation via the proteasomal pathway $(34,37)$, thus increasing the pool of available NKCC1 protein. Aldosterone had also been shown to activate protein kinase $\mathrm{C}$ directly (38), which may well have downstream control over various 
transport pathways. Here, we demonstrate transcriptional control of aldosterone over NKCC1 in the distal colon, and perhaps more importantly, the specificity of increased NKCC1 function to the $\mathrm{K}^{+}$transport pathway. This is important because in many tissues, including in the colon under normal physiologic conditions, $\mathrm{NKCCl}$ is considered to support $\mathrm{Cl}^{-}$secretion primarily (39). Thus, we have identified a new context in which NKCC1 function may be considered.

Interestingly, $\mathrm{NKCC} 1$ expression was also increased by aldosterone in the proximal colon, although not at the mRNA transcript level. Here, NKCC1-dependent $\mathrm{K}^{+}$secretion was also not apparently increased, whereas $\mathrm{Cl}^{-}$secretion was. This suggests that there may be an inherent difference in the regional response to circulating aldosterone. One possible explanation for this is that aldosterone may signal through the glucocorticoid receptor in the proximal colon, resulting in increased NKCC1 protein abundance through non-transcriptional regulation, such as alterations in protein turnover, etc. There have been other reports of aldosterone possibly signaling through both mineralocorticoid and glucocorticoid receptors in the colon, each with its own set of outcomes regarding epithelial transport (40). Supporting this possibly is the observation that apical membrane $\mathrm{K}^{+}$channel expression is not affected by aldosterone in the proximal colon, nor is the amount of $\mathrm{Ba}^{2+}$-sensitive $\mathrm{I}_{\mathrm{SC}}$, which should reflect the basal activity of any/all apical membrane $\mathrm{K}^{+}$channel(s) expressed here. These characteristics are in sharp contrast to those of the distal colon, where mineralocorticoid signaling predominates. Throughout the small intestine - a tissue which cannot signal through the mineralocorticoid receptor - NKCC1 primarily supports $\mathrm{Cl}^{-}$ secretion as well.

In spite of this, data from our lab and others have repeatedly shown increased overall rates of luminal $\mathrm{K}^{+}$flux as measured by radioisotope tracer experiments. These two findings - that net $\mathrm{K}^{+}\left({ }^{86} \mathrm{Rb}\right.$ ) flux is enhanced, but that luminal $\mathrm{Ba}^{2+}$ (or any selective $\mathrm{K}^{+}$channel blocker) does not 
seem to inhibit either isotope flux or $\mathrm{I}_{\mathrm{SC}}$, seem at first to be immiscible observations. However, a recent study reported that $\mathrm{K}^{+}$secretion is largely confined to the goblet cells of the colon (41) cell type in which $\mathrm{NKCC} 1$ is also highly expressed. It may be that in animals with elevated circulating aldosterone, goblet cells take up a greater amount of $\mathrm{K}^{+}$across the basolateral membrane due to the increase in NKCC1. Subsequently, this $\mathrm{K}^{+}$may be released to the luminal via degranulation (the process by which goblet cells secrete mucus), rather than via diffusion through an apical membrane channel protein. Experiments to determine whether this actually occurs will be discussed below.

Colonic $\mathrm{K}^{+}$secretion is only about $5 \%$ of that of the kidney (30). However, in certain pathological conditions, the capacity for $\mathrm{K}^{+}$secretion becomes very important. In patients with renal failure, the colon serves as a secondary outlet for $\mathrm{K}^{+}$from the body and can prevent lifethreatening hyperkalemia $(42,43)$. Conversely, some diarrheal diseases such as colonic pseudoobstruction (Ogilvie's syndrome), ulcerative colitis, and $V$. cholera infection are characterized by excessive $\mathrm{K}^{+}$secretion in the colon $(44,45,46,47)$. In at least one patient with pseudo-obstruction, the use of spironolactone (a mineralocorticoid receptor antagonist) attenuated excessive $\mathrm{K}^{+}$ secretion and stool production (48), suggesting $\mathrm{K}^{+}$secretion may also drive water secretion in certain contexts. Our discovery - that $\mathrm{NKCC} 1$ is under transcriptional control by aldosterone to specifically support $\mathrm{K}^{+}$secretion in the distal colon - may be useful in developing mineralocorticoid-based treating diarrheal conditions such as pseudo-obstruction, colitis or renal failure.

\subsection{2 - Remaining questions and future directions.}

What transport pathway mediates aldosterone-induced $\mathrm{K}^{+}$secretion in the proximal colon? 
We and others have demonstrated a major difference between aldosterone-induced $\mathrm{K}^{+}$ secretion in the proximal versus distal segment of the colon. The distal colon is quite similar to the aldosterone sensitive distal nephron (ASDN) of the kidney in that BK channels mediate luminal $\mathrm{K}^{+}$secretion as a means of voiding the body of excess $\mathrm{K}^{+}$. In the proximal colon, it is clear from our own studies, as well as from work by Sandle's group (49) that apical membrane BK channels or IK channels are not up-regulated by aldosterone, despite the obvious increase in luminallydirected $\mathrm{K}^{+}$flux measured by ${ }^{86} \mathrm{Rb}$ tracer experiments (Chapter 5, Table 1; 40,50). This enhanced secretion is also apparently insensitive to $\mathrm{Ba}^{2+}$, leaving two possible explanations: aldosteroneinduced $\mathrm{K}^{+}$secretion in the proximal colon is 1) mediated by a yet undiscovered, $\mathrm{Ba}^{2+}$ insensitive $\mathrm{K}^{+}$channel, or 2) luminal $\mathrm{K}^{+}$secretion is not mediated by a channel at all, but rather through some other mechanism.

As mentioned above, a recent report in mice showed that goblet cells are the principal locus of $\mathrm{K}^{+}$secretion in the colon (41). During degranulation, a large volume of intracellular content (high in $\mathrm{K}^{+}$) is released to the extracellular environment - in this case, toward the lumen. This mechanism may explain the apparent increase in $\mathrm{K}^{+}$secretion observed in the proximal colon of hyperaldosteronaemic animals. Some preliminary evidence supports this possibility, as a protein called CLCA1, a putative goblet cell marker, is significantly increased in hyperaldosteronaemic animals, both in measured protein abundance and number of cells apparently expressing CLCA1 (Appendix Fig. 1). Thus, goblet cell number may increase as a result of elevated aldosterone.

To determine whether goblet cell degranulation contributes to luminal $\mathrm{K}^{+}$secretion, ${ }^{86} \mathrm{Rb}$ flux experiments should be performed under voltage clamped conditions in the presence and absence of an inhibitor of exocytosis. Unfortunately, little information is available surround the key mediators of degranulation in intestinal goblet cells, specifically. A potential candidate 
exocytosis/degranulation inhibitor is the Botulinum family of toxins - inhibitors of vesicular release that work via proteolytic cleavage of VAMP/SNAP proteins. These proteins are expressed in goblet cells as well (51). Experiments using dietary $\mathrm{Na}^{+}$-depleted mice exhibiting intestinal/goblet cell-specific knockout for key $\mathrm{K}^{+}$channels (i.e., BK or IK) would also shed light on this issue because the use of transgenic animals circumvents any technical problems related to pharmacological studies attributable to drug diffusion etc., which can be problematic in Ussing chamber experiments.

\section{Can inhibition of colonic $\mathrm{K}^{+}$secretion ameliorate diarrheal symptoms?}

Enhanced $\mathrm{Cl}^{-}$secretion and reduced $\mathrm{Na}^{+}$absorption are the archetypal causes of water secretion (20). However, excessive $\mathrm{K}^{+}$secretion is associated with several different diarrheal pathologies, including ulcerative colitis, colonic pseudo-obstruction and enterobacterial infection $(44,45,46)$. Previous studies from our lab demonstrate that $\mathrm{K}^{+}$secretion mediated by the BK channel is augmented in the DSS model of ulcerative colitis and that blockage of BK channel with iberiotoxin can inhibit ${ }^{86} \mathrm{Rb}$ flux and water secretion in vitro (52). We also have supporting evidence that BK channel expression is enhanced in UC patients, and that blockage of BK channels can reduce water secretion in perfused DSS-treated rat colon (Appendix Fig. 2). Whether or not inhibition of BK channels in UC patients can meaningfully reduce stool output is still unknown.

Future studies should be direct at confirming the expression pattern of BK or other $\mathrm{K}^{+}$ channels in patients with $\mathrm{UC}$, as well as determining whether any of the associated subunits are also affected. In addition, potential changes in the expression and/or function of NKCC1 have not been explored in the context of UC or other inflammatory bowel diseases, particularly concerning $\mathrm{K}^{+}$secretion. There is evidence that constitutive $\mathrm{K}^{+}$secretion and/or lack of $\mathrm{K}^{+}$absorption is a 
characteristic of UC (53), indicated by high stool $\mathrm{K}^{+}$content and low trans-epithelial potential (cation retention within the gut lumen. Pharmacological and transgenic approaches should be used to abrogate NKCC1/BK-mediated $\mathrm{K}^{+}$transport in DSS-induced colitis, as colonic epithelialspecific knockouts are now available. If inhibition of this pathway at either point (NKCC1mediated uptake or BK-mediated efflux) has a significant effect on water transport, follow-up in vivo studies using intestinal perfusion experiments may support the manipulation of this pathway as a therapeutic option in the treatment of UC. 


\section{REFERENCES:}

1. Adams SM, Bornemann PH. Ulcerative colitis. Am Fam Physician. 2013;87(10):699-705.

2. Camilleri M. Intestinal secretory mechanisms in irritable bowel syndrome-diarrhea. Clin Gastroenterol Hepatol Off Clin Pract J Am Gastroenterol Assoc. 2015;13(6):1051-1052. doi:10.1016/j.cgh.2014.07.020

3. Furness JB. The enteric nervous system and neurogastroenterology. Nat Rev Gastroenterol Hepatol. 2012;9(5):286-294. doi:10.1038/nrgastro.2012.32

4. Spencer NJ, Hu H. Enteric nervous system: sensory transduction, neural circuits and gastrointestinal motility. Nat Rev Gastroenterol Hepatol. 2020;17(6):338-351. doi:10.1038/s41575-020-0271-2

5. Furness JB, Callaghan BP, Rivera LR, Cho H-J. The Enteric Nervous System and Gastrointestinal Innervation: Integrated Local and Central Control. In: Lyte M, Cryan JF, eds. Microbial Endocrinology: The Microbiota-Gut-Brain Axis in Health and Disease. New York, NY: Springer New York; 2014:39-71. doi:10.1007/978-1-4939-0897-4_3

6. Kato A, Romero MF. Regulation of electroneutral $\mathrm{NaCl}$ absorption by the small intestine. Annu Rev Physiol. 2011;73:261-281. doi:10.1146/annurev-physiol-012110-142244

7. Thiagarajah JR, Donowitz M, Verkman AS. Secretory diarrhoea: Mechanisms and emerging therapies. Nat Rev Gastroenterol Hepatol. 2015;12(8):446-457. doi:10.1038/nrgastro.2015.111

8. Cangemi DJ, Lacy BE. Management of irritable bowel syndrome with diarrhea: a review of nonpharmacological and pharmacological interventions. Therap Adv Gastroenterol. 2019;12:1756284819878950. doi:10.1177/1756284819878950

9. Boehlen A, Schwake M, Dost R, et al. The new KCNQ2 activator 4-Chlor-N-(6-chlorpyridin-3-yl)-benzamid displays anticonvulsant potential. Br J Pharmacol. 2013;168(5):1182-1200. doi:10.1111/bph.12065

10. Brown DA, Passmore GM. Neural KCNQ (Kv7) channels. Br J Pharmacol. 2009;156(8):1185-1195. doi:10.1111/j.1476-5381.2009.00111.x

11. Jepps TA, Greenwood IA, Moffatt JD, Sanders KM, Ohya S. Molecular and functional characterization of $\mathrm{Kv} 7 \mathrm{~K}+$ channel in murine gastrointestinal smooth muscles. Am J Physiol Gastrointest Liver Physiol. 2009;297(1):G107-15. doi:10.1152/ajpgi.00057.2009

12. Kurata HT. Chemical regulation of Kv7 channels: Diverse scaffolds, sites, and 
mechanisms of action. $J$ Gen Physiol. 2020;152(8):8-10. doi:10.1085/jgp.202012598

13. Lawson K. Pharmacology and clinical applications of flupirtine: Current and future options. World J Pharmacol. 2019;3192(1):1-13.

14. Field M. Intestinal ion transport and the pathophysiology of diarrhea. J Clin Invest. 2003;111(7):931-943. doi:10.1172/JCI200318326

15. Peiris M, Hockley JR, Reed DE, Smith ESJ, Bulmer DC, Blackshaw LA. Peripheral $\mathrm{K}(\mathrm{V}) 7$ channels regulate visceral sensory function in mouse and human colon. Mol Pain. 2017;13:1744806917709371. doi:10.1177/1744806917709371

16. Shah MM, Migliore M, Valencia I, Cooper EC, Brown DA. Functional significance of axonal Kv7 channels in hippocampal pyramidal neurons. Proc Natl Acad Sci U S A. 2008;105(22):7869-7874. doi:10.1073/pnas.0802805105

17. Gribkoff VK. The therapeutic potential of neuronal KCNQ channel modulators. Expert Opin Ther Targets. 2003;7(6):737-748. doi:10.1517/14728222.7.6.737

18. Lamprecht G, Gaco V, Turner JR, Natour D, Gregor M. Regulation of the intestinal anion exchanger dRA (Downregulated in Adenoma). Ann N Y Acad Sci. 2009;1165:261-266. doi:10.1111/j.1749-6632.2009.04044.x

19. Zachos NC, Tse M, Donowitz M. Molecular physiology of intestinal $\mathrm{Na}+/ \mathrm{H}+$ exchange. Annu Rev Physiol. 2005;67:411-443. doi:10.1146/annurev.physiol.67.031103.153004

20. Kunzelmann K, Mall M. Electrolyte transport in the mammalian colon: mechanisms and implications for disease. Physiol Rev. 2002;82(1):245-289. doi:10.1152/physrev.00026.2001

21. Harish S, Bhuvana K, Bengalorkar GM, Kumar T. Flupirtine: Clinical pharmacology. $J$ Anaesthesiol Clin Pharmacol. 2012;28(2):172-177. doi:10.4103/0970-9185.94833

22. McCartney F, Jannin V, Chevrier S, et al. Labrasol ${ }^{\circledR}$ is an efficacious intestinal permeation enhancer across rat intestine: Ex vivo and in vivo rat studies. $J$ Control Release. 2019;310:115-126. doi:10.1016/j.jconrel.2019.08.008

23. Krugliak P, Hollander D, Ma TY, et al. Mechanisms of polyethylene glycol 400 permeability of perfused rat intestine. Gastroenterology. 1989;97(5):1164-1170. doi:10.1016/0016-5085(89)91686-7

24. Million M, Larauche M. Stress, sex, and the enteric nervous system. Neurogastroenterol Motil Off J Eur Gastrointest Motil Soc. 2016;28(9):1283-1289. doi:10.1111/nmo.12937 
25. Lezak KR, Missig G, Carlezon WAJ. Behavioral methods to study anxiety in rodents. Dialogues Clin Neurosci. 2017;19(2):181-191. doi:10.31887/DCNS.2017.19.2/wcarlezon

26. Diener M, Knobloch SF, Bridges RJ, Keilmann T, Rummel W. Cholinergic-mediated secretion in the rat colon: neuronal and epithelial muscarinic responses. Eur J Pharmacol. 1989;168(2):219-229. doi:10.1016/0014-2999(89)90568-2

27. Neunlist M, Reichelt D, Michel K, Pfannkuche H, Hoppe S, Schemann M. The Enteric Nervous System: Region and Target Specific Projections and Neurochemical Codes. Eur J Morphol. 1999;37(4):233-240. doi:10.1076/ejom.37.4.233.4720

28. Loffet E, Brossard L, Mahe MM. Pluripotent stem cell derived intestinal organoids with an enteric nervous system. Methods Cell Biol. 2020;159:175-199. doi:10.1016/bs.mcb.2020.04.012

29. Workman MJ, Mahe MM, Trisno S, et al. Engineered human pluripotent-stem-cellderived intestinal tissues with a functional enteric nervous system. Nat Med. 2017;23(1):49-59. doi:10.1038/nm.4233

30. Sorensen M V., Matos JE, Praetorius HA, Leipziger J. Colonic potassium handling. Pflugers Arch Eur J Physiol. 2010;459(5):645-656. doi:10.1007/s00424-009-0781-9

31. Rajendran VM, Sandle GI. Colonic Potassium Absorption and Secretion in Health and Disease. Compr Physiol. 2018;8(4):1513-1536. doi:10.1002/cphy.c170030

32. Sørensen M V, Matos JE, Sausbier M, et al. Aldosterone increases KCa1.1 (BK) channelmediated colonic K+ secretion. J Physiol. 2008;586(Pt 17):4251-4264. doi:10.1113/jphysiol.2008.156968

33. Singh SK, O'Hara B, Talukder JR, Rajendran VM. Aldosterone induces active K+ secretion by enhancing mucosal expression of Kcnn4c and Kcnmal channels in rat distal colon. Am J Physiol - Cell Physiol. 2012;302(9):1353-1360. doi:10.1152/ajpcell.00216.2011

34. Bazard P, Ding B, Chittam HK, et al. Aldosterone up-regulates voltage-gated potassium currents and NKCC1 protein membrane fractions. Sci Rep. 2020;10(1):1-14. doi:10.1038/s41598-020-72450-4

35. Lomax B, Mcnicholas M, Lombes M, Sandle I, Mcnicholas M. Aldosterone-induced apical $\mathrm{Na}+$ and $\mathrm{K}+$ conductances are located primarily in surface cells in rat distal colon. 1994;29:G71-G82. 
36. Sørensen M V., Strandsby AB, Larsen CK, Praetorius HA, Leipziger J. The secretory KCa1.1 channel localises to crypts of distal mouse colon: Functional and molecular evidence. Pflugers Arch Eur J Physiol. 2011;462(5):745-752. doi:10.1007/s00424-011$1000-\mathrm{z}$

37. Jiang C, Kawabe H, Rotin D. The ubiquitin ligase Nedd4L regulates the $\mathrm{Na} / \mathrm{K} / 2 \mathrm{Cl}$ cotransporter NKCC1/SLC12A2 in the colon. J Biol Chem. 2017;292(8):3137-3145. doi:10.1074/jbc.M116.770065

38. Alzamora R, Harvey BJ. Direct binding and activation of protein kinase $\mathrm{C}$ isoforms by steroid hormones. Steroids. 2008;73(9-10):885-888. doi:10.1016/j.steroids.2008.01.001

39. Jakab RL, Collaco AM, Ameen NA. Physiological relevance of cell-specific distribution patterns of CFTR, NKCC1, NBCe1, and NHE3 along the crypt-villus axis in the intestine. Am J Physiol - Gastrointest Liver Physiol. 2011;300(1):82-98. doi:10.1152/ajpgi.00245.2010

40. Turnamian SG, Binder HJ. Aldosterone and glucocorticoid receptor-specific agonists regulate ion transport in rat proximal colon. Am J Physiol. 1990;258(3 Pt 1):G492-8. doi:10.1152/ajpgi.1990.258.3.G492

41. Gonzalez-Perez V, Martinez-Espinosa PL, Sala-Rabanal M, et al. Goblet cell LRRC26 regulates BK channel activation and protects against colitis in mice. Proc Natl Acad Sci U $S$ A. 2021;118(3). doi:10.1073/pnas.2019149118

42. Mathialahan T, Maclennan KA, Sandle LN, Verbeke C, Sandle GI. Enhanced large intestinal potassium permeability in end-stage renal disease. J Pathol. 2005;206(1):46-51. doi:10.1002/path.1750

43. Mathialahan T, Sandle GI. Dietary potassium and laxatives as regulators of colonic potassium secretion in end-stage renal disease. Nephrol Dial Transplant. 2003;18(2):341347. doi:10.1093/ndt/18.2.341

44. Van Dinter TG, Fuerst FC, Richardson CT, et al. Stimulated active potassium secretion in a patient with colonic pseudo-obstruction: A new mechanism of secretory diarrhea. Gastroenterology. 2005;129(4):1268-1273. doi:10.1053/j.gastro.2005.07.029

45. Simon M, Duong JP, Mallet V, et al. Over-expression of colonic K+ channels associated with severe potassium secretory diarrhoea after haemorrhagic shock. Nephrol Dial Transplant. 2008;23(10):3350-3352. doi:10.1093/ndt/gfn411 
46. Sandle GI, Perry MD, Mathialahan T, et al. Altered cryptal expression of luminal potassium (BK) channels in ulcerative colitis. J Pathol. 2007;212(1):66-73. doi:10.1002/path.2159

47. Guerrant RL, Chen LC, Rohde JE. Effect of spironolactone on stool electrolyte losses during human cholera. Gut. 1972;13(3):197-200. doi:10.1136/gut.13.3.197

48. Ram P, Goyal A, Lu M, Sloan J, McElhaugh W. Use of Aldosterone Antagonist to Treat Diarrhea and Hypokalemia of Ogilvie's Syndrome. Case Rep Gastrointest Med. 2016;2016:1-3. doi:10.1155/2016/1207240

49. Perry MD, Rajendran VM, MacLennan KA, Sandle GI. Segmental differences in upregulated apical potassium channels in Mammalian colon during potassium adaptation. Am J Physiol - Gastrointest Liver Physiol. 2016;311(5):G785-G793. doi:10.1152/ajpgi.00181.2015

50. Foster S, Hayslett JP, Binder J, Emily S, Hayslew JP, Henry J. Mechanism of active potassium absorption and secretion in the rat colon. 1984.

51. Cornick S, Kumar M, Moreau F, Gaisano H, Chadee K. VAMP8-mediated MUC2 mucin exocytosis from colonic goblet cells maintains innate intestinal homeostasis. Nat Commun. 2019;10(1):4306. doi:10.1038/s41467-019-11811-8

52. Kanthesh BM, Sandle GI, Rajendran VM. Enhanced K+ secretion in dextran sulfateinduced colitis reflects upregulation of large conductance apical $\mathrm{K}+$ channels (BK;Kcnma1). AJP Cell Physiol. 2013;305(9);C972-980. doi:10.1152/ajpcell.00165.2013

53. Sandle GI. Pathogenesis of diarrhea in ulcerative colitis: new views on an old problem. $J$ Clin Gastroenterol. 2005;39(4 Suppl 2):S49-52. doi:00004836-200504002-00004 [pii] 
APPENDIX

A

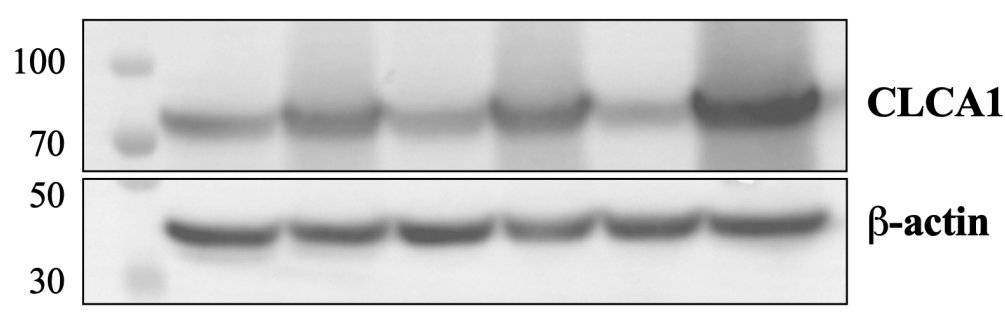

B

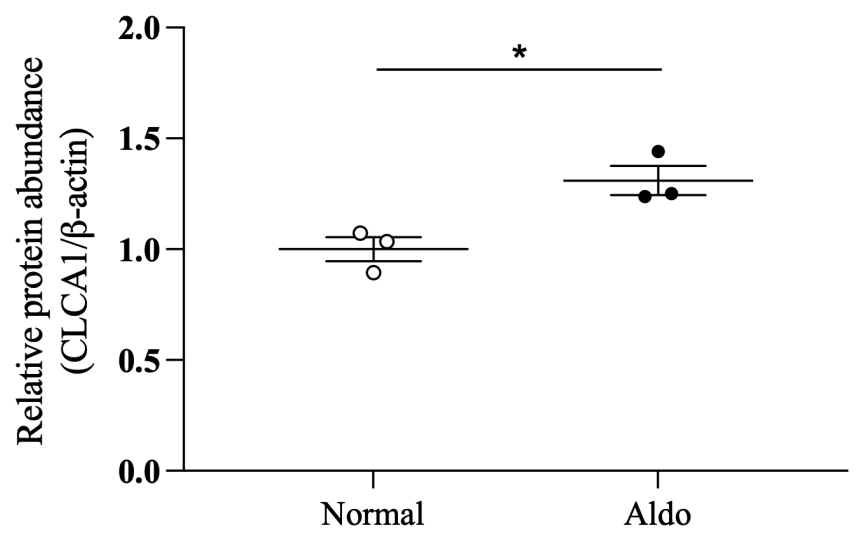

C

Normal

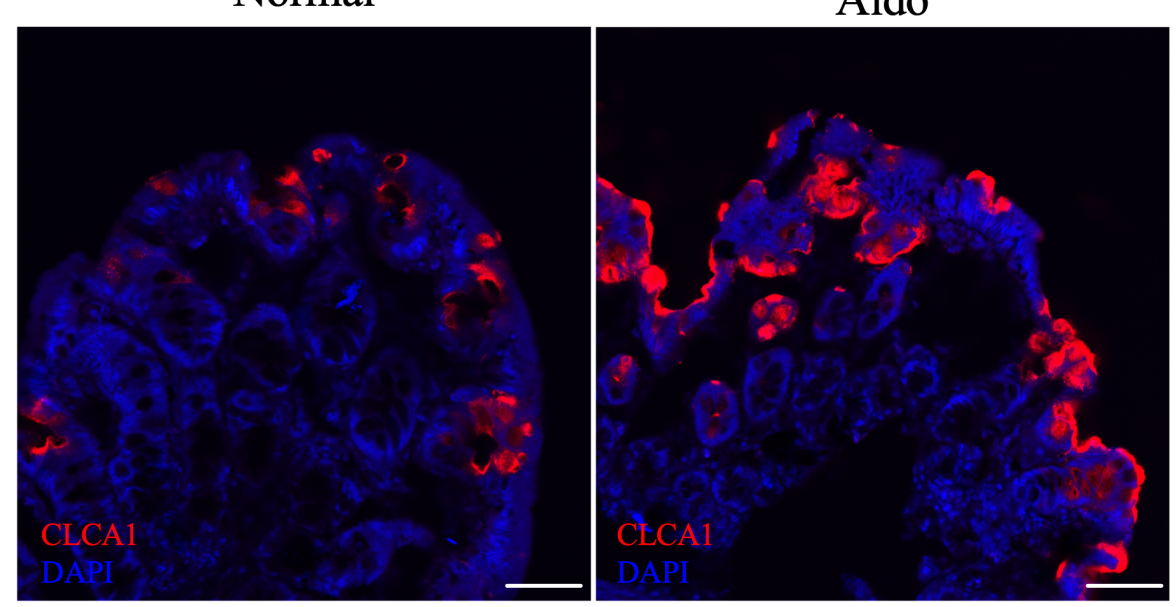


Figure 1 - Goblet cell marker, CLCA1, expression is increased in $\mathrm{Na}^{+}$-depleted ("Aldo") rat proximal colon. $A$ : Representative western blot showing CLCA1 protein from 3 normal and 3 aldo rat proximal homogenates. Standard protein ladder and molecular weights are given in the extreme left lane and to the left of the image, respectively. $B$ : Densitometric quantification of band intensities from blot shown in panel A, normalized to actin as a loading control. Lines and error bars represent mean $\pm \mathrm{SEM} ;{ }^{*} \mathrm{p}<0.05$ as determined by unpaired Student's t-test.

$C$ : Immunofluorescent labelling of CLCA1 in normal and aldo rat proximal colon tissue sections. CLCA1 is shown in red, nuclei are labelled with DAPI and shown in blue. Scale bar $=50 \mu \mathrm{m}$.

A

Human

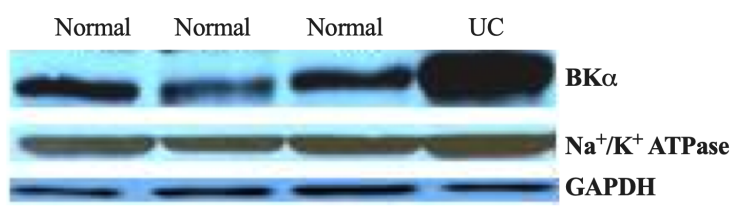

C

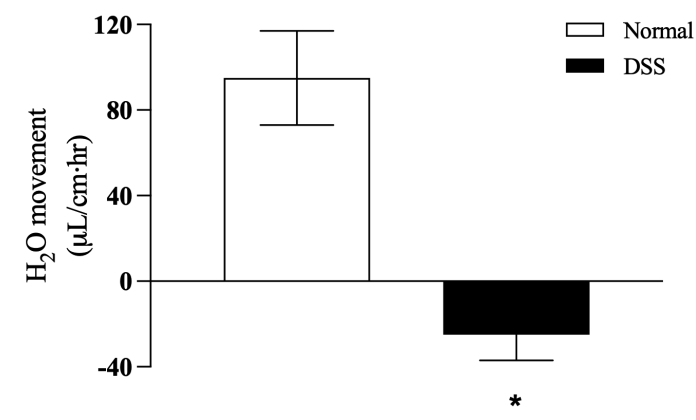

B

Rat

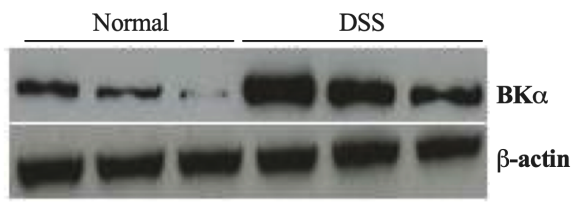

D

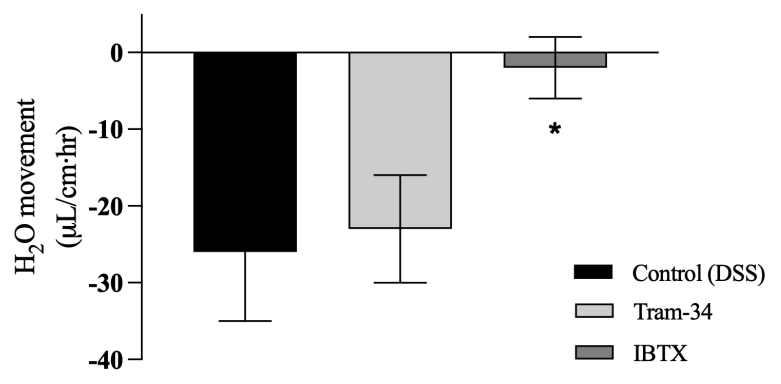

Figure 2 - BK $\alpha$ expression is enhanced in colitis and its inhibition reduces net water secretion. $A$ : Representative western blot showing $\mathrm{BK} \alpha$ protein from 3 normal and $1 \mathrm{UC}$ patient colonic surgical resection homogenates. $\mathrm{Na}^{+} / \mathrm{K}^{+}$ATPase and GAPDH are shown as reference loading controls. $B$ : Representative western blot showing BK $\alpha$ protein from 3 normal and 3 DSS- 
treated rat colon homogenates. $\beta$-actin is shown as a reference loading control. $C$ : Net water movement measured via in vivo intestinal perfusion in normal and DSS-treated rats ( $\mathrm{n}=3$ each). $D$ : Effect of Tram-34 (IK channel inhibitor) and Iberiotoxin (IBTX; BK channel inhibitor) on net water secretion in DSS-treated rat colon measured via in vivo intestinal perfusion. Lines and error bars represent mean $\pm \mathrm{SEM} ; * \mathrm{p}<0.05$ versus control as determined by unpaired Student's t-test $(A)$ or one-way ANOVA with Tukey's post-hoc $(B)$. 